\title{
What does a foot path do
}

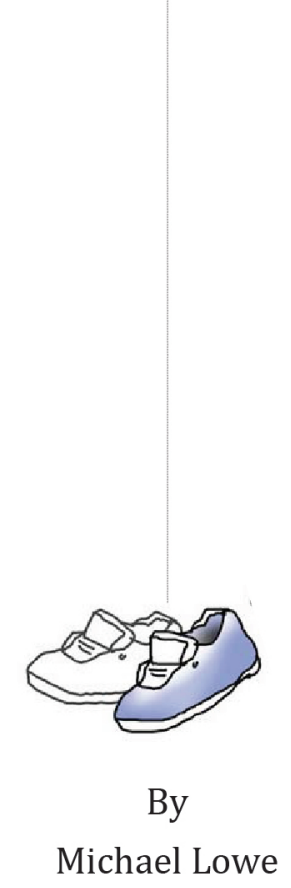

Michael Lowe

A thesis

submitted to the Victoria University of Wellington in fulfilment of the requirements for the degree of Masters of Architecture (Professional)

Victoria University of Wellington

2012 



\section{Abstract}

In New Zealand pedestrian infrastructure is not a robust area of transport planning. As a funding class it receives minimal monetary allocation. This thesis argues design interventions in the pedestrian environment need to be both responsive to the surrounding context and effective in promoting walking patronage. To achieve this goal this thesis argues that the current process used by central authorities and local authorities for researching, designing, and implementing pedestrian infrastructure, needs to change.

As a solution this thesis develops a new methodology for researching, designing and implementing pedestrian infrastructure. The methodology is developed through a series of design experiments using Central Wellington as a site. The methodology uses a multi paradigm framework adopted from landscape perception research, which allows it to successfully alternate between 'top down' expert orientated approaches.

For example, on one hand the methodology uses standardized typological solutions as a cost and time efficient approach to resolving pedestrian barriers to walking; as well as a 'top down' macro scale urban analysis in order to ensure any resources being spent at a micro scale street level are also improving larger urban scale problems in the pedestrian network.

While on the other hand uses a 'bottom up' qualitative approach through a newly developed 'cognitive survey and interview technique', to research from the perspective of the public where pedestrian accessibility barriers to walking are located in the built environment.

The research is significant as it reveals new insight into the practical application of a standardized typological approach to pedestrian design. In particular it reveals how a new hybrid methodology combining a top down expert' and utilitarian approach, with a 'bottom up qualitative' and contextual approach, makes for efficient employment of design resources when implementing pedestrian infrastructure. 
Table of Contents

\begin{tabular}{lr} 
Chapter 1: & $\mathbf{7}$ \\
\multicolumn{1}{l}{ Introduction } & $\mathbf{7}$ \\
1.01 Research Question? & 7 \\
1.02 Research Aim & 7 \\
1.03 Context of the Research: Pedestrian Infrastructure Funding in \\
New Zealand & 8 \\
1.04 Why is a Decline in Walking Patronage Significant? & 8 \\
1.05 Problem Identification: Walking in Wellington New Zealand 9 \\
1.06 Problem Statement? & 10 \\
1.07 Thesis Overview & 10
\end{tabular}

Chapter 2:

Literature review: The behavioural decision making process and designing for pedestrian accessibility in Central Wellington 13

2.1 The Behavioural Decision Making Process:

2.1.1. Environmental Psychology

2.1.2. Environmental Geography

Operational perception

Responsive perception

Inferential perception

Summary
2.2. How Changes in the Built Environment Affect Travel

Behaviour: A Qualitative Understanding of Accessibility

2.2.1 Accessibility and Travel behaviour

$$
\text { Accessibility, }
$$

2.2.2 Measuring Accessibility: The Absence of a Qualitative Approach

2.3 Designing for Pedestrian Accessibility:

2.3.1. Review of Contemporary Walking Behaviour Theory 28

2.3.2. Combining the Three Walking Models

2.4 Current Pedestrian Design Practice in Central Wellington. 35

2.4.1 (Part 1) Pedestrian Design in Wellington: A Lack of

Understanding of the Relationship Between Pedestrian

Accessibility and the Built Environment.

2.4.2 (Part 2) Criticisms of Pedestrian Design Process in

Wellington

2.5. Forming a New Pedestrian Design Methodology 43

2.5.1. Perception Research

43

2.5.2 An Environmental Perception Framework

44

Summary

47

Chapter 3:

49

Methodology

The Methodology 
Chapter 4:

Understanding Site and Pedestrian Accessibility in the Built Environment

4.1 Stage 1: 'Top down' Typological Study

4.1.1 The Experiment Method

4.1.2 Findings

4.2 Stage 2: 'Bottom up' Cognitive Survey Pilot test

4.2.1 The Cognitive Map and Cognitive Mapping

4.2.2 The Experiment Method

4.2.3 Findings From the Cognitive Survey Pilot Test

Conclusions

4.3 Design Test 1: A New Method of Site Analysis Using Typological Pattern Maps \& Qualitative Perception

4.3.1 Method

4.3.2 Findings

4.3.3 Further Direction for the Typological and Cognitive

Mapping Methodology of Site Research

Summary

4.4 Further Direction: The 'New Pedestrian Design Strategy'.

Chapter 5:

81

Pedestrian infrastructural solutions

5.1 Design Experiment 2: 'Creating Typological Solutions' 82

5.1.1 Method

83
5.1.2 Findings

5.1.3 Further direction: The Untapped Potential of Place

5.2 Conclusions of the Typology So Far

106

5.2.1 Further Direction

107

5.3 Design Experiment 3: 'Place-making'

109

5.3.1 Method

109

5.3.2 The design

5.3.3 Findings

5.3.4 Further direction

5.4 Design Experiment 4: 'Rethinking the Larger Pedestrian Network'

5.4.1 Method

'Bottom up' Qualitative Analysis: Survey Results

'Top down' expert analysis

Forming a response to the site analysis

136

5.4.2 Results

1. The Cognitive Survey Process

2. Two Emerging Design Themes in the 'New Pedestrian Design Strategy' 


\section{Chapter 6:}

Discussion: The 'New Pedestrian Design Strategy' and Contributions to Contemporary Theory and Practice

(Part 1) 6.1 Improving Pedestrian Design Practice in Central Wellington:

The Problems with Wellington's Current Pedestrian Design Process:

The 'New Pedestrian Design Strategy': How it can Improve the Problems With Pedestrian Design Practice in Central Wellington.

(Part 2) 6.2 Typologies and the Architecture and Urban Design Professions; and Other Contributions to Contemporary Practice

\section{Conclusion}

182

Bibliography

List of Figures

Appendix

Appendix (A): Typological study

(Access typologies)

(Hybrid typologies)

(Land use typologies)

Appendix (B): Cognitive survey pilot test

Appendix (C): Cognitive survey 1 questionnaire
Appendix (D): Wellington road hierarchy (obtained from

Wellington City Council 2012)

Appendix (E): Table review of pedestrian design practise in Central Wellington

Appendix (F): Cognitive survey 2 questioner (front and back) 230

Appendix (G): Pedestrian count data (obtained from Wellington City Council 2008) 


\subsection{Research Question?}

In New Zealand very little funding is allocated for spending on pedestrian infrastructure. Through combining a top down typological process with a qualitative bottom up design process, this thesis explores how improvements to the pedestrian network can be designed to achieve more than simply increased walking accessibility. Instead, can improving the pedestrian network also be a way to create new and improved public spaces in the built environment? And how can this be achieved considering the limited funding economic environment in New Zealand?

\section{Chapter 1:}

\section{Introduction}

\subsection{Research Aim}

To improve the current processes that Wellington City Council and Central Government use to design pedestrian infrastructure in Central Wellington by:

- Improving authorities' understanding of how pedestrian accessibility is affected by the design of built environment.

- Demonstrating that new interventions to the pedestrian network, which respond to their surrounding context, create opportunities for place-making in the built environment. Further more by capitalising on these opportunities the quality of public spaces in the urban environment can be improved. 


\subsection{Context of the Research: Pedestrian Infrastructure Funding}

\section{in New Zealand}

When Central Government transport spending is compared to transport mode patronage in New Zealand, it is apparent pedestrian infrastructure in New Zealand is a neglected transport mode. In terms of funding, spending on walking and cycling infrastructure in New Zealand has declined over the last three years, as indicated in Figure 1. In addition to this, according to the New Zealand Transport Agency (NZTA), in New Zealand between years 2009-2012 walking and cycling together received just $\mathbf{\$ 5 1}$ Million of the total $\mathbf{\$ 8 7 1 0}$ Million funds being allocated for land transport spending (25-27). This in comparison to the largest spend, New

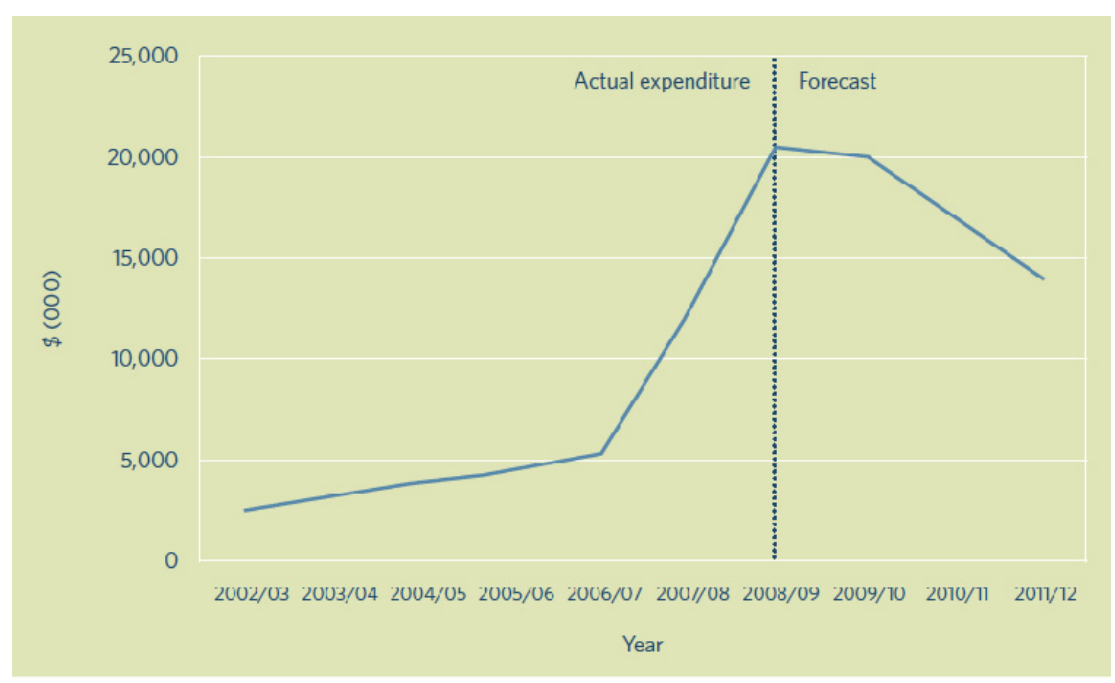

Figure 1: NLTF spending on walking and cycling since 2002 (NZTA 49) and Improved State Highways that received a whopping $\$ \mathbf{3 0 7 0}$ Million (25-27), the outlook for walking is not promising.

$$
\text { Walking and Cycling = \% } 0.59 \text { (total funds } \$ \text { ) }
$$

New and improved state highways = \% 35.25 (total funds \$)

This high roading expenditure has been described by Mees and Dodson as a national bias towards roading infrastructure in New Zealand (1), and is having a negative effect on alternative transport modes such as walking. This is evident in research done by Statistics New Zealand where between the years 1996 - 2006, walking patronage in New Zealand declined in parallel with an increase in the use of the private car as the main means of travel (2).

\subsection{Why is a Decline in Walking Patronage Significant?}

In New Zealand a decline in walking patronage can be associated with an increase in the use of the private automobile. This is significant in a number of ways:

Culturally, if we look to Auckland as an example city, the dominant use of the private car has facilitated urban sprawl, and subsequently an elevated reliance on the road network (Bean et al 2837). Also, high car reliance has 
caused a reduction in the number of child pedestrians present in the street. For example, the literature argues vehicle dependence is causing parents to become 'child chauffeurs' between activities (Bean et al 2837), (Keall 52-53). The subsequent social effect of this is a decline in interaction and public engagement in the community (Litman 11).

Environmentally, the benefit of having more people commute by walking is less reliance on vehicles. This results in less carbon vehicle emissions, and also less development of roadway infrastructure, which both contribute to the global green house effect and rise in respiratory health problems (Duany et al 234), (Hillman 36).

Economically, it is difficult to quantify the benefits of walking into a cost figure (Litman, Alexander Todd 5). However, some of the recognised benefits are:

- On a person to person basis walking commuters have less car dependency meaning less petrol, less running costs, and therefore lower overall transport costs (Litman, Alexander Todd 8).

- Also, less vehicle reliance means cost savings through "less parking facilities, traffic congestion, crash risk, and environmental damages", according to Murphy and Delucchi (Litman, Alexander Todd 9).

\subsection{Problem Identification: Walking in Wellington New Zealand}

Despite the decline in walking patronage nationally, when you look at walking patronage in Wellington, shown in Figure 2, there has in fact been a slight increase between years 2006-2009. Interestingly also, the figure shows one problematic statistic, which is the significant decline in walking trips that occur when the distance a person has to commute increases from 'less than $1 \mathrm{~km}$ ' to 'between 1 to $2 \mathrm{~km}$ '.

\begin{tabular}{|l|ccc|ccc|ccc|}
\hline \multirow{2}{*}{ MODE OF TRAVEL } & \multicolumn{3}{|c|}{ Total } & \multicolumn{3}{c|}{ Trips up } & \multicolumn{3}{c|}{ Trips between } \\
& short trips & \multicolumn{3}{c|}{$1 \mathrm{~km}$} & \multicolumn{3}{c|}{$1 \mathrm{kms}$} \\
& 2004 & 2006 & 2009 & 2004 & 2006 & 2009 & 2004 & 2006 & 2009 \\
\hline Walk & $\%$ & $\%$ & $\%$ & $\%$ & $\%$ & $\%$ & $\%$ & $\%$ & $\%$ \\
Cycle & 37 & 39 & 54 & 73 & 72 & 75 & 16 & 24 & 43 \\
Car or private vehicle & 2 & 3 & 4 & 1 & 2 & 3 & 3 & 3 & 4 \\
Bus or train & 59 & 55 & 33 & 25 & 24 & 20 & 78 & 69 & 40 \\
Other & 2 & 3 & 7 & 1 & 1 & 1 & 3 & 4 & 9 \\
\hline TOTAL TRIPS & $\mathrm{x}$ & $\mathrm{x}$ & 2 & - & 1 & 1 & $\mathrm{x}$ & $\mathrm{x}$ & 4 \\
\hline
\end{tabular}

Figure 2: (GWRC Short trip active mode research 12)

On the map of Central Wellington Figure 3, these 1-2km journey distances are likely to occur anwhere between the $\mathrm{CBD}$ and the residential catchments that are located within a 15-25 min walking proximity of the CBD. 


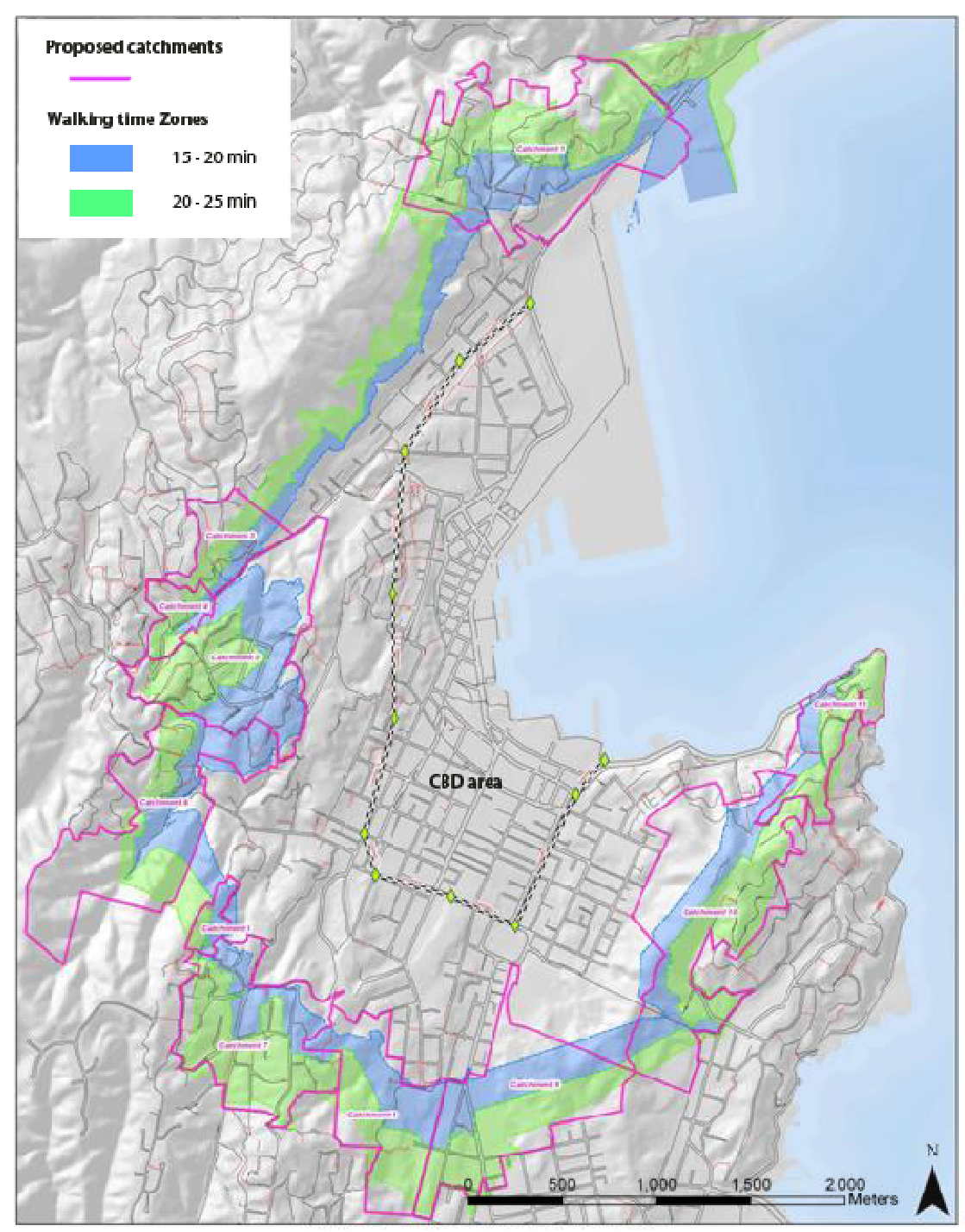

Figure 3: Pedestrian catchments (WCC Walking Times Using Footpath Network)

\subsection{Problem Statement?}

- Why do a significant proportion of people choose not to walk distances between 1-2 km in Central Wellington?

- Is the design of the built environment affecting their travel behavioural decision? And why is this hapenning?

\subsection{Thesis Overview}

\section{Chapter 2 Literature review}

The literature draws from three important research fields: Environmental psychology, Environmental geography, and Urban design. In the chapter four theoretical areas of research will be discussed. These are key to understanding the problem statement in point 1.06.

1. The mental processes that cause behavioural decision making

2. How changes in the built environment affect accessibility and therefore travel behaviour; and as a result why it is important to understanding qualitatively how the design of the built environment affects accessibility.

3. How to improve pedestrian travel behaviour through designing for pedestrian accessibility

4. A proposal for a new framework for perception research: Forming a better pedestrian design methodology. 
Lastly, the chapter will also critique the current pedestrian design practice in Central Wellington.

\section{Chapter 3 Thesis Research Methodology}

In response to the literature review Chapter 3 introduces a 'Hypothesis Design Strategy' as the first attempt to improve the current process for researching, designing, and implementing pedestrian infrastructure in Central Wellington.

Following this, the chapter outlines the methodology used to carry out the design research experiments in this thesis. The purpose of each design experiment is outlined below in Chapters 4 and 5 .

\section{Chapter 4 (Design Experiment 1)}

Chapter 4 begins by testing how successful the initial 'Hypothesis Pedestrian Design Strategy' is when applied in practice. Throughout the experiment as the limitations of this strategy are highlighted, subsequently a new strategy for researching, designing and, implementing, pedestrian infrastructure in Central Wellington begins to develop.

Chapter 5 (Design Experiment 2, 3, \&4)

Chapter 5 has two objectives:
- Firstly to explore the most effective way of researching and understanding what characteristics of a site within the built environment will affect pedestrian accessibility. In order to do this, two techniques from the 'Hypothesis Pedestrian Design Methodology' are trialled as a way of researching pedestrian accessibility barriers within a site. These techniques are a top down' typological study of pedestrian accessibility, and a 'bottom up' cognitive survey of pedestrian accessibility.

- Secondly, to explore how to design cost effective pedestrian infrastructural solutions that are responsive to their context. The chapter initially experiments with using 'standardised typological solutions' as a way for resolving pedestrian barriers in the built environment. However, the limitations of this approach are uncovered, and subsequently in response two further design experiments are carried out using

- A customised design approach, as a way of being responsive to context.

- Macro scale analysis, to ensure all changes made on a micro scale are contributing to the broader urban issues.

In conclusion the chapter suggest a new strategy for implementing pedestrian infrastructure in Central Wellington. 
Chapter 6: Discussion

The chapter discusses in two parts the contributions of this thesis to contemporary practice:

1. Why using a combined 'Top down' and 'Bottom up' approach to researching, designing, and implementing pedestrian

infrastructure can improve pedestrian design practice in Central Wellington.

2. How typologies can be applied to the professions of architecture and urban design. 
The background chapter highlighted a theoretical problem that improving walking patronage in Central Wellington is a behavioural issue. This chapter is divided into five key theoretical areas in order to understand the following four questions:

- What are the metal processes that cause behavioural decision making?

- How do changes in the built environment affect travel behaviour?

- How can pedestrian travel behaviour be altered through the design of the built environment?

\section{Chapter 2:}

\section{Literature review:}

The behavioural decision making process and designing for pedestrian accessibility in Central Wellington
- What is the state of current pedestrian design practice in Central Wellington, and how could it be improved?

The five key theoretical areas are:

2.1 The Behavioural Decision Making Process.

This section gives the reader an insight into the travel behavioural decision making process. It reviews the literature on travel behaviour theory and discusses the three key terms perception, cognition, and accessibility. These terms are taken from the fields of psychology, environmental geography, and urban design respectively, and are important in understanding the travel behavioural decision making process. 


\subsection{How Changes in the Built Environment Affect Travel}

Behaviour: A Qualitative Understanding of Accessibility.

Section two looks at the spatial characteristics of the built environment that affect pedestrian travel behaviour. In particular how the accessibility of the built environment affects travel behaviour. This section also highlights from the travel behavioural literature that the current quantitative research, which examines how the design of the built environment affects pedestrian accessibility, is limited in explanatory power. Therefore more qualitative research needs to be carried out in order to understand this relationship. For example quantitative research cannot be used to question pedestrians on what parts of the built environment they consider to be accessibility barriers. Quantitative research is therefore unable to explain why people make behavioural decisions in response to the built environment.

\subsection{Improving Pedestrian Travel Behaviour Through}

Designing For Pedestrian Accessibility.

This section looks at the theory on designing for walkability, in particular three walkability models by Southworth, Alfonzo, and Metah, which explain how the built environment affects walkability. These models are then used to formulate a spatial framework for assessing pedestrian accessibility in the built environment.

\subsection{Pedestrian Accessibility in Current Pedestrian Design}

Practice in Central Wellington.

This section looks at the current pedestrian design practice for researching, designing, and implementing pedestrian infrastructure in Central Wellington. The section highlights two points:

- The limited way pedestrian accessibility is understood within the two key documents used to design, research, and implement, pedestrian design infrastructure in Central Wellington.

- Secondly, the problems with the current pedestrian design process regarding the use of 'top down' and 'bottom up' approaches to design.

\subsection{A New Framework for Perception Research: Forming A}

\section{Better Pedestrian Design Methodology.}

This final section reviews a useful framework, developed by Taylor, Zube and Sell, for researching environmental behavioural problems, in such as increasing walking patronage in Central Wellington. The framework is useful because it ensures there is a balance between 'top down' and 'bottom up' approaches to the pedestrian design process. In subsequent chapters this framework is used to develop the 'Hypothesis Pedestrian Design Methodology', which through a series of design experiments is 
tested and the findings used to develop a new methodology for designing pedestrian infrastructure in Central Wellington.

The five key theoretical areas will now be discussed:

\subsection{The Behavioural Decision Making}

\section{Process:}

The background chapter highlighted that getting people to walk more in Central Wellington was a behavioural problem. This chapter begins by investigating behavioural theory, to find out what causes people to make behavioural decisions. Behavioural theory argues that a strong relationship exists between the built environment and the psychology of human behaviour (Kaplan 64), (Gold 19), (Handy 196). In order to understand why people make behavioural decisions, the literature from following two key research fields must be examined (Tolman 27):

- Environmental psychology, which focuses on understanding psychological processes.

- Environmental Geography, which focuses on the relationship between the physical environment and human behaviour.

\subsubsection{Environmental Psychology}

The field of Behavioural Analysis within Environmental Psychology is important to understanding why people's behaviour is affected by the built environment. Behavioural Analysis is: 
"the science that studies environmental events that change

behaviour (Miller 5)",

Note 'environmental events' are those events that occur in a person's surroundings (Miller 5). Behavioural Analysis is important to this thesis because the field investigates the principles that alter human behavioural 'problems' (Miller 159).

\section{The Behavioural Principle}

Eminent behaviourist B F Skinner believed the essential principle of behaviour was:

\section{"Organisms tend to repeat responses that lead to positive}

outcomes, and they tend not to repeat responses that lead

to neutral or negative outcomes (Weiten 8)."

For example, when I commute from home to university I have a choice whether to walk or drive. Ten times out of ten I will walk although the trip takes longer to walk than drive. But why is this? As a 'poor' student I enjoy using the $\$ 10$ per day, which would be otherwise spent on parking, to buy a nice lunch in town. Because I consider buying lunch a positive outcome, I walk more and seldom take the car. This behavioural principle is important to this thesis because instigated further research investigating how human behaviour can be intentionally altered. As a result of this research Skinner developed the term Operant Conditioning and within it the two important concepts of Positive and Negative Reinforcement, which this thesis argues are significant towards altering people's travel behaviour through the design of the built environment.

\section{Operant Conditioning: Positive and Negative Reinforcement}

Operant conditioning can be defined as a:

"form of learning in which responses come to be controlled

by their consequence (Weiten 224)."

The principle of operant conditioning is applied through Skinner's concept of Reinforcement (Weiten 225), whereby a 'reinforcer' is used to increase how often a behaviour occurs (Miller 159). A 'reinforcer' is an event that occurs after a behavioural action, and increases the rate of the behavioural action (Miller 159). This is illustrated in Figure 4:

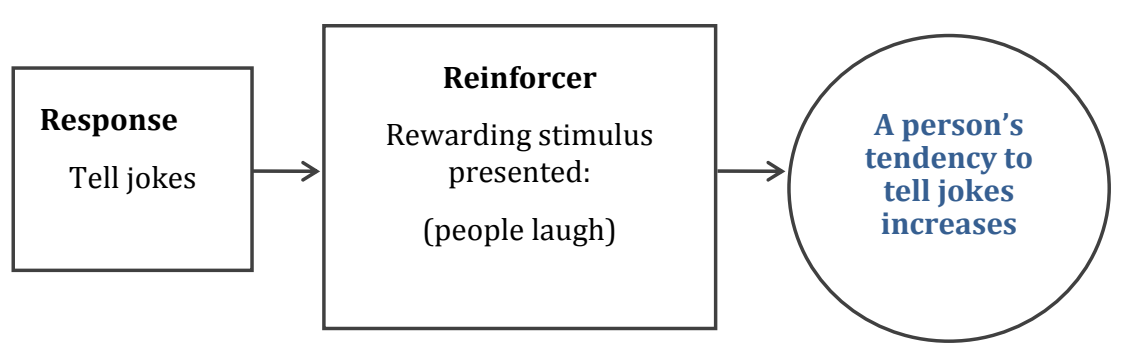

Figure 4: Example of a Reinforcer (Weiten 226) 
Reinforcers occur in both positive and negative categories:

\section{- Positive Reinforcement}

Occurs when a behavioural response is strengthened due to the presence of a pleasing stimulus (Nevid 176),

\section{(Weiten 223).}

\section{- $\quad$ Negative Reinforcement}

Occurs when a behavioural response is strengthened due to the removal of a disliked stimulus (Nevid 176),

(Weiten 223). In terms of the design of the built environment, a 'stimulus' can be considered anything within the built environment that causes a behavioural response.

These two behavioural principles from psychology are important to understanding how the design of the built environment affects travel behaviour. For example, because Central Wellington's built environment is constantly changing, as a result so do pedestrians' experiences of the built environment. Therefore, considering throughout this process the two behavioural principles are actively engaged, changes in the built environment are affecting people's travel behaviour for better or worse.
The following section will now look at the behavioural decision making process from the perspective of the Environmental Geographer. The research field is important because it can explain how the mind processes change in the built environment that subsequently affects human travel behaviour.

\subsubsection{Environmental Geography}

Within the field of Environmental Geography in order to understand why individuals make travel decisions such as the decision to walk, it is important to examine the mental processes that mediate between the environment and a person's travel behaviour (Alfonzo 809). These mental processes are perception and cognition. Gold states that perception and cognition are the most influential elements in,

\section{"Individual-to-environment transactions...", as they are, \\ “...the internal mental processes by which individuals sense, perceive, interpret, and make decisions about their environment (19)."}

These mental processes of perception and cognition are important because they are the connection between the spatial environment and people's travel behaviour (Hannes, Janssens and Wets 76). Therefore, understanding individuals' perception and cognition of the pedestrian environment is crucial towards understanding their travel choice decision 
making. The significance of each of these processes will now be covered in more detail.

\section{Perception}

Perception is defined as the "immediate apprehension of information about the environment" through the senses, which arises when an object is present (Golledge 190). It is a process that involves recognizing and understanding new environments by "capitalizing" on information learnt from past experiences (Kaplan 67). Theory developed by Kevin Lynch, Jan Gehl, and Donald Appleyard, suggests that the perceived nature of the built pedestrian environment may influence an individual's decision to walk (Handy 186). In this sense perception is significant because the design of pedestrian infrastructure in the built environment therefore will affect how pedestrians initially process the environment, and subsequently whether they have a positive or negative walking experience. For example on my walking journey to University, I constantly have an unpleasant experience along a certain busy road because I have to walk dangerously along a narrow footpath with no buffering between fast passing cars and myself.

This section will discuss the theory explaining how perception is used by individuals to process information in the environment. Within perception there are two types of spatial information that influence people's decision making in the pedestrian environment (Appleyard 112), (Golledge 190), (Gold 50):

- Primary sensory information, which is information received directly from the environment by means of sensory experience. For example, as you walk through a busy car park you feel vulnerable and uncomfortable around the manoeuvring vehicles, causing you to avoid walking through that car park.

- Secondary information on the other hand is information received through communication with people and what is seen in the media. For example, you might believe a certain part of your route home is dangerous because you have heard so through conversation with friends, although you may never have experienced this danger in person.

Appleyard argues that within these two categories of spatial information there are also three more ways that individuals process sensory information. These three further categories can affect a person's travel behaviour and have become cemented in Environmental Geography theory (Carter 353):

- Operational perception,

- Responsive perception,

- Inferential perception (Appleyard 111), 


\section{Operational perception (choosing)}

Appleyard defines operational perception as the process, for a commuting individual carrying out tasks in the built environment, of choosing "particular aspects of the environment for the purpose of carrying out these tasks (109)". During this process the chosen aspects of the environment are perceived and remembered as operational "reference points" to the commuting individual (Carter 340). Note these operational reference points have a functional purpose. Therefore, when a person is commuting,

"An operationally complex environment, which can be used in many ways, may be much more satisfying than one that only creates complex images (Appleyard 110)".

For example, when a commuting individual perceives and uses objects in the environment; e.g. when a pedestrian uses a laneway as a means of a short cut to their destination; the perceptual information of the laneway experienced through their journey will be much stronger than that of a pedestrian who doesn't use the laneway, and only perceives it in passing. This is because in the latter the laneway gives no operational contribution to the pedestrian's activity, and thus is of less significance in their travel behaviour decision making process.

In this sense functional aspects of the built environment that affect a pedestrian's journey feature more strongly in the perception process. The aspects of the built environment that interfere with the functionality of a pedestrian's environment with regards to walking, have a strong negative influence on the perception process and thus a pedestrian's travel behaviour.

\section{Responsive perception (reacting to environment)}

In contrast to operational perception, which is an active perception process for a commuter, is responsive perception, which is a passive process. Passive because responsive perception takes place unintentionally while a commuter is navigating in the environment. Appleyard describes responsive perception as being,

"Distinctive elements intrude on the operational search patterns of the traveller (110)."

These elements are what Lynch theorised as the "imageable" parts of the environment that contribute to the process of navigation and way finding (Appleyard 110). They come in any form such as landscape, building, including visual mediums such as signage or advertising (Carter 341), and can be identified through any sensory experience, not only sight (Lynch $10)$.

\section{Inferential perception (deciding)}

Appleyard defines inferential perception as, 
"The fitting of information into categories, predicting

probabilities, forming and testing hypotheses...A kind of

process that that leads to conclusion based on reason

(110)."

In a new situation, an individual's actions are concluded based on information learned from past experience (Carter 341). Note that the multidisciplinary use of the terms perception and cognition terms in the fields of Geography, Psychology, and Urban Design, has confusingly caused inferential perception to merge similarly to the definition of cognition (which will be discussed in the next section). However according to Gold, these differences in terminology are not significant, but only are research "heuristic devices (20)". Therefore the principle of inferential perception can be considered the same as the principle of cognition, which will be explained after the following summary.

\section{In summary of the theory on perception:}

1. Firstly, individuals receive spatial information in the environment through the mental process of perception, and this affects how individuals respond to the built environment.

2. Secondly, as a result of the perception process, the travel decisions individuals make through perception appear to be two sided. On one hand informed by the mind of the individual through the perception of 'primary sensory information':
- Operational perception, choosing and using elements in the environment that enable a certain task to be performed; Along with inferential perception, the cognitive process of making decisions based on previous experience.

However, on the other hand travel decisions are influenced subconsciously by:

- Sensuous distracting objects in the built surroundings through the process of responsive perception (Appleyard 111); as well as 'Secondary information' through mass media and communication with others.

Cognition

Cognition is the mental process by which individuals "code and organise (Golledge 190 Spatial behaviour)", and "store and operate upon (Gold $20)$ ", the information of the built environment gained through perception. Downs explains that cognition functions at a larger environmental scale than perception, and at a level beyond the immediate human sensory field (14). Cognition is not associated with immediate behaviour, but with past experience and understanding what will occur in the future (14). For example an entire environment is never understood at one instant. Instead when people experience the built environment their complete understanding of the environment is formed over multiple perception 
experiences over time, and information gathered from the different mediums of perception.

Cognition is significant to understanding a person's travel behaviour for two reasons:

- Firstly, it influences a person's decision making. It is the mental processing of,

"Perception, imagery, retention and recall, reasoning and problem solving, and the making of judgements and evaluations (Golledge 191)."

- Secondly, an outcome of the cognition process is that a person develops a,

"Mental representation of the environment

(Golledge 191)."

See bottom of Figure 4 page 16. This is significant because people's behaviour is a reaction towards their mental representation of the environment, not the environment itself (Golledge 191)

A person's mental representation of the environment' is significant in terms of uncovering what aspects of the built environment affect their travel decision making. This thesis argues that tapping into people's cognitive processes, and thus their transformed mental image, will reveal why people choose not to walk as a result of the built environment. ${ }^{1}$ In this sense cognition plays an important role in this thesis in terms of understanding travel behavioural decision making because it is the final process that determines how people comprehend and form their opinions of their experiences of walking in the built environment.

\section{Attitudes}

As a final order of address, attitudes will be discussed. Attitudes can be defined as 'learned predispositions' that cause an individual to act in a particular way towards an object (Gold 23), (Downs 14), (Golledge 200). Attitudes are important because they are a hidden variable when trying to understand how the built environment affects people's travel behaviour. This is because a strongly grasped attitude can cause individuals' to "predispose behavioural actions" regardless of the influence of the built environment (Gold 23). Collective research by Snellen, Aloys, and Timmermans has found that "Mobility nature" or attitude of people can affect people's travel behaviour (1207).

This is significant in relation to the design of the pedestrian environment for two reasons:

${ }^{1}$ Note a technique called cognitive mapping can be used to abstract a person's mental representation of the environment. This technique will be introduced in Chapter 4. 
- Firstly, design improvements to the pedestrian environment might not affect a person's travel behaviour if a pedestrian has a strong attitude is towards some aspects of the built environment (Gold 25).

- Secondly, repeated past good or bad experiences of the built environment can contribute toward the formation of an attitude (Downs and Stea 14). Therefore there is the ability for the design of the built environment to contribute to the formation of an attitude, and subsequently, affect people's travel behavioural actions in regards to walking for better or worse.

\section{Summary}

In summary of this review of the behavioural decision making process, the following points can be noted:

- Firstly, people experience the built environment directly through perception. Over multiple experiences their mind then reflects and interprets these experiences through the cognitive process.

- Secondly, an outcome of both the perception and cognitive process is that people develop a mental image or representation of the environment. This is significant towards understanding travel behaviour because people make behavioural decisions based on their mental representation of past experiences of the built environment.
- Thirdly, tapping into people's mental representation of the built environment is therefore a crucial step towards learning how pedestrians perceived the built environment through the mental processes of perception and cognition, and subsequently what aspects of the built environment are affecting their decision to walk. This is significant because learning how pedestrians perceived the built environment enables pedestrian infrastructural design interventions to be targeted to improve people's walking experience and therefore also their travel behaviour.

- Fourthly, the design of the built environment can contribute to the formation of an attitude, which can subsequently affect people's travel behavioural actions in regards to walking for better or worse. 


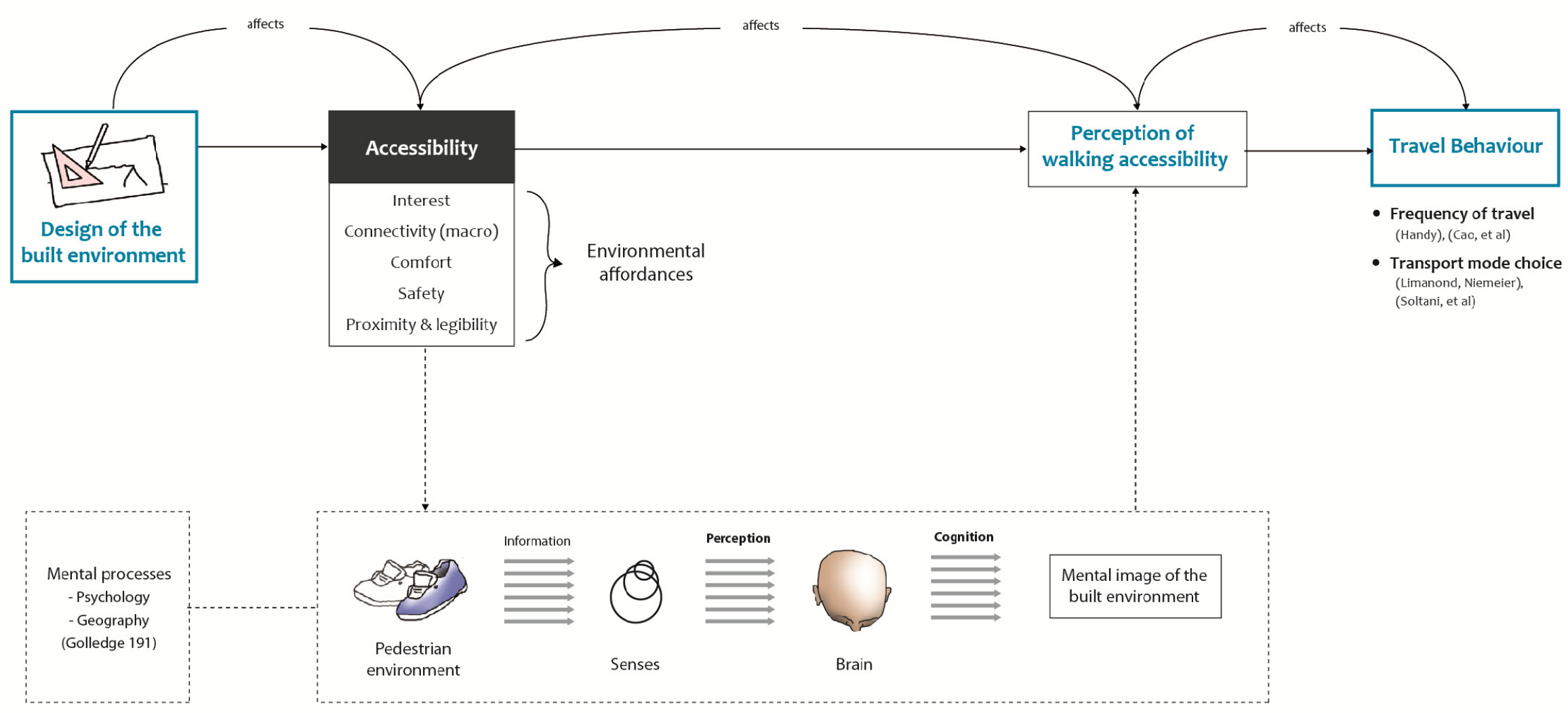

Figure 5: Map summarising how the design of the built environment affects travel behaviour.

Note this discussion so far has given the reader an insight into the behavioural decision making process. By the end of this chapter, the reader should be familiar with Figure 5, which shows in summary the key theory behind why the design of the built environment affects pedestrian accessibility. 


\subsection{How Changes in the Built Environment}

\section{Affect Travel Behaviour: A Qualitative}

Understanding of Accessibility

This section will be discussed in two parts

- Firstly, how accessibility in the built environment affects a person's travel behaviour.

- Secondly, why the limited qualitative understanding of how the design of the built environment affects accessibility is problematic when designing pedestrian infrastructure.

\subsubsection{Accessibility and Travel behaviour}

Currently within the field of Environmental Geography there is limited research identifying the spatial characteristics of the environment that affect commuting (Alfonzo 827), and this has arguably contributed to the debate as to whether the built environment influences people's travel behaviour.

On one side the theory argues the built environment influences people's travel behaviour in two ways (Naess 648), (Thill and Kim 246):

- The frequency of travel: Whether it by private vehicle, walking, or cycling (Handy 196), (Cao and Mokhtarian 554).
- The transport mode choice: Meaning the decision to travel by one mode over another (Limanond and Niemeier 237), (Soltani, Primerano and Allan 185).

The factor common to both of these travel behavioral occurrences is the accessibility of the built environment. Accessibility is defined as,

\section{Accessibility:}

"The ability of a person to be able to access the destination; the distances to a destination; the physical and perceived barriers to walking to a place; and the connectivity between land uses (Mehta 220).”

where 'the ability' to reach a desired destinations refers to time, cost, and effort (Pucher 166).

The relationship between perception cognition and accessibility is important to this thesis in terms of understanding how people's travel behavioural decision making is affected by the design of the built environment. This is because how people perceive accessibility in the built environment has been found to affect travel behavioural decision making (Hannes, Janssens and Wets 96). Research by Alfonzo demonstrates an example of this relationship between accessibility, perception, and cognition. When a person carries out necessary activities - the "compulsory" activities that individuals are required to carry out within the urban environment, such as commuting to work, or waiting for a bus 
(Gehl 365) - the perception of distances and perceived barriers to a destination can affect a person's understanding of how accessible the built environment is (Alfonzo 826-827).

On the other side of the debate there is research claiming only a modest relationship exists between people's travel behaviour and the built environment. However many of these arguments are described as being invalid because the conclusions often come from problematic computer simulation experiments (Naess 649). These simulation experiments are flawed because they require a computer to predict how the environment will affect people's travel behaviour. As a result the quality of the simulation relies on the quality of the researchers inputted assumptions of how they understand the 'built environment vs. travel behaviour' relationship. In terms of this thesis therefore, I argue the travel behavioural theory derived from simulation cannot be used to further understand how the design of the built environment can cause people to walk more. Consequently the first side of the argument in which the theory does so will be focused on in this thesis.

\subsubsection{Measuring Accessibility: The Absence of a}

\section{Qualitative Approach}

The travel behavioural literature argues there is a need for a better understanding of how the built environment affects pedestrian accessibility (Alfonzo 827), (Mondschein, Blumenberg and Taylor 849). Bean writes,

"Traditionally, transport literature has been dominated by quantitative research, with many studies using techniques such as travel surveys or traffic volume data in order to understand travel behaviour (2831)."

This has caused the research to date on accessibility to be focused on understanding the quantifiable measures. Some of these measures include researching whether geographical distance from a destination affects travel frequency, by the likes of Alfonzo; Schlossberg, Greene and Phillips; and Thill and Kim. However, there is still a lack of qualitative understanding underlying why we make behavioural decisions (Hannes, Janssens and Wets 79), (Crane 18). In particular little is known about how people's travel behavioural decision making is affected by certain spatial characteristics of the built environment (Zacharias 4). This lack of qualitative information is a problem because qualitative information is vital towards understanding what parts of the built environment affect pedestrian accessibility (Crane), (Hannes 76), and therefore a person's travel behaviour (Handy 196). I argue this is significant, as authorities 
need to be aware of what in the built environment is causing people to walk less, so the problems can be targeted through new pedestrian infrastructural improvements to the built environment.

Furthermore, Zacharias argues that relying on quantitative information alone is problematic when designing for the pedestrian environment because the design process,

\section{"requires assumptions about how pedestrians will respond}

to characteristics of the environment (3)."

This lack of qualitative research is therefore significant, as qualitative knowledge provides a different kind of information to designers. In particular from the perspective of the user, what the characteristics of the built environmental are that impact negatively on pedestrian accessibility. For example, the following focus group experiment conducted by Bean, Kearns and Collins, explored social mobilities of walking and driving in New Zealand. In the experiment the members of the focus group did not allow their children to walk to school because they felt Auckland's roads and streets were dangerous and unsafe. As a result the parents were chauffeuring their children to school by car (2841). This qualitative information from the focus group is significant in terms of resolving the 'safety' problem through the design of the built environment. By understanding qualitatively what aspects of the built environment were affecting the parents' perceptions of pedestrian accessibility, each aspect could then be resolved effectively through a specific design intervention. For example a strategy could be developed for improving the safety of the pedestrian and vehicle interface along the key routes the children took to school.

In summary of this section the travel behaviour theory has highlighted:

- That accessibility of the built environment affects a person's travel behaviour.

- $\quad$ There is a lack of qualitative research relating to how the design of the built environment affects pedestrian accessibility and therefore travel behaviour.

Therefore in recapping this chapter so far:

- Firstly, perception and cognition are the mental processes by which individuals make decisions about their environment (Gold 20).

- Secondly, accessibility of the built environment, in terms of the physical and psychological barriers to walking, plays an important role in how individuals choose to travel (Hannes 96), (Naess, Accessibility), (Thill, Kim 246).

- Thirdly, in the design of the pedestrian environment it is important to understand qualitatively how individuals perceive accessibility in the built environment. Therefore in combining all these points together; by understanding qualitatively, and designing for, individuals' perceptions of pedestrian accessibility 
in the built environment, this thesis argues the pedestrian

infrastructural improvements can influence people's travel

behaviour and thus the likelihood of them walking more.

Lastly as a final point this chapter has highlighted that a person's travel behaviour is influenced by the built environment as well as their mental attitude. However, it should also be acknowledged that there is one other influence in the form of socio-cultural and economic circumstance (Cao and Mokhtarian 538), (Mondschein, Blumenberg and Taylor 863).

The next section will discuss how to design for pedestrian accessibility, and what spatial characteristics of the built environment are relevant to accessibility. This will begin by examining the theory on walkability, which is key to understanding how the design of the environment affects the pedestrian experience. 


\subsection{Designing for Pedestrian Accessibility:}

Having highlighted that accessibility is important in understanding pedestrian travel behaviour, the next step was to establish a framework for accessibility for three reasons:

- To understand what spatial characteristics of the design of the built environment affect pedestrian accessibility, and therefore pedestrian travel behaviour.

- To provide a useful tool for thoroughly reviewing how accessibility is understood in current pedestrian design practice in Central Wellington, and therefore how well travel behavioural theory is considered in current practice in Wellington for improving walking patronage.

The section will begin with a brief review of contemporary theory on walking behaviour, before using the review to formulate a new framework for assessing pedestrian accessibility.

\section{Theory}

Exploring how the built environment affects walking behaviour is a recent field of literature. In this sense it can be argued that pedestrian design is only recently becoming more important in the design of cities. For example, only in the last decade in America has designing for walking become a major part in the planning of transport infrastructure and cities (Southworth 246). This similarly can be seen in New Zealand. The Pedestrian Planning and Design Guide, the first national publication focused towards improving the pedestrian environment in the design of cities, was only published in 2007 by The New Zealand Transport Agency. Southworth argues that over the course of history urban design and transport planning have separated in their development into two professional fields:

"Urban design focusing on the concrete experiential qualities of the built environment, generally at small to medium scale (246). "

"Transportation planning focusing on more abstract function and efficiency, particularly for the motorist, at the scale of cities and regions (246). “ 
This specialisation has led to problems in the execution of transport planning in cities. For example it is often apparent that transportation planners fail to consider the quality of the environment from a pedestrian perspective, due to a greater focus on what is considered to be the larger issue of vehicle transport (Ramsay 159-171). Factors such as the quality of the environment are among the frequently ignored opportunities for improving 'non-motorised transport' by transport planners (Mobility Management Litman 7). In a New Zealand example this is evident in Auckland. Auckland is known as one of the worst automobile dependant cities in the world (Mees and Dodson, Backtracking Auckland: Bureaucratic rationality and public preferences in transport planning. 1), (Gehl and Newman, in Auckland City of Cars), and is still suffering from half a century of 'roading obsessed' transport planning initiatives (Mees and Dodson, The American Heresy: Half a Century of Transport Planning in Auckland).

Three recent researchers, Alfonzo, Southworth, and Mehta, found that in the areas of transport planning and the design of cities, research on pedestrian behaviour is still incomprehensive. In response, these authors' research has led to two contemporary theoretical walking models being developed, of which each model is made up of the key attributes believed to be essential measures of walkable environments. To begin with the 'theoretical walking models' will be explained. Then following this, in order to establish a theoretical framework for assessing how the design of the built environment affects pedestrian travel behaviour, the models will be rearranged to form a new theoretical framework for pedestrian accessibility.

\section{Walking model 1: Southworth 2005}

Southworth's research was concerned with the way walkability theory focused so heavily on criteria of "utilitarian access (248)" - i.e. the physical distance to a destination. In response, Southworth proposed six new criteria for evaluating walking in the built environment. These were (Southworth 249-254):

- $\quad$ Linkage to other modes, referring to the connectivity the pedestrian path network has between other transport modes such as train or bus.

- $\quad$ Connectivity, referring to the path network's "continuity and absence of significant barriers (249)." For example highways with large traffic volumes dissecting a pedestrian path network.

- $\quad$ Fine grain and varied land use patterns, referring to the distribution of key activities in the city that serve every day needs, as well as how well a person can access these activities by walking reasonable distances. 
- $\quad$ Safety referring to a person's perception of safety such as fear of traffic or crime.

- Quality of path, referring to how user friendly the path is, and the purpose of the path for being either pedestrian oriented versus vehicle oriented. Path quality is also affected by pedestrian amenities such as planting, landscaping, footpath width, signage and utility obstacles on the path etc.

- $\quad$ Path context, referring to the how the path permits interaction with the surrounding context in order to create physical as well as visual interest to the user. Path context is affected by elements such as,

\section{"scale of street space, presence of street trees and} other landscape elements, views, visible activity and transparency, scale, and coherence of built form (254)."

\section{Walking model 2: Alfonzo 2005}

Alfonzo's research, published in the same year as Southworth's, criticised the walkability literature for considered only limited physical characteristics of the built environment that affected pedestrian behaviour (809). Alfonzo argued that this was causing there to be a limited understanding of how the built environment affected walking
(809). In response a theoretical model was developed called 'The Hierarchy of Walking Needs' Figure 6.

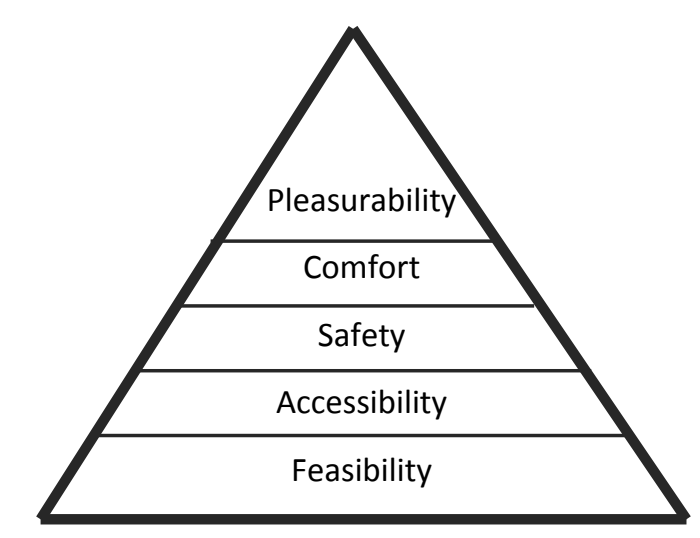

Figure 6: Hierarchy of Walking Needs (Alfonzo 825)

The model works on the basis that places in the built environment offer varying levels of affordance to the categories of the hierarchy. This term affordance, used by Alfonzo, meaning the accepted level

\section{"of properties that are present within an environment that allow for the occurrence of a behaviour (Alfonzo 818)."}

Note Alfonzo's 'walking' model is a derived from a much older Hierarchy of Needs model that was theorised by psychologist Maslow. The key principle of Maslow's theory was that people are only prepared to act upon, or be influenced by, a higher tier in the hierarchy only if the lower tiers or "deficiencies" have been fulfilled (Huitt). 
Expanding of the five levels within the hierarchy: (Alfonzo)

- $\quad$ Feasibility refers to how practical the walking trip is i.e. is it possible to reach the destination under a time deadline by a given mode of transport. For destination trips i.e. not leisure trips, feasibility could contribute to the choice of transportation mode.

- $\quad$ Accessibility refers to the

"quantity, quality, variety, and proximity of

activities present, as well as the connectivity

between uses (Alfonzo 826)"

According to Alfonzo's theory accessibility can also refer to the physically and psychologically understood barriers to walking i.e. a person's perception of distance or safety. Note this is a much more basic definition of accessibility that this thesis chapter gives on $p g$ 25, and to the accessibility framework this chapter is trying to establish.

- $\quad$ Safety refers to whether a person feels safe from the threat of crime. Attributes of the environment such as land use, the absence of eyes 'on the street', lighting conditions, and presence of certain social groups, can all effect a person's understanding of safety.
- Comfort refers to the "ease, convenience, and contentment (828)" a person feels in the environment. Attributes of the built environment include:

- Elements that affect the pedestrian experience of motorized traffic (i.e. traffic calming, speed limits, etc);

- The quality or state of the walkway network, protection from undesirable weather conditions such as street canopies;

- The presence of amenities (seating, public toilets, drinking fountains, etc).

- $\quad$ Pleasurability refers to how appealing the walking scene is. Attributes relating to this could include the presence of architectural features (contemporary and historic), variety in streetscape aesthetic and street activity.

\section{Walking Model 3: Metah 2008}

Developing further on Southworth's and Alfonzo's research, Metah's research critiqued the previous models for failing to consider the following: (Mehta 219)

- The usefulness of activities located along a path or at a destination 
- The influence the path or destination has on social interaction for the user. For example some routes may be used because there are more people around, or have a connection to a place such as the family running the local convenience store.

Metah subsequently extended the Hierarchy of Walking Needs model to include two further needs/categories:

- $\quad$ Usefulness, which is an expansion on Southworth's Fine Grained and Varied Land-use Patterns, embodies the idea of land use intensity and land use diversity as factors influencing the walking proximity of key urban activities i.e. school, supermarkets etc (Southworth 250). This definition of usefulness is similar to Alfonzo's definition of accessibility in that they both involve the principle of proximity to a destination.

- $\quad$ Sense of belonging referring to places encouraging sense of community, which "may often cater to mundane but essential everyday functions...that help in establishing their community's identity (222)." In this sense people may choose to walk through or past places that are of personal value. For example, I often walk down a certain street because I enjoy walking past my old primary school.

\subsubsection{Combining the Three Walking Models}

Southworth, Alfonz, and Mehta's theorised walking models give a comprehensive insight into the literature examining walkability, as well as how pedestrian travel behaviour is affected by the built environment. This thesis argues there are three reasons why it is appropriate to combine all the models into one framework for accessibility. These are:

1. These walking models are the only theory investigating how the built environment affects pedestrian travel behaviour, which makes them important towards understanding pedestrian accessibility.

2. The walking models vary between each author, yet when comparing each to their counterpart, neither model seems entirely robust. This thesis argues the missing points from each model can be resolved by bringing them together under one framework.

3. The overlapping definitions between the models are confusing in the sense that each model has different names and overlapping definitions for the same terms. Thus bringing the models together would remove this confusion of repeated terms and definitions.

Therefore considering the definition of accessibility used in this thesis, below,

"the ability of a person to be able to access the destination; the distances to a destination; the physical and perceived 
barriers to walking to a place; and the connectivity

between land uses (Mehta 220).”

and after examining the relationships between the sub categories of the three walking models, the following accessibility framework was created (Figure 7). The framework is divided into sub categories, and all together can explain why the built environment affects pedestrian accessibility, and therefore pedestrian travel behaviour.

\section{A couple of things should be noted about this framework:}

- Firstly the category of connectivity is of particular relevance to the built environment at the macro urban scale in terms of land use patterns, density, and urban form. The other categories however, have more relevance at the micro scale at the level of the footpath and its immediate surrounding context.

- Secondly, it must be acknowledged that there is a subjective element in devising the subcategories of the framework. Therefore, although elements from each of the walkability model subcategories are featured in the new framework, undoubtedly an alternative opinion might have ordered the framework differently. Figures 8 and 9 on page 35 give an indication of where each walkability subcategory from the theory was considered in the new framework.
In summary this section devised an accessibility framework from the walkability literature. In the next section the framework will be used to assess how the theory of pedestrian accessibility is considered in current pedestrian design practice in Central Wellington. 
A c cessibilitity

\begin{tabular}{|c|c|c|c|c|}
\hline $\begin{array}{l}\text { Proximity and } \\
\text { Legibility }\end{array}$ & $\begin{array}{l}\text { Vehicle safety and } \\
\text { (CPTED) }\end{array}$ & $\begin{array}{l}\text { Path quality and } \\
\text { comfort }\end{array}$ & $\begin{array}{l}\text { Connectivity } \\
\text { (Macro Urban } \\
\text { scale) }\end{array}$ & Interest \\
\hline $\begin{array}{l}\text { - The potential for } \\
\text { the environment or } \\
\text { element to disrupt } \\
\text { path continuity. } \\
\text { - The environment or } \\
\text { element's effect on } \\
\text { journey distances } \\
\text { with regards to } \\
\text { time and physical } \\
\text { length. } \\
\text { - The environment or } \\
\text { element's effect on } \\
\text { user perceptions of } \\
\text { distance. } \\
\text { - How the } \\
\text { environment or } \\
\text { element interferes } \\
\text { with the users } \\
\text { awareness of the } \\
\text { path's existence. }\end{array}$ & $\begin{array}{l}\text { - The presence of } \\
\text { social safety offered } \\
\text { by the environment } \\
\text { or element. } \\
\text { - How the spatial } \\
\text { composition of the } \\
\text { environment affects } \\
\text { Crime Provention } \\
\text { Through Environ- } \\
\text { mental Design } \\
\text { (CPTED). E.g the } \\
\text { extent of evening } \\
\text { lighting offered in } \\
\text { the environment. } \\
\\
\text { - How the spatial } \\
\text { composition of the } \\
\text { environment or } \\
\text { element affects the } \\
\text { pedestrian to vehicle } \\
\text { interface. }\end{array}$ & $\begin{array}{l}\text { - How the quality of } \\
\text { the path surface } \\
\text { /ergonomics affects } \\
\text { user mobility. } \\
\text { - How the } \\
\text { environment or } \\
\text { element affects } \\
\text { spatial comfort in } \\
\text { terms of: } \\
\text { - Buffering from } \\
\text { vehicles. } \\
\text { - Noise/air quality. } \\
\text { - Shelter from } \\
\text { weather. }\end{array}$ & $\begin{array}{l}\text { - Characteristics of } \\
\text { the land use } \\
\text { pattern in terms of: } \\
\text { - Density } \\
\text { - Building and land } \\
\text { use. } \\
\text { - The network's } \\
\text { treatment of } \\
\text { significant } \\
\text { pedestrian barriers } \\
\text { such as land } \\
\text { features and large } \\
\text { roads. } \\
\text { - Connectedness } \\
\text { between paths } \\
\text { within the network. }\end{array}$ & $\begin{array}{l}\text { - The extent of } \\
\text { engagement with } \\
\text { the surrounding } \\
\text { context in the form } \\
\text { of views or physical } \\
\text { connectedness. } \\
\text { - The extent of social } \\
\text { interest offered in } \\
\text { the environment. } \\
\text { - The extent of built } \\
\text { interest in the form } \\
\text { of sculpture, or } \\
\text { architecture that is } \\
\text { offered in the } \\
\text { environment. } \\
\text { - The extent of } \\
\text { natural interest in } \\
\text { the form of } \\
\text { vegetation or } \\
\text { scenery that is } \\
\text { offered in the } \\
\text { environment. }\end{array}$ \\
\hline
\end{tabular}




\begin{tabular}{|c|c|c|c|c|}
\hline $\begin{array}{c}\text { Proximity and } \\
\text { legibility }\end{array}$ & $\begin{array}{c}\text { Safety and } \\
\text { CPTED }\end{array}$ & $\begin{array}{c}\text { Path quality and } \\
\text { comfort }\end{array}$ & $\begin{array}{c}\text { Connectivity } \\
\text { (macro) }\end{array}$ & Interest \\
\hline 1 & 4 & 5 & 1 & 6 \\
\hline 10 & 9 & 8 & 2 & 7 \\
\hline 11 & & & 3 & 13 \\
\hline & & & 12 & \\
\hline
\end{tabular}

\subsection{Current Pedestrian Design Practice in}

\section{Central Wellington.}

Figure 8: How Southworth, Alfonzo, and Metah's, walking models are distributed in the new framework for pedestrian accessibility in Figure 7.

\begin{tabular}{|l|c|}
\hline $\begin{array}{c}\text { Southworth's } \\
\text { model }\end{array}$ & Code \\
\hline Connectivity & 1 \\
\hline $\begin{array}{l}\text { Linkages to } \\
\text { other modes }\end{array}$ & 2 \\
\hline $\begin{array}{l}\text { Fine grain and } \\
\text { varied land use } \\
\text { pattern }\end{array}$ & 3 \\
\hline Safety & 4 \\
\hline Quality of path & 5 \\
\hline Path context & 6 \\
\hline
\end{tabular}

\begin{tabular}{|l|c|}
\hline \multicolumn{1}{|c|}{$\begin{array}{c}\text { Alfonzo's } \\
\text { model }\end{array}$} & Code \\
\hline Pleasurability & 7 \\
\hline Comfort & 8 \\
\hline Safety & 9 \\
\hline Accessibility & 10 \\
\hline Feasibility & 11 \\
\hline
\end{tabular}

\begin{tabular}{|l|c|}
\hline \multicolumn{1}{|c|}{$\begin{array}{c}\text { Metah's } \\
\text { model }\end{array}$} & Code \\
\hline Usefulness & 12 \\
\hline $\begin{array}{l}\text { Sense of } \\
\text { belonging }\end{array}$ & 13 \\
\hline
\end{tabular}

Figure 9: Summarised

Table of Southworth,

Alfonzo, and Metah's,

walking Models.

The pedestrian design practice in Central Wellington will be looked at in two parts:

- That the main documents used to design pedestrian infrastructure in Central Wellington demonstrate an incomplete understanding of how the built environment affects accessibility.

- The problems with the current pedestrian design process in terms of failing to assess in pedestrian infrastructural planning a key area of Central Wellington; and also, the problems with the current techniques used to identify what, and where, are the barriers to walking in the city.

\subsection{1 (Part 1) Pedestrian Design in Wellington:}

A Lack of Understanding of the Relationship Between Pedestrian Accessibility and the Built Environment.

The previous chapter highlighted that it is important to understand accessibility in the built environment in order to understand how people make travel decisions. Subsequently, this discussion will examine how 
pedestrian accessibility is currently considered in pedestrian design practice in Central Wellington. The following list is a record of national and Wellington regional policy documents that form the basis for pedestrian design practice in Central Wellington. Note the most detailed document in this list is the New Zealand Transport Agency's (NZTA) Pedestrian Planning and Design Guide (PPDG), as it contains the 'best practice' design guidelines for designing in the pedestrian realm.

- Wellington walking policy

(WCC Wellington Walking Policy)

- Walking and Cycling Regional Walking Plan

(GWRC Walking and Cycling Regional Walking Plan)

- The Pedestrian Planning and Design guide (NZTA)

\section{- Wellington District plan Guidelines}

(WCC Wellington City Council District Plan)

This discussion will review the three most relevant documents relating to pedestrian design practice in Central Wellington (indicated in bold in the above list). A table was used to conduct the review (Appendix E pg 226) however, refer to Figure 10 as an abstract representation of this table. The table allowed each of the spatial components/typologies/principle guidelines, from the pedestrian design documents, to be assessed in terms

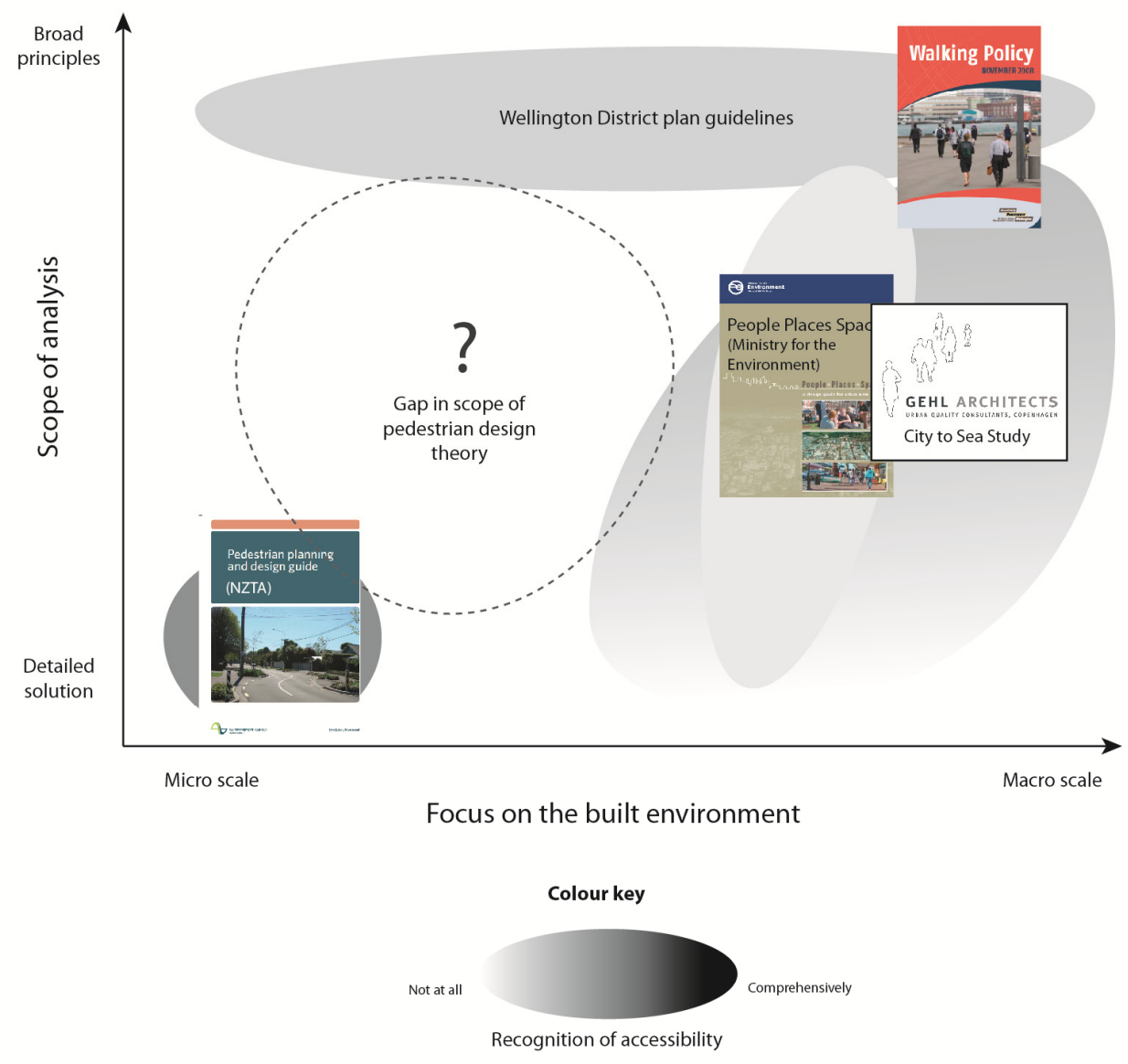

Figure 10: Representational diagram showing the scope of various pedestrian design theory and guidelines in Central Wellington. 
of their consideration for pedestrian accessibility. From this analysis two issues were highlighted about the documents.

1. Firstly, the documents lack a spatial understanding of how pedestrian accessibility barriers are created through the design of the built environment. This is significant because these are the negative spatial characteristics that need to be avoided when designing in the built environment, as they affect pedestrian travel behaviour negatively.

2. Secondly as a consequence, this raises a concern about the subsequent effect this problem has on the promotion of walking patronage in New Zealand.

However, before these above two problems are discussed one further comment needs to be made about the process of reviewing Central Wellington's pedestrian design practice. Pedestrian planning and design practice is broken into two parts:

- Policy, which operates in a 'theories' medium, setting the overarching framework for how pedestrian design should be implemented in practice.

- Design guides, which operate in a theoretical spatial medium mediating between the policy and real world practice.

This thesis argues that a successful pedestrian design process needs to perform well in both the policy and the design guides. Therefore distinguishing between these parts is important when reviewing how accessibility is considered in pedestrian design practice in Central Wellington.

The Lack of Understanding Regarding Pedestrian Accessibility

Firstly, the lack of understanding in the PPDG of how the design of the built environment affects pedestrian accessibility, limits how effective improvements in the pedestrian network can be in increasing pedestrian accessibility. At a micro scale (down at street level from the perspective of the pedestrian) the PPDG categorises the pedestrian environment into the following:

- Usability of the footpath - including, quality of the footpath surface, and the amenity benefits of landscaping and street furniture that occur along the path;

- $\quad$ Lighting sufficiency;

- Signage;

- Intersections with the vehicle network.

Each of the categories is then broken further into subcategories containing built elements such as driveways, landscaping etc, as shown in the table (Appendix E pg 226). These built elements are important in terms of pedestrian accessibility, however this thesis argues the variety of categories mentioned in the PPDG is limited. Furthermore the PPDG's 
method of simplifying the pedestrian realm has caused other important environmental elements in the surrounding context to be excluded. Subsequently, the insufficient range of pedestrian design elements featured the PDDG is significant as it limits how far accessibility in the pedestrian environment can be understood. For example, if we look at the spatial elements that affect pedestrian accessibility in the PPDG: The footpath, shared paths, grade separation, ramps and steps; all these elements only consider the spatial conditions of the immediate footpath. As a result, the PPDG does not recognise the broader environment conditions that occur beyond the footpath boundary such as the different types of buildings and land uses. This thesis argues this is significant because in a pedestrian's journey there is a wide range of land use and building 'typologies' outside the footpath boundary and these contribute to a pedestrian's perception of accessibility. For example you may walk certain routes over others because they have more interesting housing facades, perhaps are more sensuous, or comfortable to walk past due to vegetation; or perhaps because there is a possible shortcut by cutting through informal areas like car parks, schools, etc. All these reasons are examples of the environmental conditions that occur outside of the immediate footpath that affect the way pedestrians travel, however they are not considered in the PPDG.

\section{How Each Spatial Element is Assessed against Accessibility}

The second criticism of the PPDG is that each of the spatial elements such as footpaths or shared paths are not assessed thoroughly against all the sub categories that affect accessibility in their assessment of each design elements. This is demonstrated clearly by checking the table again (appendix E pg 226), which assesses each spatial element from the PPDG, against the criteria for accessibility derived from the literature. The table indicates there is a strong focus on the accessibility criteria of 'path quality'; this includes the usability of material surfaces, the ergonomic dimensions; and also the criteria of personal 'safety' in terms of the 'pedestrian vs. vehicle interface' and also 'Crime Prevention Through Environmental Design' (CPTED), which is covered by an independent document by the (Ministy of Justice) National Guidelines for Crime Prevention through Environmental Design in New Zealand. However the table indicates there is little recognition of the all other accessibility sub categories of: comfort, interest, connectivity, and 'proximity and legibility', which need to be considered when designing for pedestrian accessibility in the built environment. This is problematic considering the literature on pedestrian travel behaviour highlighted that pedestrian behaviour in the built environment is a reaction to their perception of accessibility in regards to walking. The consequences of this can be shown through an example using the built element of the 'footpath'. The footpath is a primary way pedestrians move through the built environment. Therefore at a micro scale (at street level to the pedestrian) the material quality, 
lighting, and usability of this path is important, and as a pedestrian you may walk certain streets over others because of these factors. However, now consider the accessibility subcategory of 'comfort' in terms of shelter from weather, which is a subcategory not considered in the PPDG. When it is raining you will probably take a route because it is more sheltered than others, perhaps it is even a longer route. However, in the evening what if the same sheltered route is secluded or quiet and feels dangerous due to an absence of people on the street? In order to be in a safer area where more people are present, you might have to walk an alternative unsheltered route home. This example demonstrates how an accessibility subcategory that is not considered in the PPDG, 'comfort through shelter from weather', can affect a pedestrian's travel behaviour. This example therefore highlights that:

- It is vital to analyse each design element against all subcategories of pedestrian accessibility;

- The PPDG cannot give a complete understanding of how pedestrian travel behaviour is affected by the design of the built environment, or how this can be addressed spatially through design.

As a final point, this thesis argues that the two problems with the pedestrian design documents have occurred due to accessibility in the policy theory failing to be translated sufficiently into the spatial design guides. The policy for pedestrian design in Central Wellington demonstrates some understanding of the importance of accessibility in the built environment. For example there are a number of policy objectives within the Wellington Walking Policy (WCC) that address some of the subcategories of the pedestrian accessibility framework such as:

- $\quad$ Objective 2 - improve pedestrian safety through the city (WCC 9),

- $\quad$ Objective 7 - increase the number of walking trips (WCC Wellington City Council 21).

- And also in the Greater Wellington Council's Regional Walking Plan (GWRC Greater Wellington Regional Council;). The plan outlines a series of actions to be taken when conducting a review of the pedestrian network. Such as improving: footpath surfaces, obstructions, personal safety, aesthetics, shelter, route directness and connectivity, signage, road safety etc. (WCC Wellington City Council 4).

All these components are relevant to pedestrian accessibility, however, although accessibility is well recognised in policy as a 'theories' medium, unfortunately it fails to be translated into the spatial medium as design guidelines. This was highlighted in the Pedestrian Planning and Design Guide as the subcategories of accessibility, which were established in the framework for accessibility in section 2.3.2, were not all recognised in the guides. 


\subsection{2 (Part 2) Criticisms of Pedestrian Design Process in}

\section{Wellington}

There are two different approaches used by Local Authorities to research, design, and implement, pedestrian infrastructure in Wellington Central. These are:

- The 'top down' expert approach, such as 'The City to Waterfront study' by Copenhagen urban design consultants (Gehl Associates).

- And the 'bottom up' approach 'Community Street Audits' by the NZTA.

Both approaches are subject to criticism and will be discussed in detail beginning with Gehl's top down approach.

\section{'Top down' approach: Gehl Associates}

The purpose of the 'The City to Waterfront study' was to understand how Wellingtonians use public space in the city, how they commute, and also to provide some suggestions as to how the city's public spaces could be improved. This thesis considers 'The City to Waterfront study' as a 'top down' expert approach to improving pedestrian accessibility, the approach is problematic as it fails to identify three key issues:
Firstly, what the public consider to be the most significant barriers to walking in the built environment, as well as where they are located and the spatial conditions that have led the barriers to occur in the first place. As a result Gehl's designs lack focus in addressing the key barriers to walking, resulting in the design interventions that are less effective. The cause of this problem is that Gehl's approach to understanding the site is quantitative and not qualitative. His approach uses expert analysis to make educated judgments as to how the city can be improved. For example Gehl's approach has a heavy reliance on using pedestrian counts to locate what areas in the city are less used by pedestrians, and therefore need improving. This thesis argues this technique is problematic, as pedestrian counts do not explain what areas of the built environment pedestrians consider to be barriers to walking.

Secondly, Gehl's approach provides an overall framework of direction to improving the quality of the pedestrian environment. For example his approach outlines spatial principles such as:

“Step up pedestrian priority city wide... or... Introduce more walking routes with pedestrian priority etc (Gehl Associates 50).”

However, this approach is limited because the spatial principles are broad, and therefore fails to provide a detailed solution to Wellington's walking 
problems. This is problematic because in practice the designer then can only refer to the guidelines within the PPDG, which were proven earlier to have an incomplete understanding of pedestrian accessibility.

Thirdly Gehl's study is limited in scope in that it only addresses the CBD area. This is problematic as it causes the area between the city's residential catchments and the CBD to be neglected (Figure $3 \mathrm{pg} 10$ ). This same limited scope is also evident in a more recently commissioned report by the Central Wellington local authorities, The Space Syntax City Centre Movement Infrastructure Analysis, which focused specifically on the CBD. This thesis argues the area joining the residential catchments to the CBD must be addressed in order to improve pedestrian accessibility throughout the wider pedestrian network. In this sense, both Gehl's and Space Syntax's reports expose a gap in Wellington's pedestrian infrastructure planning.

\section{'Bottom up' approach: NZTA Community Street Audit}

Contrasting to the 'top down' expert approach of Gehl is the NZTA's 'bottom up' Community Street Audit. The Community Street Audit is used by local authorities to find out which streets within the city are underperforming from the perspective of the pedestrian. The audit's method involves members of the community carrying out a walkthrough survey within a site in order to review the sites 'walkability'. The principle of this method is successful because it reaches a conclusion based on consultation with the public on their perceptions of walking in the built environment. However, the technique used to carry out the assessment is limited:

1

Firstly, the on-site walkthrough method requires significant organisation of the survey participants. Thus the method is time consuming, resource heavy, and limited to small-scale analysis, or as far as people are prepared to walk. This is a significant limitation because the method is not suitable for large census scale analysis, or as a way of identifying pedestrian barriers across the city on a macro scale; both of which are necessary in order to fully understand the pedestrian barriers present in a city's built environment.

2

Secondly, the survey technique used to question the respondents uses perception but is not cognitive. This is evident in two ways: Firstly, the survey questions are prescriptive in that they force participants to answer a range of questions as prescribed by the interviewer. As a result, participants are not given the opportunity to identify the barriers they consider most significant, therefore causing the technique to be less accurate resulting in the design outcomes being less efficient. Secondly, behavioural theory says both perception AND cognition affect behaviour, therefore, in order to understand a behavioural problem, both need to be 
considered. Thus the technique of using on site walk-throughs is problematic as it relies on perception and observation to identify barriers in the environment, and as a result doesn't allow a respondent to recall barriers through the mental process of cognition. This is also dangerous because relying only on user perception of the environment, without considering a person's cognitive understanding of the environment, can distort the accuracy of the barrier analysis. For example respondents are able to search the surroundings at the time of the interview to identify any objects/spaces as barriers, thus placing a strong emphasis on using perception to identify barriers as appose to cognition.

Thirdly, the approach is limited to only improving accessibility at the micro scale. There are two reasons for this: The approach operates within the context of the footpath, for example improving the quality of the footpath surface, and road crossings etc; and therefore has no broad consideration of how accessibility can be improved at the larger macro scale. Also, as mentioned previously, is limited because the walkthrough approach is not suitable for large-scale analysis across the city.

In conclusion of this analysis, two important improvement need to be made to the pedestrian design process in Wellington:

\section{Improvement One}

Firstly, to gain a better understanding of how pedestrian accessibility is affected by the built environment, higher order frameworks such as the PPDG need to:

1. Acknowledge the complete spectrum of pedestrian accessibility criteria outlined in the literature, therefore ensuring a thorough level of analysis when assessing each pedestrian typology, in terms of its affect on accessibility.

2. Be familiar with a wider range of built elements that affect pedestrian accessibility within the built environment, such as those that occur outside of the immediate footpath, as well as their spatial conditions that affect accessibility. Note in order to achieve this further research needs to be undertaken to reveal a wider range of design elements that are present in the surroundings, which were not identified in the PPDG.

3. In addition to researching the pedestrian access typologies at the micro street scale, there needs to be an awareness of how accessibility is also affected by the environment at the macro scale across the city.

\section{Improvement Two}

In terms of the overall method used in the current pedestrian design process: 
1. A wider analysis of the pedestrian network needs to be considered in Wellington's pedestrian infrastructure planning, which includes the area between the outer residential catchments and the CBD.

2. A new more effective method of identifying barriers in the built environment needs to be designed using cognitive and perception theory, as this theory is crucial in understanding people's travel behavioural decision-making

The following section will look at a key strategy for achieving a balanced 'top down' and 'bottom up' approach when researching why people make travel behavioural decisions in the environment. This thesis argues that this strategy, if adopted, would improve the current methodology for designing pedestrian infrastructure in Central Wellington.

\subsection{Forming a New Pedestrian Design}

\section{Methodology}

This section looks at research by Taylor, Zube and Sell, who have developed a robust strategy for carrying out perception research in the built environment. The theory is significant because it will be used, along with the other findings from this chapter, to form the initial 'Hypothesis Pedestrian Design Methodology' for implementing pedestrian infrastructure in Wellington. The hypothesis methodology is tested through a series of experiments later in this thesis. Because Taylor, Zube and Sell's strategy is centred around the theory on perception research, it is important this discussion begins by recapping on what role perception research has when resolving travel behavioural problems.

\subsubsection{Perception Research}

'Perception' of accessibility was highlighted earlier in this chapter as the fundamental principle affecting pedestrian travel behaviour. For example, Feimer states environmental perception is the central research issue in Environmental Psychology (61). Perception is an important part of the human and environment transaction, 
"providing the link between the physical context and subsequent cognitive, affective, and behavioural response (61)."

Therefore understanding how pedestrians perceive accessibility in the built environment is a crucial step towards designing more accessible pedestrian environments. The following research by Taylor, Zube, and Sell looks at some of the problems faced when carrying out perception research.

\subsubsection{An Environmental Perception Framework}

The research identifies and critiques the four paradigms that exist within perception research techniques exploring the relationship between humans, landscape, and their resultant interaction process. Their research is important because it develops a new research strategy that reduces the unwanted variability that is often present in perception research, by achieving the right balance of 'top down' versus 'bottom up' approaches. Their research is significant to this thesis as it can be used to create a new strategy for researching and understanding how pedestrians' experience accessibility in the built environment. The four paradigms are represented in the table Figure 11 and are discussed below (Taylor, Zube and Sell 361389):
Taylor, Zube, and Sell argued that each research paradigm could be critiqued for its research intent. On one hand the Expert and Psychophysical approaches seem to be focused on achieving utility in their results as a trade off for easier application. This is evident in the techniques used in the approaches such as: in the expert approach a trained expert makes educated judgments about what in the environment they think is significant to the public users; in the Psychophysical approach public perception of new environments is predicted in order to bypass further public research. The consequence of this is twofold:

- Firstly, it has been found that expert assessment as a way of representing greater population views is inaccurate due to a "sensitivity differential” between opinions of professionals and non-professionals (Feimer 75).

- Secondly, bypassing public opinion causes the research information to become simplified as subtle and local knowledge is lost (Taylor, Zube and Sell 387).

On the other hand, Cognitive and Experiential approaches seek out the grassroots knowledge of the public's understanding, in particular how public values of the environment are formed through human and environment interaction (Taylor, Zube and Sell 388). This is beneficial because the Cognitive and Experiential approaches essentially gather more diverse variations of perception knowledge; however, this is also their shortfall as the subtlety of the information is often subjective to 


\begin{tabular}{|c|c|c|}
\hline & Description & Weaknesses \\
\hline $\begin{array}{c}\text { Expert } \\
\text { assessment }\end{array}$ & $\begin{array}{l}\text { - Description of the landscape from the view of a } \\
\text { trained observer. } \\
\text { - The assessment embodies attributes that are } \\
\text { adopted from the expert's profession, and, can be } \\
\text { altered by the profession through design. }\end{array}$ & $\begin{array}{l}\text { - } \begin{array}{l}\text { Professionals notice superfluous attributes } \\
\text { that are 'un-noticeable' to the general public. }\end{array} \\
\text { - } \quad \text { Subjective to the expert. This causes an issue } \\
\text { when designing for the public's needs. }\end{array}$ \\
\hline Psychophysical & $\begin{array}{l}\text { - Predicting public perception of new environments } \\
\text { by drawing on past ratings made by the public. } \\
\text { These ratings are "obtained from controlled, } \\
\text { experimental manipulations of landscape views, or } \\
\text { landscape surrogates or simulations (387)." }\end{array}$ & $\begin{array}{l}\text { Rating scales are often developed by } \\
\text { professionals and therefore reflect their } \\
\text { intentions, not the perception of the public. }\end{array}$ \\
\hline $\begin{array}{c}\text { Cognitive } \\
\text { assessment }\end{array}$ & $\begin{array}{l}\text { - Understanding why the public subconsciously value } \\
\text { selected aspects of the landscape, and why are they } \\
\text { important to them. } \\
\text { - How meaning becomes assigned to the landscape } \\
\text { through experience. }\end{array}$ & - $\quad$ Subjective to the respondent. \\
\hline Experimental & $\begin{array}{l}\text { - How the participants' involvement in the landscape } \\
\text { forms new values. } \\
\text { - Focuses on the interaction between human and } \\
\text { landscape, and the subsequent outcome } \\
\text { - How people derive their values from the landscape. }\end{array}$ & - Subjective to the respondent. \\
\hline
\end{tabular}

Figure 11: Benefits and

Weaknesses of Taylor, Zube, and Sell's, four paradigms for perception research. 
each individual and therefore not representative of a wider population (Taylor, Zube and Sell 388). Another theory by Southworth regarding 'designing for walkability' in transportation planning, has also demonstrated the importance of a grass roots approach to the pedestrian design process. For example, three of the following five principles that Southworth argued would lead to walkability being implemented into the design of cities, were 'bottom up' approaches (254-255):

- $\quad$ Context specific understandings of walkability need to assessed in cities and suburbs - 'bottom up'

- $\quad$ Regulations need to be re-established to promote walkability.

- Walking behaviour in various social groups needs to be understood, and what aspects in the design of the pedestrian environment are most influential in assisting walking - 'bottom up'

- New innovative ways need to be designed to enhance walkability in the built environment.

- Include public involvement in the planning process - 'bottom up'

In conclusion, due to the contradicting strengths and weaknesses of each paradigm, Taylor, Zube and Sell recommend using a multi-paradigm approach when carrying out environmental perception research. The multi-paradigm strategy will be applied in the following chapter when the findings from this literature review are used to develop a 'Hypothesis Pedestrian Design Methodology', which is the foundation of the design component of this thesis.

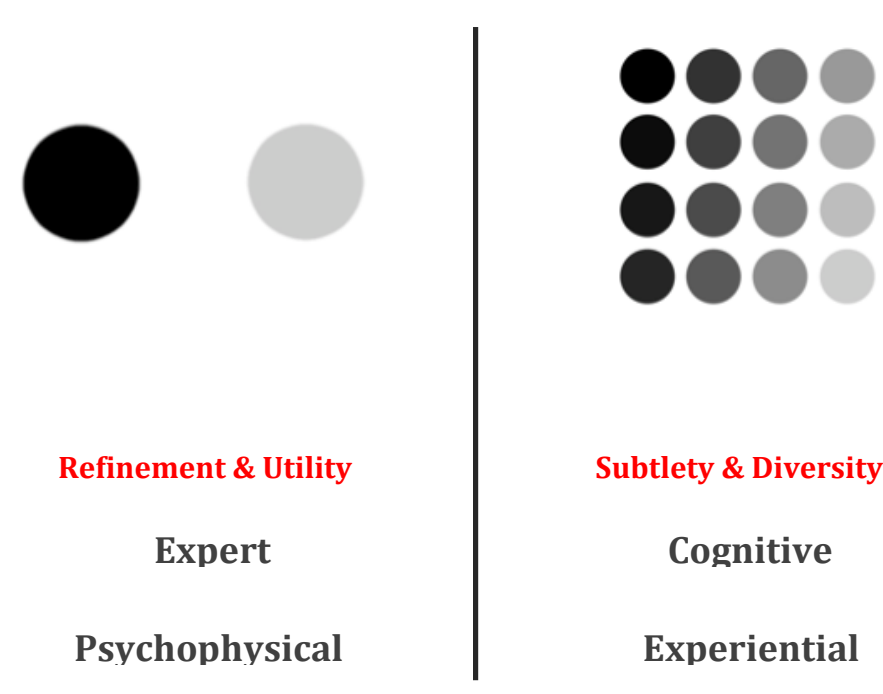

Top down

Bottom up

Figure 12: The four Research Paradigms ordered into 'bottom up' and 'top down' approaches 
paradigm approach that combines together both 'top down' and 'bottom up' approaches is most effective.

The reader should now be familiar with the summarised diagram of the The next chapter introduces the initial 'Hypothesis Pedestrian Design Methodology'.

theory introduced at the start of this chapter (Figure $5 \mathrm{pg} 22$ ). This literature review has provided insight into a number of theoretical areas, highlighting:

- Firstly, the way people understand pedestrian accessibility in the built environment, through the mental processes of perception and cognition, is the key reason affecting pedestrian travel behaviour in the built environment.

- Secondly, in the main documents used to design pedestrian infrastructure in Central Wellington there is no comprehensive spatial research explaining how the design of the built environment affects pedestrian accessibility.

- Thirdly, the current 'bottom up' and 'top down' approaches used to research, design, and implement pedestrian infrastructure in Central Wellington are subject to criticism. The literature review indicates that the approaches are inefficient when they are used separately, and therefore are problematic considering Wellington's limited pedestrian funding context.

- Fourthly, when resolving behavioural problems, such as getting people to walk more in Central Wellington, using a multi 
The literature review exposed two problems with the current way pedestrian infrastructure is researched, designed and implemented in Central Wellington, these were:

1. There is no comprehensive spatial research within the main pedestrian infrastructure documents in Central Wellington documenting how the design of the built environment affects pedestrian accessibility.

2. The current 'bottom up' and 'top down' approaches, used to design pedestrian infrastructure in Central Wellington, are not efficient considering the limited funding context in Wellington. As a result each approach is problematic when used in isolation.

\section{Chapter 3:}

\section{Methodology}

In response, an eight-staged/multi paradigm 'Hypothesis Pedestrian Design Strategy' was created as a first attempt to improve the current process for researching, designing, and implementing pedestrian infrastructure in Central Wellington (Figure 13). Over the course of the four upcoming design experiments each of the eight stages of this methodology, and their accompanying 'bottom up' or 'top down' approach, were tested, critiqued, and developed into a 'New Pedestrian Design Strategy'. The aim of this new strategy was to ensure that authorities carried out an efficient and effective process when implementing pedestrian infrastructure in Central Wellington. Note both these strategies are important to the thesis because they informed how the design research experiments were carried out. 


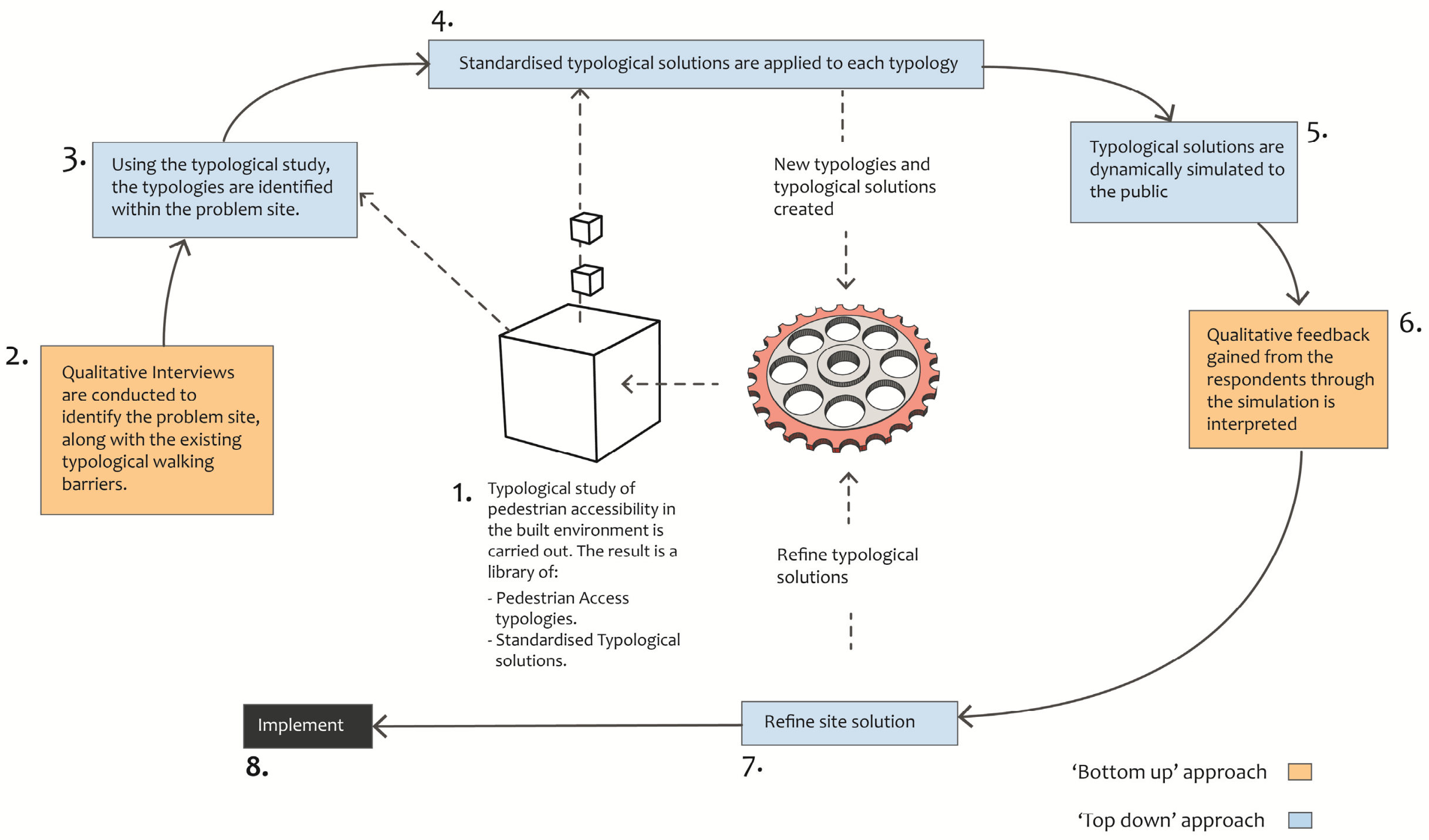

Figure 13: Initial 'Hypothesis Pedestrian Design Strategy' 


\section{The Methodology}

The following section outlines the research methodology used to carry out the design experiments in this thesis. The thesis methodology is divided into two parts, with the overall aim being to investigate how the current process for designing pedestrian infrastructure in Central Wellington can be improved, in order to increase walking patronage between Wellington's CBD and the outer residential catchments.

\section{(Part 1) Chapter 5: Understanding Site and Pedestrian}

\section{Accessibility in the Built Environment}

The methodology starts by testing the first four stages of the 'Hypothesis Pedestrian Design Strategy' (Figure 13) in order to test how successful each stage is when they are applied in practice to Wellington.

Stage 1: 'Top down' approach

- A 'top down and expert' typological analysis of pedestrian accessibility in Wellington was carried out in order to collate a library of typologies as an educative tool in improve local authorities' understanding of how the built environment affects pedestrian accessibility.
Stage 2: 'Bottom up' approach

- A pilot test was carried out using a newly developed 'bottom up' cognitive survey technique, in order to find out whether the survey method was a successful way of identifying from the perspective of the public, where the reoccurring accessibility barriers, or accessibility barrier 'hot spots', are in the pedestrian network.

Design Experiment 1

- $\quad$ The experiment trialled using the typological analysis and the cognitive surveys technique in combination, with a particular focus on whether mapping together the information obtained from the two research techniques was an effective way of researching accessibility within a site. In particular, whether this mapping technique could be used to predict where accessibility barriers are located within new sites elsewhere in the built environment.

(Part 2) Chapter 6: Designing Pedestrian Infrastructural Solutions

While the initial 'Hypothesis Pedestrian Design Strategy' was tested, each experiment presented a range of problems and new ways the strategy could be improved. In Part 2 the focus of the researched shifted away from testing this hypothesis strategy, and instead into developing a 'New 
Pedestrian Design Strategy' for implementing pedestrian infrastructure in Central Wellington. The experiment:

- $\quad$ Continues to develop the combined 'bottom up' and 'top down' technique for researching accessibility barriers within a site, by incorporating a second 'top down expert' layer of macro scale site analysis.

- Incorporates the process of improving public space in the built environment together with the process of resolving pedestrian accessibility barriers, into the one strategy for researching, designing, and implementing pedestrian infrastructure.

Design Experiment 2: Pedestrian Infrastructural solution

- This section experiments with using 'standardised typological solutions' as a way of resolving pedestrian accessibility barriers within a site. This section uncovers the limitation of using a typological approach to resolving pedestrian infrastructural problems.

\section{Design Experiment 3}

- $\quad$ Explores how having an awareness of the place-making qualities hidden within a site, enables a design intervention to act as a catalyst to improve the quality of public space in the broader pedestrian network as well as resolving pedestrian accessibility barriers on a micro scale.
Design Experiment 4

- This last experiment collates all the findings from the experiments in this chapter. The experiment tests whether the techniques and approaches that were developed throughout the previous design experiments can be combined into a one strategy for researching, designing, and implementing pedestrian infrastructure. This final strategy aims to improve pedestrian accessibility and the quality of public space within the micros scale (the 'barrier hot spot' areas), and also the macro scale (the larger urban context). 
Stages 1 and 2 of the 'Hypothesis Pedestrian Design Strategy' (Figure 13) are important for developing an understanding of pedestrian accessibility within a site. This chapter will begin by testing how these stages work individually in practice, then together in combination, in order to see whether they could be used to improve Central Wellington Local authorities' current approach for researching pedestrian accessibility within a site.

The purpose of each stage is to:

1. (Stage 1)

Carry out a 'top down' typological study of pedestrian accessibility in order to understand the spatial characteristics of the built environment that affect pedestrian accessibility.

\section{Chapter 4: Understanding Site and Pedestrian} Accessibility in the Built Environment

\section{2. (Stage 2)}

Carry out a 'bottom up' cognitive survey of pedestrian accessibility in Wellington, in order to identify what and where the accessibility barriers in the Central Wellington are. 


\subsection{Stage 1: ‘Top down’ Typological Study}

Pedestrian accessibility within a site is a complex spatial problem and is affected by many environmental elements. When trying to make sense of a complex urban environment, establishing a framework of typologies for the environment can help towards identifying, understanding, and predicting how to resolve problems that exist within it. Connolly argues,

\section{"The identification of type is the identification of the}

workings of the complexity of the real world (27)"

Note a typological analysis in this thesis refers to the term given to the process of ordering into categories the various ways pedestrians move through the built environment.

Because the real world is highly complex the utilitarian nature of a typological approach is useful because it offers a way of simplifying the complex environment into categories. The following quote by Peter Downton argues the importance of the typology/'model' in research by design.

"They are part of the core of the notion. A model is useful because it is easier to deal with than the things, events, states, processes, and relations it models. It is more comprehensible and manipulable if it is a simplification (Downton 83)."
In terms of researching accessibility, part of this thesis's aim was to gain a better qualitative understanding of accessibility in Central Wellington. However, one of the problems faced was the lack of resources, which made it un-feasible to research every persons walking experience in Wellington. I argue however that generalising the public's experiences into typological categories presents an alternative way of understanding environmentally complex problems when resources are limited. The decision to engage a typological method was therefore for three reasons.

- Firstly, typologies are a useful way of isolating the key spatial elements within the environment that directly affect pedestrian accessibility.

- Secondly, as demonstrated in the review of current pedestrian design practice in Wellington, no previous research of this detail existed about pedestrian accessibility in the built environment for Wellington.

- Thirdly, because the typological approach is a 'top down' expert approach, as a method of analysis it enables a complex environment to be researched relatively quickly when resources are limited.

\subsubsection{The Experiment Method}

The typological analysis was carried out by me, the researcher, who was performing the role of the 'expert'. The expert approach was adopted for 
its efficiency benefits as it enabled a large area of the city's built environment to be analysed under the time and resource constraints of this thesis.

The typological study began with me envisioning and documenting into typologies, from the opinion of an 'expert', the different ways a pedestrian could walk through the built environment. ${ }^{2}$ Each of the identified typologies was then assessed against the accessibility criteria from the literature (Figure $7 \mathrm{pg}$ 34). This is a significant step because unlike the NZTA assessment of typologies in the Pedestrian Planning and Design $\underline{\text { Guide, }}$ it ensured pedestrian accessibility was assessed consistently for each typology. Figure 14 and 15 are a sample of the typological study (see Appendix A pg 194 for the full typological study):

\subsubsection{Findings}

Two observations were made during the process of carrying out the typological analysis:

- $\quad$ The typologies needed be distinguished into categories of either access or land use types.

2 Note here the subjectiveness of this method, a criticism of the 'expert' paradigm stated earlier (Figure $11 \mathrm{pg}$ 45).
- There were a number of difficulties faced when translating the 'complex' real world environment into the typologies.

\section{Sub Categories Within the Typological Study}

The first observation was that the typologies could be categorised further into two groups, Access Typologies and Land use Typologies. This was because accessibility was found not only to be affected by the immediate spatial characteristics of the path that pedestrians walked on, such as ergonomics, and the presence of street planting; but also is affected by the kinds of buildings, land uses, and activities that are present in the surroundings.

\section{Access Typologies}

Spaces that allow pedestrians to move through the built environment. They physically improve pedestrian connectivity by providing routes through the built fabric and the site's natural typography.

\section{Land use Typologies}

Land uses that occur in the surrounding areas off the footpath. They create spatial conditions that affect pedestrian's experience as they move past or through a space. 
Passageway - Residential Description

A narrow path passing between residential sections. There are no entrances fronting the passageway, and the path is gener-

ally lined with property fences either side.

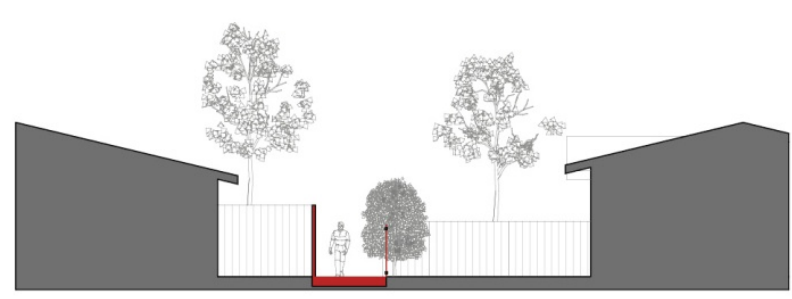

Section AA

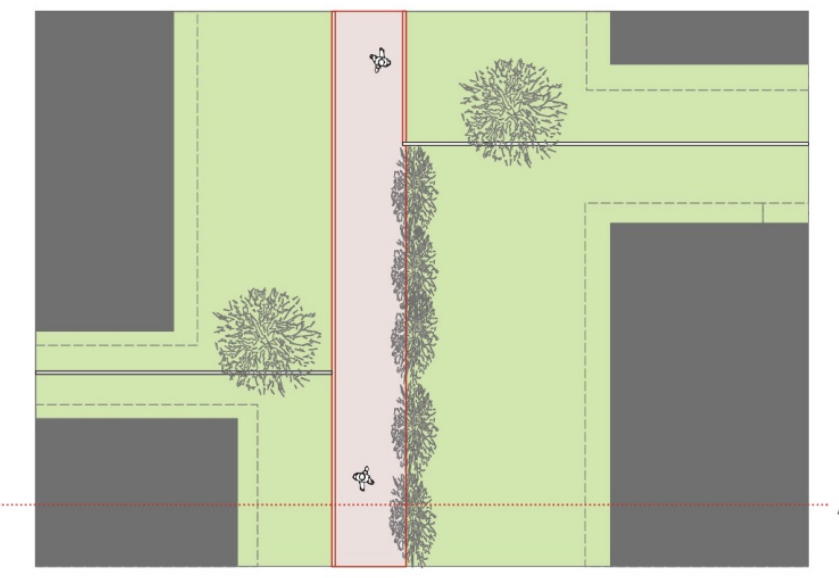

Plan
Proximity \& legibility

- Route continuity

Is reduced greatly in the

evening due to a number

of safety issues.

\section{- Route proximity}

Improves journey prox-

imity by allowng direct

streets. Essentially add-

ing fineness to the street

network thus significantly

reduces block sizes.

\section{- Legibility}

local and can

go unnoticed when not

located on a map, or are

poorly signposted.
Safety

\section{- Social safety}

Low due to absence of

people. But varies with

fence heights and eyes

on the street from sur-

rounding houses.

\section{- Evening Lighting}

Generally insufficiently lit,

particularly around den

vegetation.

\section{- Spatial design}

Bends along the path

and impressions in the

building walls can create

secluded alcoves that can

be viewed by the public

as associated with crime.

Also single entry and exit

points offer few escape

routes.

Footpath quality

(broken and overgrown)

aths can be uninviting

Characteristics of the

- Engagement with sur-

Characteristics of th

rounding context

Density

Physically restricting du

to the property bounda

to some users.

\section{- Shelter from weather}

Some shelter offered

through walls either

side of the path, and

also when vegetation is

present.

Comfort

satially dependent on

the narrowness of path

and fence height causin

the space to feel overly

confined.

Noise/air quality

Improves as the user

moves away from the

ries and fence lines. How

Building land use ever, the level of social

NA and visual engagement

depends on the height

Treatment of significant property fences permit-

barriers ting views into neigh-

Reduces block sizes.

- Connectedness bebouring properties.

-

network

Tether net

passager networks of

pasagew ays the

connectivity of the stree

network.

\section{Social interest}

None due to absence of social activity.

\section{- Visual interest}

Dependent on height of

property fences prevt of

ing views into surround-

in

degree of variation in

fence aesthetic.

\section{- Natural scenery}

Dependent on heights of property fences prevent-

ing views into prop-

erty gardens, also visible

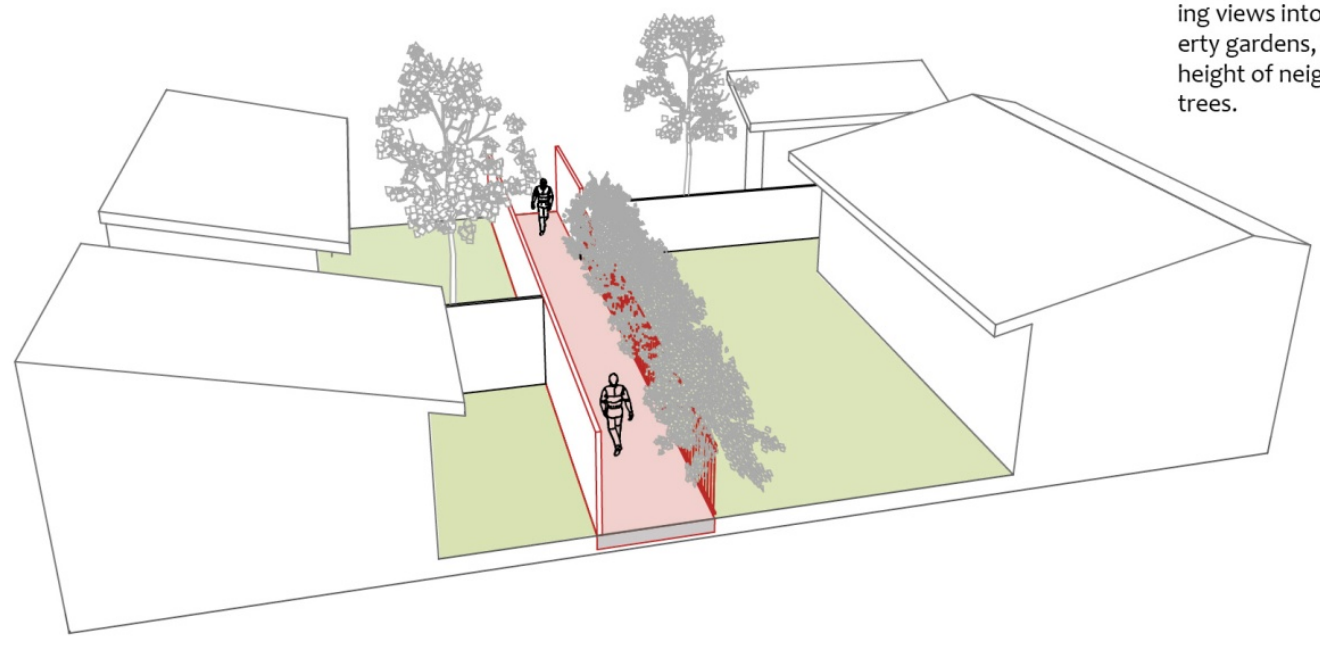




\section{Street-Residential Urban}

Description

A pedestrian and vehicle street with residential land use. Building development is of a higher density than suburban residential streets. Sections are widely covered by the building's footprint, which extend up to the footpath's edge. The footpath is narrow and has little room for vegetation and planting.

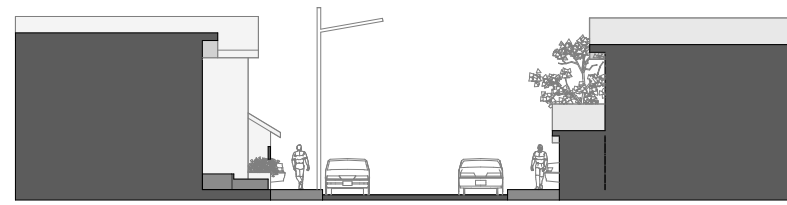

Section AA

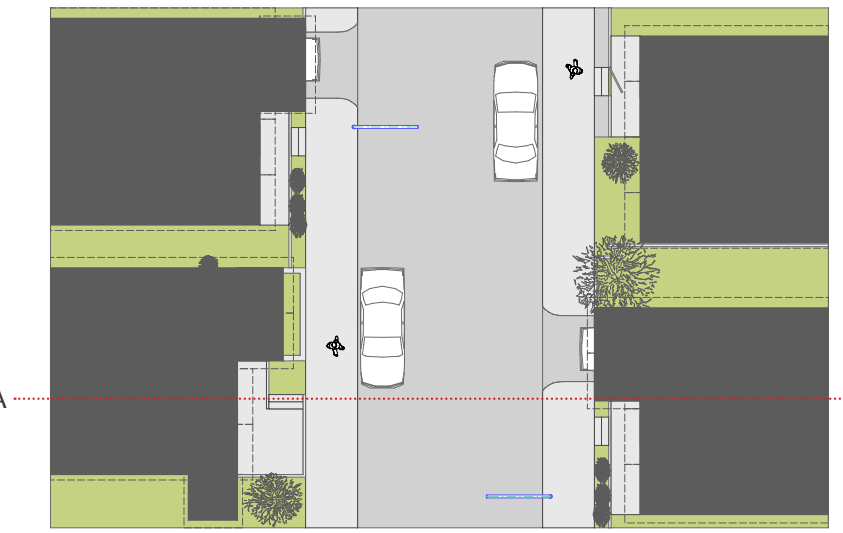

Plan
- Social safety Eyes on the street is

On some streets as a compromise for road space narrow footpaths can occur making it difficult for pedestrians to pass in both directions.

- Spatial comfort

The small scale of the street feels more intimate at street level as pedestrians are physically closer to the residential activity. However, poor vehicle-pedestrian buffering.

- Shelter from weather No shelter from the

through all hours.

- Evening Lighting

In addition to street light-

ing, nighttime lighting is

enhanced from lighting

coming from windows of

the surrounding houses.

\section{- Spatial design}

'Eyes on the street' can

be reduced significantly

if tall fences/hedges/

retaining walls, block

views into houses, or if

footpath lighting is inad-

equate.

nnectivity (Macro)

-Characteristics of the Land use pattern

Single land use means

close proximity to basic

shops or a town centre

is important for reduc-

ing trip distances. Hav

ing walkable distances

between a variety of

and uses relies on the

centres

- Treatment of significan

pedestrian barriers

- Connectedness be

tween paths in the

network

\section{- Noise/air quality}

Becomes an issue if there

is high vehicle thorough

fare as the narrowness of

the street causes pe-

destrians to be closer to

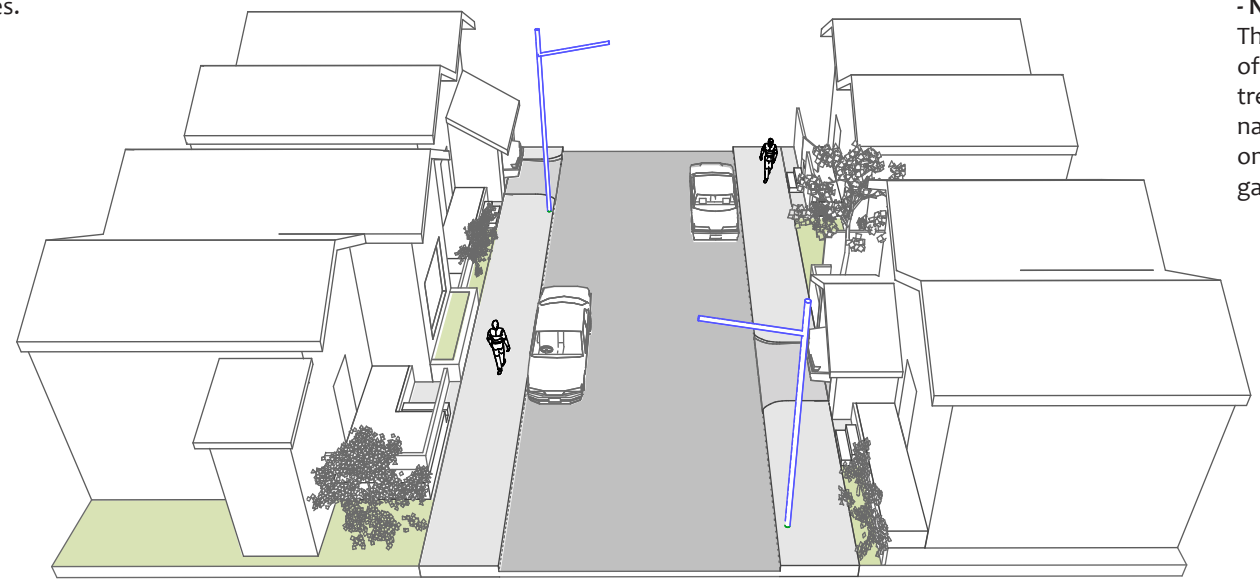

Figure 15: (Land use typology) example oute continuity The street provides pasme due to the nearby houses offering 'eyes on the street'.

Nhpute proximity

- Legibility

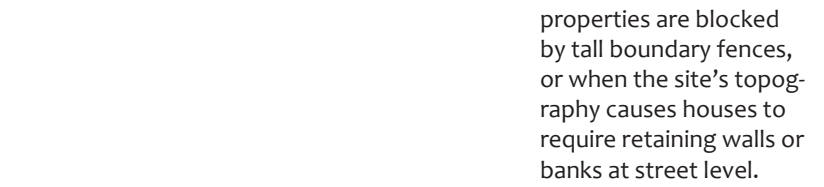

banks at street level.

- Natural Interest

The narrow street space

often results in no street

es or grass verge. Thu

(n) relies

on views into property gardens. 
Splitting the typologies into two

definitions is important because it

allows for a more detailed analysis of accessibility when analysing the built environment. For example in the access typology of a 'residential passageway' in (Figure 16), accessibility within the site is understood better if the land use typologies on either side of the footpath are analysed in addition to the access typology. In Figure 16 both scenarios, $\mathbf{A}$ and $\mathbf{B}$, fall under the access typology of 'residential passageway', however, the characteristics of the different land use typologies that occur outside of the footpath on each site, that is 'vegetation' vs. 'urban residential' land use, demonstrate different accessibility problems.

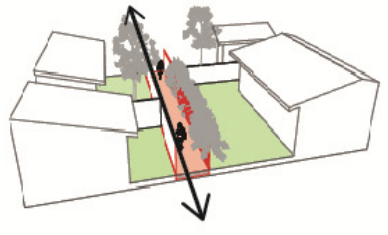

Residential passageway

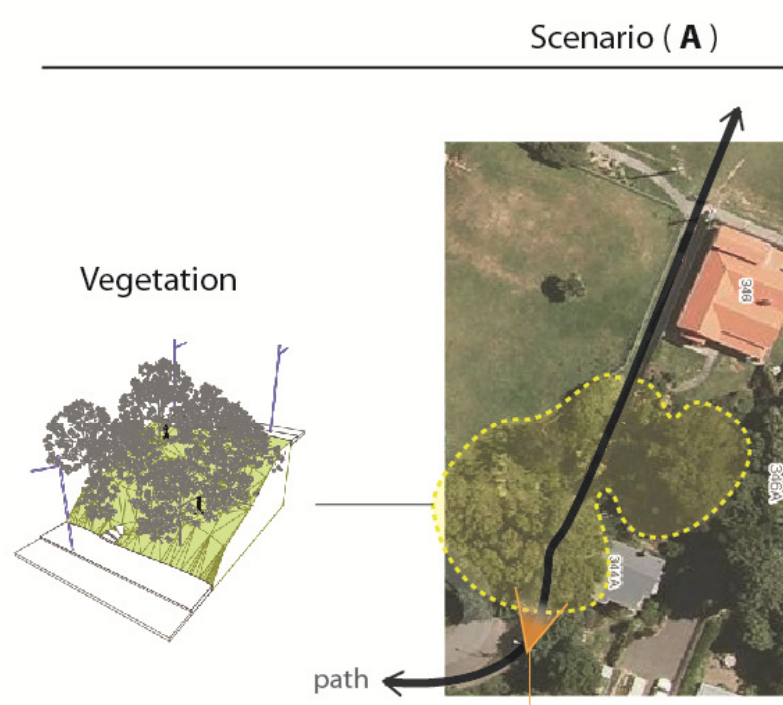

Large areas of dense deep foliage create hidden areas off the path, and blocks 'eyes on the street' from the surrounding houses.

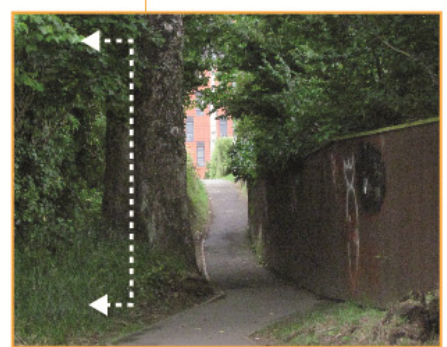

Scenario (B)

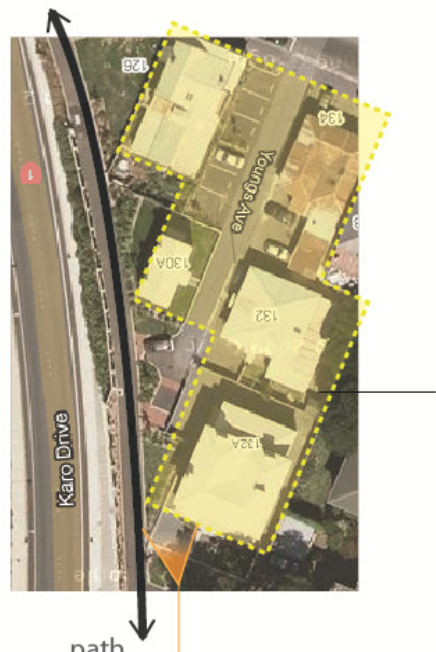

Urban residential

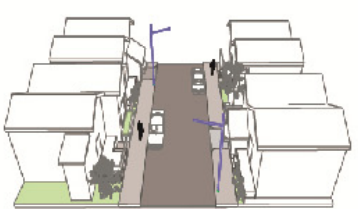

The adjacent housing properties overlook the footpath offer surveillance through 'eyes on the street' 


\section{Moving From a Text Format to a Spatial Format}

The second observation about the typological study occurred while translating the accessibility literature, which previously existed in a 'theories' medium, into the new spatial/diagrammatic medium of the accessibility typologies. What initially seemed like straightforward theoretical criteria such as, proximity, safety, connectivity etc, became complicated when tying to translate their spatial implications for pedestrian accessibility into a typology. I argue this was difficult because there is strong interconnectedness between each of the accessibility theory subcategories (proximity, comfort, CPTED, interest etc) at different micro and macro scales, which is difficult to separate. The following two examples demonstrate this:

1

If we look at the pedestrian access typology 'Residential Passageway'

(Figure 14), and consider how this typology affects the accessibility subcategory of 'proximity'. The residential passageway can improve journey proximity by adding finer connections to the street network and thus allowing more direct thoroughfare between streets. However, proximity can also be affected by other accessibility criteria. For example, if the accessibility criteria of safety in terms of CPTED is introduced. When an accessibility barrier towards safety is present along a user's most direct route to a destination, despite the 'residential passageway' in theory offering a more direct route for the pedestrian, because the pedestrian can

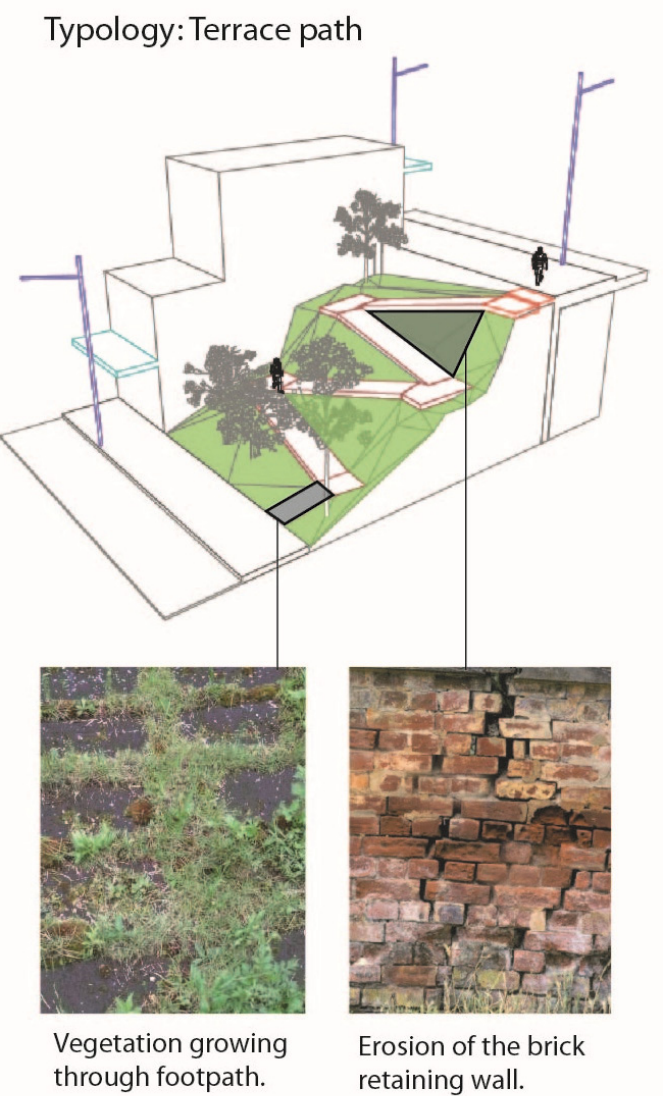

no longer use the passageway the CPTED safety barrier can remove this proximity benefit.

In a second example, if we take the access typology of an old 'Terrace Path' (Appendix A pg196) and examine it in regards to it's path quality in Figure 
17 , two contrasting interpretations can be made about the spatial qualities of the typology. On one hand the steep gradient of the path, erosion of the masonry retaining walls, and the overgrown vegetation, could be assessed as being hazardous, neglected, and in need of maintenance. However in contrast, these spatial qualities can be assessed very differently when considering their contribution towards the accessibility subcategory of 'path interest'. For example there is an appealing quality to both the eroded materiality from the worn stair treads, repaired mortar patchwork; and also the eroded, bulging and cracked masonry retaining walls. These contrasting interpretations are problematic because in the typological process the typology is unable to separate the unique interest qualities of site like age and character, from the categories of poor path quality i.e. maintenance. This is because the standardisation and utilitarian simplification of the surrounding site context is a fundamental characteristic underlying the typological process.

In conclusion of the typological study of pedestrian accessibility, despite employing a rigorous process of analysis that assessed each typology against the accessibility framework derived from the travel behaviour literature, the typological study was still flawed. This is because the study did not consult with, or factor into the analysis, any public perceptions of the built environment. This is a problem because all the relationships identified in the study between the built environment and pedestrian accessibility were strongly weighted towards the expert researcher's personal walking perceptions. In this sense I argue that relying entirely on a 'top down typological study' of pedestrian accessibility as an approach to site analysis is flawed. This is because fundamentally accessibility is a product of people's perceptions toward the built environment, and therefore is highly subjective and contextual. Thus any conclusions regarding pedestrian accessibility in Central Wellington should be derived through robust public consultation. In reflecting on this 'typological study' I acknowledge that if the research time constraints were removed, employing a 'bottom up' process of analysis in which qualitative opinions from a wider portion of the community were considered, the typological study would result in a more truthful spatial understanding of Wellingtons built environment's pedestrian barriers.

In terms of pedestrian design practice in Central Wellington, considering the lack of understanding about how the design of the built environment affects pedestrian accessibility (which was outlined in the chapter 2.4), this chapter has demonstrated that a typological analysis of pedestrian accessibility could fill this gap. This is because a typological analysis could be used to translate the accessibility theory into a spatial format that makes the travel behavioural theory more applicable to real world practice. 


\subsection{Stage 2: ‘Bottom up’ Cognitive Survey Pilot test}

Following on from the 'top down' typological study, the next step towards understanding the relationship between pedestrian accessibility and the built environment was to find out where pedestrian accessibility barriers exist in Central Wellington from the perspective of the public. This involved developing a 'cognitive survey' technique. Note the cognitive survey is 'stage 2' of the initial 'Hypothesis Pedestrian Design Strategy' (Figure 13 pg50).

To begin with, the term cognitive mapping will be explained.

\subsubsection{The Cognitive Map and Cognitive Mapping}

The term Cognitive map comes from travel behaviour theory discussed in the literature review chapter. In order to understand the concept of cognitive mapping it is helpful to recap on the following travel behaviour theory:

- When a person makes travel behavioural decisions they act according to their mental representation of the built environment; and this mental representation is formed through the mental process of cognition, which processes a person's perception experiences of the built environment over time (see Figure 5 pg 23).

The cognitive map is an important concept in understanding a person's travel behaviour as it represents a person's understanding of accessibility in the built environment. For example Mondschein, Blumenberg, and Taylor argue that although it is unclear exactly how transport infrastructure affects people's travel behaviour, research so far suggests when a person's travel actions interact with various transport infrastructure, e.g. roads and sidewalks, their experience affects their cognitive map, and subsequently their travel behaviour (849).

In order to access a person's mental representation of the environment, the idea of a cognitive map or cognitive mapping is important. This is because a cognitive map is a spatialised form of the cognitive process as a mental image (Hart and Moore 248). As a child you may have drawn 'mind maps' or cognitive maps of your journey to school, in which you drew the things along the journey that you remember. Cognitive mapping in this sense can be seen as a representational drawing of your mind's 'mental image' of the environment (Mondschein, Blumenberg and Taylor 847). 


\section{Why is Cognitive Mapping Important?}

Cognitive mapping is important for this research because it can be used in addition to the earlier typological study to understand how certain groups of people perceive pedestrian accessibility in the built environment. Therefore considering there are a number of problems with the current process Central and Local Authorities use to identify pedestrian barriers in the Central Wellington, highlighted in section 2.4.2, cognitive mapping needs to be trialled in order to see whether it could become a successful 'bottom up'/qualitative method of identifying pedestrian accessibility barriers in the built environment.

Before carrying out any survey experiments, two past examples of cognitive mapping research by Hannes and Lynch were examined in order to gain some insight into likely problems faced when using a cognitive mapping survey method. Two points were highlighted from their work:

\section{- $\underline{\text { Hannes }}$}

Hannes used the technique of combining people's recorded travel routes from the cognitive maps, with accompanying qualitative information from an additional survey. His method was useful because it allowed qualitative information about certain aspects of the respondents' cognitive maps to be teased out while they were drawing. This technique was beneficial because it meant more detailed information could be extracted from the respondents (Hannes, Janssens and Wets 76-96).

- $\quad$ Lynch

Lynch's experiment compared using verbal interviews as a survey method against the drawn cognitive mapping method. Lynch found that the cognitive mapping technique (or as Lynch calls it 'sketch mapping') had a higher threshold for depicting information than verbal interviews. This means that some elements that were highlighted in the verbal interviews did not feature on the respondents sketch map. In conclusion, Lynch expressed that it was essential to have an accompanying verbal survey working in parallel with the cognitive mapping drawing (Lynch 144).

As a final comment, Bechtel criticised cognitive mapping research as being unreliable due to much of the data within cognitive maps being uninterpretable to the researcher when viewed in isolation (95). This thesis argues however, Bechtel's criticism can be avoided. This is because the research by Hannes and Lynch suggests that by combining together cognitive sketch maps with additional verbal interviews, the researcher is able to ask questions about how to interpret the unreadable parts of the respondents' sketch maps. Similarly to Hannes and Lynch, a dual method will be adopted for the cognitive mapping surveys in this thesis. 


\subsubsection{The Experiment Method}

A pilot cognitive mapping survey was carried out first to test how successful the survey questions were at evoking the desired responses from the interviewees. Note because this exercise was only a test, just three respondents were interviewed. This pilot test was broken into two components:

1

The first component required the respondents to draw on a map of Central Wellington their journeys through the city as directed by the interviewer. The respondents were required to draw/comment on the parts of the journey they disliked and liked the most when they walked to and from home to two different locations within the survey boundary. As the respondents drew these journeys, the interviewer asked probing questions to tease out qualitative reasoning regarding each of their answers. The outcome of the survey was a map showing where each respondent perceived there to be accessibility barriers when walking, and subsequently where in the city the main problem areas are located (See Figure $19 \mathrm{pg} 71$ as an example of this map).

2

The second component was to compare the qualitative comments from the surveys, against the typologies that were present along the respondents' routes. This was to see whether there was any relationship between the typologies and the kinds of accessibility barriers that the respondents had perceived within the site. This was done using a mapping technique which represented each of the respondents' journeys in a line showing the land use typologies they walked past on either side of the footpath, as well as the access typologies they walked through. As a final step the qualitative comments made about each typology where graphed alongside this (see Figure 20 on pg71 foldout).

\subsubsection{Findings from the Cognitive Survey Pilot Test}

Three observations could be made from the pilot test:

Firstly, the pilot survey questionnaire (Appendix B 219) contained too many questions, and some questions were too open-ended, which caused the answers to be inefficient. This exposed a problem for later on when the survey was to be up-scaled and the data collated, as the questions need to be concise to avoid unnecessarily wasting resources.

2

Secondly, the level of detail respondents were able to recall and comment on about sites was often vague. For example one respondent generalised large sections of a route up to $200 \mathrm{~m}$ long as being "dark" and with "not much happening". However, only once interrogated further were they able to give more useful insight about the cause of the problems and the exact location i.e. 
"the site felt dark due to dense tree cover blocking the overhead street light" - anonymous respondent.

In contrast to this however, some respondents found it hard to expand critically on their statements. For example, to explain why certain streets or places were "scary" or felt "dirty". This difficulty appeared to be because the cognitive methodology relies on memory, and therefore causes some parts of the respondents' journeys to be better recalled than others. This variation in the accuracy of the respondents' answers is problematic as the exact origins of each accessibility barrier are needed for:

- The upcoming design experiment, which searches for a pattern between typologies on site and the identified accessibility barriers located by the respondents;

- And also in the later experiments when trying to design a solution to each accessibility barrier.

A dilemma arises here because by asking questions the interviewer has the ability to influence the respondents' answers, and thus distort the survey information. For example, Kearns argues that there is a danger in the influence the researcher has over the process of observation. Kearns argues that the researcher has an active role in the observation process whereby they can choose what and how they wish to observe (193). Thus the researcher must be aware of his or her own relationships and views towards the research field that is being studied (205).
There are two alternative solutions for improving the level of detail respondents can recall on site. The first one is to move from a cognitive survey to a site-based discussion where respondents are asked to provide walking commentary whilst they navigate the streets. This is the same technique used in the Community Street Audit used by the NZTA. However this technique is also problematic. For example Marans argues that one benefit of an on site-based discussion is that it

"Allows the interviewer to gather information about the physical setting while he or she is at the site (69)."

However, I argue that this ability to gather information from the surroundings not only applies for the interviewer, but also to the respondent. On one hand the literature highlighted that individuals' behavioural actions, such as the decision to walk or drive, is an action based on the cognitive processes, this is their mind's recollection of the perceived experiences in the environment. However in contrast, when using walkthrough site-based interviews, a respondent is able to draw attention to elements and qualities of the street environment that they might not have recalled cognitively at a desk interview. Therefore, although in a site-based interview the respondent can give more detailed answers about the areas of the surroundings that affect their experience of walking, this is less relevant. Information that respondents recall from memory through their cogitative process is key to understanding the accessibility barriers that affect a pedestrian's decision to walk. 
3

Thirdly, given the limited sample size of the pilot test, perhaps on a much larger survey the proportion of the vague comments (where respondents were unable to expand on their answers) in relation to the survey sample size would be less.

\section{Conclusions}

In conclusion to this pilot test these above points have:

- Highlighted that the interviewer has a responsibility towards the quality of research produced. It is vital that the interviewer is

familiar with the survey process and knows when, and how far, to interrogate respondents' answers when probing for more information.

- Highlighted that the information that respondents recall from memory through their cogitative process is key to understanding what accessibility barriers affect a pedestrian's decision to walk.

- Affirmed Bechtel's argument that in cognitive mapping research it is necessary to train the researchers/interviewers to ensure that there is a controlled consistency in the survey data (95).
In response to the pilot test, the following changes were made to the cognitive mapping survey method before carrying out 'Design Experiment $1^{\prime}$

- The survey questions were refined.

- The interviewer was aware to what level, and when, to interrogate the respondents to expand on their comments. 


\subsection{Design Test 1: A New Method of Site Analysis Using}

Typological Pattern Maps \& Qualitative Perception

So far it has been established that the typological study is a useful way of learning about the spatial conditions, associated with each typology, that affect pedestrian accessibility; and how these conditions could result in accessibility barriers being created in the built environment. The purpose of this next experiment, 'Design Experiment 1', was to combine the typology analysis and cognitive surveys method into one 'bottom up' and 'top down' method for locating where accessibility barriers are in the built environment, as well as the spatial principles that are causing them.

In order to do this the same mapping exercise was carried out, as in the earlier pilot test, to compare the built environment in a typological medium against the accessibility barriers raised through the 'bottom up' survey. As a research hypothesis I was interested to see whether a pattern would emerge between the kind of accessibility barriers that were being raised along a person's route, and the typologies that were present on site. Finding a pattern is significant in terms of 'stage 3 ' of the 'Hypothesis Pedestrian Design Strategy' (Figure 13 pg 50), which aims to create standardised typological solutions for each accessibility barrier, because:
- Firstly, a pattern between typologies and the barriers would expose how many typologies are in need of a standardised solution.

- Secondly, finding a pattern also suggests there is a common spatial problem associated with each accessibility barrier, and therefore makes designing a standardised solution for each barrier much easier.

\subsubsection{Method}

The method was broken into three steps:

1. Cognitive surveys were carried out to locate on a macro city scale what and where were the barriers to walking in Central Wellington as viewed by the public.

2. Next these areas where most problems occurred ('barrier hot spots') were organised into and re-represented as typologies.

3. Next the typologies were then compared against the pedestrian accessibility barriers raised by the respondents.

\section{(1) Interviews}

The cognitive surveys were carried out to achieve two goals: 
1. To locate the areas of the city where accessibility towards walking is currently viewed as a problem.

2. To understand qualitatively how the surrounding environment had caused these barriers to occur.

Due to the time consuming nature of processing each interview, only nine interviews were conducted in this experiment. Note it is important to understand that this minimal sample size does not hinder the experiment. This is because the aim was not to produce generalisable data about pedestrian accessibility in Wellington, but instead to test how the new cognitive survey methodology functioned as a way of identifying pedestrian accessibility barriers on an infrastructural scale. It is acknowledged however, that if a larger sample size were possible this methodology would be tested more rigorously. ${ }^{3}$

Site Boundary

The survey site boundary was restricted to the residential catchments surrounding the Central Wellington area (Figure 18). This area was chosen because it included the key walking distance between $1-2 \mathrm{~km}$,

\footnotetext{
${ }^{3} \mathrm{~A}$ larger survey sample would both, expose new problems when organising/interpreting the survey data, and also, result in a more democratic representation of the public's perceptions towards pedestrian accessibility in the Central Wellington.
}

which was identified in the chapter 1.05 (Figure 2 pg 9) as the threshold when a significant decrease in walking trips tended to occur. 


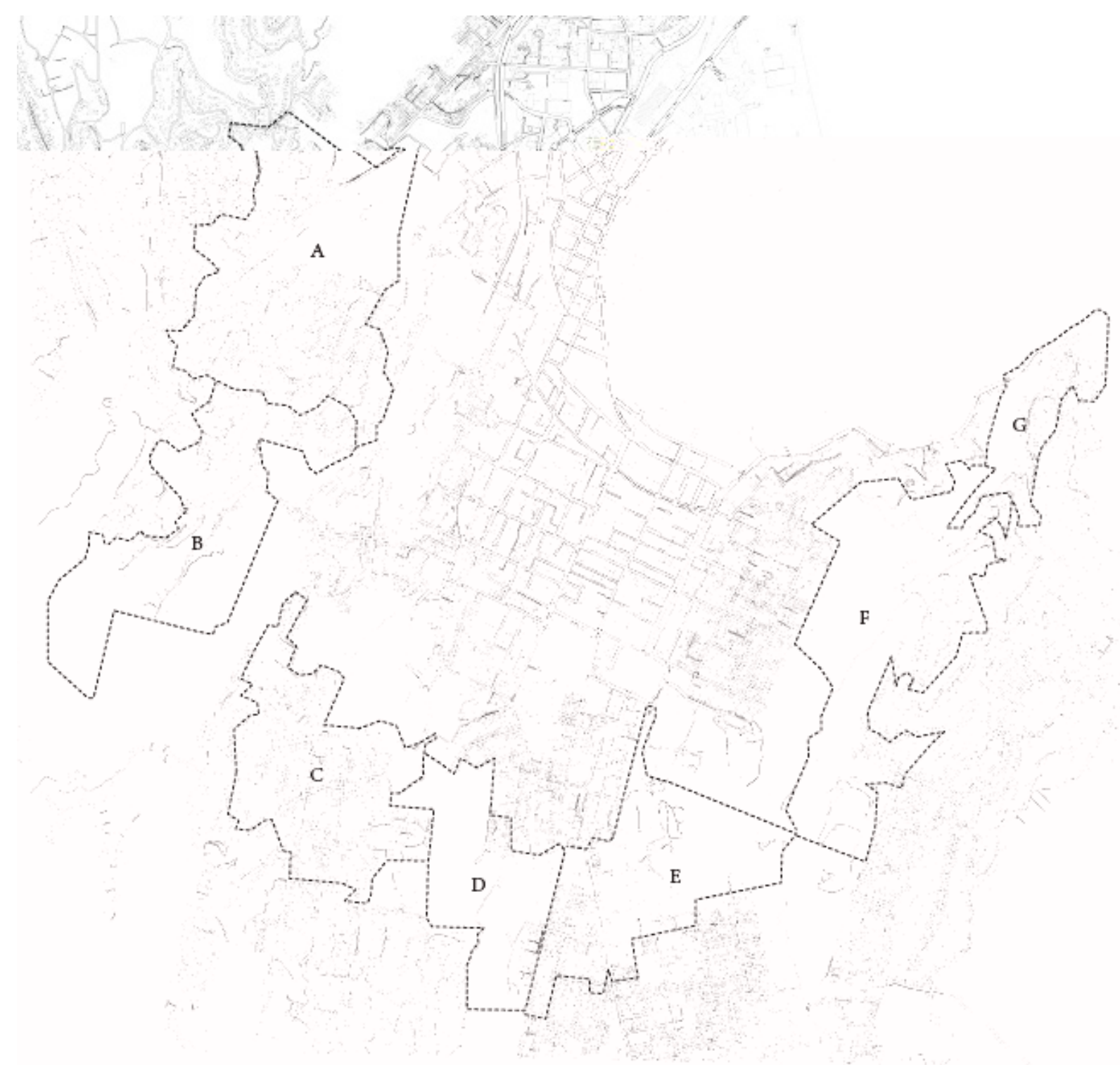

Figure 18: Map showing the outer residential catchments surrounding the CBD (not to scale) 


\section{(2) Survey Questions}

The criteria for the survey group were as follows. Respondents must:

- Have access to a vehicle and drive in Central Wellington.

- Walk within Central Wellington,

- Live within a defined area of the site research boundary,

- $\quad$ Be between the ages of 20-25.

The survey demographic was chosen because:

- They are a younger and more active age group, and therefore likely to have walked over a wider scope of the city (Ministry of Transport 6).

- For convenience reasons as it was easier for me the researcher to organise to meet with this demographic.

The interview consisted of four questions asking the respondents to draw on a large map of Wellington the journey from their homes to two different necessary activity locations - these are the activities which individuals must carry out within their lives in the urban environment, such as commuting to work, waiting for a bus, or commuting to the shops for a specific purpose (Gehl 365) -

Each survey question addressed one of the different environmental, time and weather conditions below:
- $\quad$ Night time calm weather

- $\quad$ Night time extreme weather

- Daytime calm weather

- Daytime extreme weather

Following this, the respondents were then asked to identify the places along each of these routes that they found least enjoyable (the barriers in their journey). (For more details of the questionnaire see Appendix $C$ pg220). All together 72 routes through the city were documented:

\section{9 surveys x 2 journeys to different locations x 4 varied conditions $=72$ routes}

Each survey was then overlayed onto a single map so the areas where most barriers occurred, the 'barrier hot spots', could be identified (Figure 19). The diagram shows the most intense barrier 'hot spot' was along in the area which formed a key link connecting 'residential catchment $A$ ' to the city centre. 
Figure 19: Map showing the main 'accessibility barriers hot spot' after overlapping all the respondents survey data

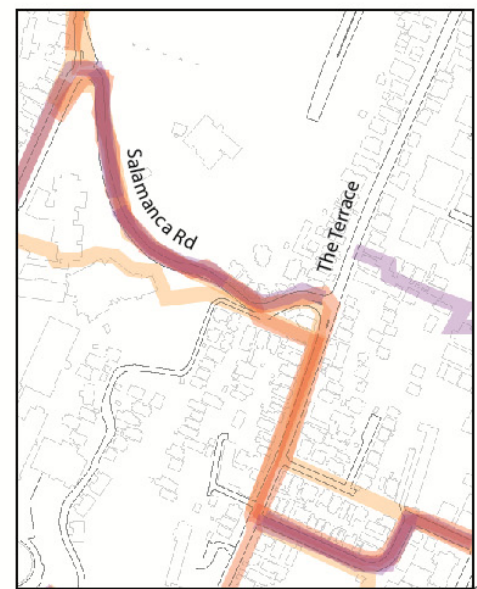

Hot spot

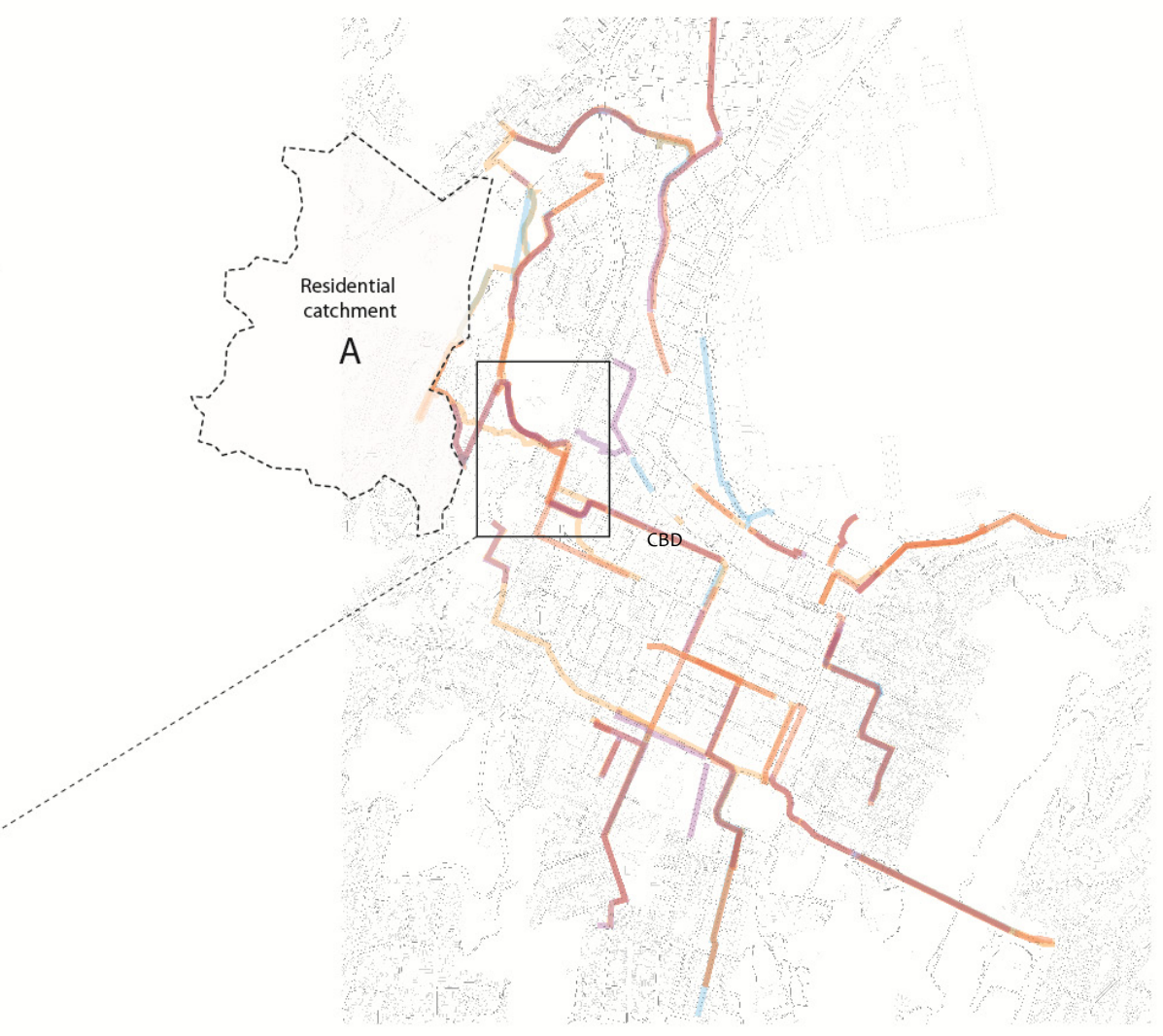

The respondents' qualitative perceptions that were recorded from the cognitive mapping interviews were then diagrammed in parallel alongside the typological representation of site. This allowed the typologies to be compared against the accessibility barriers, and any patterns to be observed (Figure 20). 
ए? 
Figure 20: Sample of mapping analysis

searching for patterns between respondents' perceptions of

accessibility barriers, and the typologies present within site 


\section{$\underline{4.3 .2 \text { Findings }}$}

Three observations were made about the use of typological pattern maps. The first two are limitations regarding the problems faced when applying the typologies as theory into practice on a real world site. These are:

- The difficulty of re-representing the complex real world environment as simplified typologies.

- The typological analysis was unable to recognise the influence of the larger urban environment on pedestrian accessibility.

The third observation is a reflection on why the 'typological pattern method' is un-successful for researching how pedestrian accessibility is affected by the built environment within a site; and as a result, why it has no further application in this thesis research.

\section{Re-representing a Site Typologically}

The first problem with the typological method of analysis was the difficulty faced when trying to align the diverse range of real world typologies within the site, with the theoretical access and land use typologies from the typological study. This was because the real world typologies were more complex than anticipated. Unlike the theoretical typologies, the real world typologies were often formed from a combination of typological spatial conditions, and as a result they were difficult to sort into obvious typology categories. This was problematic as each of these variations in real world typologies had different affects on pedestrian accessibility. For example:

\section{Example 1}

One of the positive attributes of the 'urban residential typology' (Figure 15 $p 57$ ) is the presence of 'eyes on the street', which results in a safer environment for walking after hours. However, one of the negative attributes or accessibility barriers that can arise in this typological condition is the obstructing of the 'eyes on the street'. This can occur due to a variety of reasons as demonstrated in Figure 21:

- Firstly, the sloping typography of the site can cause houses to be raised on bank walls or lowered into a gully.

- Secondly, significant garaging on the ground floor, essentially acting as a large blank wall;

- Thirdly other obstructions such as vegetation or high fences. 


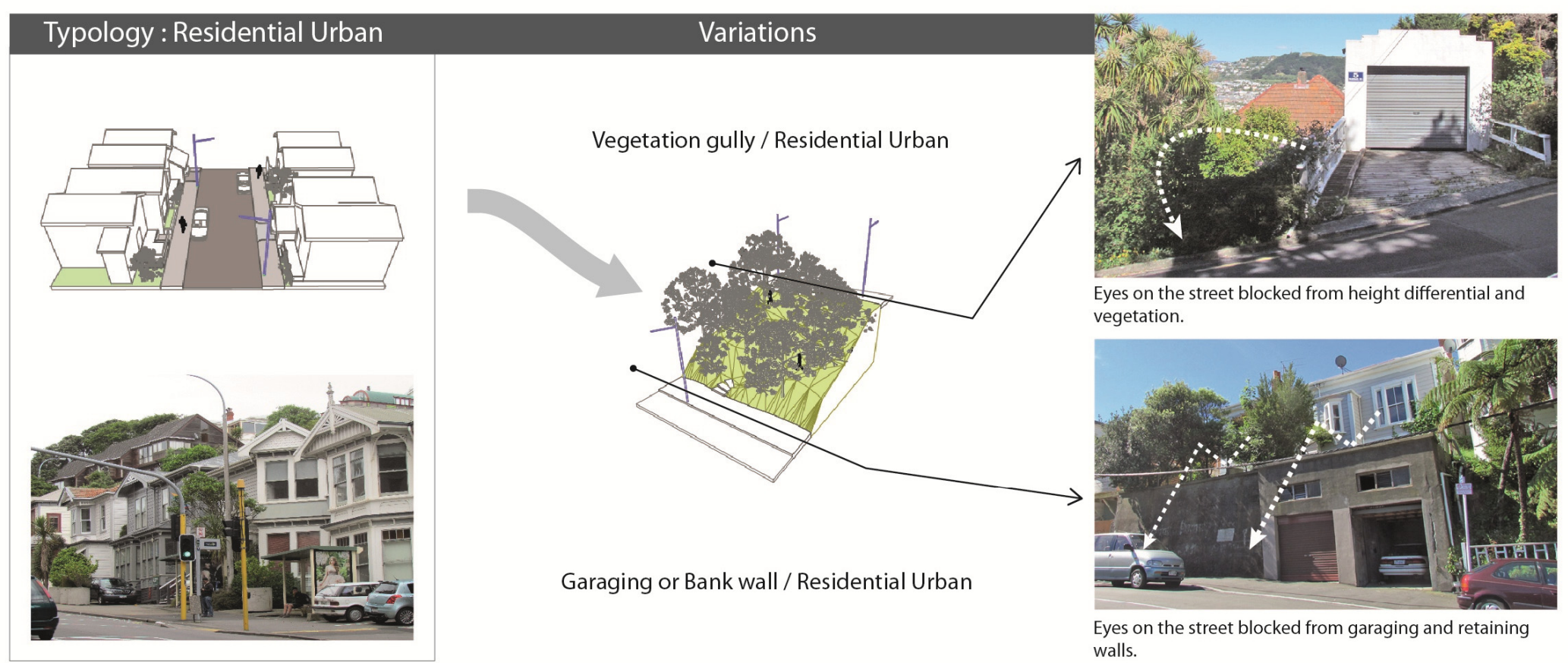

Figure 21: Some different ways in which 'eyes on the street' can be obstructed for a 'urban residential street' typology

\section{Example 2}

The second example is based on the shopping street typology. In a mixeduse building the ground floor might be leased by a retail shop, and have a number of residential floors above. However, in a different scenario a similar building, which has residential floors above ground floor, might have instead a restaurant on the ground floor. In both cases the buildings are categories as 'shopping street' typologies, yet both have completely different ground floor opening hours. This is problematic as accessibility, in terms of visual and social interest varies between each building at ground floor throughout the day.

The experiment found as a solution to these problems each typology needed to be broken down more specifically. So, for example, if a real 
world typology on site had a significant spatial condition on the ground floor that needed to be highlighted, the typology was labelled using this format.

Significant activity - ground floor / Typology - 1'st floor upward

\section{i.e.}

\section{Retail / High density apartment}

Or in other circumstances,

\section{Significant activity at the footpath's edge / Ground floor typology}

i.e.

Bank wall / Residential Urban

\section{Typologies and wider site context}

The second problem that emerged from this experiment was that the typologies and their simplified spatial conditions of the built environment were unable to consider the larger urban systems which the typologies function within. This became evident when the larger site conditions, which were influencing accessibility on site, went by unnoticed in the typological method analysis. For example, in many instances respondents identified a number of accessibility barriers to be present within certain 'real world' typologies on site. However, when each 'real world' typology was compared back to the initial typological study, the study proved incorrect as those 'real world' typologies were not supposed to cause any accessibility barriers. Conversely, at times in the experiment respondents viewed an entire street positively despite the street containing typologies that in theory should have caused an accessibility barrier. So why was this inconsistency occurring? And why was it proving difficult to find a definitive relationship/pattern between typological theory and the perceived accessibility barriers on site? I argue there are two reasons for this:

Firstly, accessibility within site is influenced by wider site conditions outside the context of the individual typologies. This was apparent in a number of situations during the cognitive surveys. For example, one of the respondents commented on their perception of safety at night when walking along a particular street (The Terrace) - The Terrace was categorised as an 'Urban Residential street typology'. According to the theoretical typological study, safety along the 'urban residential street' should be attributed to the surveillance from the after hour occupation of the houses. However, contrasting to this, during the cognitive mapping interviews the respondents attributed the feeling of safety in the evening along the street due to the high volume of vehicle traffic. The cause of this evening traffic is because The Terrace is as a principle road in the larger vehicle network, which feeds into a significant State Highway on-ramp 
(see Appendix D pg 224). Therefore, because the road network is a larger urban scale site condition that occurs beyond the influence of an 'urban residential Street' typology, however has still affected the respondent's perception of accessibility, this demonstrates that when researching accessibility within a site there are larger scale environmental factors that the typological method of assessment is unable to identify.

The experiment also highlighted that the respondents' perceptions of accessibility is influenced by spatial conditions simultaneously between typologies on both sides of the street, highlighting that it is important to think about a site being made up of more than just a series of individual typologies in isolation, but instead as a matrix of relationships between multiple typologies. For example, take the Bank wall (at ground floor) /

Urban residential typology and think about the spatial conditions that contribute towards the accessibility criteria of interest. As a theoretical typology in isolation, the level of built interest a pedestrian experiences in this spatial condition depends on the:

- $\quad$ Building's aesthetic, such as architectural details and material qualities of the bank wall,

- View shafts, whether the bank wall at ground floor still permits views to the building.

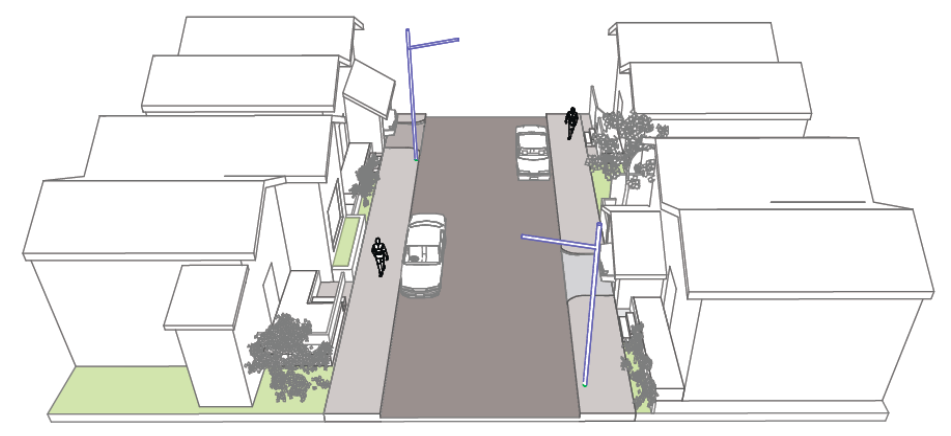

However, assessing built interest became more complicated in the design experiment. For example on a number of occasions streets that contained a run of 'bland' bank walls were not, surprisingly, considered by the respondents as being un-interesting, despite the bank walls failing to achieve either of the above two points. The reason for this was because directly opposite the road the pedestrians' interest was satisfied by a variety of interesting houses, as depicted in Figure 22. This example highlights that there is a tension in trying to understand the environment as a single simplified typological entity, when clearly pedestrian accessibility in the built environment is more complex. The environment is made up of many relationships between multiple typologies on either side of the road at a micro scale, as well as other larger macro scale influences. 


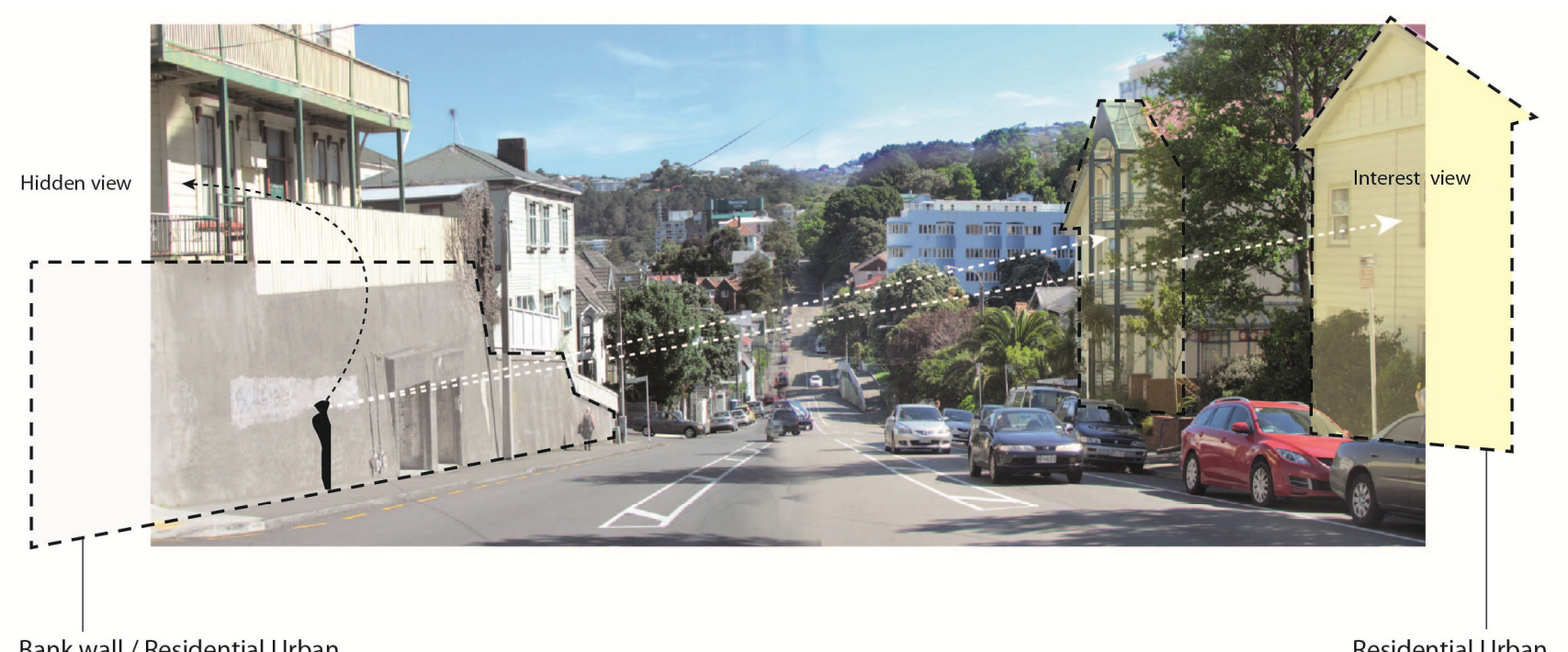

Figure 22: Diagram showing a person engaging with typologies on the adjacent footpath

\subsubsection{Further Direction for the Typological and}

\section{Cognitive Mapping Methodology of Site Research}

The final problem that emerged from this experiment was the difficultly of observing a pattern between the typologies within site, and the accessibility barriers raised by the respondents. Note this is a significant problem considering the intention of the typological method was to be a process for researching how pedestrian accessibility is affected by the design of the built environment. The absence of a clear relationship was due to two reasons:

- Firstly, the amount of survey information obtained for each area of site was insufficient. For example, when all the respondents' routes from the interviews were overlayed into a single image there were very few 'hot spots' where the respondent's routes overlapped. Therefore as a consequence a minimal range of perception data was gathered for each site. This is problematic because a large range of perception data must be obtained for 
each problem site/hotspot in order to truly investigate a relationship between the barriers and typologies.

- Secondly, the number of typological variations used in this experiment was too simplistic in comparison to the diverse range of real world typologies on site. As a result, failing to identify a pattern was therefore an indication that accessibility within a site is much more complex than can be explained through the typological analysis method used in the experiment.

Marrying a typological understanding of a site with the public's perceptions of accessibility is a somewhat disconnected process. On one hand, a user's perception of a site is unique and complex, affected by multiple typologies and spatial relationships between them, across a range of spatial scales. Yet in contrast, a typological method of analysis attempts to simplify the complex nature of site by removing and reducing these unique spatial qualities into a singular typology. As a result, this disconnection is problematic because it causes the typological method of analysis to produce an incomplete assessment of the site's built environment.

In conclusion, the hypothesis of this experiment was to uncover a pattern between the typologies and the accessibility barriers pedestrians perceived within a site. This experiment highlights however, that further research is required (in terms of a larger interview sample size and detailed typological study) before a pattern can be observed, and thus before a typological analysis can be used specifically to understand, and pre determine, where accessibility barriers are within a new site.

\section{Summary}

Limitations of the typology

The experiment found that using a typological method to carryout out site analysis is problematic. This is because typologies cannot identify where within a site, or explain how, pedestrian accessibility is affected by the built environment at a macro scale. This is significant because the highly important accessibility categories such as connectivity and journey proximity can only be improved through understanding the macro context.

The experiment identified the cause of this problem as being centred on the fundamental dichotomy between the complex nature of a site, versus the simplification process governing a typological method of analysis. For example, on one hand understanding the complexity of a site requires an awareness of the macro scale site systems that occur off the footpath; the diverse and unique range of spatial types that are present on a site; and, the interrelationships that exist between them. However in contrasts to 
this, the typology method of analysis, aims to simplify all these complexities to understand what is going on within a site in isolation.

Secondly, the experiment highlighted there is a disconnection between the simplified context-less 'theoretical typologies' (from the typological study), and the diverse contextual 'real world' typologies (from site). This was highlighted when the initial typological study proved unable to represent site in a typological medium, and thus made it difficult to observe a pattern between the typologies on site and the accessibility barriers that were identified in the surveys. The experiment highlighted that fundamental to this problem is that there is a unique contextual quality to each 'real world' typology in the built environment that is unable to be 'typologised' without an apparent endless number of typological variations.

Benefits of the Typology

Despite the earlier limitations, typologies are a useful tool for understanding in principle the spatial conditions of a site that are likely to affect pedestrian accessibility. Therefore, when typologies (which do not consider context) are used alongside the findings from the cognitive surveys (which are contextual), typologies can help researchers understand more about why the pedestrian accessibility barriers occur in the built environment.
In conclusion, when researching pedestrian accessibility barriers on a site in practice, there are a number of considerations that need to be made:

1. Professionals should have an awareness of the interrelationship between each of the real world typologies on site, and how they affect pedestrian accessibility.

2. Typological analysis needs to distinguish between:

- $\quad$ Theoretical typologies, which operate in a simplistic realm without context;

- $\quad$ Real world typologies, which are unique to context, and embody an accumulation of qualities that are inherent across a range of theoretical types.

3. Professionals should have an awareness of the larger systems on site that affect pedestrian accessibility and that occur beyond the influence of the typologies. This could range from the influence of the typologies present across the road, to a macro scale system such as the location of the site within the city's transport network. 


\subsection{Further Direction: The 'New}

\section{Pedestrian Design Strategy'.}

This chapter found a number of limitations with the initial 'Hypothesis Pedestrian Design Methodology'. Thus in reaction to these limitations, over the course of the next three design experiments a new 'Refined Pedestrian Design Strategy' was developed. The first iteration to this new methodology is diagrammed opposite.

4. Locate the access typologies on site in order to understand the spatial principles that are causing each significant barrier. Therefore becoming familiar with the level of resources required to resolve the barrier.

3.

Conduct analysis of the greater urban

context in order to see which accessibility

barriers and place-making opportunities,

which were identified through the

cognitive surveys, are of most importance

to improving the larger pedestrian and

public space network.

Ne

What are long-term changes that need to be made within the larger urban context in order to improve: connectivity of the

pedestrian network, as well as the quality

of public space network, at a macro scale?

2.

Conduct a citywide survey using the

cognitive interview method, to highlight

the 'hot spots' where accessibility barriers

are present in the city's pedestrian net-

work. In particular between the residential

catchments and the CBD.

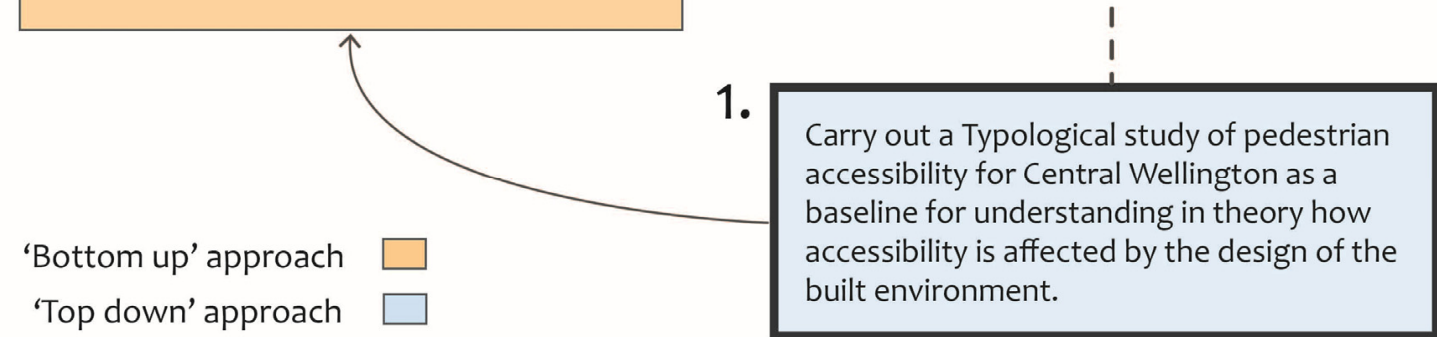


The previous chapter trialled the first two stages of the initial 'Hypothesis Pedestrian Design Strategy'. These were a typological analysis of pedestrian accessibility in Central Wellington as well a pilot study of the cognitive survey method. The purpose of this next chapter is to explore the remaining stages (3-7) of the hypothesis methodology with the main objective of discussing:

\section{$?$}

How can pedestrian barriers in the built

environment be resolved most cost efficiently

through design? What are the consequences

that arise from a focus on efficiency, and how

do they need to be addressed?

Chapter 5: Pedestrian infrastructural solutions

The chapter is structured in the following order:

Design Experiment 2

The experiment investigates using 'standardised typological solutions' as a way of resolving pedestrian accessibility barriers within the built environment. The experiment uncovers: firstly, the pros (such as cost efficiency) and cons (the lack of response to context) of using this design approach. Secondly, that making small interventions on the macro scale 
can be a catalyst for larger scale change, altering how people move through the city can affect the way a the city functions.

\section{Design Experiment 3}

The experiment begins by addressing from 'Design Experiment 2' the key problems that arise when using a standardised typological design, particularly when the design interventions fail to respond to the surrounding context. Following this, 'Design Experiment 3' uncovers that there is an opportunity to improve the quality of public space within site during the process of resolving accessibility barriers. The experiment pursues this opportunity by identifying the areas of site that have placemaking potential and subsequently designing to develop these areas into new public space.

\section{Design Experiment 4}

Lastly, 'Design Experiment 4' combines what was learnt in the previous three design tests, Design Experiment 1, 2 and 3, into one complete strategy for research, designing, and implementing pedestrian infrastructure. Most importantly, this experiment demonstrates that designing for accessibility barriers, and place-making opportunities, on a micro scale has a significant effect on the larger macro scale. However, only if both the micro and macro scale are researched, designed, and implemented, for together.
5.1 Design Experiment 2: 'Creating Typological

Solutions'

The purpose of 'Design Experiment 2' was to explore two things:

- Firstly, to trial the cognitive mapping survey technique on a larger sample size.

- Secondly, to test how effective a standardised typological approach could be at resolving accessibility barriers on site in a limited economic funding context.

A standardised typological approach was used because of two reasons:

- The initial typological study simplifies site into categories that are characterised by a common spatial condition, thus it is an easy progression to design standardised solutions for each typology.

- Standardised solutions are both time and cost efficient because the design can be replicated on multiple sites, which enables parts of the design to be mass produced.

Note, the Adshel Bus Shelter (Figure 23), is an example of a standardised solution that is replicated throughout Wellington's built environment. 


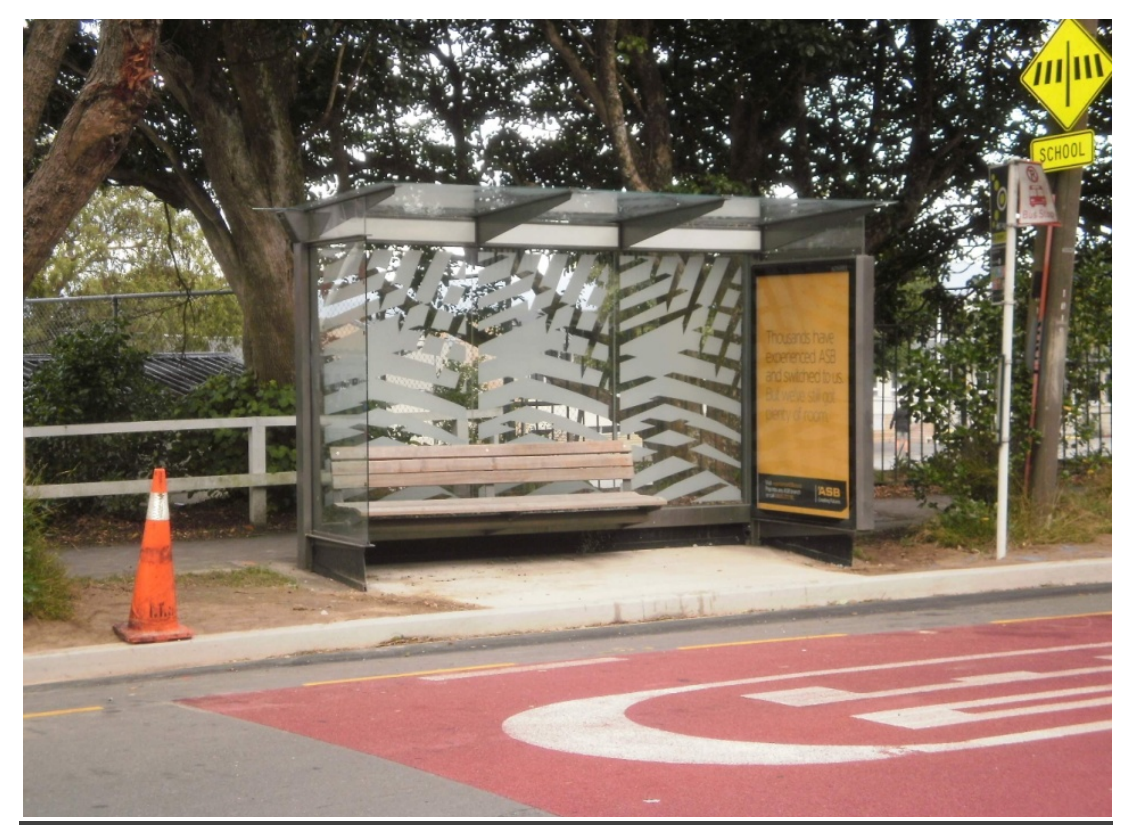

Figure 23: Adshel's standardised bus shelter

\subsubsection{Method}

The main design experiment was carried out using the same 'barrier hot spot' site identified in the cognitive survey in 'Design Experiment 1' (Figure 19 pg 70). Note prior to this, a pilot test was carried out using a separate site; however the findings from this will be discussed later on after the main design experiment.
To begin with the Salamanca hot spot was broken down typologically (Figure 24) and compared against the qualitative comments from the interviews (Figure 25). The diagram shows the barriers present along Salamanca Rd were:

- Comfort: because of an "absence of shelter from weather"

- CPTED lighting safety: because of some areas being "too dark".

- Route proximity: because one respondent had to walk an "unnecessarily long route".

- CPTED social safety: because "not enough people were around"

Re-representing site in a typological manner was important as it allowed each typology to be compared against the accessibility barriers that were raised by the respondents. Then by using the initial typological study to understand how each accessibility barrier is created by each of the typologies, the following process was carried out:

1. Typological solutions were created to address each of the accessibility barriers on site.

2. The typological solutions were documented and expanded further into a 'design guideline' format, so that the solutions could be transferable to other sites later on.

3. Each typological solution was then applied to site.

The following Figures 25 - 32 demonstrate this process. 

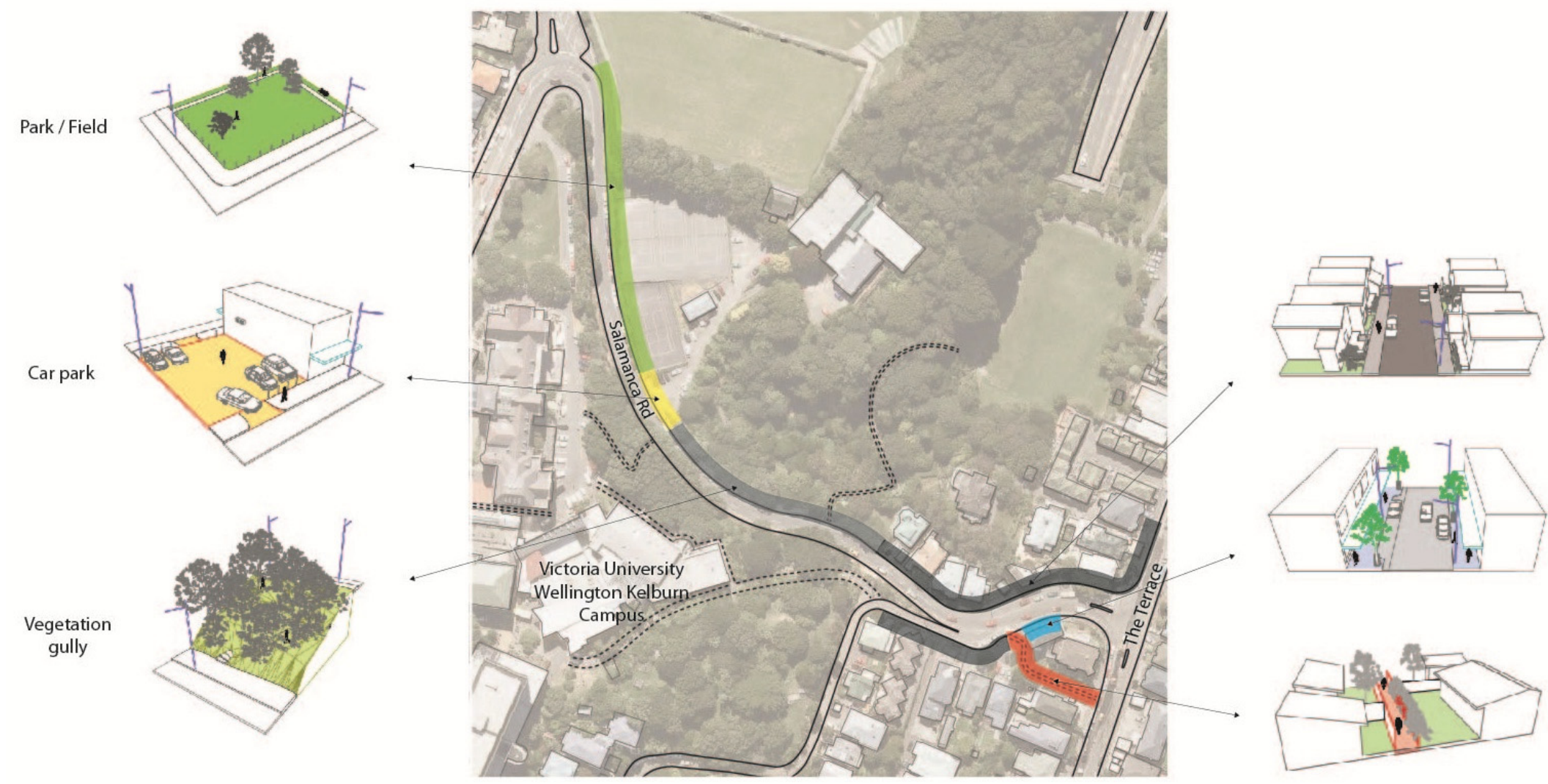

Urban residential

Car park

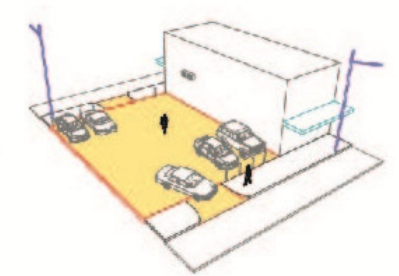

Vegetation
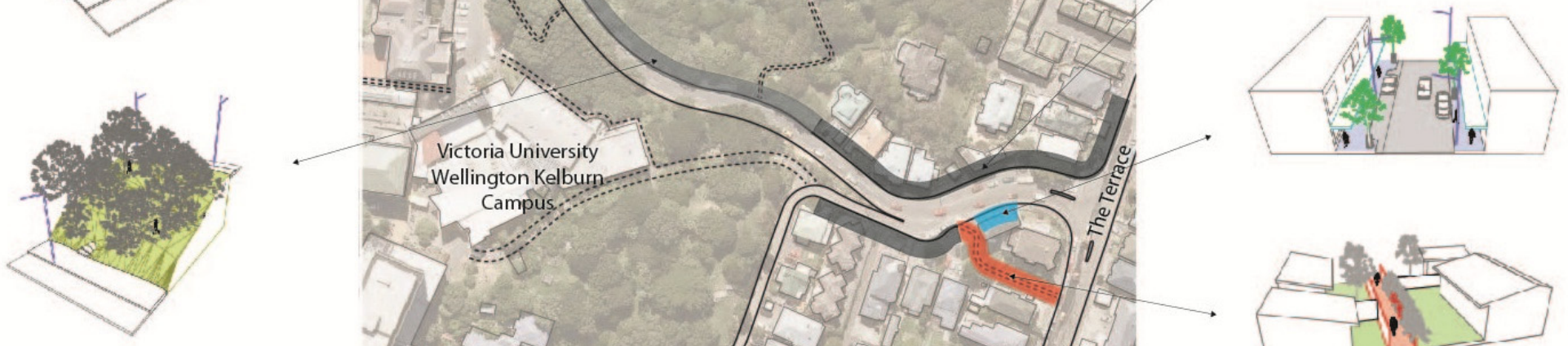

Retail / Hospitality

shopping st

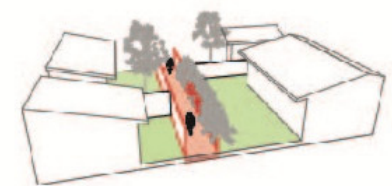

Residential Passageway

Figure 24: Typologies within barrier 'hot spot' site 


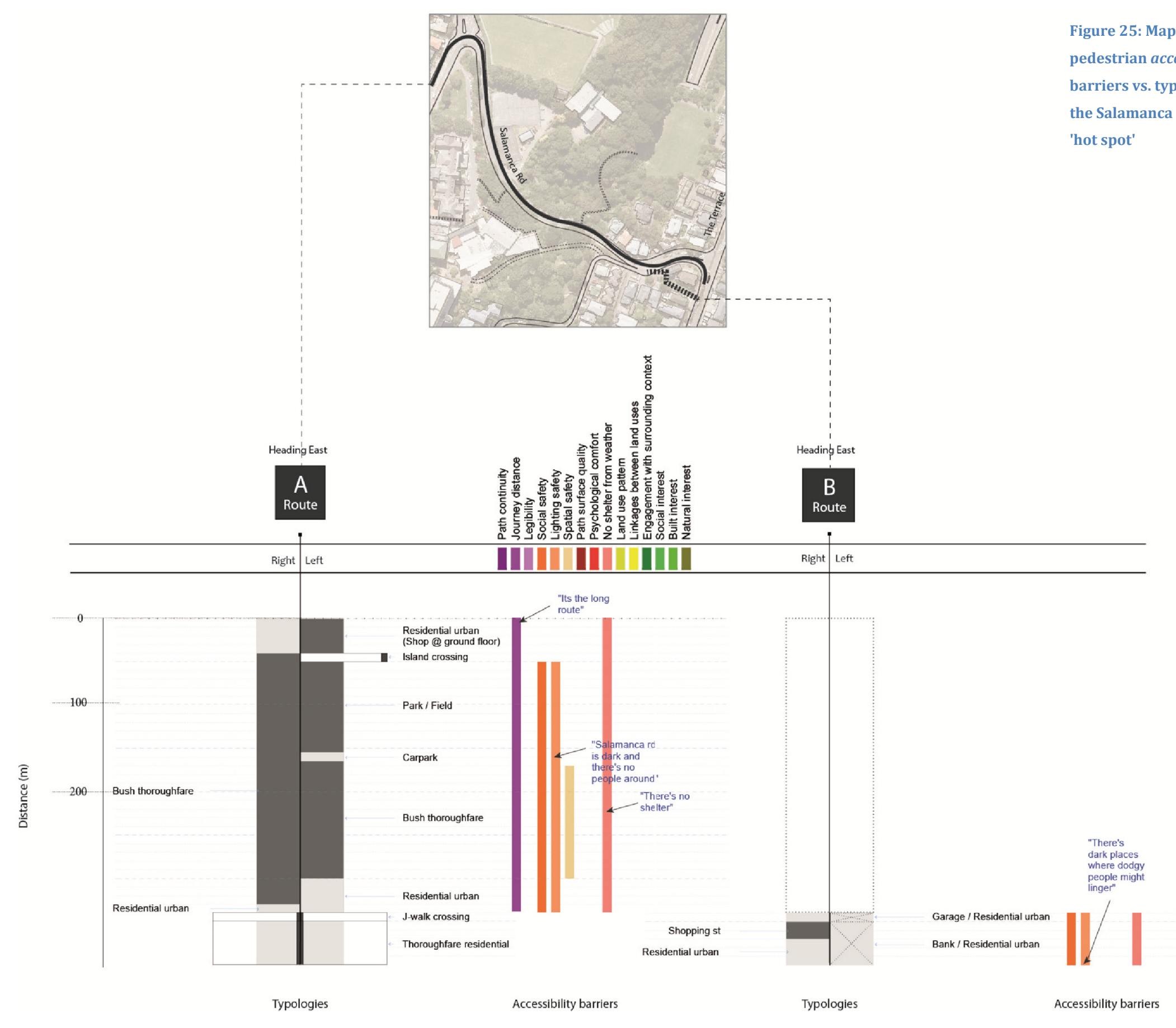




\section{Example 1}

The cognitive surveys identified four accessibility barriers at the area of site shown in Figure 26. This area of site was overlooking an 'urban residential St' typology. The barriers were:

1. No shelter from weather.

2. Absence of evening lighting.

3. Journey distance (as it was not the shortest route for some respondents)

4. The lack of social safety in the evening.

Note only barriers 1 and 2 can be addressed through a typological intervention as their sphere of influence is refined to the micro street scale. Thus barriers 3 and 4 were not addressed in this experiment as they required interventions to be made in the wider macro scale environment which is beyond the influence of a typological solution. 
Type $=$ Street front urban residential

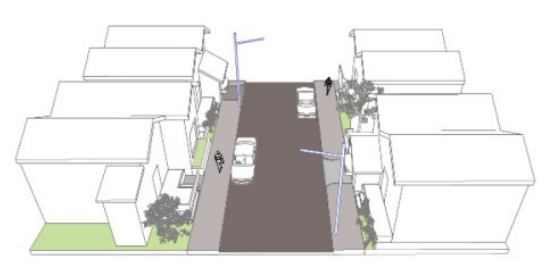

Figure 26: Land use

typology and associated

accessibility barriers

identified on site

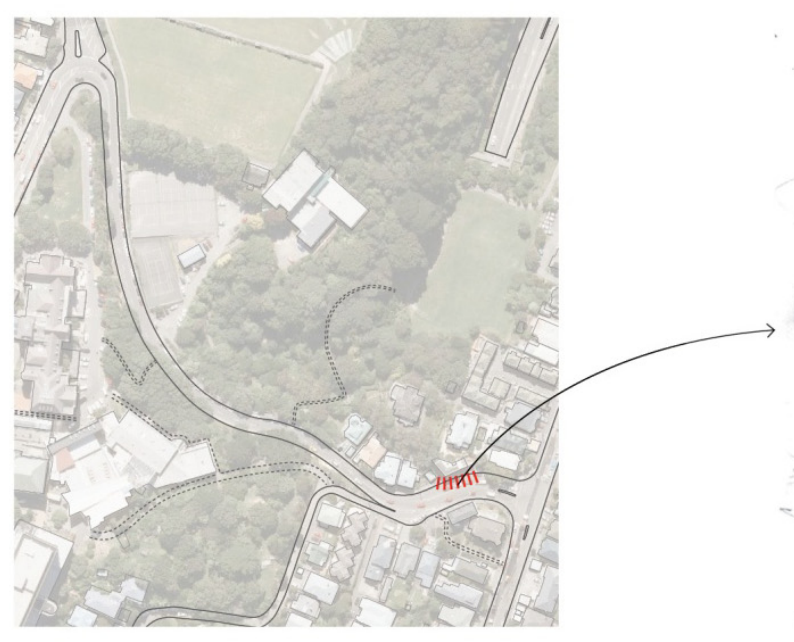

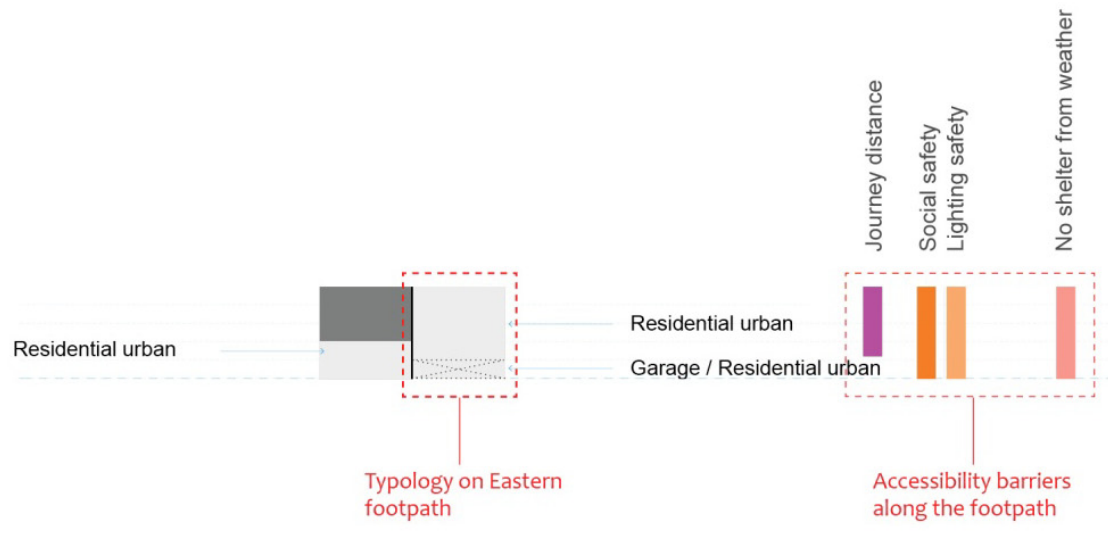

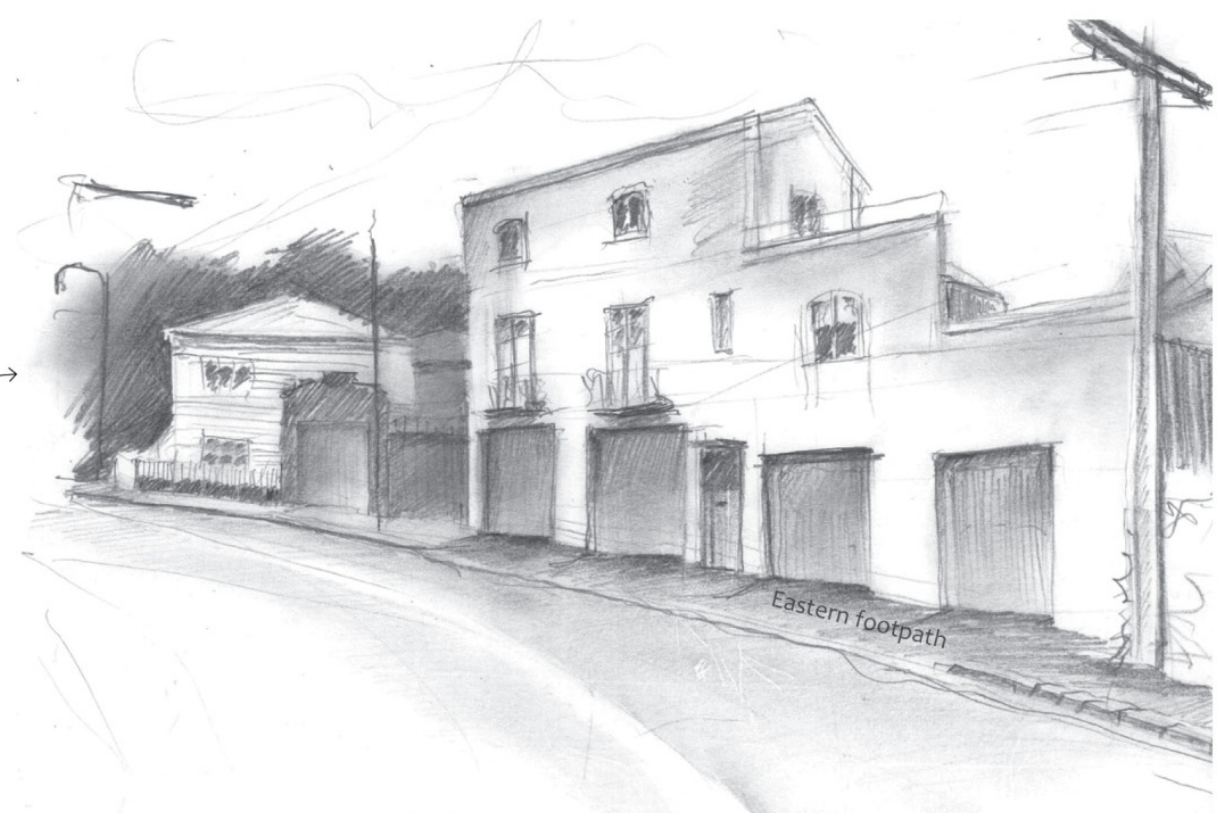


Figure 27: Standardised typological canopy shelter solution for the 'urban residential St' typology.

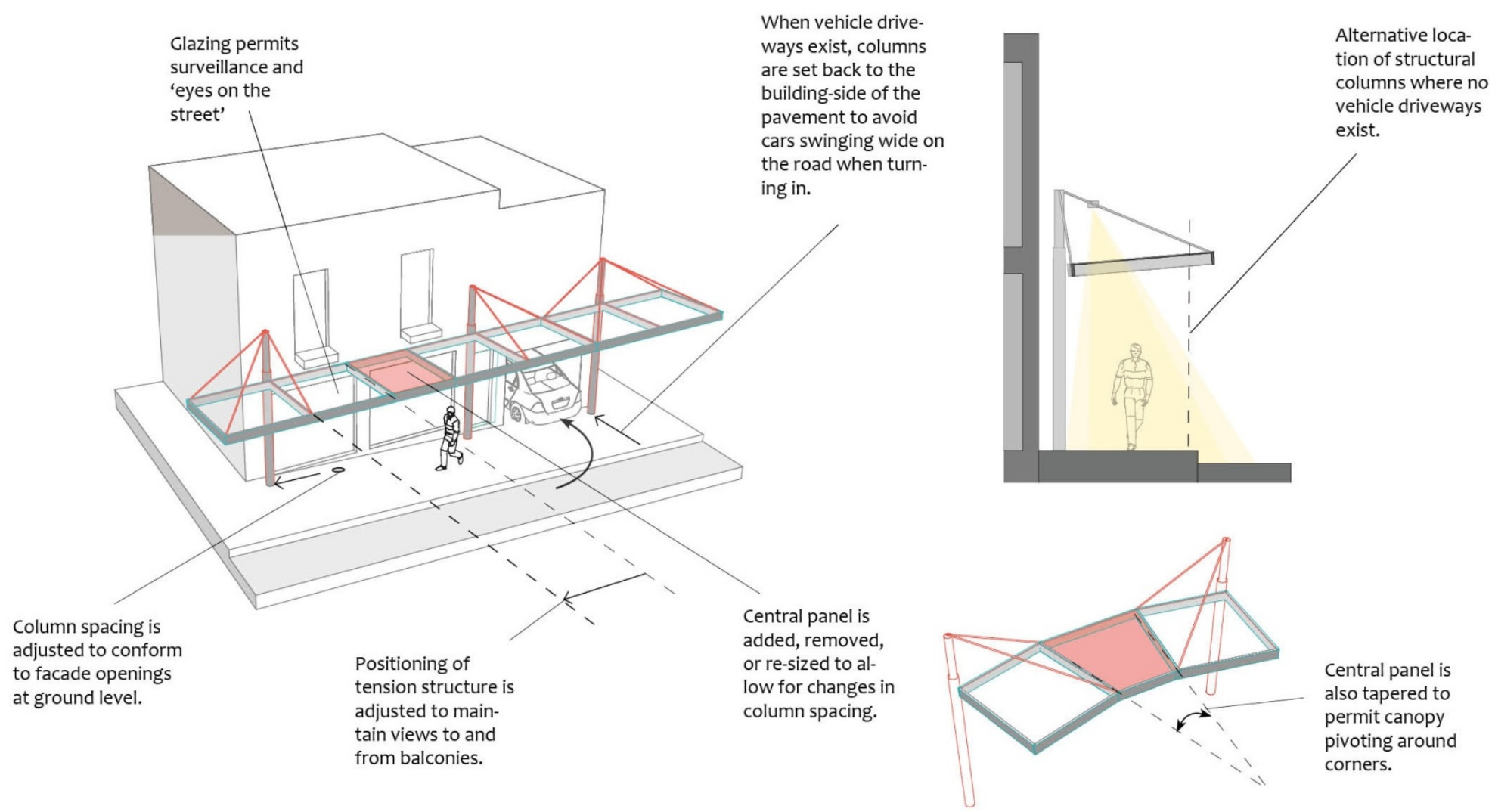


Figure 28: Standardised typological canopy

solution for 'bank wall'

typology

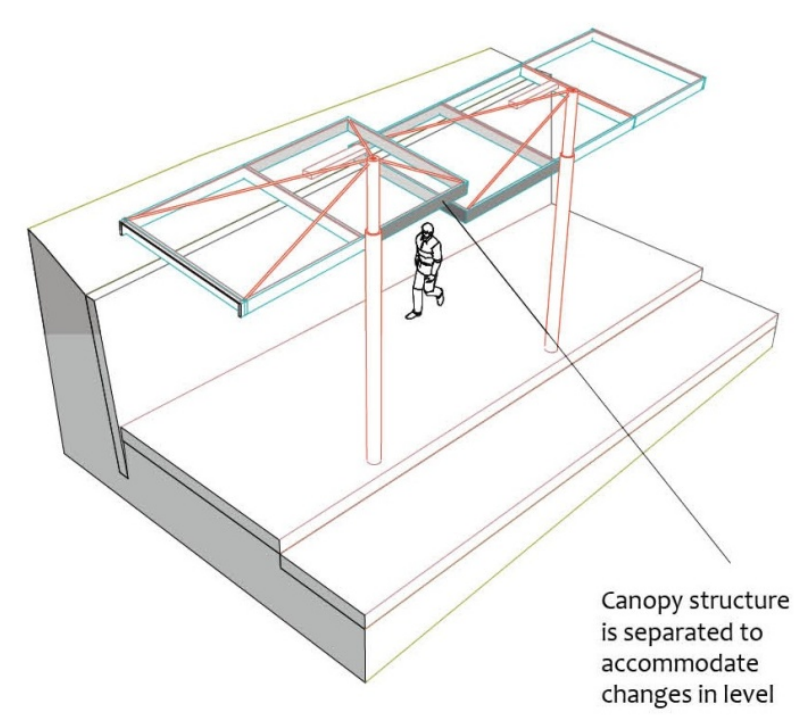

Lighting from above

projects down

through glass panes

illuminating the foot-

path, and also pre-

sents an opportunity

for interesting visual

graphic projection.

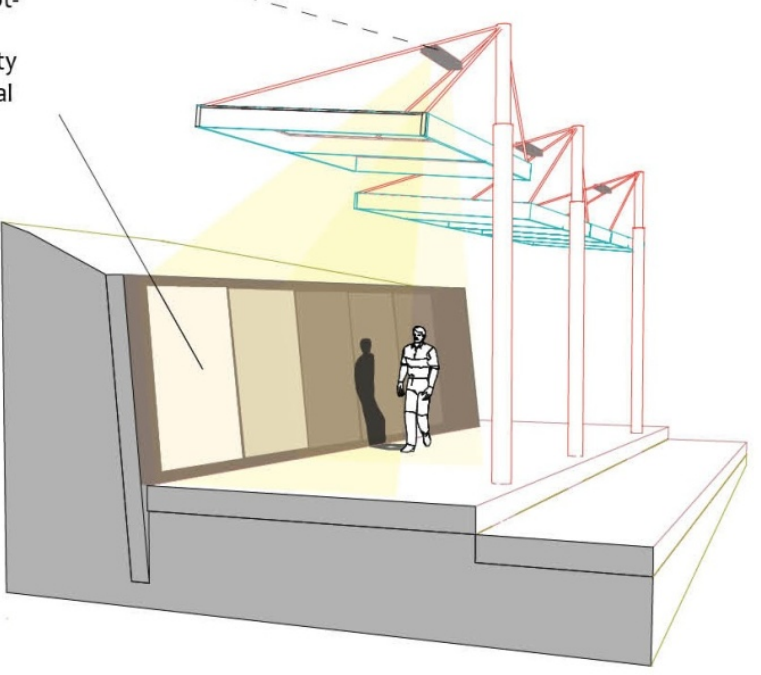

accommodate
Columns casted

into bank

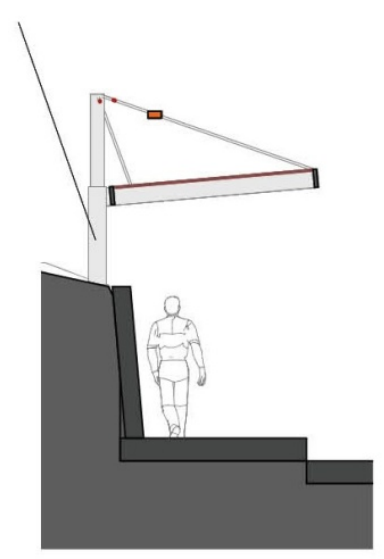

Low Bank Wall

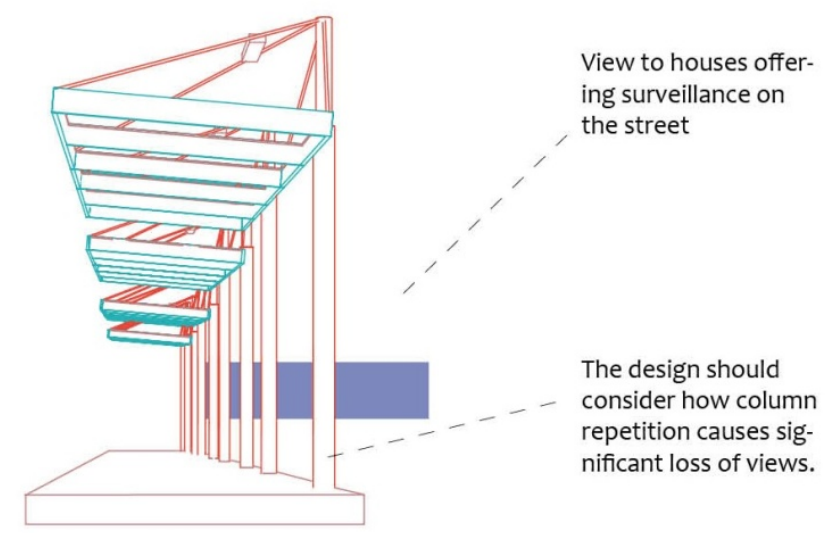



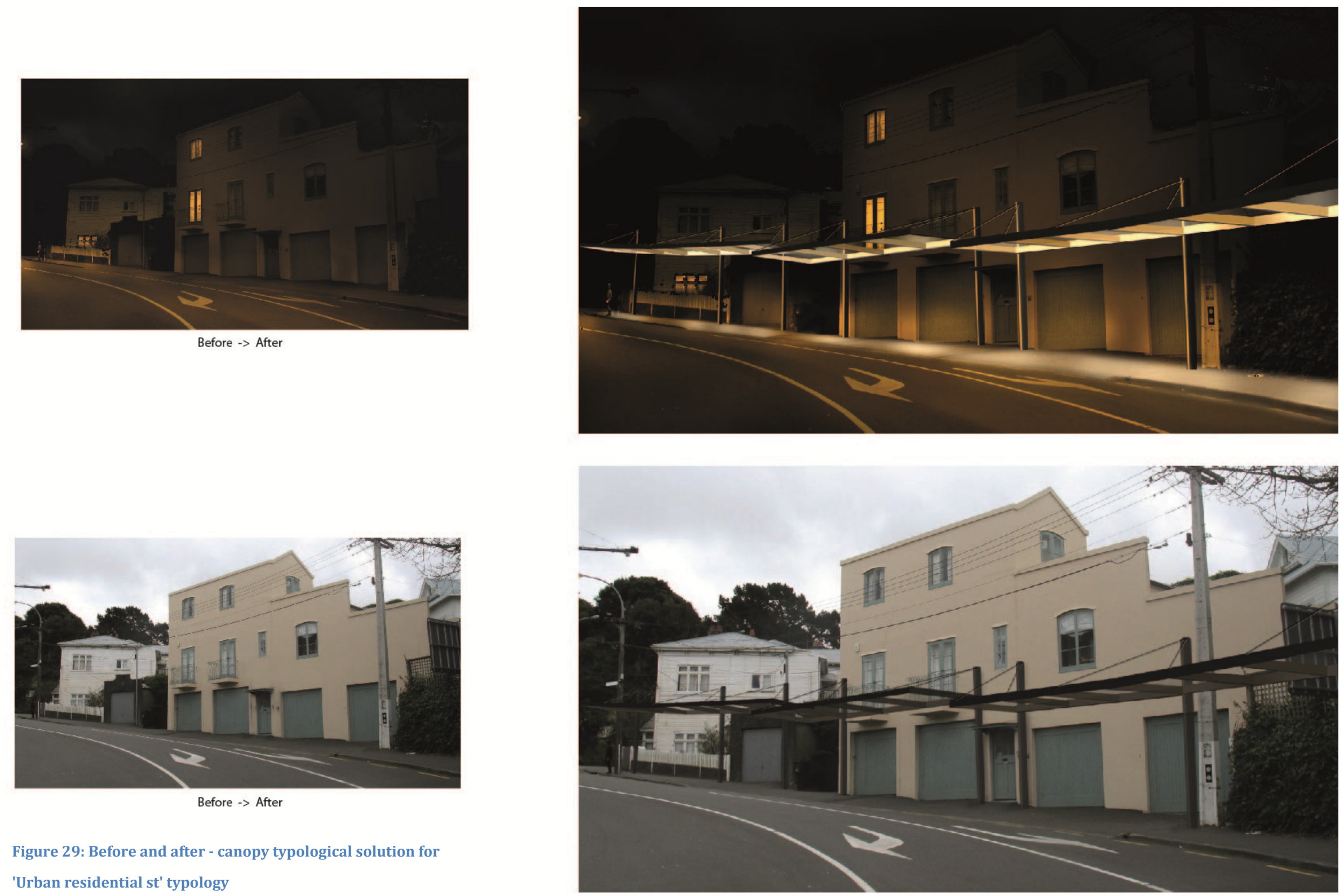

Figure 29: Before and after - canopy typological solution for 'Urban residential st' typology 


\section{Example 2}

The cognitive surveys identified the five accessibility barriers along an area of site shown in (Figure 30). This area of site was categorised as a 'vegetation/gully' typology (Appendix A pg

212). The barriers were:

1. Journey distance

2. Social safety (lack of people around in the evening)

3. Lack of sufficient lighting

4. Spatial safety (CPTED) due to an adjoining path that leads directly down a bank into secluded dense vegetation

5. No shelter from weather

Note as with the previous example, barriers 1 and 2 were not addressed as they required intervention on a wider scale than what could be resolved through a typological intervention at street level. Barriers 3 - 5 however, could be resolved using the typological intervention. 
Type $=$ Vegetation

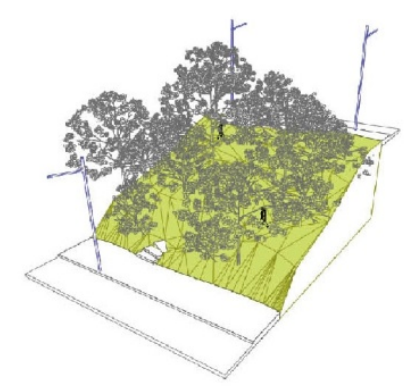

Figure 30: land use

typology and associated accessibility barriers

identified on site
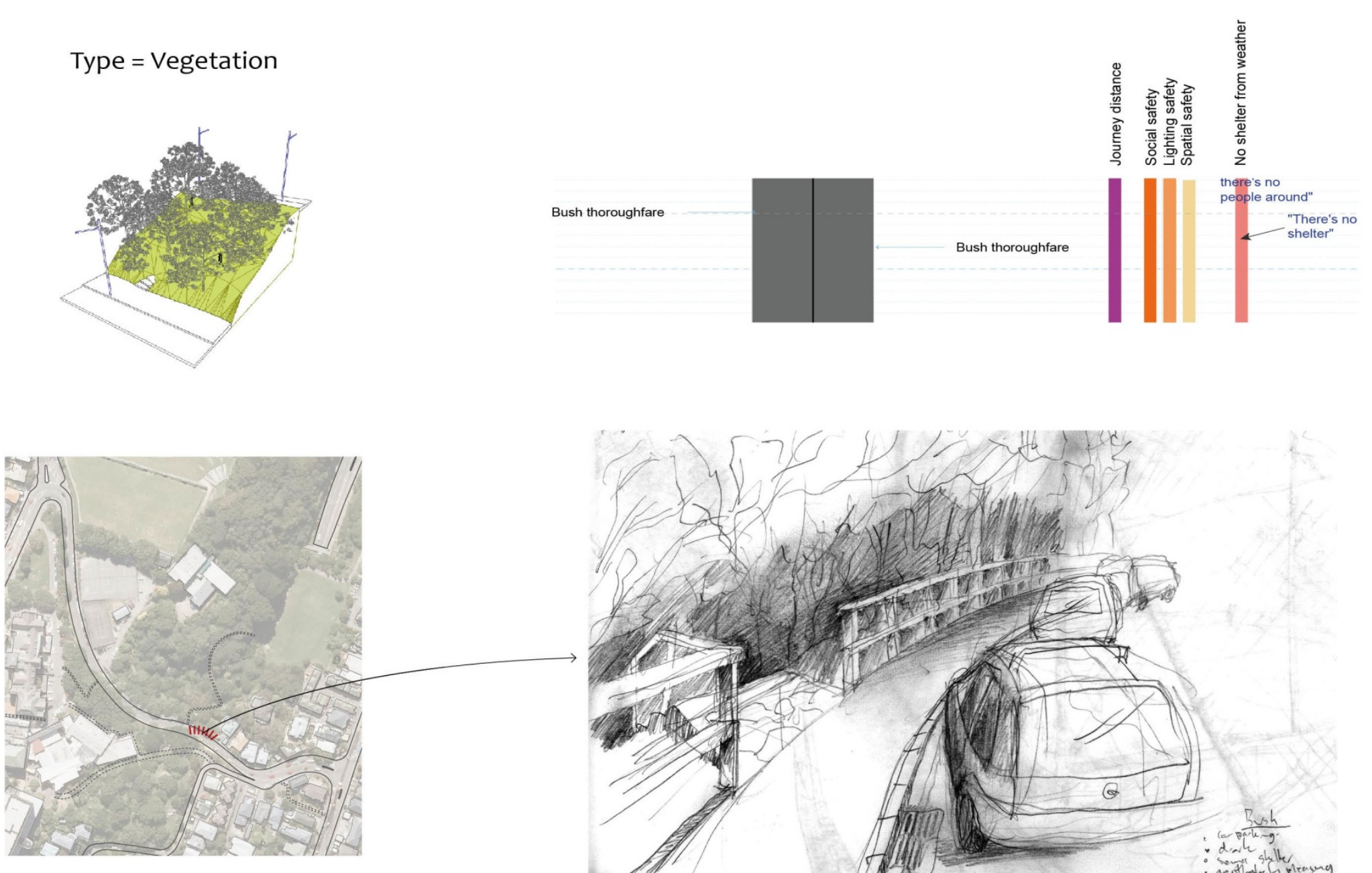

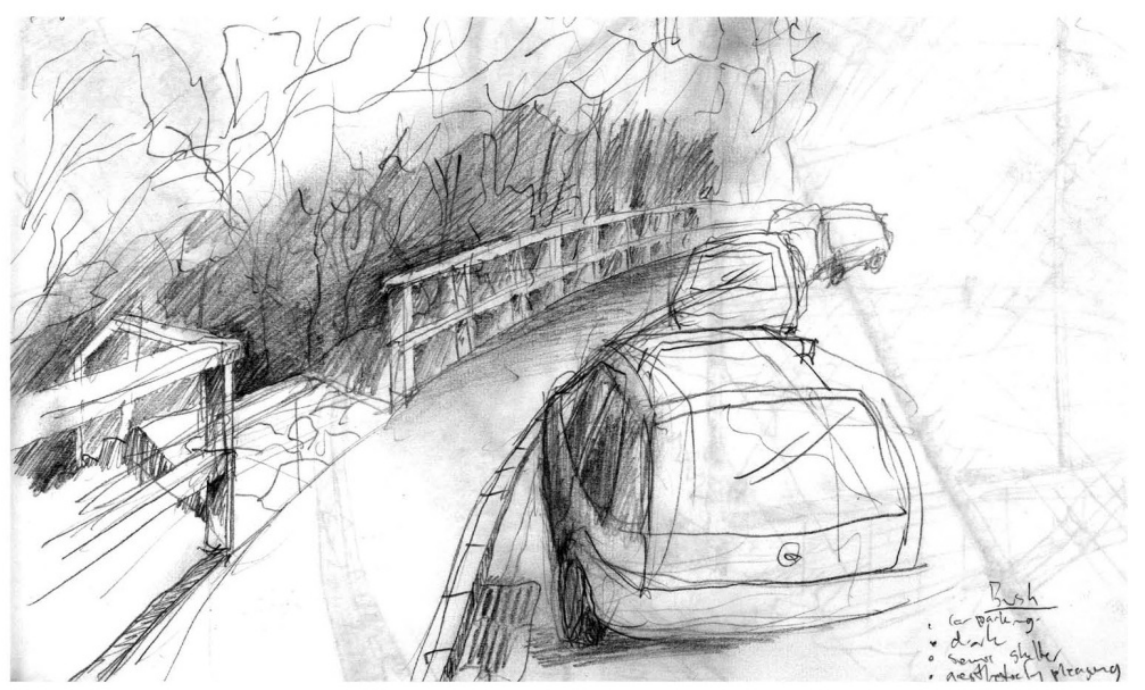


Figure 31: Standardised

typological shelter solution

Standardised typological solution for buffering between path and dense vegetation (CPTED)

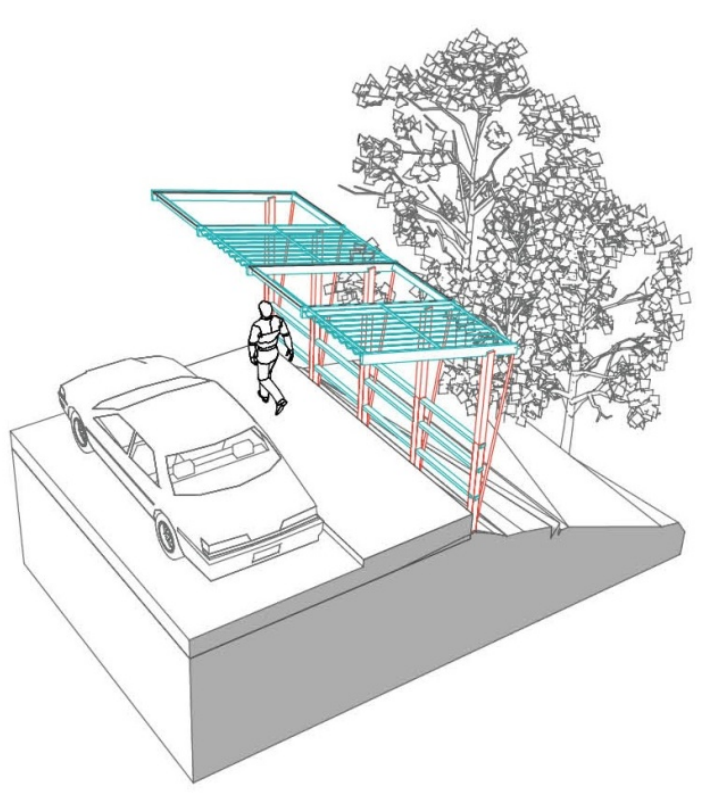
pitch adjusted for site wind intensity When roadside parking exists and the footpath width is limited, to avoid collisions with manoeu-

vring cars columns are

removed along the roa

side edge of the foot Canopy

path.

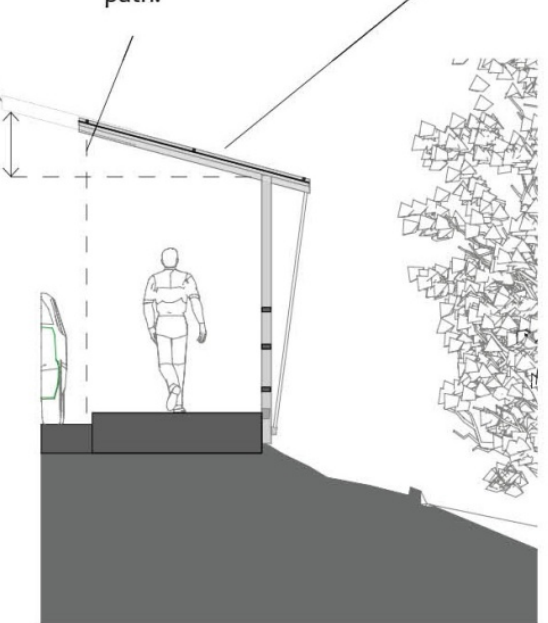

105

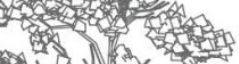

4. 13

a 1 .

H 3
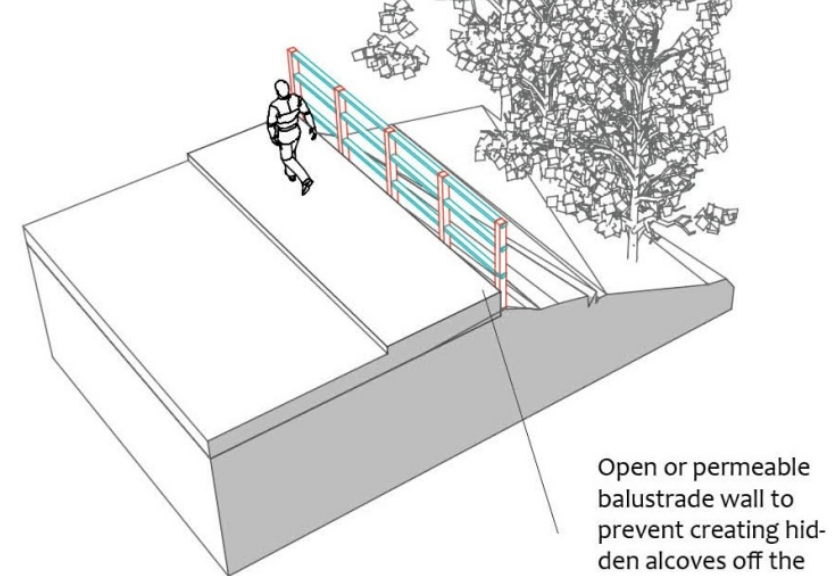

prevent creating hicfootpath from path edge
Flood lighting

into vegetation to lighten dark

Introduction

of a balustrade

provieds a safety

barrier

Safety buffer created by clearing

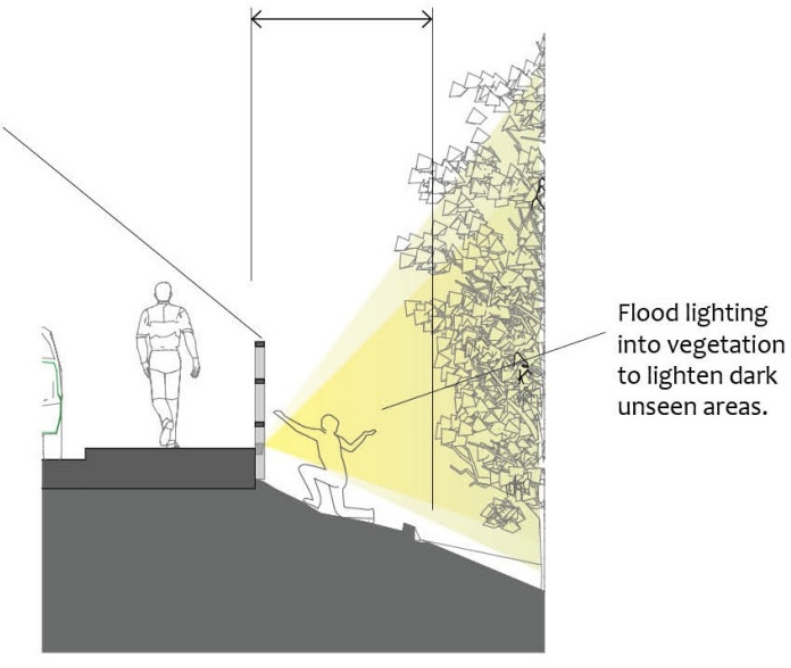


Figure 32: Standardised

(CPTED) typological solution

for the junction where a side

path meets the footpath

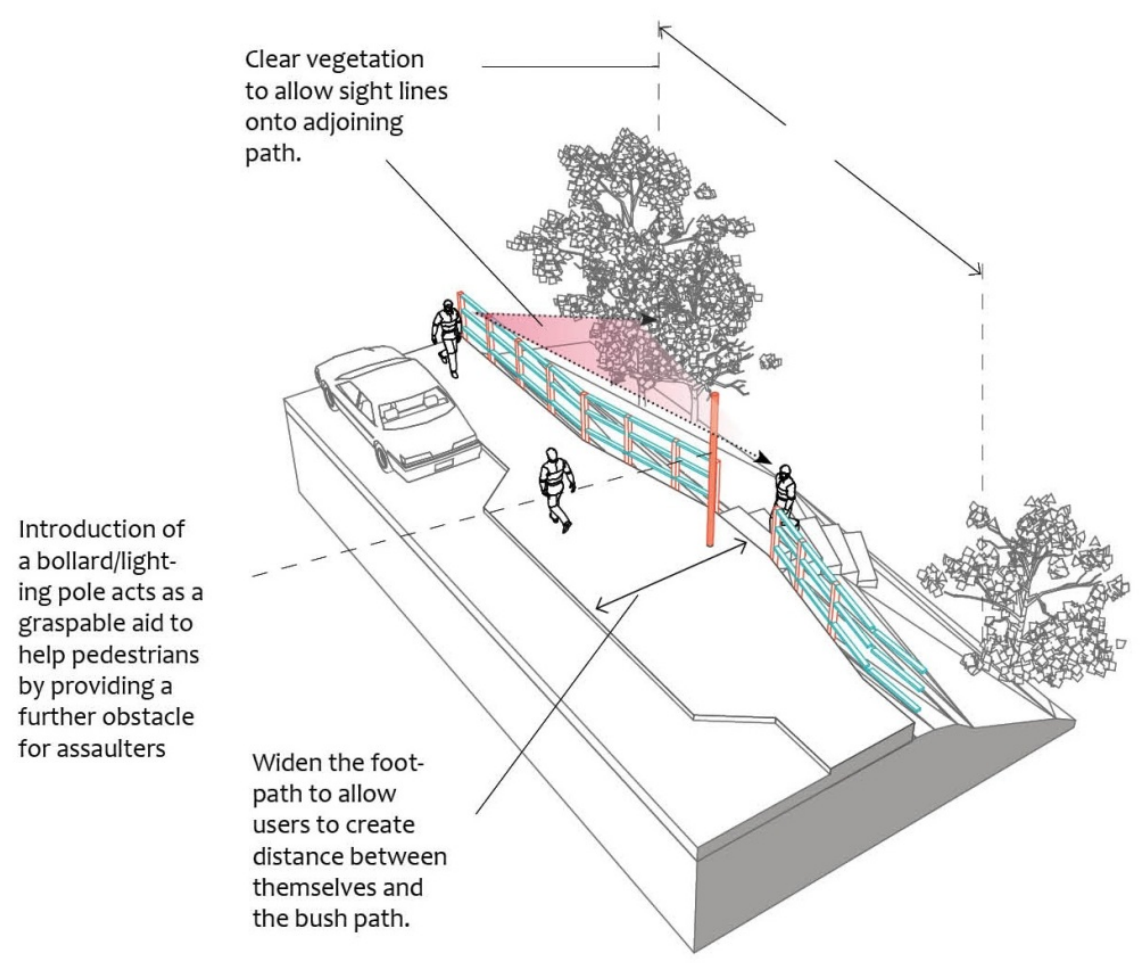

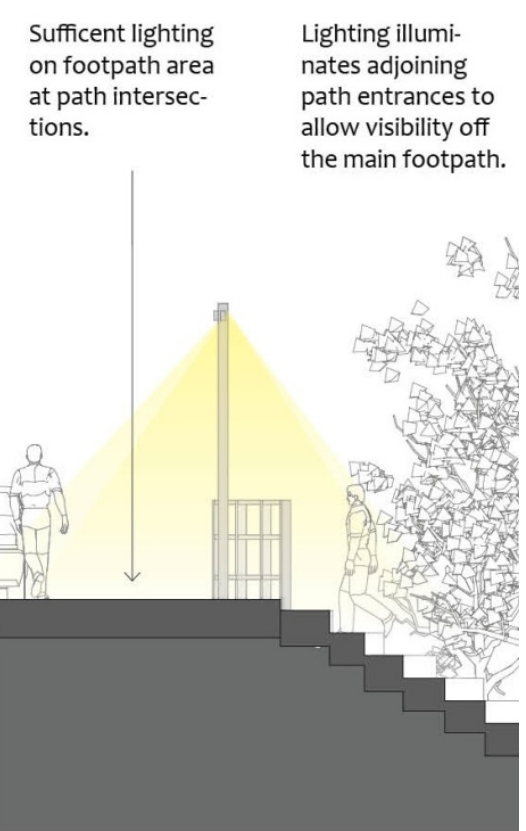



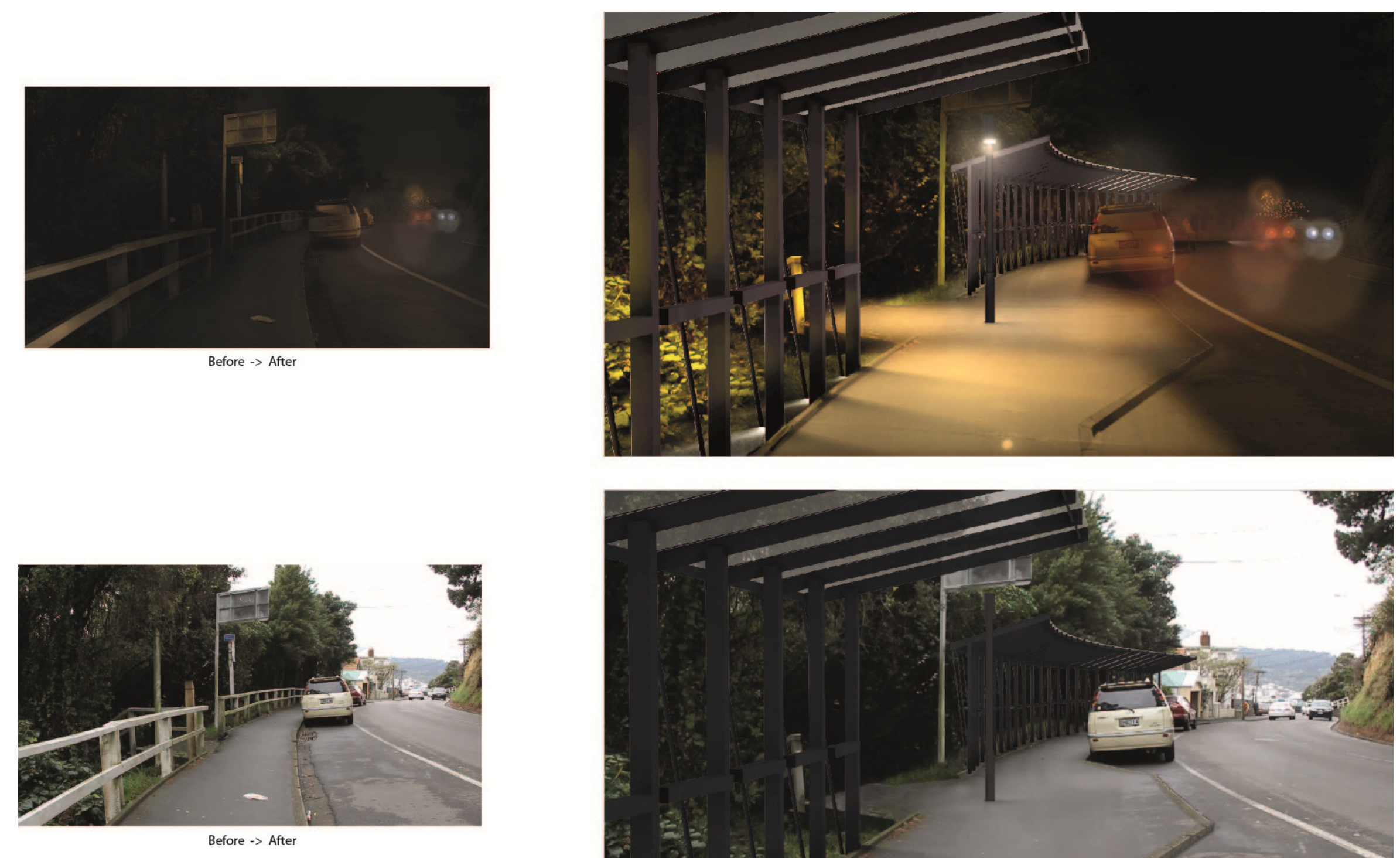

Figure 33: Before and after - canopy typological solution for 'vegetation' typology

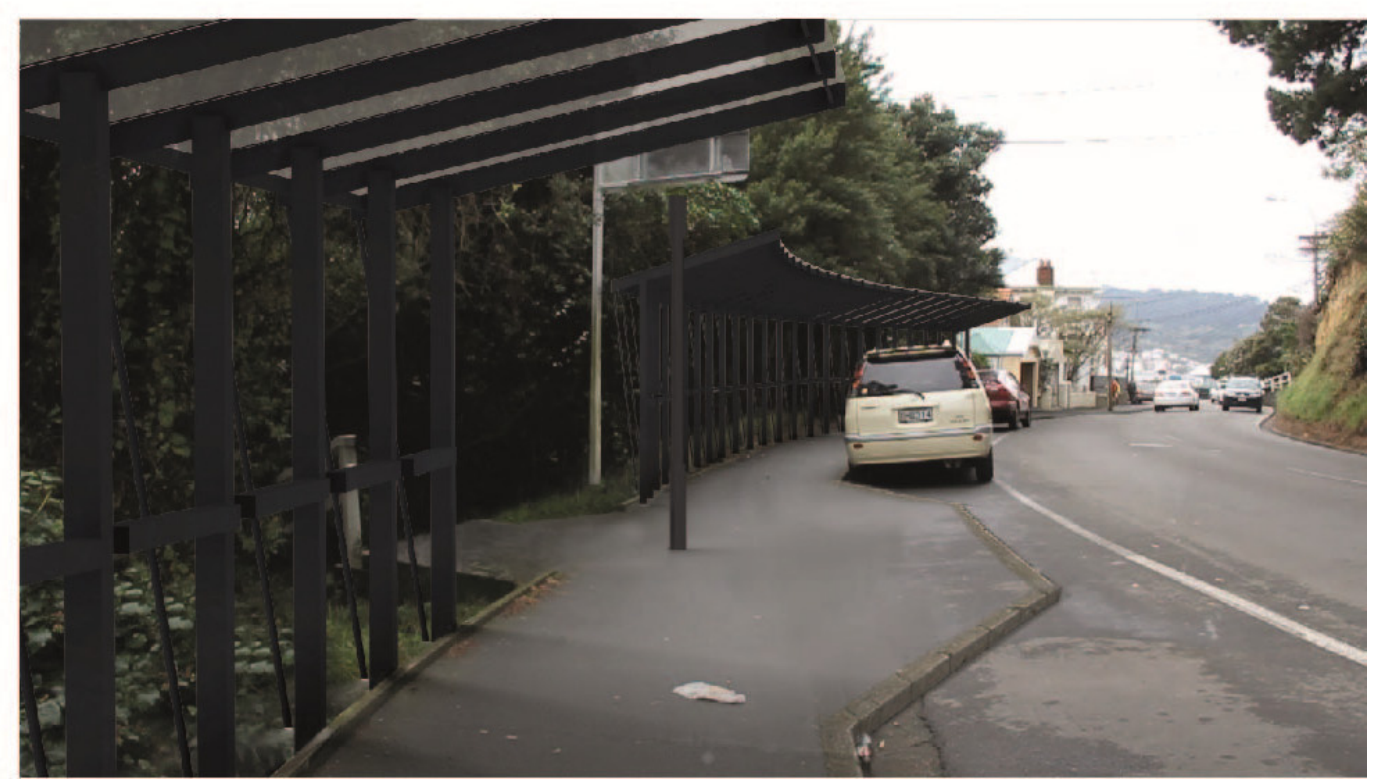




\subsubsection{Findings}

The discussion will begin with the findings from an earlier pilot test, which tested what problems would arise when employing this typological solution method of design.

\section{Pilot test}

When designing a solution to the accessibility barriers that were identified in the cognitive survey, the pilot test found that the designer needs to be aware of the other typologies within site and not just the location of the accessibility barrier. This is because by being familiar with the other typologies within site, the designer may be able to find other routes through site that could resolve the accessibility barriers yet require less intervention to do so. For example, when resolving the respondents' accessibility barriers, a variety of solutions were possible when other routes were considered that were different to the routes identified in the cognitive survey. In one interview, a respondent identified:

- Firstly a lack of shelter along their route,

- Secondly, a concern for safety due to the regular presence of people whom lingered on the footpath outside a large state housing complex.

When addressing this problem a dilemma was presented between the two possible solutions:
On one hand, a sensitive solution to the 'concern for safety' would be to provide two path options to allow pedestrians to walk past the state housing complex at a distance. However, in order to provide 'shelter from weather' along this alternative route, this scenario resulted in two problems.

- $\quad$ Firstly, placing a canopy shelter in front of a visually appealing historic church (Figure 34).

- Secondly, this canopy would be an intervention that requires some level of sophistication and customisation in its design in order to avoid blocking views to the church, and clashing aesthetically.

Conversely to this, in an alternative scenario, which on one hand would preserve the views and heritage quality of the historic Church by diverting the shelter intervention across the road, would however not eliminate public interactions outside the state housing complex (Figure 34). The benefit of this second option is that the blank wall typology along the opposite footpath requires much less design attention. Also the blank wall is suited to a 'standardised typological solution' that results in a much lower design and build cost than the alternative customised church canopy.

This dilemma reinforces that using a 'top down' typological study in addition to the 'bottom up' qualitative cognitive survey is important. This is because although the cognitive surveys can specifically locate the 
accessibility barriers present along a respondent's route, the pilot survey demonstrated that the design solution might end up shifting away from the original surveyed route. The problem therefore, is there is no survey data for the new route, and as a result no knowledge of what barriers need to be addressed by the designer. This thesis argues that if the designer uses a 'top down' typological study to become familiar with the typologies along new and unfamiliar routes through a site, the designer can use the typological study to learn about what accessibility barriers need to be addressed in these areas. 
Lingering people

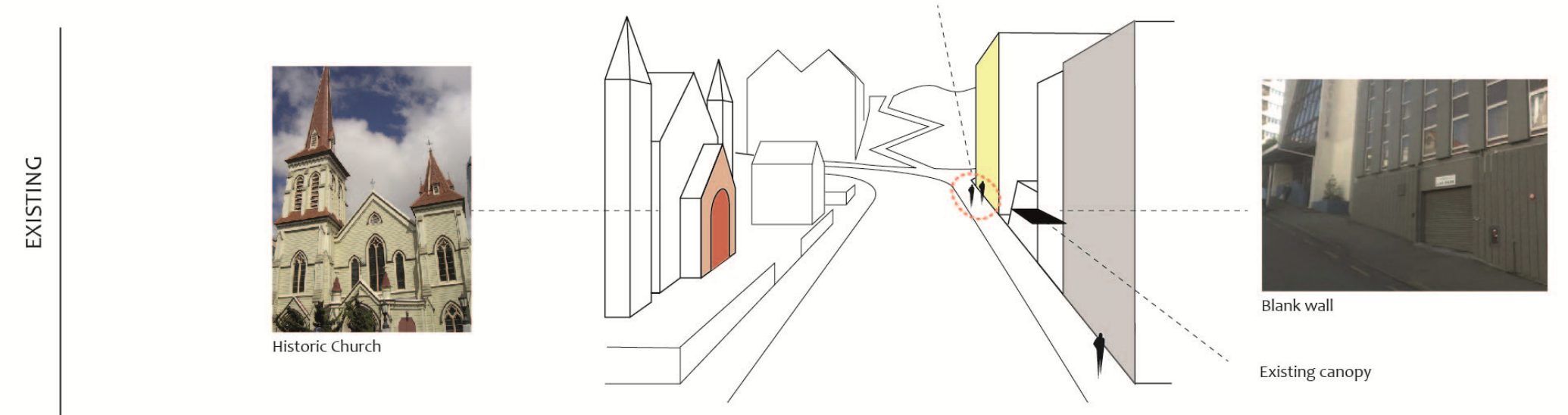

Path option (A)

Path option (B)

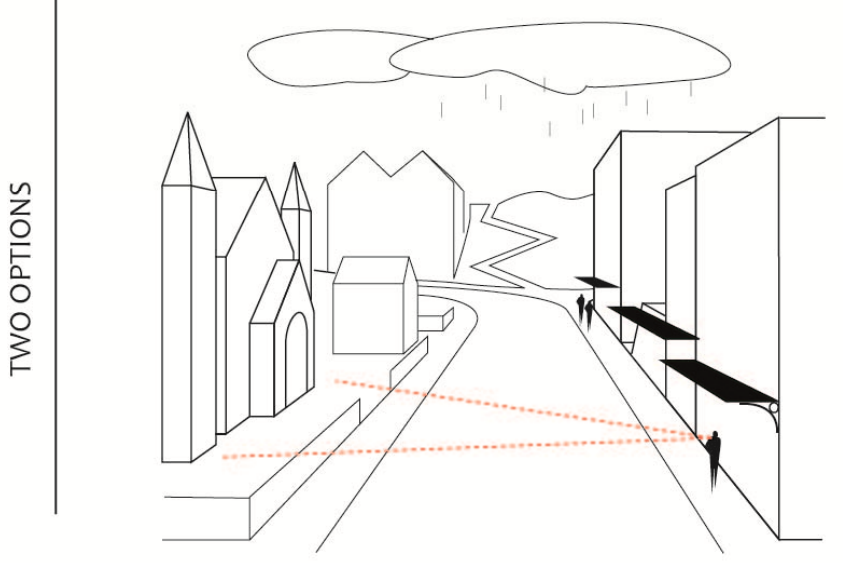

Maintained view to Historic Church

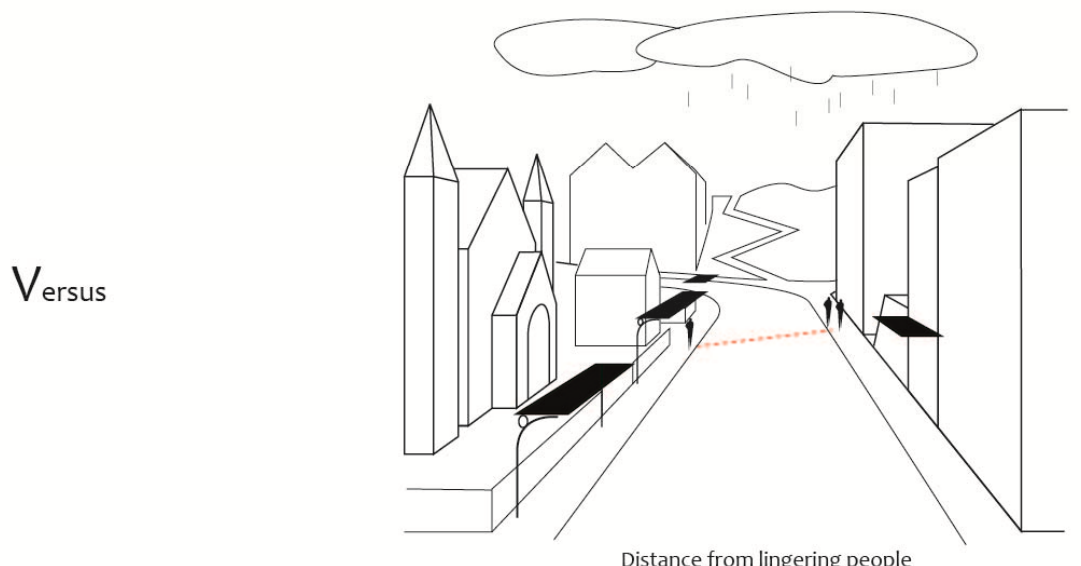

Distance from lingering people

Figure 34: Diagram showing two different options for introducing shelter along a street 


\section{Plug in typological solutions}

In addition to the findings from the pilot test, 'Design Experiment 2' uncovered two limitations of using typological solutions to resolve accessibility barriers within site. These limitations were:

1. Typologies have no awareness of the site's context and do not consider the surrounding environment.

2. When using a typological method of designing the process fails to recognise the hidden place-making qualities within site and subsequently opportunities for improving the quality of public space in the pedestrian network are lost.

1. Typologies and their lack of response to the surrounding context Firstly, at the micro scale (the street), the typological solutions failed to respond aesthetically and formally to the surrounding context. The design experiment demonstrated that through using a standardised typological design process, designing for materiality and form became arbitrary, as the final outcomes ignored the unique characteristics of the surroundings. This was problematic, as responding to the contextual qualities of site affected how the design solution fitted into its surroundings. This is clearly evident in the standardised canopy shelter solution designs, Figure 29 and 33 earlier. Therefore despite the fact that standardised designs were able to resolve the problem accessibility barrier, the designs were unsuccessful in their response to the adjacent buildings or vegetation.
The second example of the typologies failing to consider the surrounding context is highlighted through the key simplification ideology behind the typological process. The experiment demonstrated there are two contrasting ideas behind a design process that uses standardised typological solutions (as a means of cost efficiency), and also customised solutions (as a means of achieving responsiveness to a site).

On one hand there are the simplified spatial principles of a typology that operate without context. These are the spatial principles behind, safety from vehicles, path ergonomics, shelter from weather etc, which are theoretical principles that can be understood in a diagrammatictypological manner. Because they are in a diagrammatic form they can be easily developed into a standardised typological solution. For example Figure 31 demonstrates the spatial principles for achieving a safe sheltered path alongside a 'vegetation bank typology'.

However in opposition to this, there are contextual qualities such as character, materiality, and 'sense of place', that occur because of a site's relationship to its surroundings over time. These spatial qualities are unique to each site and therefore cannot be simplified, explained, or categorised into standardised typological principles. When converting site into a typological medium, the spatial qualities that are unique to each site are filtered out immediately, and this causes standardised typological solutions to be un-responsive to the surrounding context. 
Potential for existing

turning bay to become

pedestrian/shared zone.

Thus increasing the

Bus stop

amount of public space

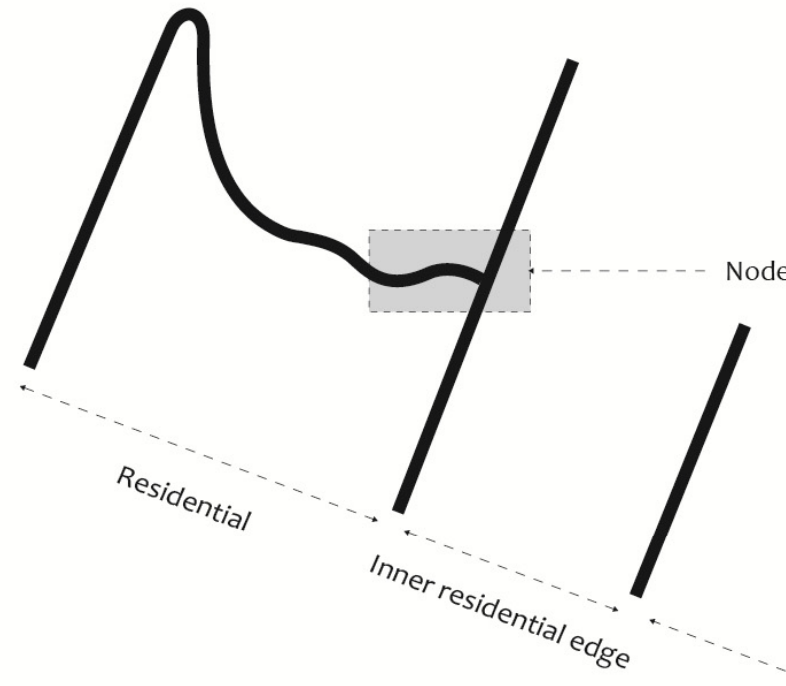

$C B D$

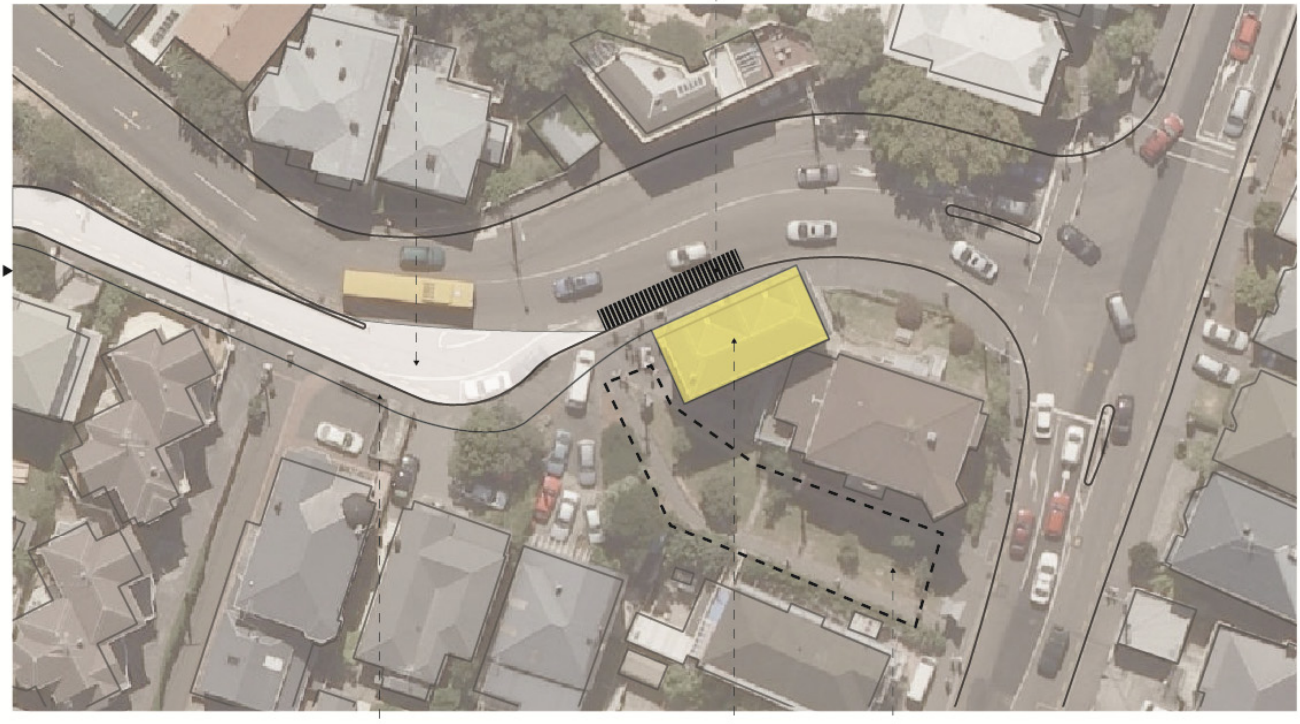

Existing footpath

Dairy Green space 
The Lost Opportunity for Releasing the Untapped Potential of Site

In addition to typologies being un-related to context, the experiment revealed that opportunities to improve the quality of public space in the pedestrian network are lost when using a typological approach to design.

Throughout the experiment it was revealed that the designer's mind is constantly active; instinctively searching for 'low hanging fruit' within the surroundings that have potential to be transformed into something fantastic if given the necessary attention. As a result the following exciting observations were noticed on site irrespective of the typological method of analysis:

- $\quad$ Figure 35 was an area identified at the Eastern end of site as having potential to be developed into a new public space area. This was because the area was located at the CBD end on Salamanca $\mathrm{Rd}$, which, is along one of the main pedestrian routes from Victoria University to the CBD; has a dairy, small park area, bus stop; and space for the footpath to be widened. These site factors offer a diverse range of activities, and spatial variety to this area, and thus justify why it would be appropriate to develop it into a new public space.

- In a second example (Figure 36), an upper car park area that overlooked Salamanca Rd was also observed as having potential to become new public space, and also to provide a new access way up to the University. The site was significant for two reasons:
Firstly due to its location on a key edge that currently provided under-utilised access between the iconic University Hunter building and Salamanca Rd - the primary commuting corridor

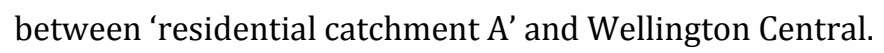

Secondly, improving the existing run-down pedestrian connection that passed through the site would increase the number of pedestrian routes up to the university. This would allow the University to present a new face to the city, and subsequently could re activate this edge by drawing commuters travelling up from the CBD to the newly developed pathway.

At this point I came to the realisation that through analysing something as simple as how pedestrians move through the environment, a designer can achieve two things. Firstly not only can they influence pedestrians' perceptions towards walking, by relieving the most intense moments of accessibility barriers in the city. But secondly, through small interventions such as re-developing a run-down pathway, have the ability to be a catalyst for larger change within the urban environment by influencing the way the pedestrian network functions. With this realisation in mind, throughout the experiment it proved difficult to ignore these opportunities simply because they were not observed through the earlier typological method of analysis. This presented a problem, as these exciting opportunities could not be realised through using a standardised 

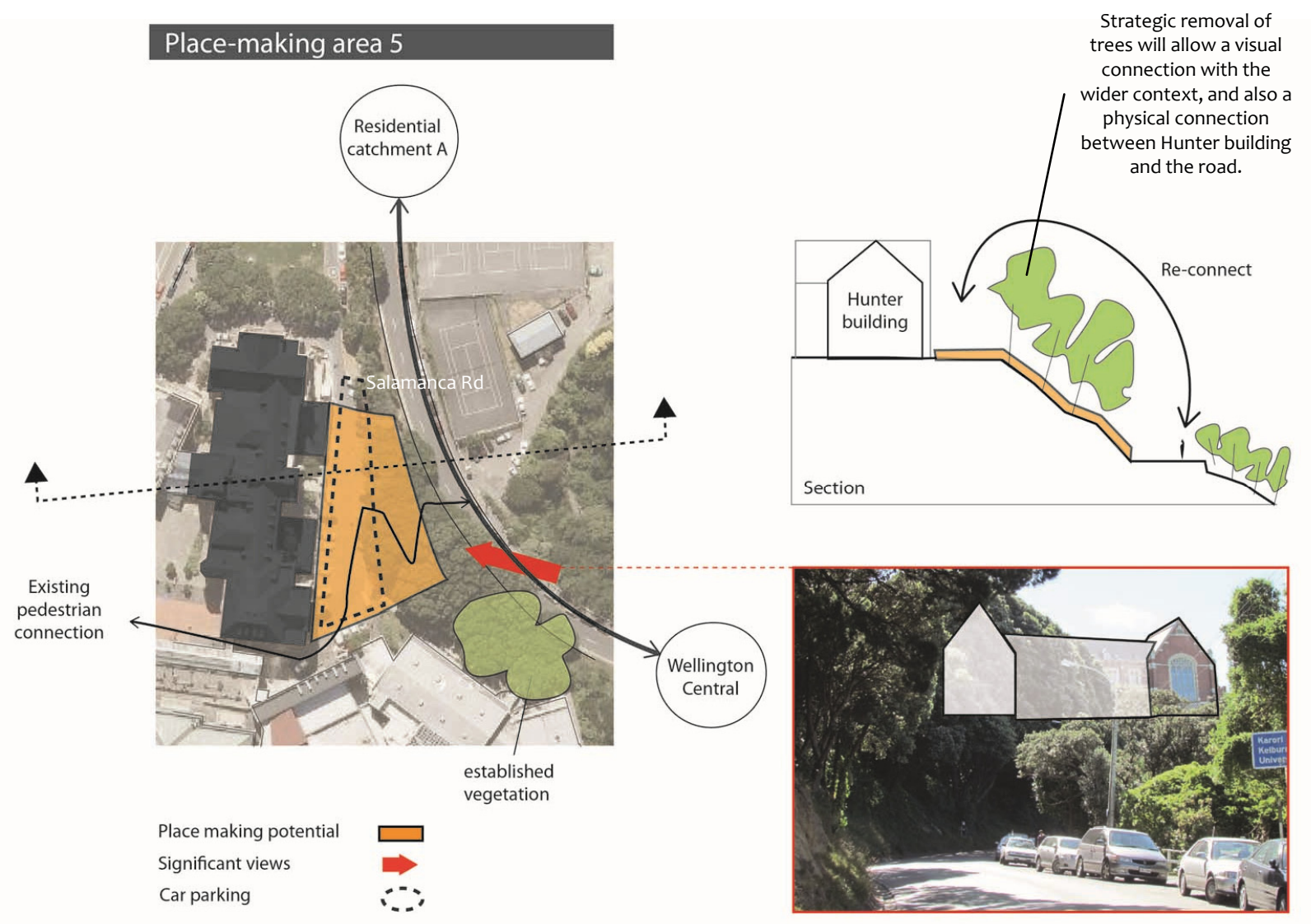

Figure 36: Qualities of the car park site that mediates between the University and Salamanca Rd

typological solution as each opportunity required a uniquely customised response. Therefore a dilemma arises here between resolving pedestrian barriers cost efficiently, using a standardised typological solution, which results in no aesthetic response to context or capitalisation on the unrealised place-making potential in site; versus improving pedestrian accessibility through a customised response, which can unlock the hidden potential of a site and potentially influence something greater in the larger context. In reality the latter would make for better practice as the designer has an opportunity to improve the quality of a site in many ways.

In summary these criticisms highlight, firstly, that using a standardised typological method of designing in isolation is problematic when it is used 
on sites that have significant potential. And secondly, that there are other considerations within a site besides pedestrian accessibility barriers that need to be accounted for when designing.

The next section will discuss the writings about 'place and place-making' by Peter Downton and Nick Beattie. Their writings are important as they outline the role of the designer in creating successful places, and best summarise the problems that this experiment has encountered in a search for a typological solution method.

\subsubsection{Further direction: The Untapped Potential of Place}

The previous design experiment highlighted two points:

- Firstly, there are conditions in the surrounding context that are not formally observed through a typological analysis.

- And secondly, that allowing for these qualities in a design solution can improve the solution's response to site. Thus when designing an intervention on site, it is wrong to ignore these wider environmental conditions of context.

As a designer reflecting on this experiment, the typological approach is failed on both these accounts. For example, in repeated circumstances, the design had potential to become something more than only a response to the accessibility barriers identified within site. Also, sometimes a customised response was needed in the most neglected areas of site, as a responsive design intervention could have reinvigorated the area. So the experiment highlighted that in this situation the designer, as well as the research methodology, faces a problem.

- Firstly in terms of the designer - bearing in mind the main purpose for pedestrian infrastructural design is to improve pedestrian accessibility, to what extent should designs be responding to site in order to further create successful places?

- Secondly in terms of the research method - how can the methodology be adjusted so that the untapped potential of site is factored into the process of designing for pedestrian accessibility?

The following argument by Beattie and Downton about the role of the designer in creating successful places will be examined.

Beattie and Downton on place-making

Downton argues that the fundamental principle to successful placemaking is an awareness of how people use the surrounding environment. In this sense Downton characterises a 'good' place as being a place of significance to the people who use it. A 'good place' therefore is characterised by having meaning to others (111). According to Downton place and meaning are interrelated processes that occur over time. Thus as processes, their temporal quality means they simply cannot be designed or inserted immediately into the environment (116-7). 
"architects within the normal spectrum of their professional activities cannot make places. Rather, they can intentionally make the making of places more, or less, difficult (Downton 112)"

Downton argues therefore, that the role and authority of the designer in place-making is to,

"work in the realm of potential place"... and to... "design

settings that have the potential for becoming places (117)“.

This argument is significant as it challenges a fundamental principle behind creating typological solutions, or designing only for the immediate problem at hand in a pursuit for cost efficiency.

Beattie's argument further reiterates that it is important to recognise the untapped place-making potential hidden within site, by commenting on place-making in an urban context

"In some ways it appears that places, at least at this scale, are not created or made at all. Rather it may be seen that certain parts of the urban environment acquire a potential for becoming special through the existence of certain behaviour patterns... once in existence, its potential can be realised or enhanced by the designer (Beattie 25)."

Beattie continues, a designer is inevitably... "...presented with opportunities to observe the existing situation... and decide on an appropriate environmental change that will:

1. Reinforce or complete some existing positive trend in the surrounding area.

2. Extend a network or system that exists nearby.

3. Initiate the beginnings of a new network or system that may be continued in subsequent changes to the immediate environment.

4. In general make a positive contribution to the sense of place in the urban area as well as the specific building project (25)"

In conclusion, Downton's and Beattie's argument for creating successful places demonstrates there are responsibilities for a designer when making interventions within site. Their argument also reinforces the criticism identified in the earlier typological solution experiment; which is it is unlikely 'successful places' will be produced while the current design methodology used in this experiment for resolving accessibility barriers, relies so heavily on creating typological solutions. Subsequently, the new 'New Pedestrian Design Strategy' will be altered again after the final section of this chapter 


\subsection{Conclusions of the Typology So Far}

The conclusions made about the following two typological methods used in this thesis, which will now be discussed:

- Typological study of pedestrian accessibility in Central Wellington

- Standardised typological solutions

The Typological study of Pedestrian Accessibility

Typologies are useful in the form of a typological study as they allow the designer to assess the real world environment through a specific theoretical lens. In this thesis the lens was accessibility of the pedestrian realm. In terms of the pedestrian design strategy being developed in this thesis, the typological study is useful because it allows the designer to understand spatially in the built environment how certain spatial conditions affect pedestrian accessibility. In this sense, typologies function as an educational tool that can be used to inform a designer of how certain arrangements of spaces, or objects in space, might create barriers to walking.

Standardised Typological Solutions

When designing standardised typological solutions the research found there is a disconnection between 'theoretical typologies' and 'real world typologies'. To elaborate on this, 'theoretical typologies' are simplified as they operate in a realm without context or the complexities of the real world. Thus when a typology is created the diversity and uniqueness of the real world is lost.

For a designer approaching a complex problem on site for the first time, there is a seduction in the simplicity of the typology. Typologies seem easily transposable to the real world environment, partly because typologies are unique. They are a form of theory in a specialised medium, just like the real world built environment. This spatial similarity however, is what makes type so dangerous when designing. The fundamental principle that type is a 'method of simplification' makes type untransposable to the complex system of the real world; or at least not without having little response to the surrounding context. Pedestrian access typologies in the complexity of the pedestrian environment can only ever be a tool for understanding pedestrian accessibility, and also the spatial conditions in the environment that lead to accessibility barriers being created. The concept of a standardised typological solution will only ever be useful in resolving isolated problems on simple sites, if indeed these even exist. This is opposed to a customised intervention that responds to its surrounding context, explores possibilities for placemaking opportunities to occur on site, and is an intervention that improves the wider urban contextual problems. 


\subsubsection{Further Direction}

It was evident in 'Design Experiment 1 and 2' that the current design strategy needed to be adjusted for two reasons:

- Firstly, although a standardised typological approach still has some use due to it being much cheaper to implement, it cannot be used in a design that intends to respond to the qualities and conditions of site.

- Secondly, Beattie and Downton's argument highlights that the designer has a responsibility to ensure their interventions respond to the place-making potential that lies hidden within site.

Therefore two amendments were made to the 'New Pedestrian Design Strategy' that acknowledge the following findings:

- Firstly, a designer must embrace the existing untapped potential within a site, and go beyond resolving pedestrian accessibility barriers.

- Secondly, a designer must contribute to the place-making process, whereby the quality of any design intervention should respond to the site's surroundings in a way that encourages places of significance to form.

With these new changes in place the next research experiment is aimed at addressing the following question: 
4.

Conduct analysis of the greater urban context in order to see which accessibility barriers and place-making opportunities, which were identified through the cognitive surveys, are of most importance to improving the larger pedestrian and public space network.

What are long-term changes that need to be made within the larger urban context in order to improve: connectivity of the pedestrian network, as well as the quality of public space network, at a macro scale?

2.

Conduct a citywide survey using the cognitive interview method, to highlight the 'hot spots' where accessibility barriers are present in the city's pedestrian network. In particular between the residential catchments and the CBD.

‘Bottom up’ approach

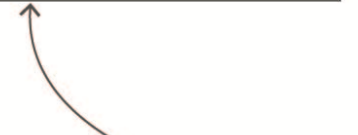

'Top down' approach
Within the key areas identified in the

previous macro urban analysis, particularly

where the cognitive survey data was

insufficient, conduct a further 'top down'

expert analysis at the micro scale. In order

to uncover any additional accessibility

barriers and areas of place-making

potential.

step

1.

Carry out a Typological study of pedestrian accessibility for Central Wellington as a

baseline for understanding in theory how

accessibility is affected by the design of the

built environment.
5. Locate the access typologies on site in order to understand the spatial principles that are causing each significant barrier. Therefore becoming familiar with the level of resources required to resolve the barrier. 


\subsection{Design Experiment 3: 'Place-making'}

When designing in the pedestrian environment there are areas off the footpath that can influence the place-making qualities of sites within the pedestrian network. However these qualities are often hidden within sites and are yet to be developed in the public space network. The aim of this design experiment therefore was to examine:

- $\quad$ Firstly, how these areas of place-making potential within site could also be developed.

- Secondly, how to do so whilst continuing with the initial process of resolving pedestrian accessibility barriers.

Therefore as a result, this experiment aims to improve both the quality of public space, and pedestrian accessibility in the built environment, together through one design process.

\subsubsection{Method}

The experiment used the same Salamanca Rd site 'accessibility barrier hot spot', as the previous experiment. This meant the same accessibility barrier problems applied as identified in Figure $25 \mathrm{pg}$ 85. These were the:

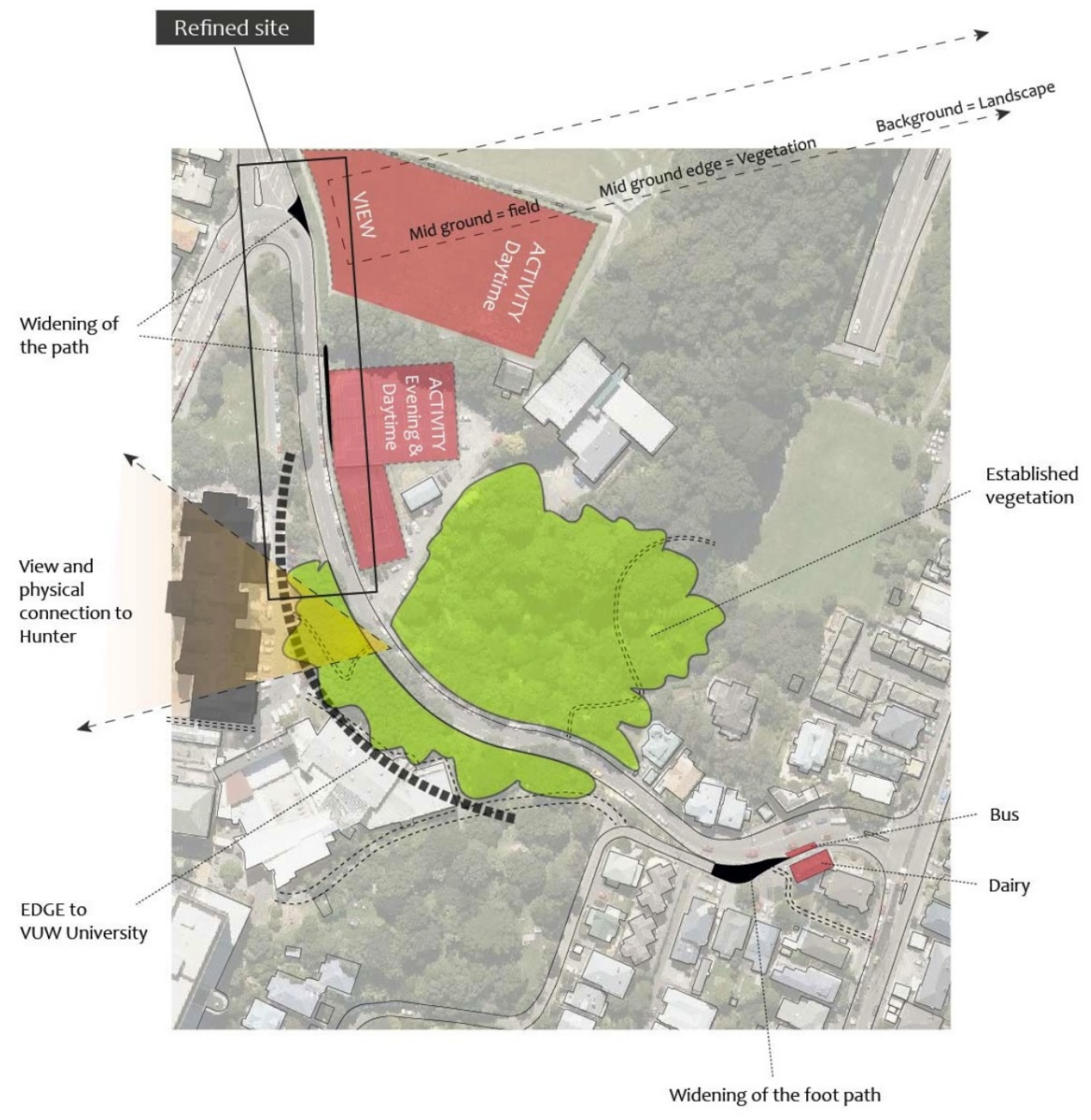

Figure 37: Areas of place-making potential within the Salamanca Rd site 
Hedge

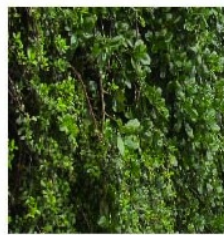

Established

trees
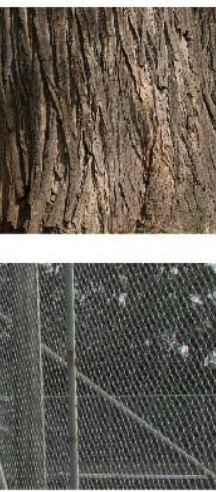

Tennis cour fencing
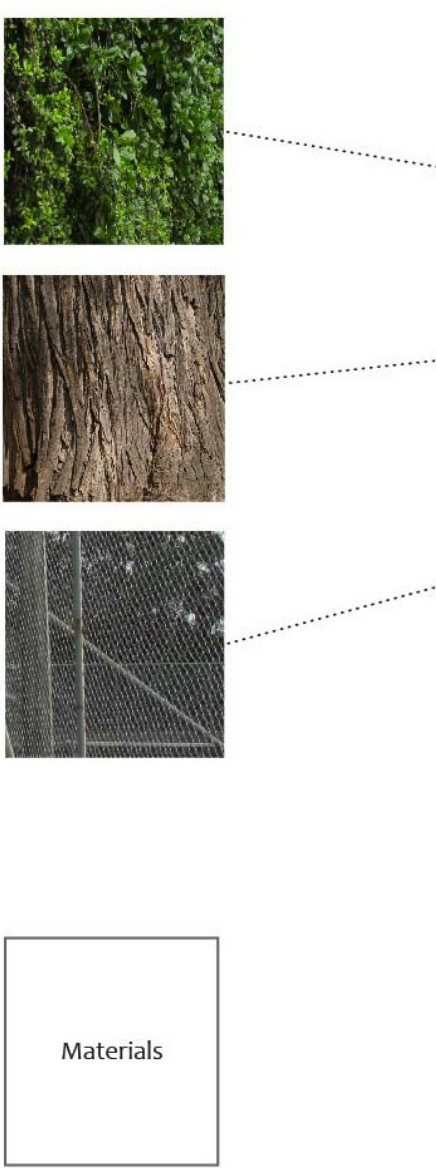

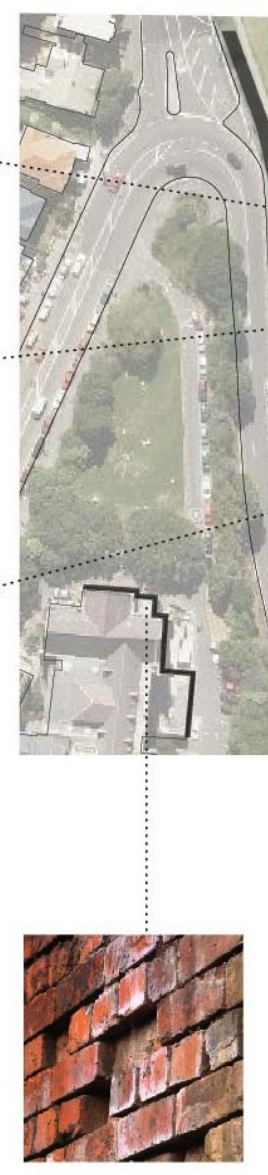

Iconic University Hunter building
1. Lack of shelter from weather

2. Lack of social safety in the evening

3. Lack of lighting in the evening

There are a couple of things to note in this experiment.

- Firstly, the second accessibility barrier will not be addressed as it requires investigation into wider macro scale factors that occur outside the site's boundary (later on in' Design Experiment 4' this macro scale will be addressed).

- Secondly, for the purpose of this experiment, the site was reduced further to the area outlined in (Figure 37), and the design will focus on resolving specifically the' lack of shelter from weather', accessibility barrier 1 .

\section{Step 1}

To begin with, the attributes of site that had place-making potential were identified in the surrounding areas off the footpath. This was done using a 'top down' expert approach to site analysis (Figure 37). Note the same node consisting of a bus stop, dairy, and public space, which was identified in the previous experiment (Figure 35 pg101), would have been identified in this analysis if it was included in the new site boundary. 


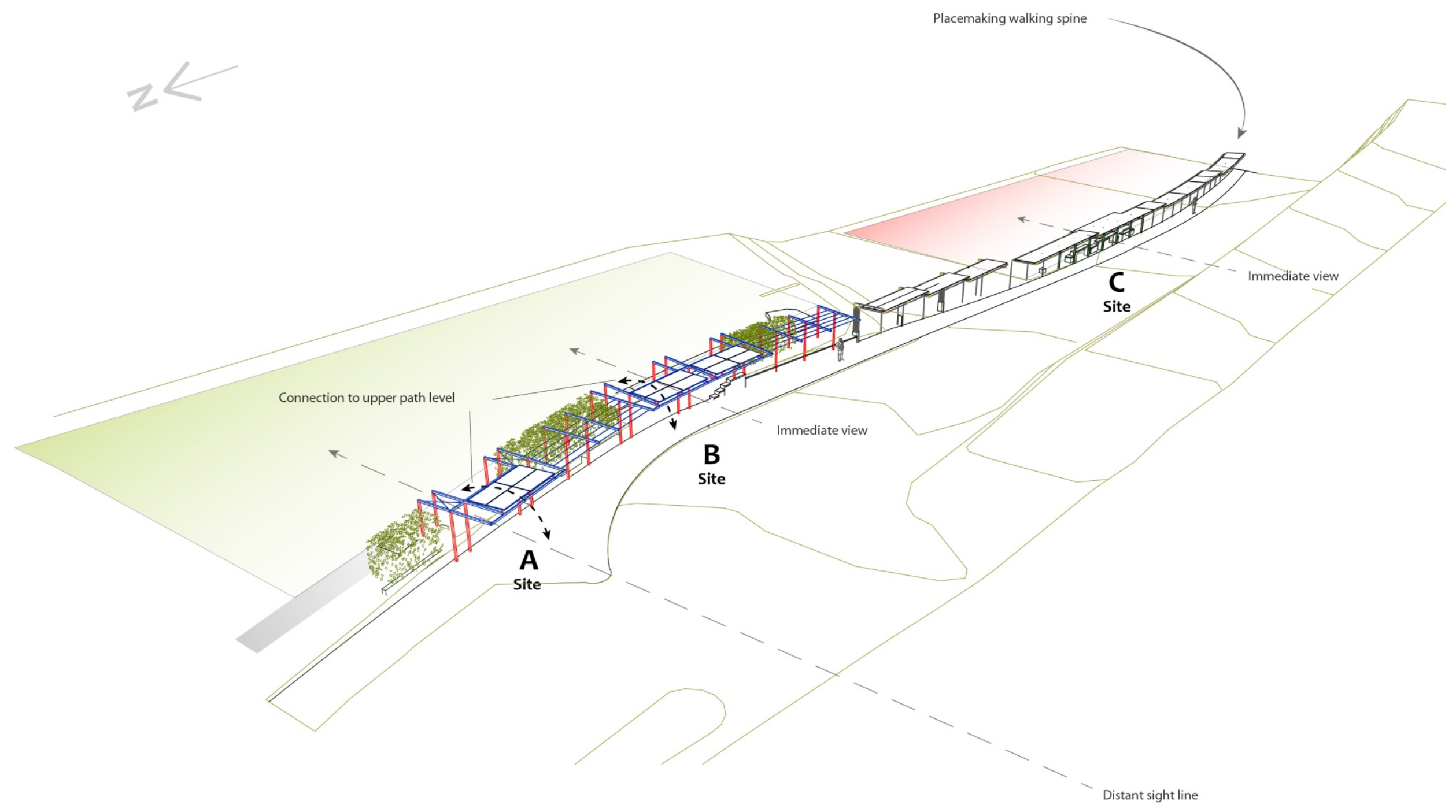

Figure 39: Design response - 'Lack of shelter from weather'

111 
Following this, the contextual material qualities within site were noted (Figure 38). This was necessary to ensure any interventions designed later on were responsive aesthetically and formally to the surrounding context, which was a criticism of the previous typological design methodology. In response the design in (Figure 39) was created.

\subsubsection{The design}

In addition to the accessibility barriers present in the site, the 'top down expert' site analysis found that the existing hedge at sites A and B (see Figure 39 running North - South along the Eastern side of the rd) was causing three problems:

- Firstly, blocking sunlight onto the footpath during the morning through to lunchtime.

- Secondly, blocking views Eastward out to city, as well as views to the mid ground open green space area.

- Thirdly, it prevented access to an adjacent upper level path running next to a bowling green.

The following images demonstrate how through resolving the accessibility barrier of 'no shelter from weather', the additional place-making qualities of site could be incorporated into the design effectively:

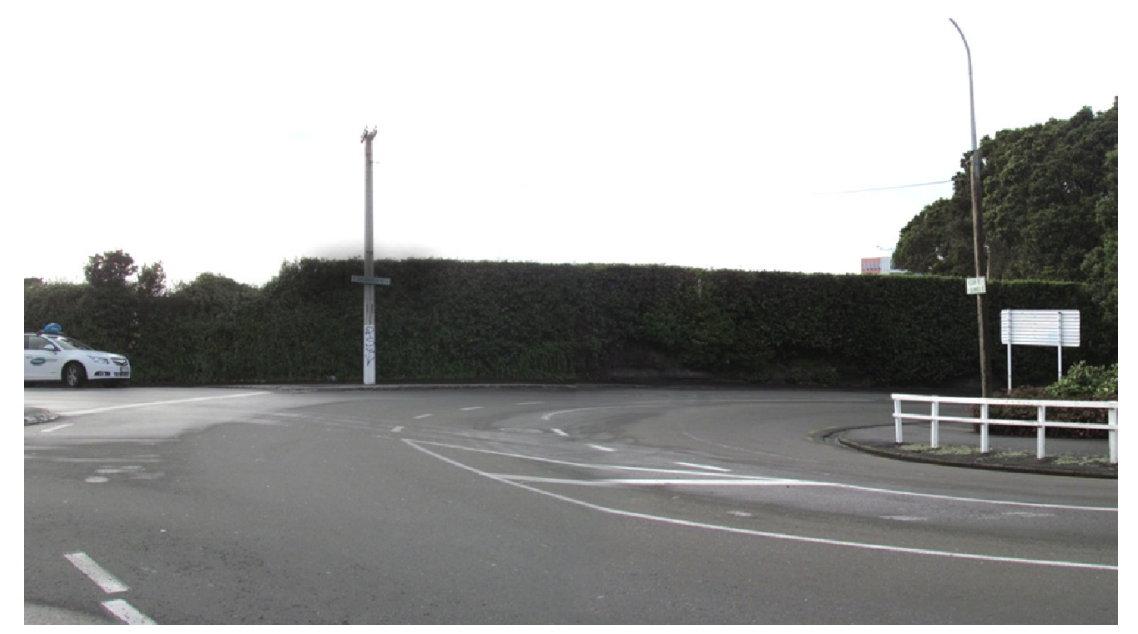

Site $A$

Figure 40: Before -> After 


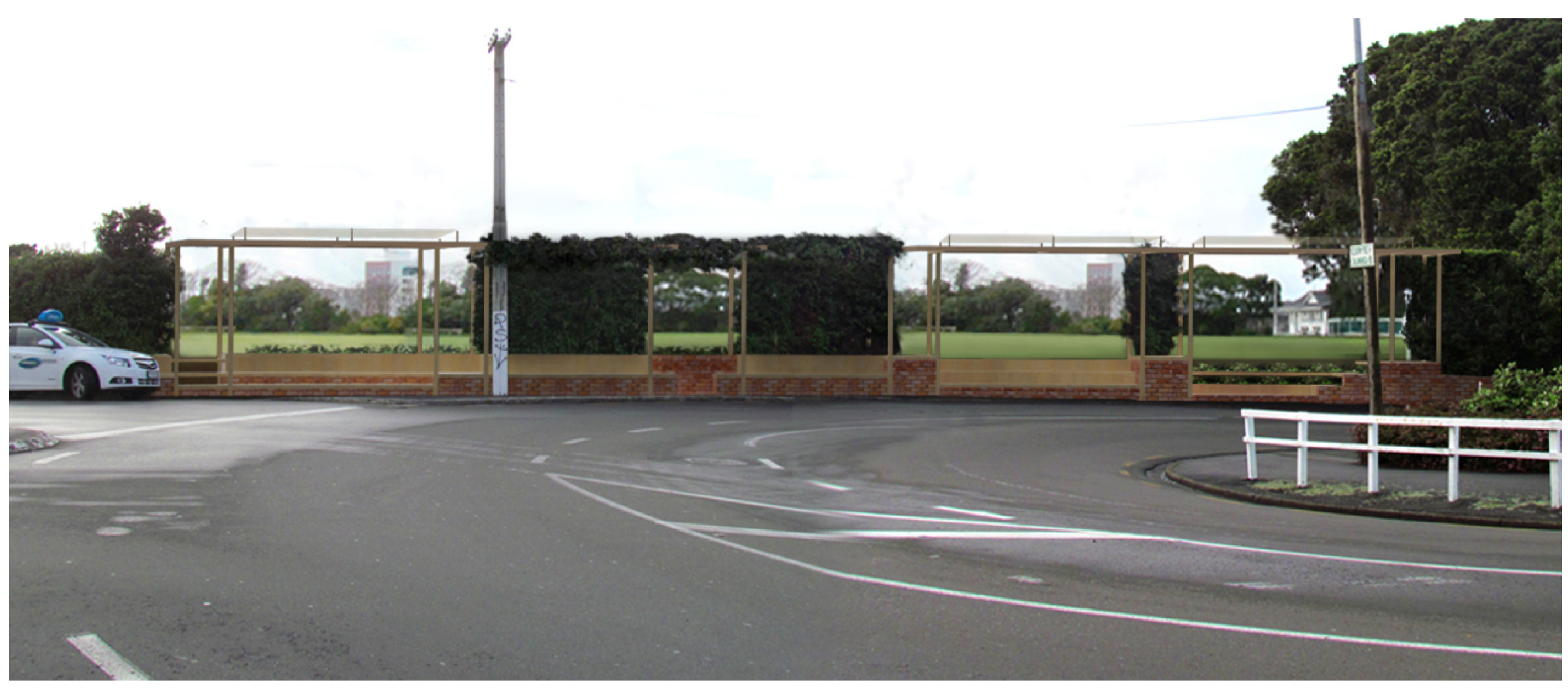

Removing areas of the hedge frees up views to both the adjacent green space and city skyline. 


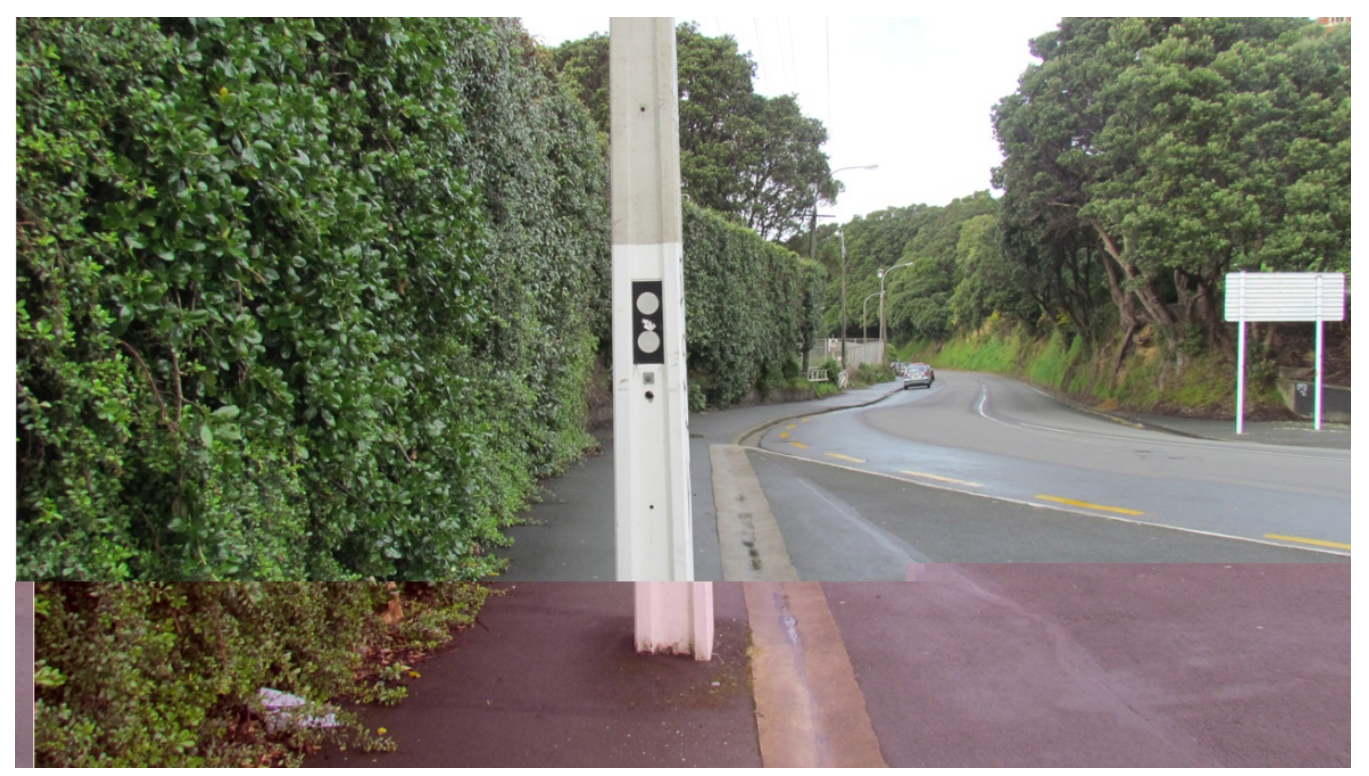

Figure 41: Before -> After

Site A

The design's vegetation canopy shows responsiveness to the character of the surroundings through embracing the formal quality and materiality of the large hedge. 


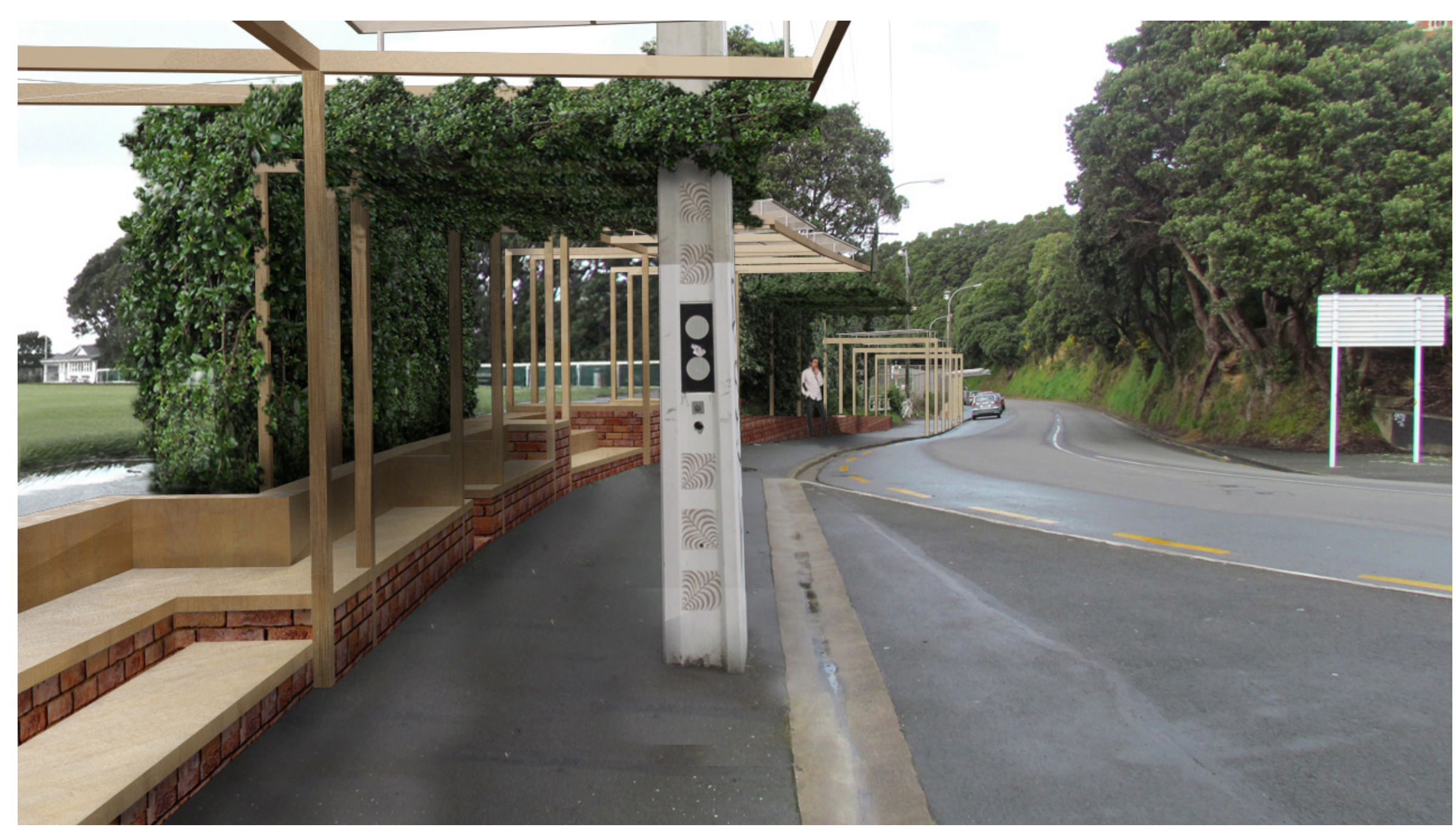




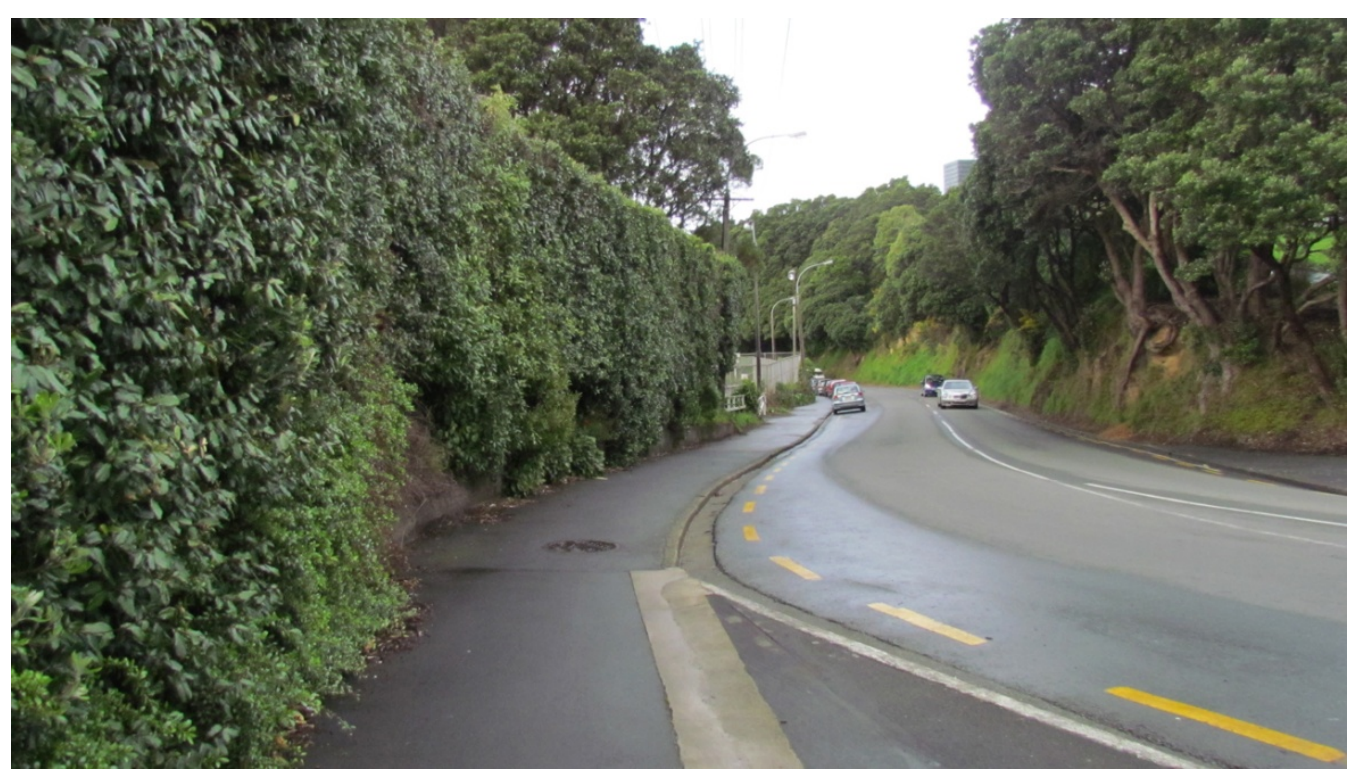

Figure 42: Before -> After

Site $B$

Integrating terraced seating under the canopy structure reconnects the upper level path with the road-side footpath. 


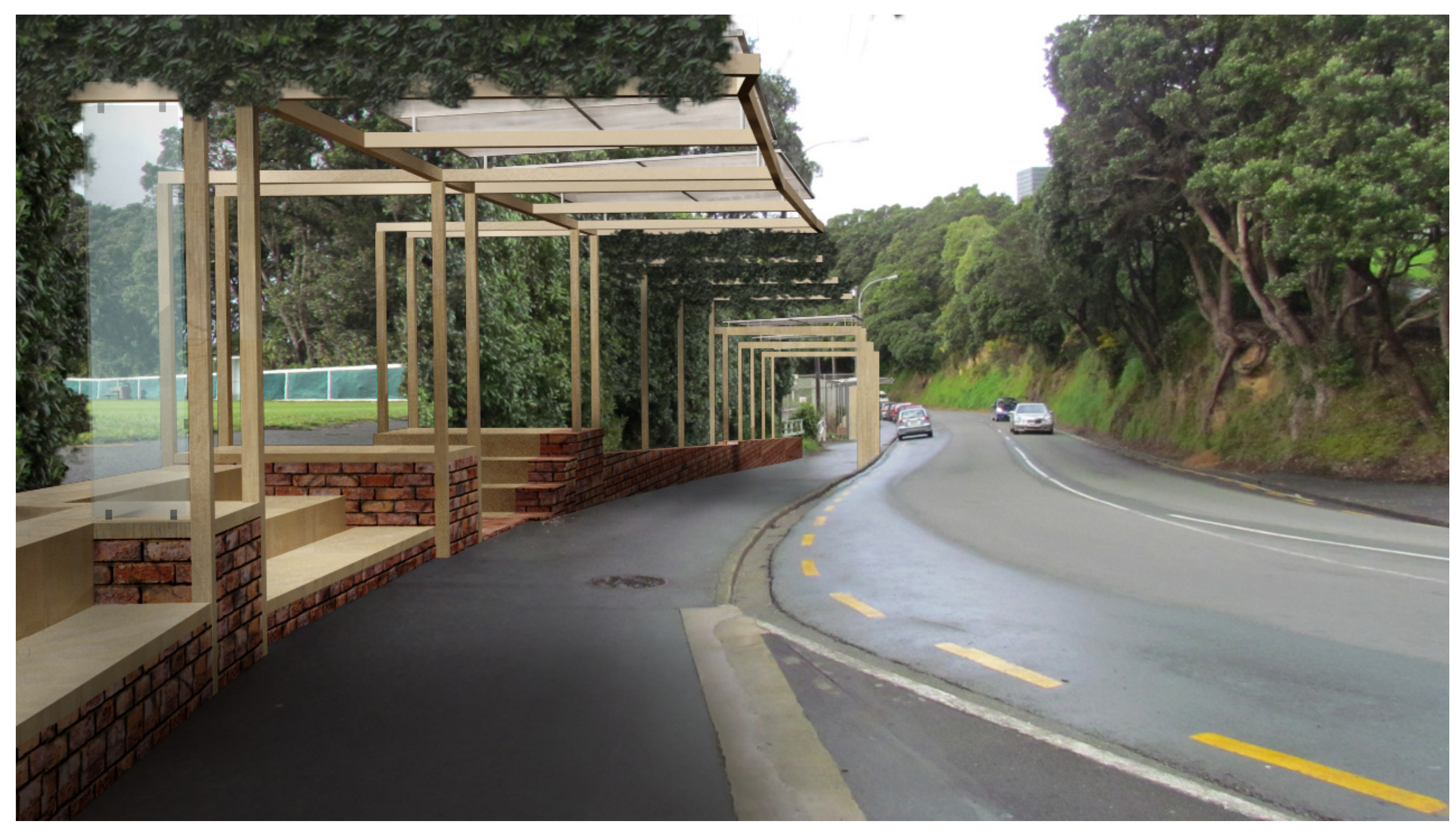




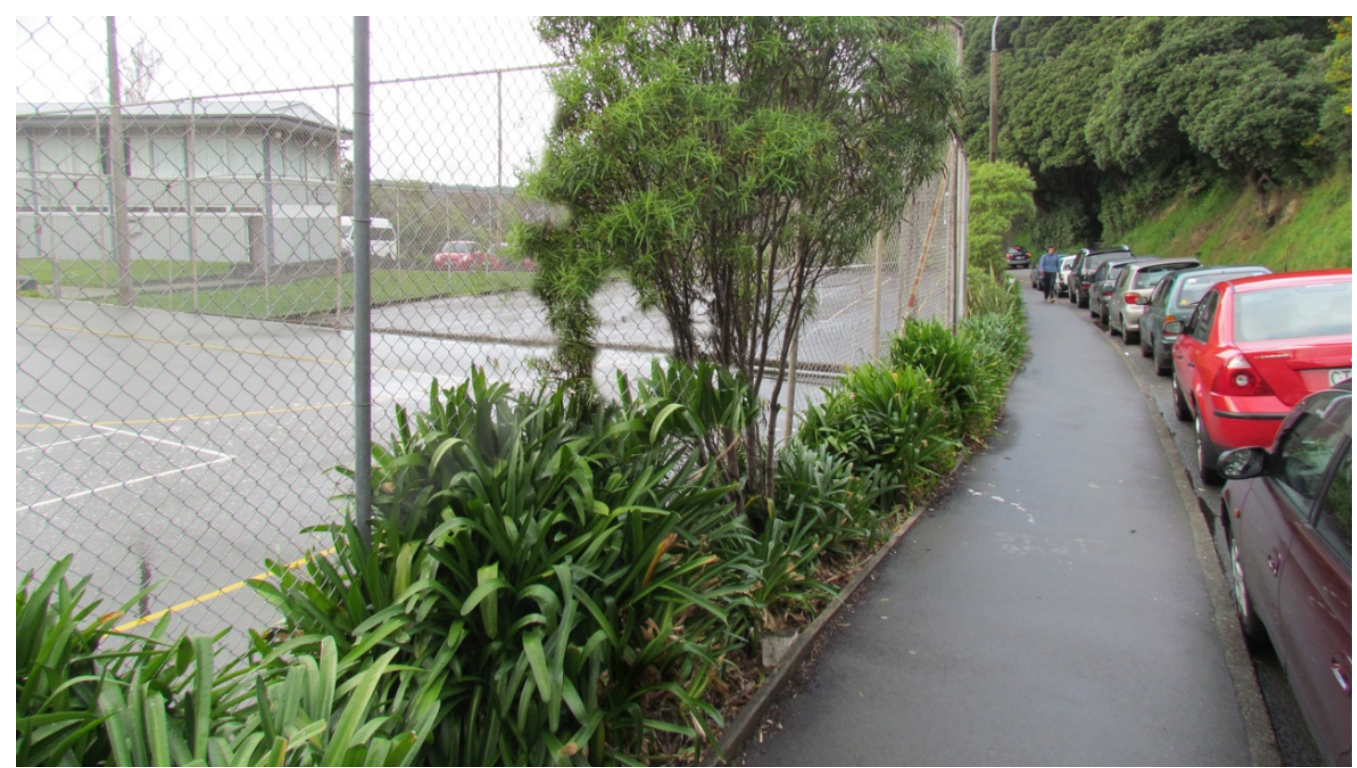

Figure 43: Before -> After

Site C

By having an awareness of the place-making qualities of site, such as the frequently used sports courts adjacent to the footpath, a row of informal seating was easily integrated into the shelter solution. 


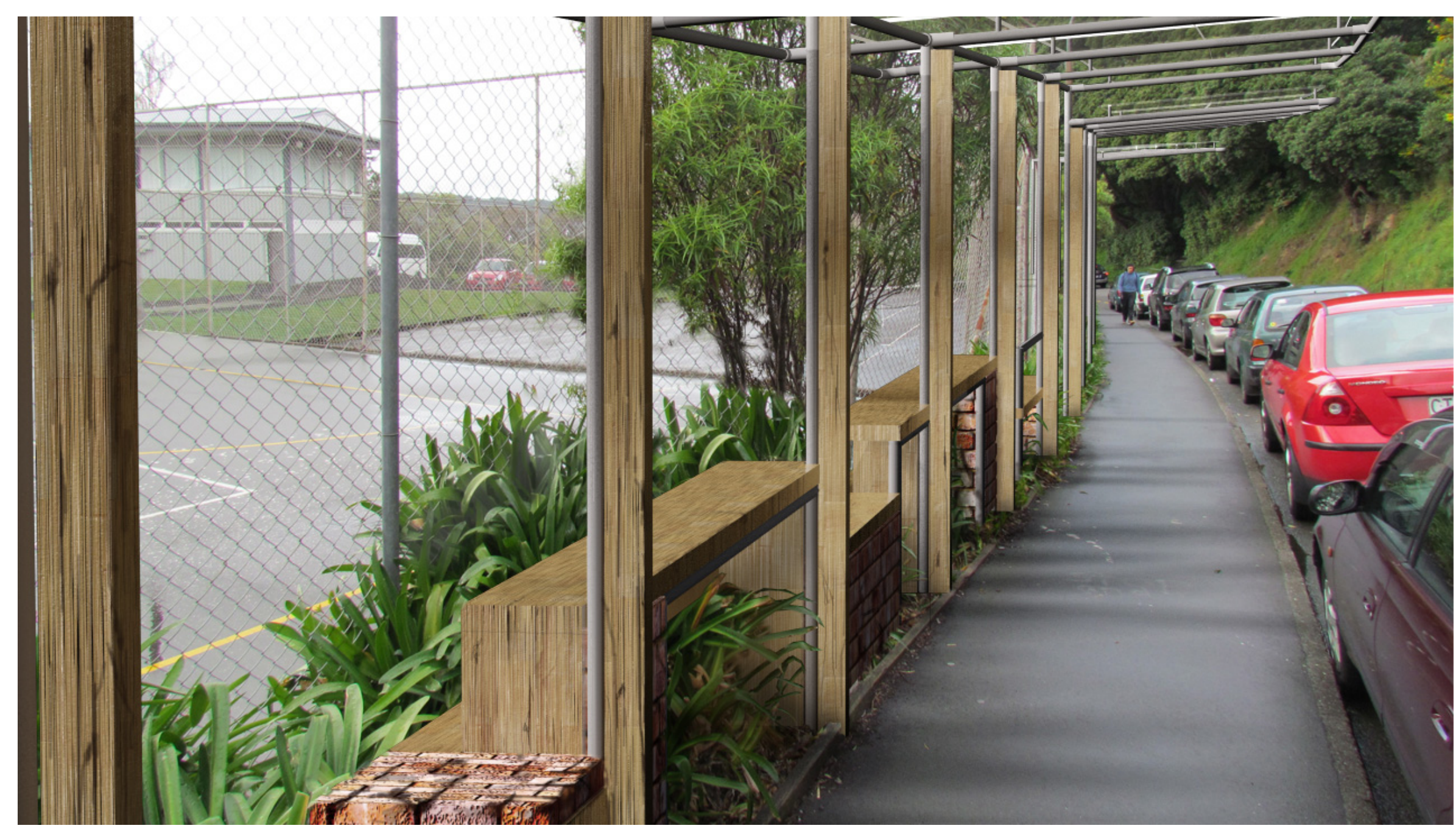




\subsubsection{Findings}

The experiment highlighted that having awareness of the place-making qualities of site whilst resolving pedestrian accessibility barriers, clearly results in a much more effective design intervention that is responsive to its surrounding context, and only for a slight increase in cost. Furthermore, this increase in cost is negligible relative to the additional cost savings gained through the accuracy of the cognitive survey. Note that the cognitive survey technique can identify the key barriers affecting accessibility within a site, and thus prevent superfluous use of resources i.e. in this experiment re-surfacing the footpath or path widening which were not identified as accessibility barriers.

These findings are significant to the pedestrian design process in Central Wellington as it demonstrates that interventions to the pedestrian network can be more than just a solution to an accessibility barrier. In fact, by resolving accessibility barriers to walking, authorities are given a new opportunity to: identify areas in the pedestrian network that have placemaking potential, develop these areas and improve the quality of the public space along a pedestrian route, and over time significantly improve the quality of the pedestrian environment. This widens the potential of small macro scale pedestrian infrastructural improvements to go beyond only addressing barriers to walking, which is currently not realised in
Central and Wellington local authorities' process for designing pedestrian infrastructure.

\subsubsection{Further direction}

So far two design approaches have been used in this thesis:

- Design Experiment 2: the standardised typological approach

- Design Experiment 3: the customised place-making approach,

Both however, have been limited to only improving accessibility and placemaking at the micro scale within the 'barrier hot spot'. This is problematic because:

- Firstly, macro scale accessibility barriers such as journey proximity and connectivity cannot be resolved through a micro scale intervention alone as they require the wider macro context of site to be understood. For example, micro scale interventions can resolve some accessibility barriers on site, improve the relationship between the 'footpath' and the surrounding context, and as a result, encourage places throughout the pedestrian network to evolve over time into places of significance to pedestrian commuters. However, there is a limit as to how far accessibility and place-making in the wider context can be improved through this scale of intervention. 
- Secondly, designing for the micro and macro scale together results in a more cost efficient process than focusing on the micro scale alone. This is because until a comprehensive understanding of the pedestrian accessibility in the wider context is known, a designer cannot be certain which barrier 'hot spot' is the most cost effective place to spend resources. As there may be other routes nearby that could be improved as an alternative but at a lower cost.

In conclusion this experiment highlighted that before any design interventions are inserted on a site, it is important to understand the larger context that the barrier hot spots sit within, and in particular, how accessibility is affected on the macro scale. As a result, the 'New Pedestrian Design Strategy’ was altered accordingly. 
$4 \cdot$

Conduct analysis of the greater urban context in order to see which accessibility

barriers and place-making opportunities,

which were identified through the cognitive surveys, are of most importance to improving the larger pedestrian and public space net-

work.

What are long-term changes that need to be made within the larger urban context in order to improve: connectivity of the pedestrian network, as well as the quality of public space network, at a macro scale?

Conduct a second more detailed cognitive survey specific to the wider 'hot spot' area in order of highlight the barriers and

perceived place-making qualities of all the pedestrian routes throughout the site. This is important because other routes may present a more affordable alternative to

resolving the 'barrier hot spot', or may present better opportunities for place-making.

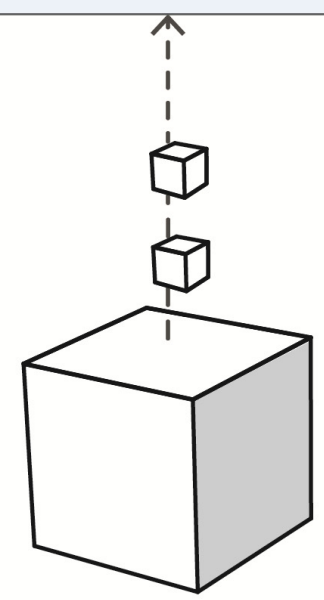

Library of pedestrian: - Access typologies - Land use typologies work. In particular between the residentia catchments and the CBD.

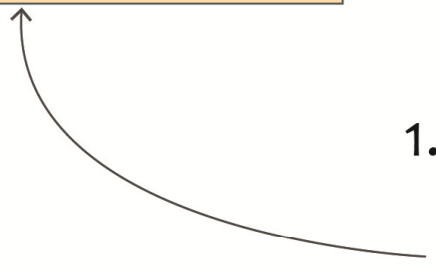

1.

Carry out a Typological study of pedestrian accessibility for Central Wellington as a baseline for understanding in theory how accessibility is affected by the design of the built environment.
5

Within the key areas identified in the previous macro urban analysis, particularly where the cognitive survey data was insufficient, conduct a further 'top down' expert analysis at the micro scale. In order to uncover any additional accessibility barriers and areas of place-making potential.

6. Locate the access typologies on site in order to understand the spatial principles that are causing each significant barrier. Therefore becoming familiar with the leve of resources required to resolve the barrier.
'Bottom up' approach

‘Top down' approach 


\subsection{Design Experiment 4: 'Rethinking the Larger}

\section{Pedestrian Network}

The purpose of the final design experiment was to create a long-term strategy for improving pedestrian accessibility at both the micro scale and macro scale. The experiment was broken into two parts:

- Firstly, to collate what was learnt in the previous design experiments into one multiple staged strategy for researching, designing, and implementing pedestrian infrastructure.

- Secondly, by analysing the larger urban context that the Salamanca RD 'barrier hot spot' sat within (Figure 44), the experiment tested how accessibility and place-making within the pedestrian network could be improved at both a micro scale, within the barrier hot spot, as well as at a macro scale in the wider urban context. This is significant as all of the previous experiments failed to consider the pedestrian network at this larger macro scale.

\subsubsection{Method}

To begin with, more research was needed of the wider context surrounding the Salamanca Rd barrier 'hot spot', in order to understand

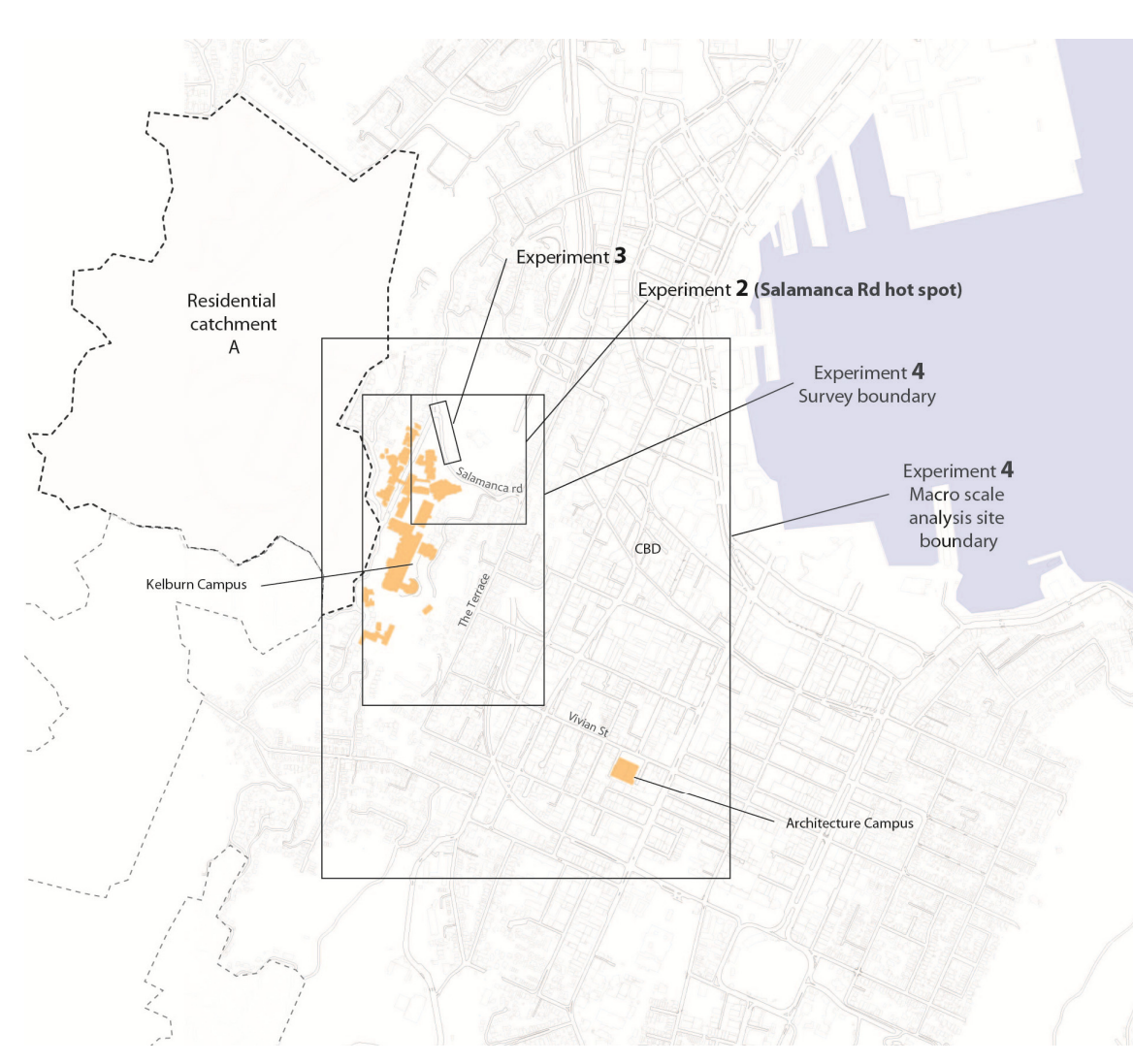

Figure 44: Site boundaries for 'Design Experiments' 2, 3, \& 4

the nature of the accessibility barriers that were present, as well as the places that have place-making potential throughout the wider site. The research was carried out using a combination of 'bottom up' and 'top down' approaches, which will now be discussed. 


\section{'Bottom up' Qualitative Analysis: Survey Results}

Unlike the previous cognitive survey in Design Experiment 2, which lacked depth as it covered the Central Wellington area broadly, the new cognitive survey was conducted over a much smaller site. The new site boundary was chosen specifically focusing around the main pedestrian routes that connect residential catchment ' $\mathrm{A}$ ' to the city (Figure 51 pg129). The survey demographic interviewed was $1^{\text {st }}$ year architecture students at Victoria University of Wellington. The demographic was chosen for two reasons:

- $\quad$ This user group were easy to access.

- The students regularly have classes at both Architecture and Kelburn University campuses and therefore often commute through the site area (Figure 51 p129).

In total 42 surveys were conducted and the results were overlaid together using a computer. See Appendix F pg230 for an example of the survey.

\section{Deciphering the Accessibility Barriers}

In the first stage of mapping, the results revealed a range of accessibility 'barrier hot spots' and areas considered as having place-making potential. These are the darker lines in Figure 46 and 47 respectively (note the difference between the two figures is very subtle). The data, however, was less useful until it was sorted into accessibility categories, thus allowing the most significant barriers to be highlighted (these are the barriers that were raised the most number of times in the survey). The sorting process was as follows:

- The barrier map was broken into hot spots specific to each accessibility sub category;

- $\quad$ Any barrier in the same area that was raised less than 4 times (this was \%10 of the survey sample size) ${ }^{4}$ was disregarded;

- $\quad$ Any barrier in the same area that was raised 4-8 times was labelled as a 'moderate barrier'.

- $\quad$ And any barrier in the same area raised 9+ times was labelled as a 'significant barrier'.

Figure 48 shows the most problematic area was across zone A and B, which contains a combination of significant and moderate barriers over a length of 300-400m

\footnotetext{
${ }^{4}$ Note, in practice, when the survey has a larger sample size the thresholds of the categories would be adjusted in order to control for the statistical margin of error for
} the chosen percentile confidence. 


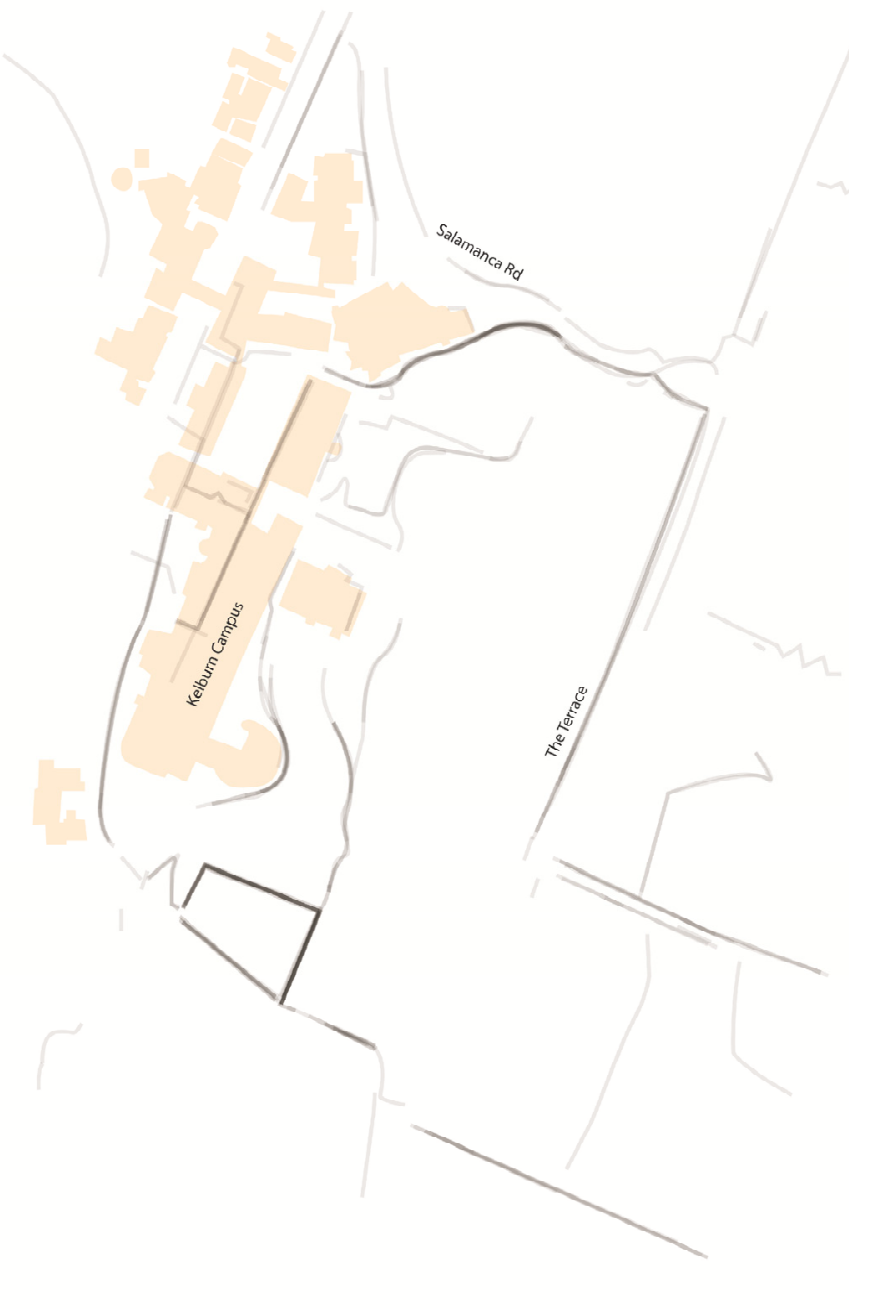

Figure 46: Overlay map of place making

potential as perceived by the respondents

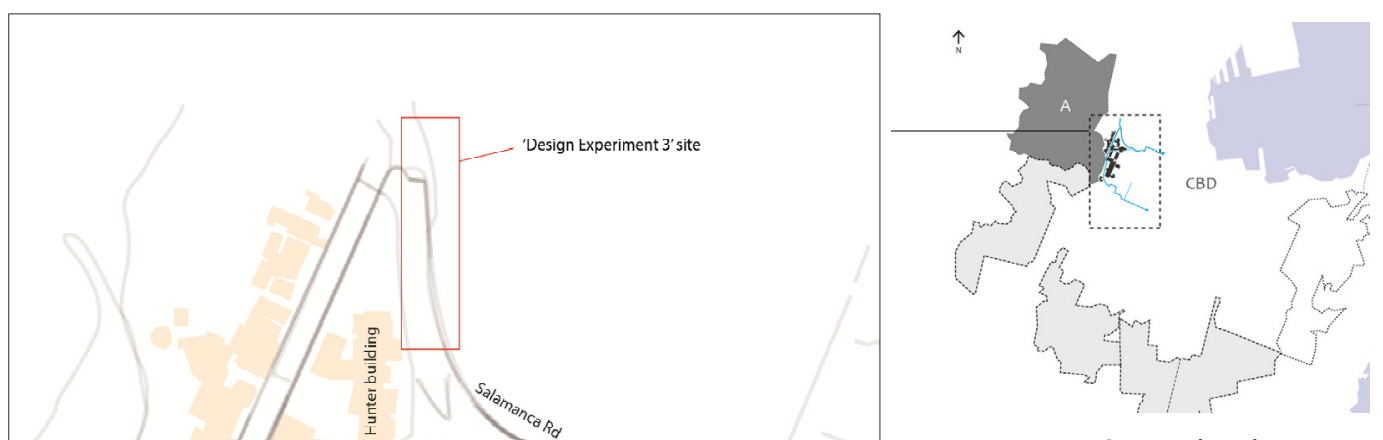

Location map: Survey in the wider context

Figure 47: Overlay map of all accessibility

barriers as perceived by the respondents 


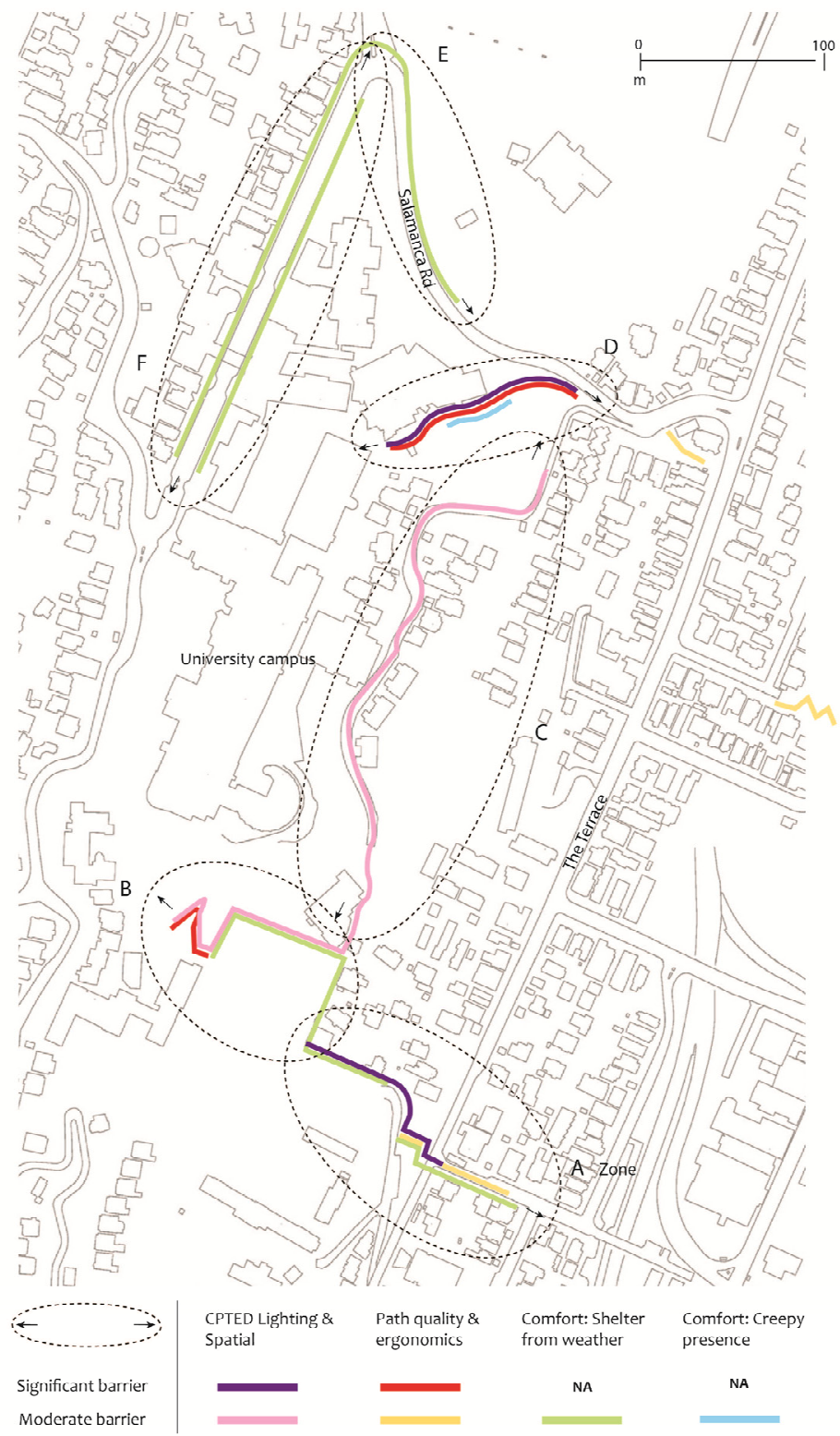

\section{Place-making Potential}

In the next step the place-making potential of site was established. The same method of sorting the raw survey data, which was used to highlight the significant barrier, was used again to isolate the significant areas of place-making potential (Figure 50). Listed below are the relevant spatial conditions within site that I considered to, embody place-making potential, and were unique to site in that they could not easily be recreated through inserting a new built intervention:

- Significant sun and shade

- External shelter from weather (provided by significant natural or man-made forms i.e. vegetation, typography, or existing buildings)

- Social interest from the presence of people or activity on nearby sites

- View shafts to areas of social, natural, or built interest

- Desirable vegetation

Figure 50 shows one particular area, Waiteata rd, was identified by the respondents as having moderate place-making potential due to 'significant views out over the city. In addition to this, the 'top down' analysis also noticed this area as having the strong place-making qualities (Figure 49) as it was:

- A relatively undeveloped site by the University,

Figure 48: Map of accessibility

barrier hot spots
- Located along a neglected pedestrian terrace that provided North South connections across the base of University campus. 


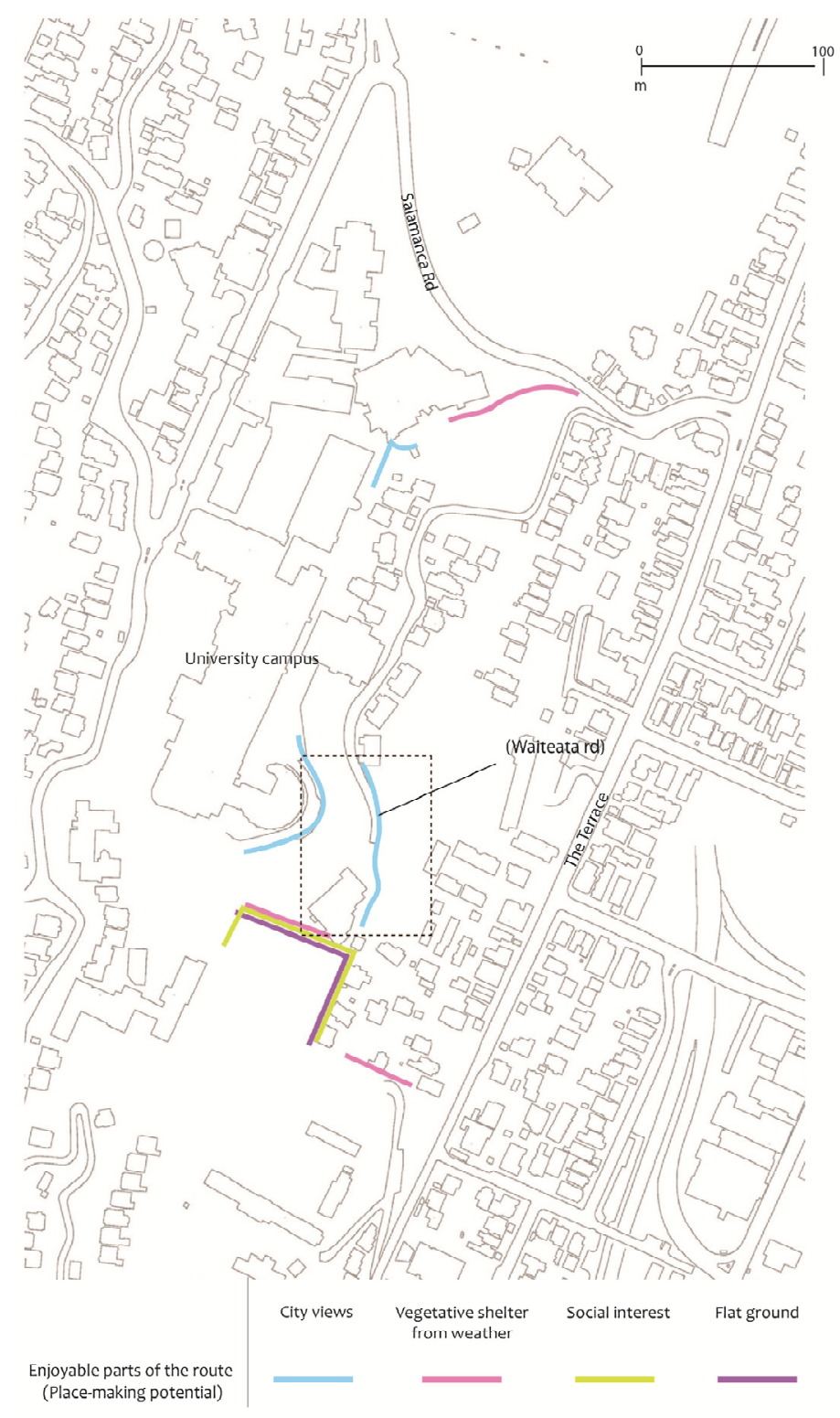

Place-making area 3
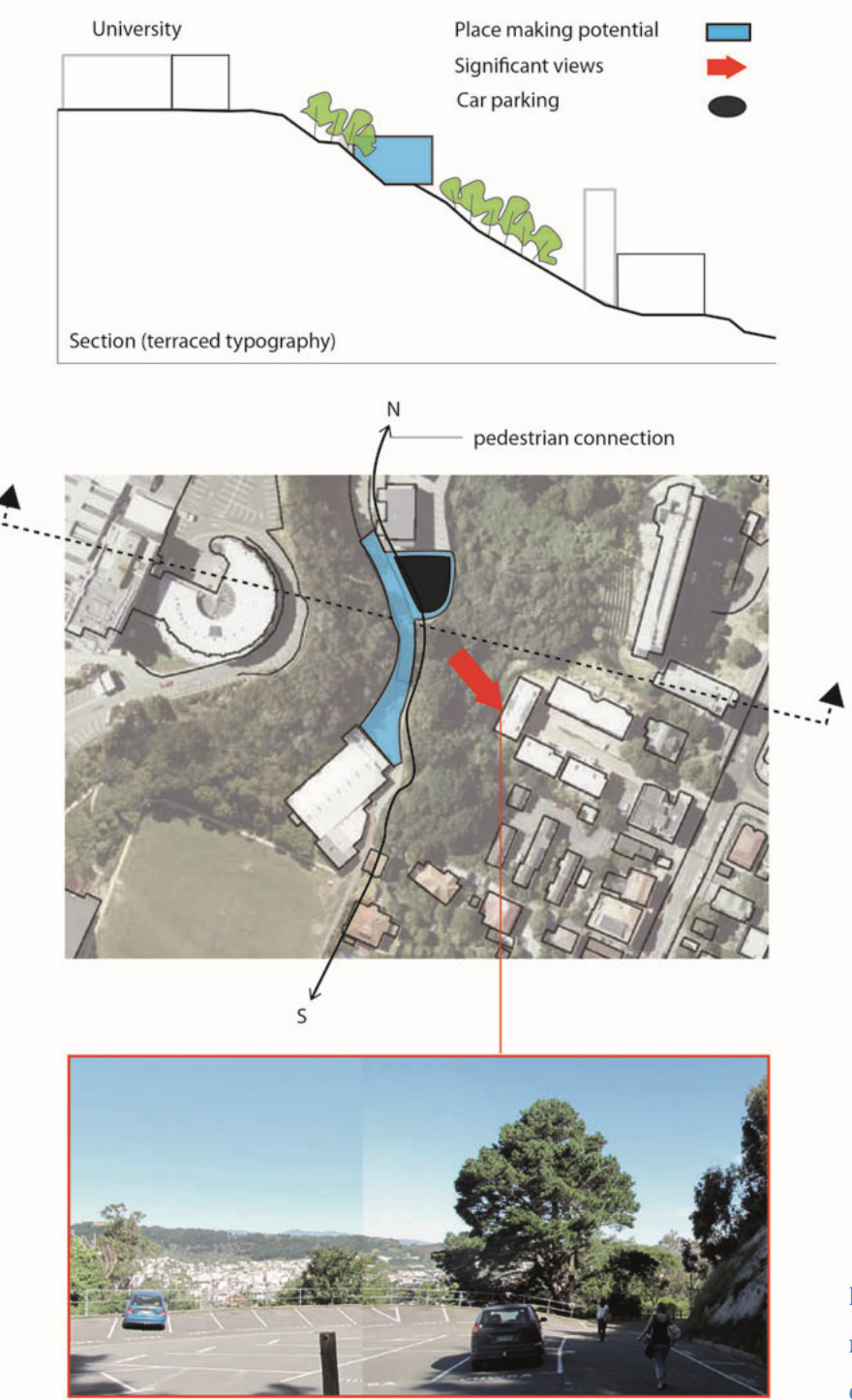

Figure 49: Placemaking qualities on Waiteata rd
Figure 50: Map of

areas of place

making potential 


\section{'Top down' expert analysis}

Having used the 'bottom up' qualitative approach to identify a range of accessibility barriers and areas of place-making potential on site, the next step was to conduct a larger 'top down expert' urban analysis of the pedestrian network. This was for two reasons:

1. To understand how the significant barriers and place-making areas identified in the surveys at a micro scale, relate to accessibility and place-making within the wider pedestrian network at the macro scale.

2. To establish a hierarchy of 'implementation urgency' for the barriers and place-making areas within the site. This was to educate authorities on the order these areas should be addressed if they are to achieve maximum cost efficiency and effectiveness when implementing changes to the pedestrian network.

\section{(Macro scale) The Pedestrian Network in the greater urban context}

\section{Step 1}

To begin with, the major pedestrian routes through site were identified using 2008 pedestrian count data supplied by Wellington City Council (Appendix G pg233) these were:
- The major pedestrian routes connecting Residential Catchments B, C, D, E, to Kelburn University (Figure 51).

- The main pedestrian route connecting Residential Catchment 'A' with the CBD (Figure 51).

Note the most direct route from Residential Catchments B, C, D, E, to the University is through the Southern end of site boundary. Thus this area of site has an important role in affecting accessibility on a macro scale for commuters walking from the Southern end of Wellington Central up to the University.

Step 2

Following this, the most underutilised and frequently used routes through site where highlighted using Figure 52 (which was also derived using data from the Wellington City Council pedestrian counts Appendix G pg233. The site boundary for the macro scale analysis is shown in Figure 51. The observations from this analysis were:

- Firstly, of the two connections that were providing key access from Residential Catchment ' $A$ ', past the University Campus, and then on to the CBD; the Vivian St connection experiences significantly lower pedestrian volumes relative to the Salamanca Rd connection.

- Secondly, therefore, Salamanca Rd is the primary pedestrian connection linking Residential Catchment 'A' to the CBD. 


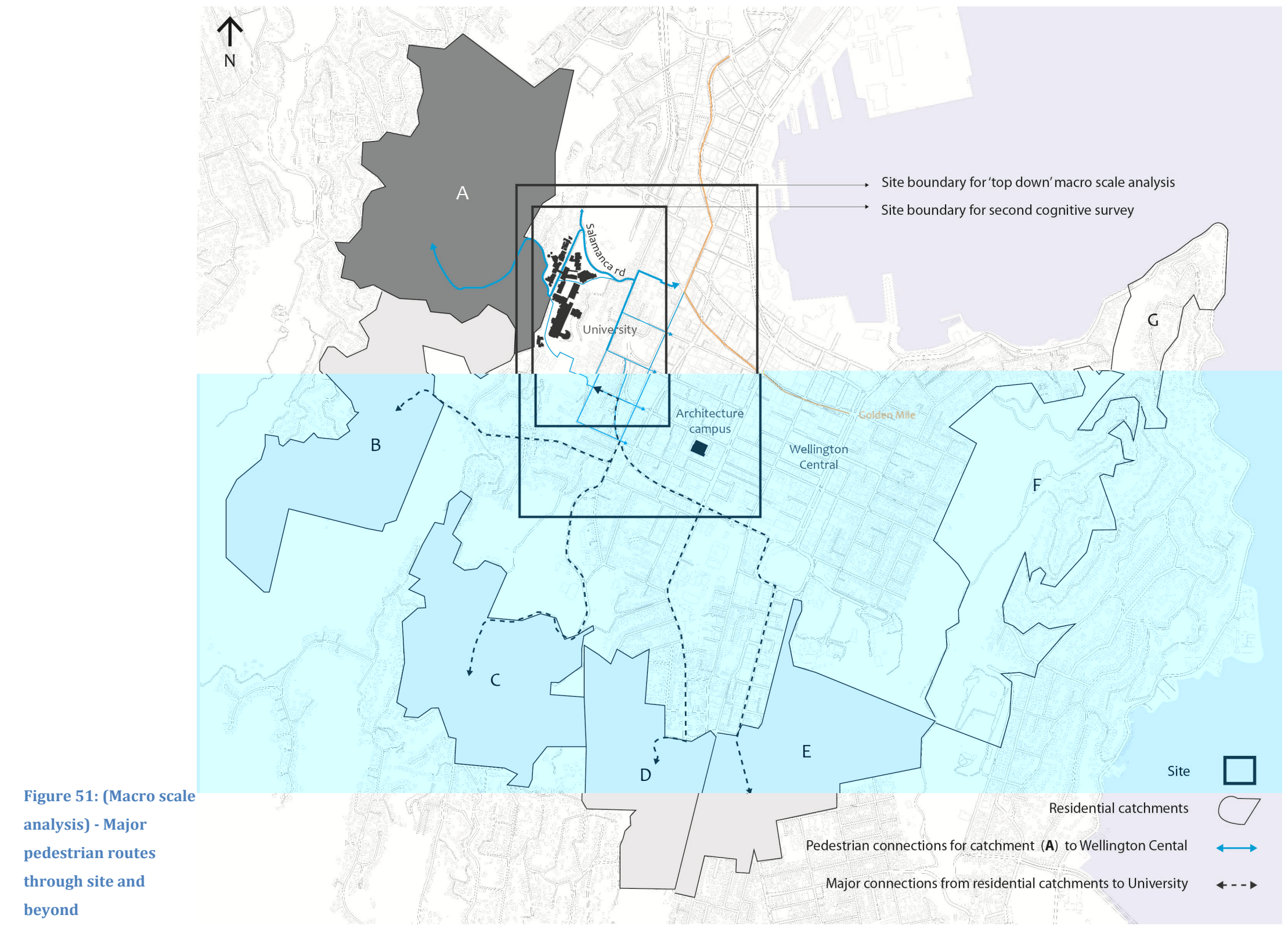




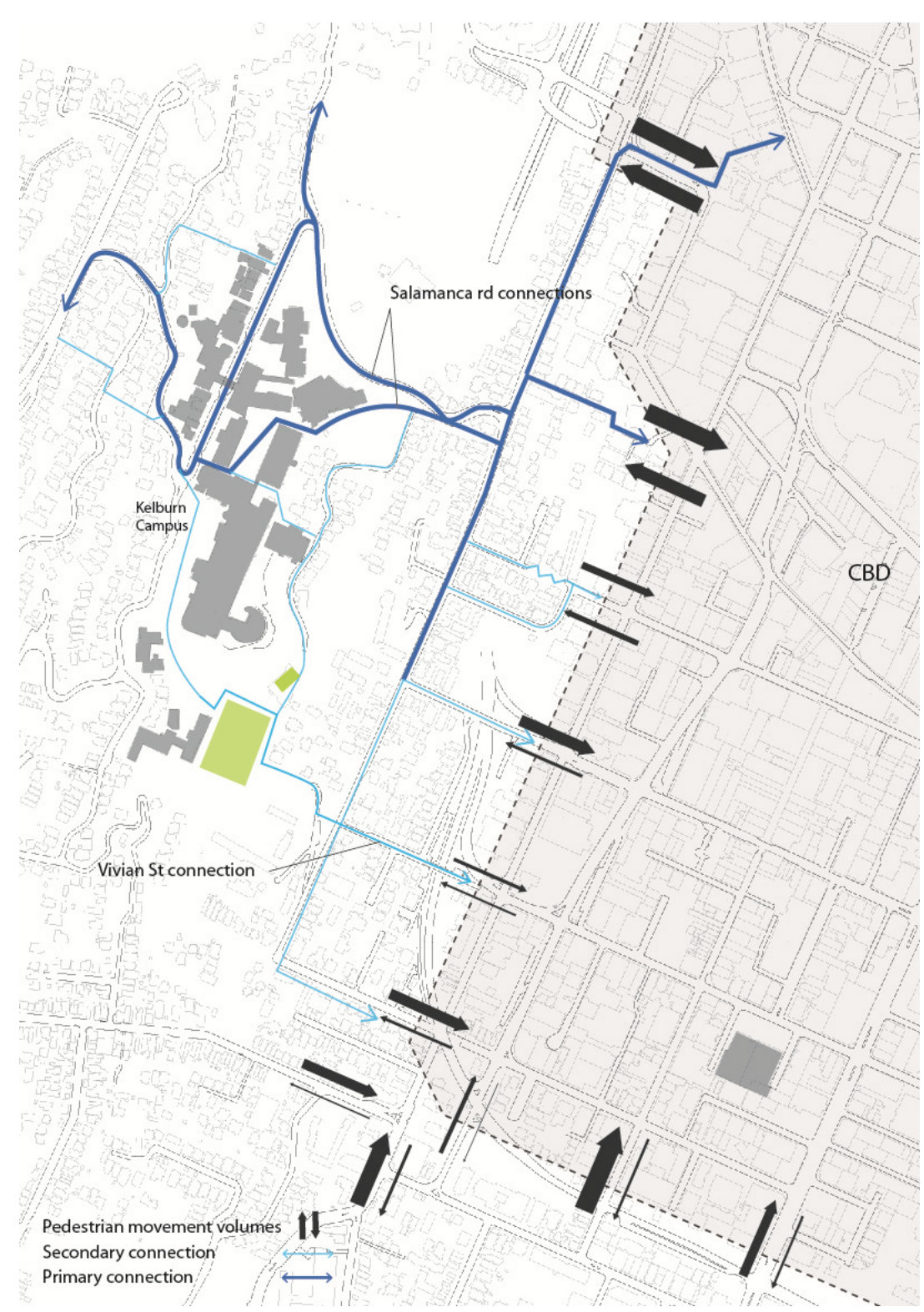

Figure 53: (Macro scale analysis) - Hierarchy of pedestrian volumes
There is an issue, however, with using the 2008 pedestrian counts, which are currently out of date, as there have been two significant changes affecting pedestrian volumes through site which need to be considered.

- Firstly, a high-density housing complex accommodating 374 University students (VUW), Te Puni Hostel, has been developed at the Western end of the Vivian St Connection (Figure 53).

- Secondly, first year Design and Architecture students now commute from Architecture Campus to Kelburn Campus for lectures multiple times per week (Figure 53).

These factors have raised the importance of the Vivian St connection as a significant route within the wider pedestrian network.

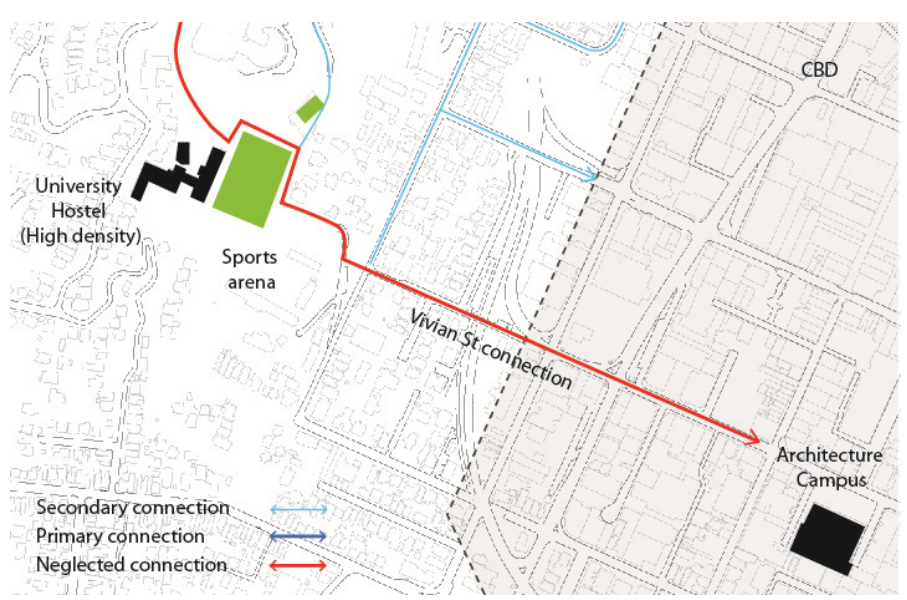

Figure 52: Neglected connection to CBD - Vivian St 
(Macro scale) The Public Space Network in the Greater Urban Context

Step 3

The next step of the macro scale analysis was to examine the distribution of public space through both the site and the adjacent CBD area. This was in order to see whether there were any notable relationships between the primary Vivian St and Salamanca Rd connections heading up to the University, and the public space network (Figure 54). The map highlights there is an absence of public space surrounding the Vivian St connection, which further reinforces that the Vivian St Connection is a neglected pedestrian route.

\section{Step 4}

The public space analysis was then compared with the previous analysis of pedestrian volumes. The analysis identified two key nodes where viable improvements to the public space network could be made; particularly as the nodes occurred at the meeting of two significant pedestrian routes (Figure 55). The nodes were:

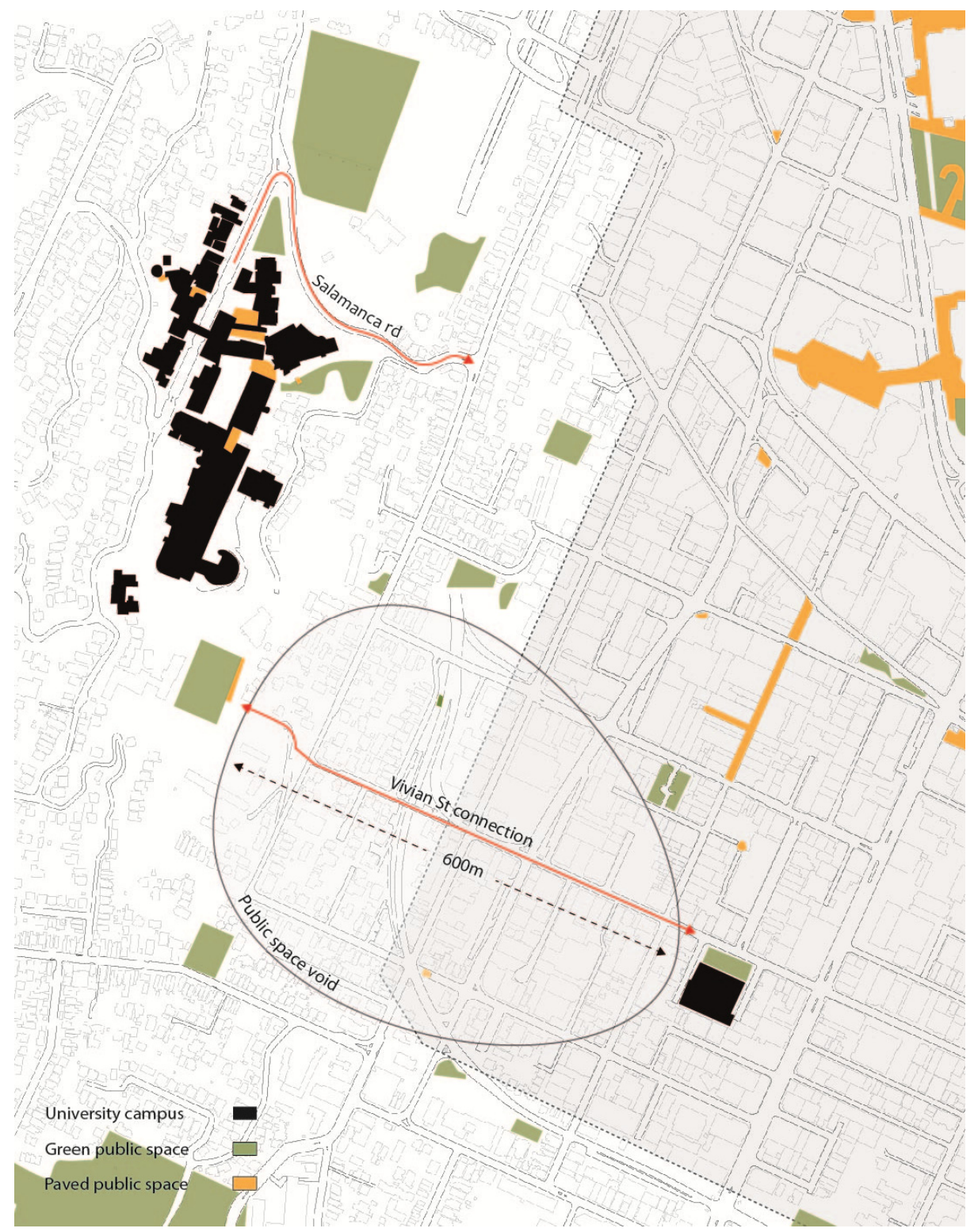

Figure 54: (Macro scale analysis) - Public space and green space 


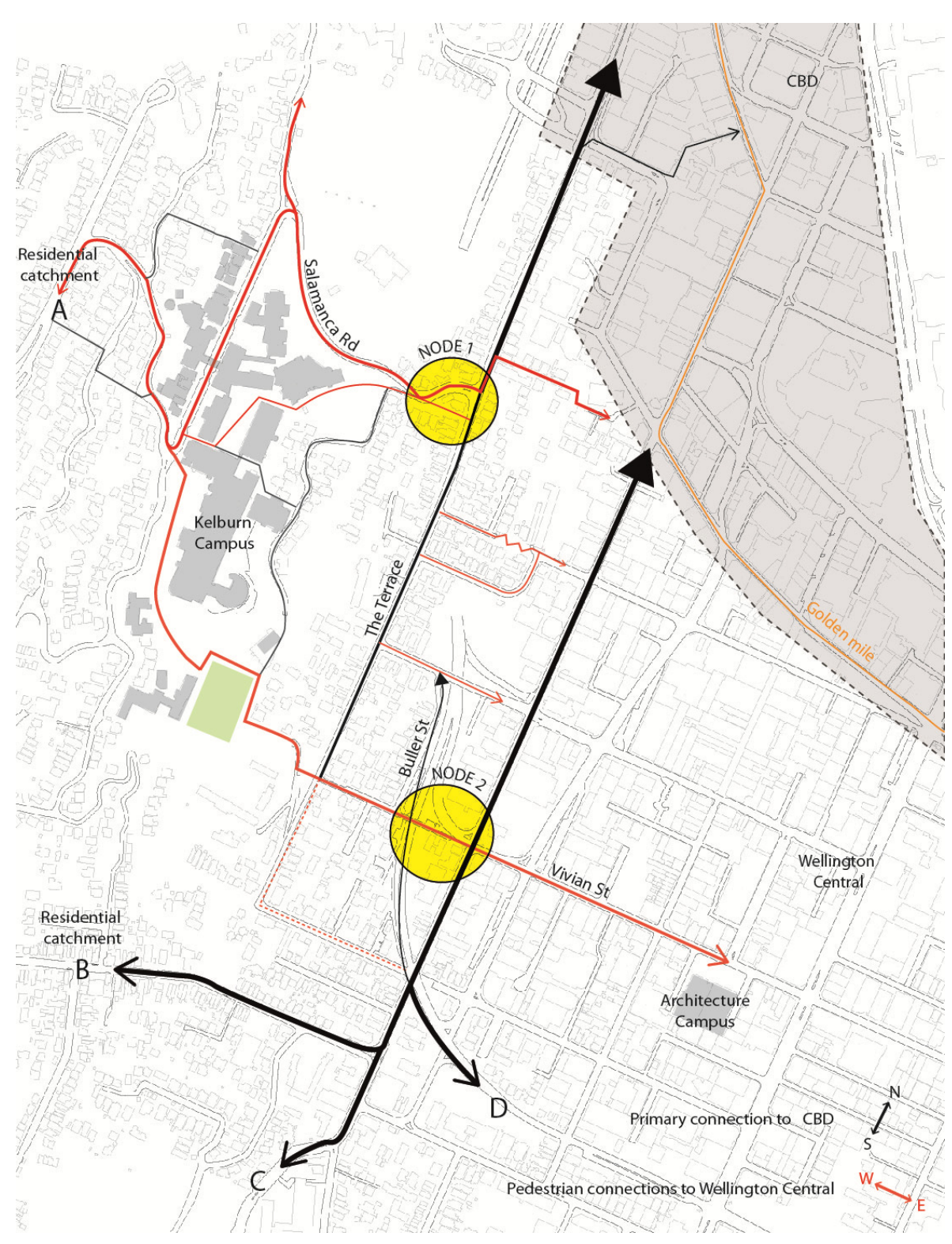

Figure 55: (Macro scale analysis) - Two key nodes in the pedestrian network

\section{Node 1}

Node 1 (Figure 56) is located on a key junction between the Vivian St connection, and the most direct route linking residential catchments $\mathrm{B}, \mathrm{C}$, and $D$, to the University. The site is important because:

- It is located in the centre of the public space void that was highlighted in Figure 54.

- The site marks the edge where residential land-use meets commercial land in the central city area, thus the site has natural surveillance from the surrounding houses. Also, the site is close to a variety of land use types, which means any public space development is more likely to be occupied throughout the day.

- Is at the end of a 'dead end st' which has low vehicle use, and is currently occupied by car parking space that receives significant sunlight hours (Figure 56). Thus there is the potential to develop the parking space into a green space which would revitalise the 'neglected public space area'.

Lastly, there is a vacant lot overlooking the site which has potential to be developed into a mixed use building. This would firstly, provide further residential surveillance overlooking the site. Secondly, a ground floor tenant, such as a dairy, could service the many students commuting between the two campuses. 


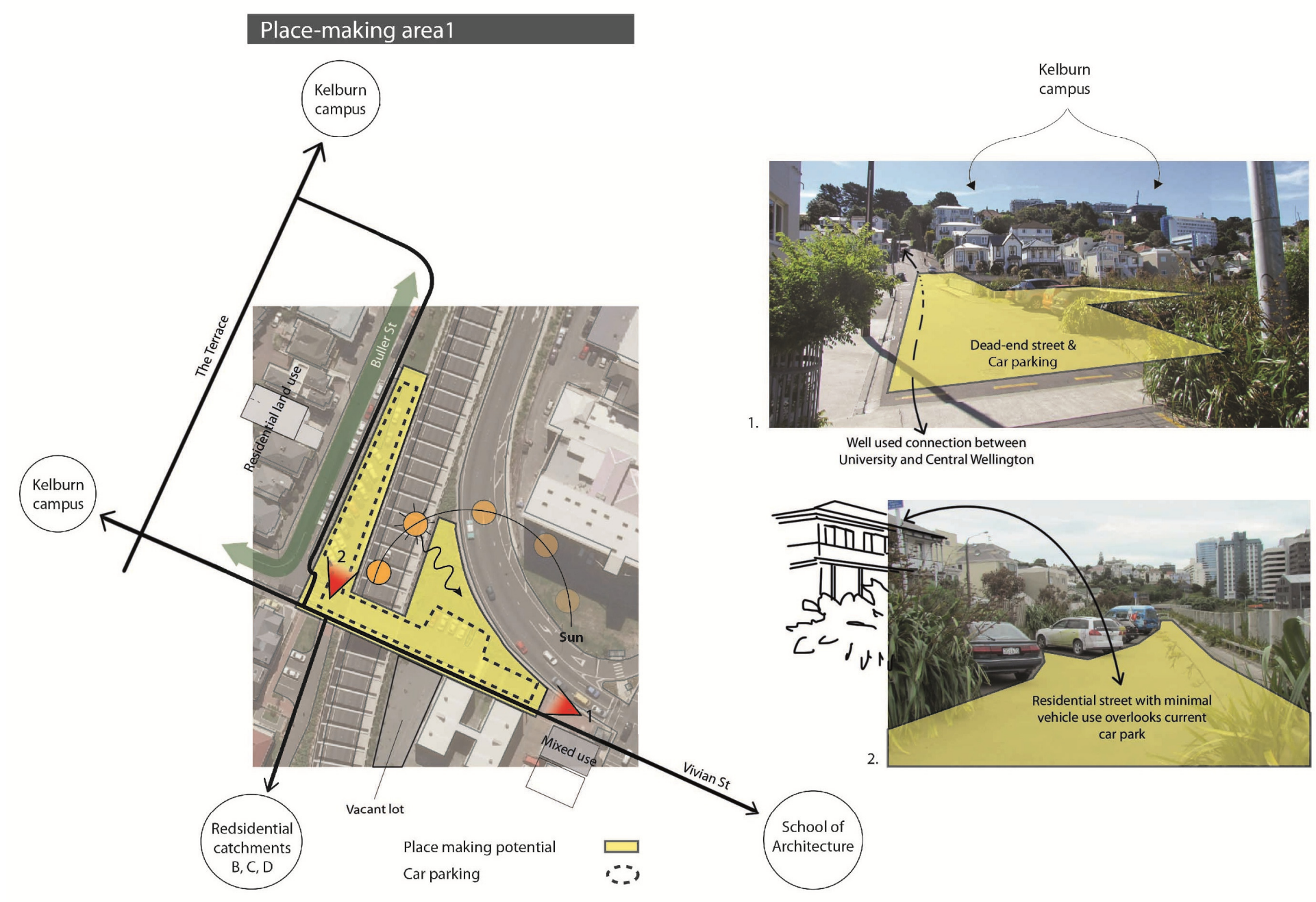

Figure 56: NODE 1 - Place-making qualities 
Node 2

Node 2 is located at the CBD end of Salamanca Rd where it intersects with the Terrace (Figure 54). Note the Terrace is a significant North South pedestrian and vehicle corridor along the Western edge of the CBD. Figure 57 shows the site is important because it:

- Has activity a range of activity from the bus stop, corner dairy, and potentially from surrounding residential houses;

- $\quad$ Receives long sunlight hours;

- Experiences a high volume of pedestrians whom commute to and from residential catchment ' $A$ ', the University Kelburn Campus, and the CBD;

- Has room to be developed as it is set back from the main road.

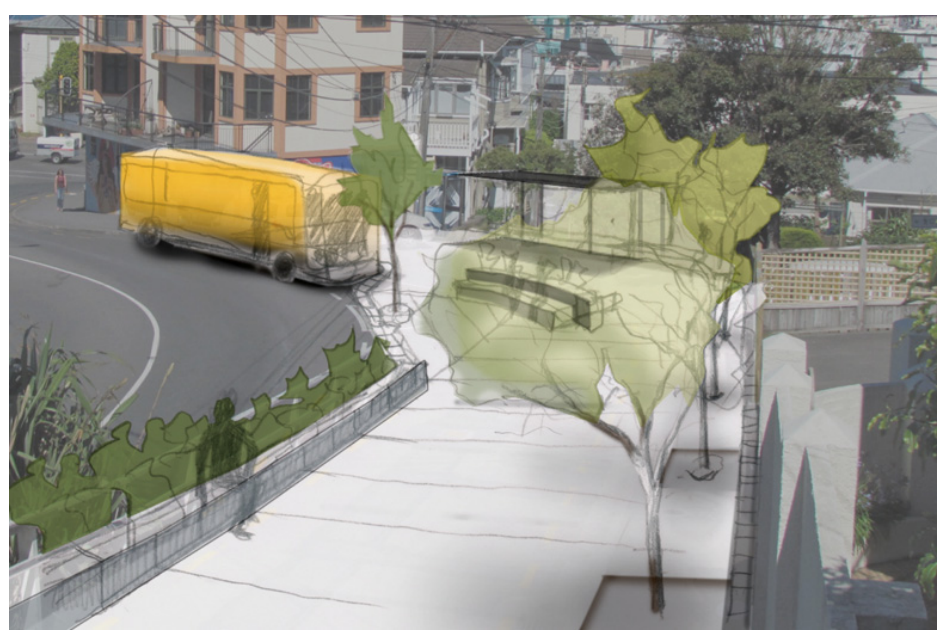

Figure 58: Sample artist impression of new public space
Figure 57

NODE 2

Place-making

qualities

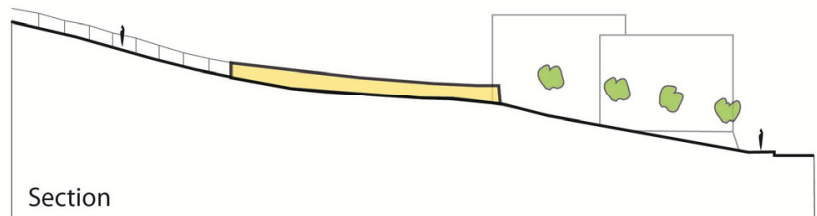

Place making potential

Car parking p

$\therefore$ Bus stop Dairy

(CBD)

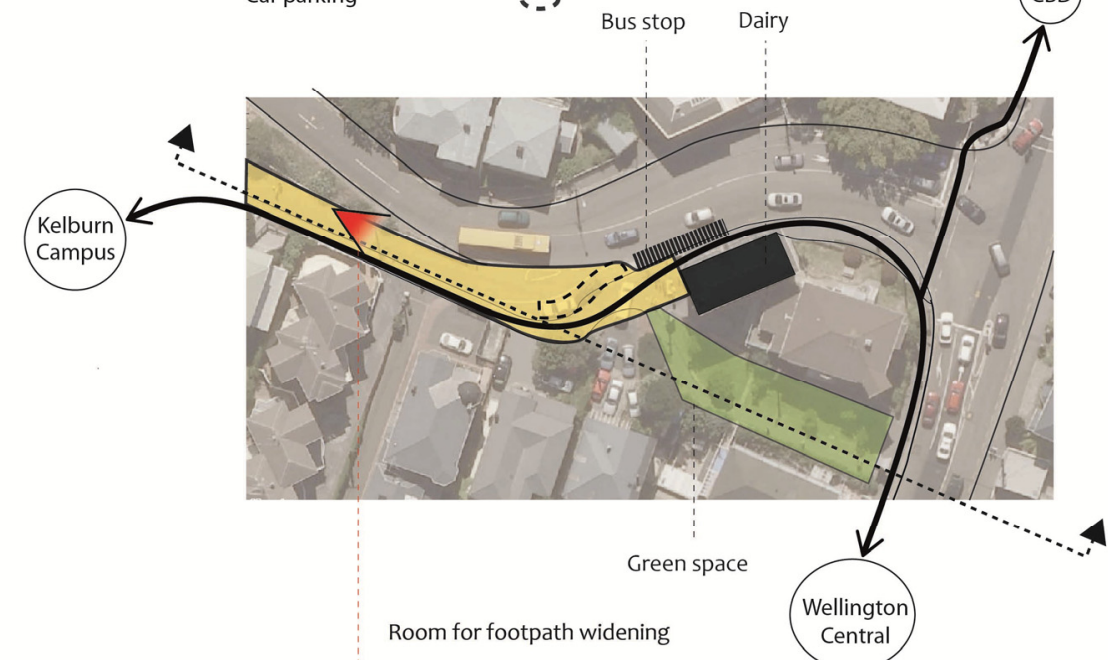

A range of activities meet at this point.

- Well used

corner dairy

- Bus stop - Major pedestrian University and CBD

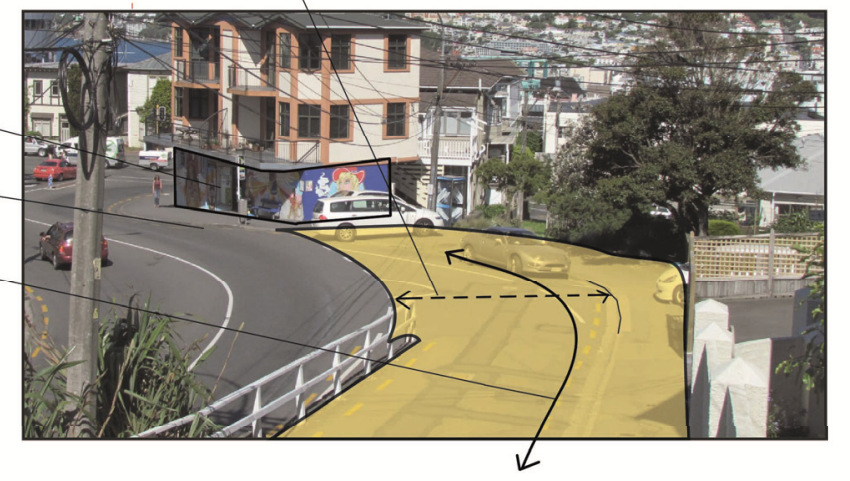




\section{(Micro scale) Place-making analysis and barrier analysis}

The macro scale analysis was useful in understanding how the site sat within the larger pedestrian network. The analysis identified Vivian St and Salamanca $\mathrm{Rd}$ as significant pedestrian connections in the pedestrian network, and also two key nodes (Nodes 1 and 2) as areas of place-making potential.

\section{Step 4}

For the final stage of the site analysis, a micro scale study was carried out to see if there were any areas of place-making potential within the major and minor barrier 'hot spots' A, B, C, D, E, F. The study revealed three new areas, these are highlighted in orange in Figure 59, which is a summary of all the barrier 'hot spots' and areas of place-making potential that were identified within site.

\section{- (Previously identified)}

- (Macro) scale 'top down' analysis of the wider urban context: identified the two place-making NODES '1 \& 2'

- (Micro)scale 'bottom up' cognitive surveys: identified place-making area ' 3 ', and also Barrier Zones A,B,C,D,E,F

\section{- (Newly identified areas of place-making potential)}

- (Micro) scale 'top down' analysis of the barrier hot spots: identified place-making sites, 'area 3, 5, \& 6'. Note these three areas are discussed shortly.

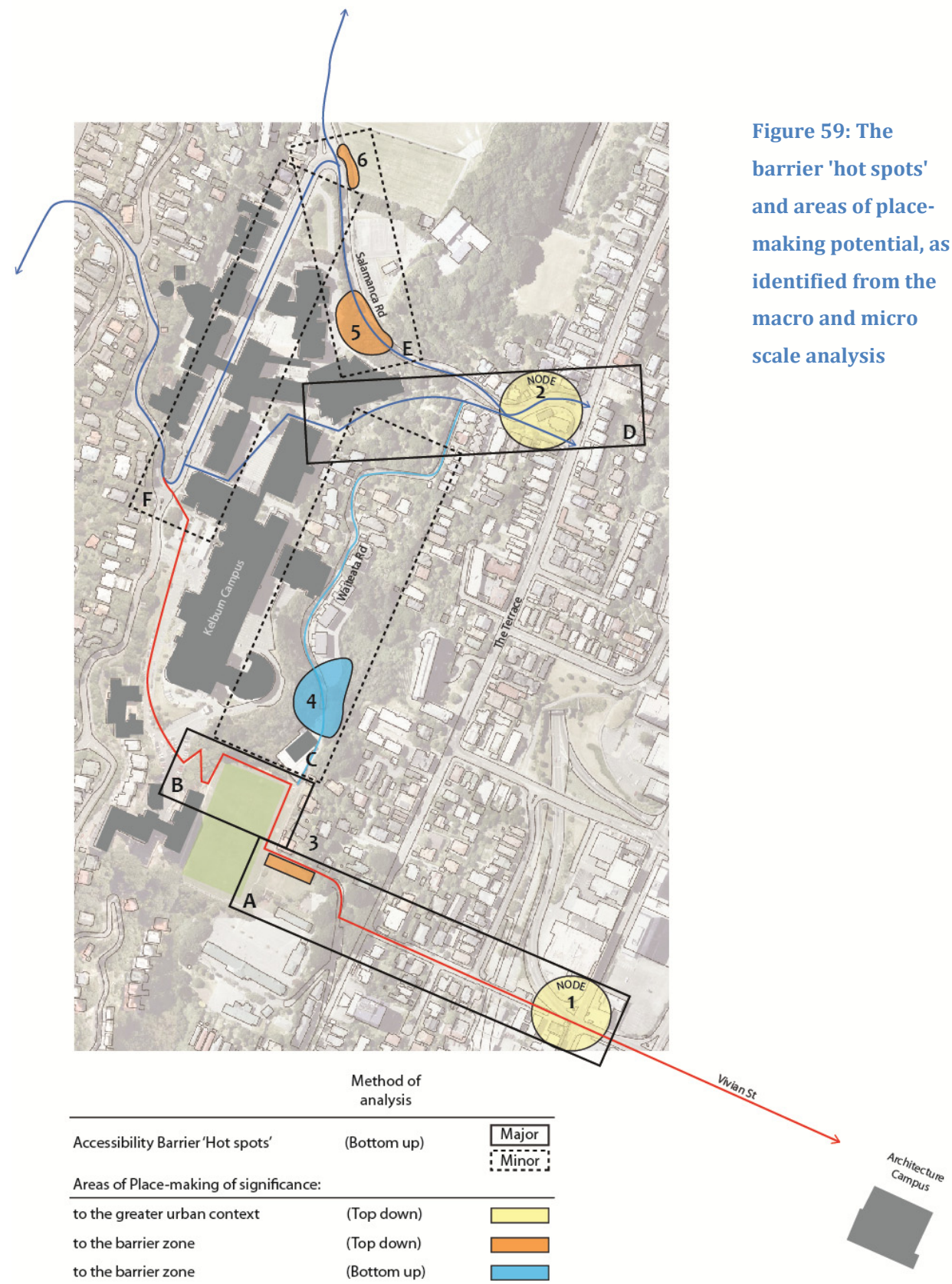




\section{Forming a response to the site analysis}

The purpose of this section was to trial the following two design approaches together as a cost efficient and effective approach for both resolving accessibility barriers, and developing areas of place-making within site:

- The 'customised place-making solution', which responds sensitively to the surrounding environment whilst capitalising on areas of untapped place-making potential;

- The 'standardised typological solution, which resolves the accessibility barrier in the most low cost and efficient manner,

\section{Forming a Cost Effective Strategy}

A hierarchical framework (Figure 60) was designed to help ensure the design and implementation process, for resolving the accessibility barriers and developing the areas of place-making potential on site, was cost efficient. The framework was designed by ordering into a hierarchy of urgency the main scenarios that could arise when designing pedestrian infrastructure. This was done by ranking each scenario against the following key issues, which in order of importance were:

1. Cost;

2. Resolving pedestrian accessibility barriers;
3. Capitalising on areas of place-making significance in the pedestrian network.

The framework ranks each of the scenarios by their cost versus their urgency to be addressed (either Stage 1, 2, 3, or 4). This is significant, because it provides a system for implementing multiple pedestrian infrastructural improvements in a network, in the most cost efficient manner. The following example will explain how the framework works by reading across the first scenario in the table.

If a site has been identified as having a significant accessibility barrier present, as well as place-making potential; there are two possible design options authorities can implement.

1. If the site is of stage 1- (high) urgency to address. In this scenario the proposed 'temporary solution' has a lower cost; however, as a consequence there is no improvement to the quality of public space.

2. Alternatively, if the site is graded of stage 2- (mid-high) urgency to address. In this scenario using the proposed long-term solution has a slightly larger cost; however, it results in a high level of improvement to the quality of public space.

There are two things to note about this framework: 


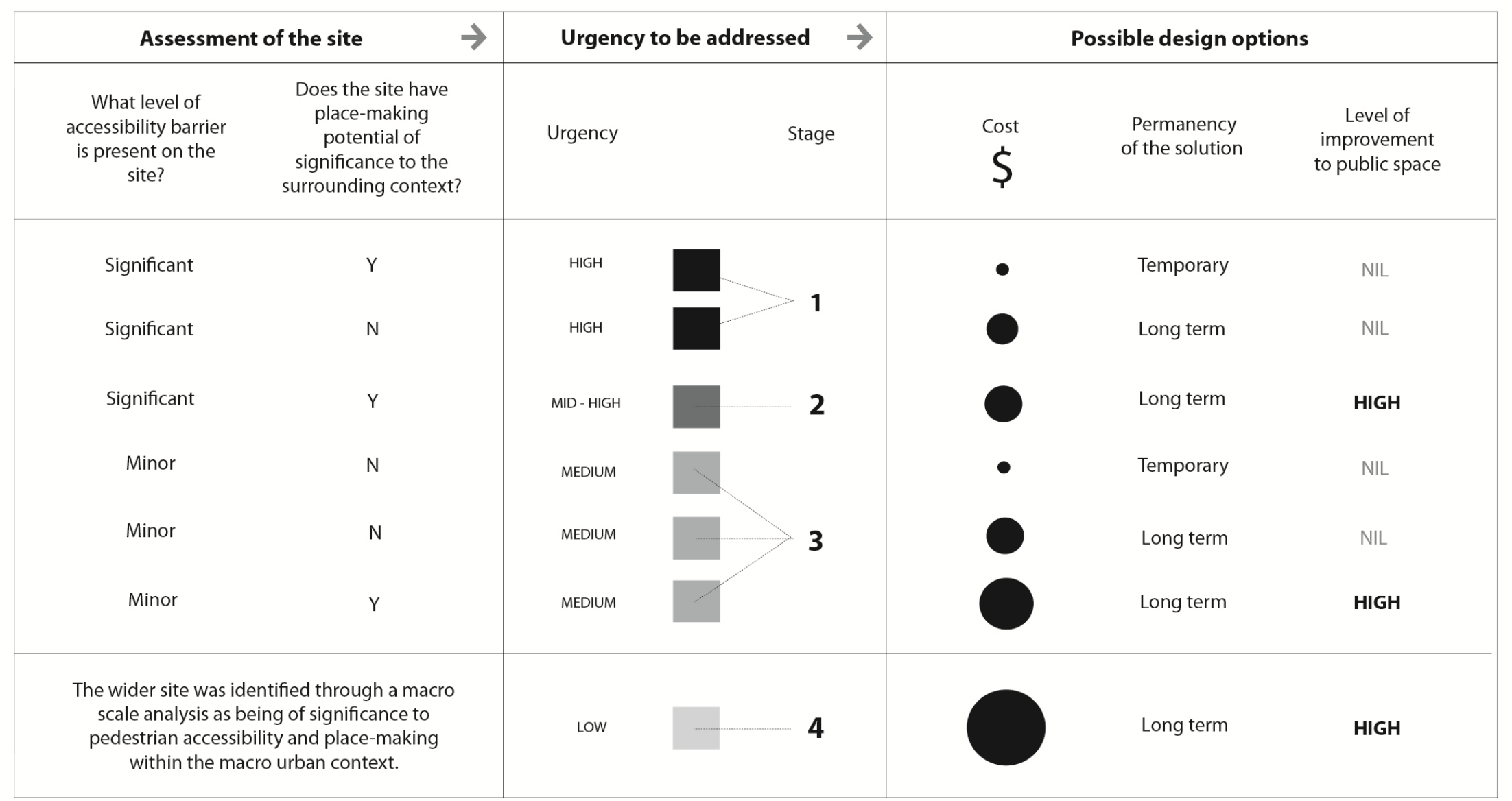

Figure 60: Hierarchical framework for implementing pedestrian infrastructure. Cross referencing - Site conditions / Cost / and Urgency

- Firstly, in principle the solution for a design that resolves a minor barrier is considered to cost less than a solution that resolves a

significant barrier, when neither design 'incorporate place-making'. This is because it is assumed that resolving minor barriers require a less drastic response to site and therefore can be done at a lower cost.
- Secondly, this framework is only a guide, as in practice the cost of the solution will vary between the type of barrier and the amount of resources required for the intervention. For example re-paving an entire walkway may cost more than adding more lighting. 


\section{Designing the Interventions}

Note for the remainder of this chapter the fold out the leaflet attached to the inside of the back cover will be referred to.

Using the new hierarchical framework (Figure 60), this section will now explain the process that was undergone to:

- $\quad$ Resolve each of the minor and major 'barrier hot spots' within the sites 'Barrier Zones A, B, C, D, E, F' (foldout leaflet).

- Whist also capitalise on the six areas of site that were identified as having place-making potential (areas 1, 2, 3, 4, 5, 6, on the foldout leaflet).

\section{Barrier 'Hot Spot' Zone (A): Eastern end}

Site Analysis

Figure 61 identifies that the cognitive surveys highlighted two accessibility barriers along the Eastern end of 'Barrier Zone A'. These were relating to:

- $\quad$ Comfort $=$ no shelter from weather

- $\quad$ Path Quality = steep gradient, and slippery footpath surface.

These barriers were spread across two access typologies, the 'Pedestrian over bridge' and 'Park Field' (Appendix A, pg204 and 307), and also one land use typology 'Urban Residential St' (Figure $15 p$ 57). Note here that the area identified within site as a Path Field currently is a car park; however it has been labelled as a Park Field because it was identified in
Figure 55 pg132 as a key node with the potential to become a new park space.

To begin with, the typological analysis was applied to site to identify the significant spatial conditions of each accessibility barrier typology that needed to be considered when designing a barrier response. These spatial conditions were:

\section{Urban Residential St}

- $\quad$ The design must avoid creating visual obstacles that prevent eyes on the street, and block the views to interesting houses. Objects such as high property fences/planting can prevent interaction between pedestrians and neighbouring properties.

\section{Over Bridge}

- $\quad$ The design should avoid creating tall balustrades/bridge walls as they cause users on elevated bridges to be hidden from view.

\section{Park Field}

- The design should avoid creating built or landscaping vegetative features that cause hidden and potentially dangerous secluded areas.

- The design should be mindful that poor drainage on grass areas can cause footpaths to be flooded under extreme weather.

As a result the concept Figure 62 was designed. 

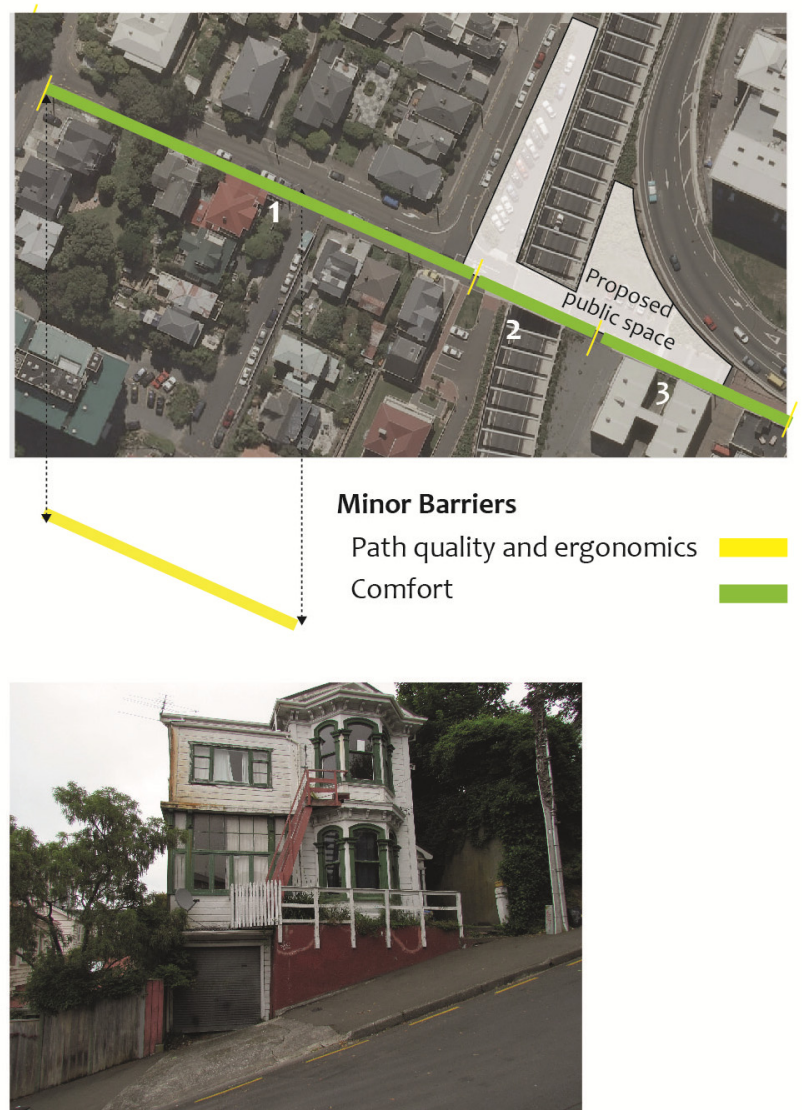

The aesthetic quality of residential houses needs to be considered along path section (1)

Figure 61: Barrier Zone 'A' accessibility barriers and consideration - Eastern end

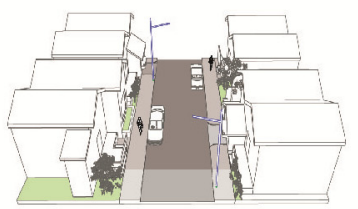

Comfort

No shelter from weather

Path quality and ergonomics

- Slippery

- Too steep

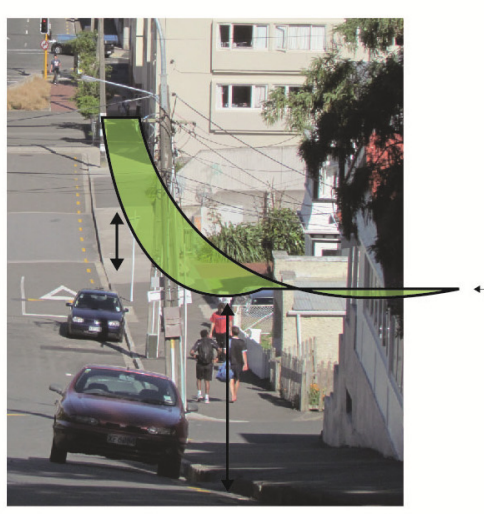

Comfort

No shelter from weather

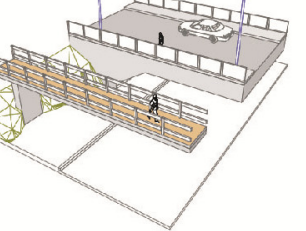

Comfort

- No shelter from weather

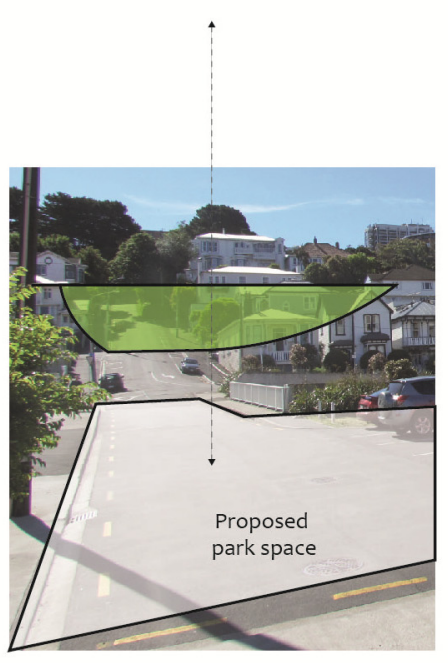

Designing shelter through the park space requires less aesthetic

consideration of context as no

houses are immediately present. 


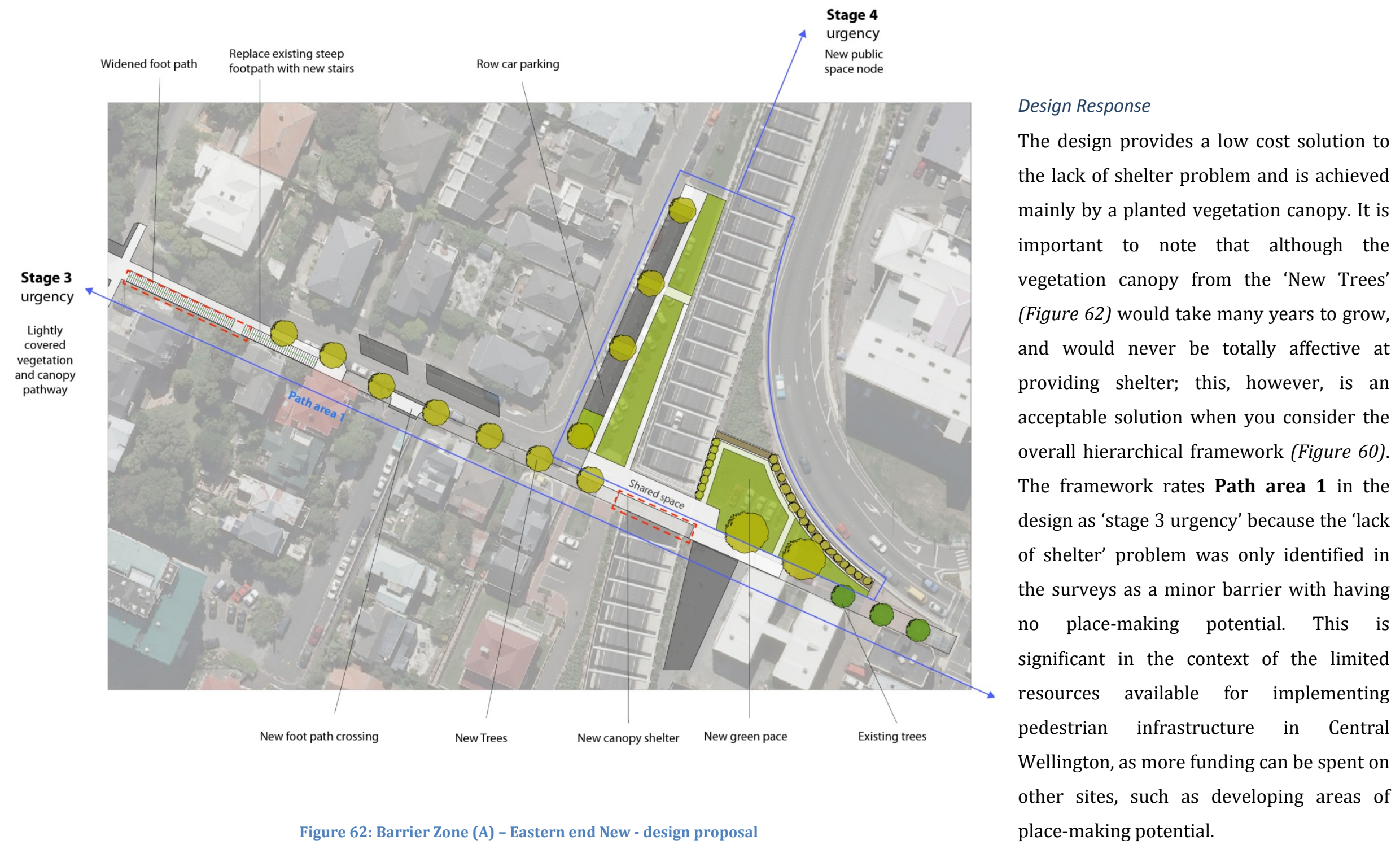




\section{Site Analysis}

To begin with the typological analysis was used to understand more about the accessibility barriers present at the Western end of 'Barrier Zone A'. The minor barriers present in this area of site were (Figure 63):

- $\quad$ Comfort $=$ No shelter from weather

- $\quad$ Path Quality = Slippery footpath surface

The significant barriers present were (Figure

64):

- $\quad C P T E D=$

- Insufficient lighting

- Enclosed space

- Hidden pockets off the footpath

- $\quad$ No eyes on the street

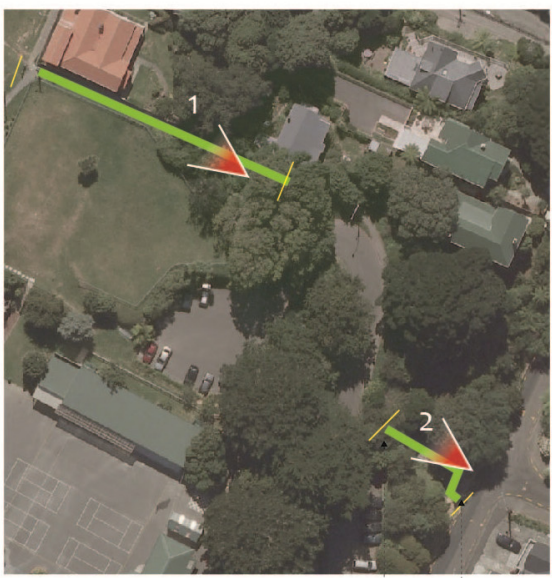

1

2

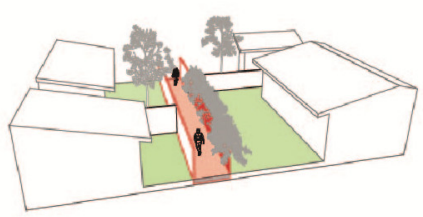

Comfort

- No shelter from weather
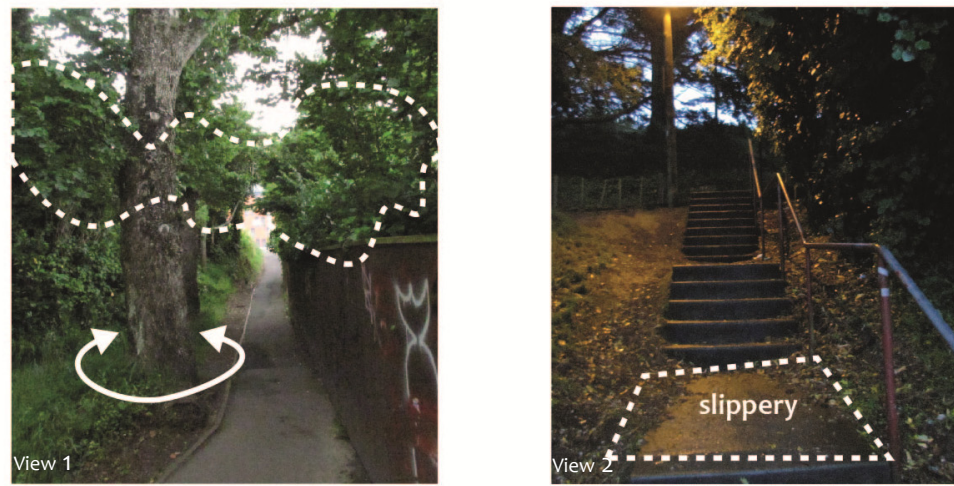

Some shelter from trees however trunks and branches create blind spots.

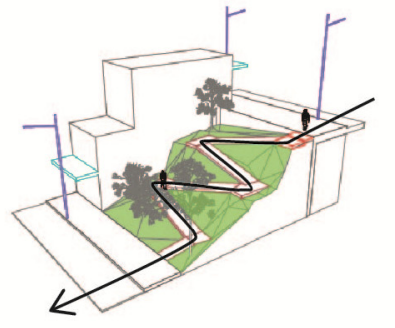

Path quality and ergonomics - Slippery footpath

\section{Minor Barriers}

Path quality and ergonomics

Comfort : No shelter from weather 


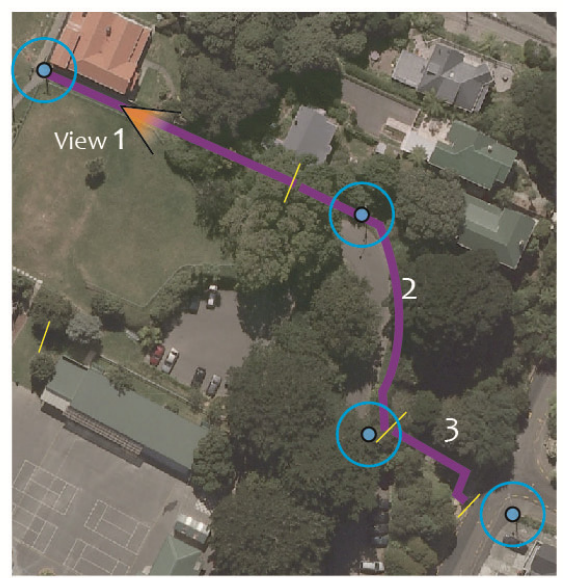

CPTED Lighting and Spatial Existing lighting
1

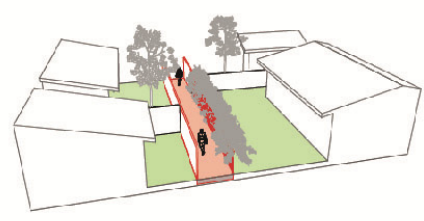

CPTED Lighting and Spatial

- In sufficient lighting

- Enclosed space

- Hidden pockets off footpath

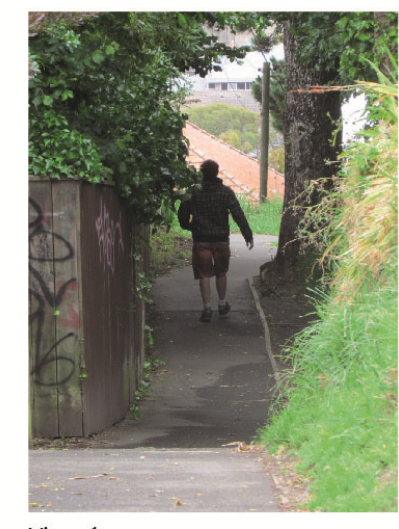

View 1
2

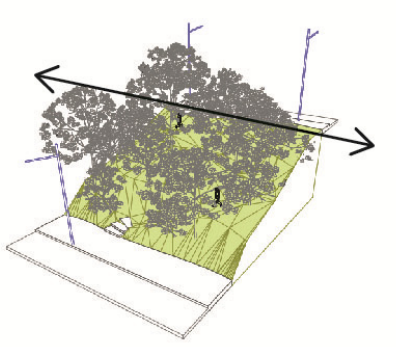

CPTED Lighting and Spatial

- In sufficient lighting

- No eyes on the street

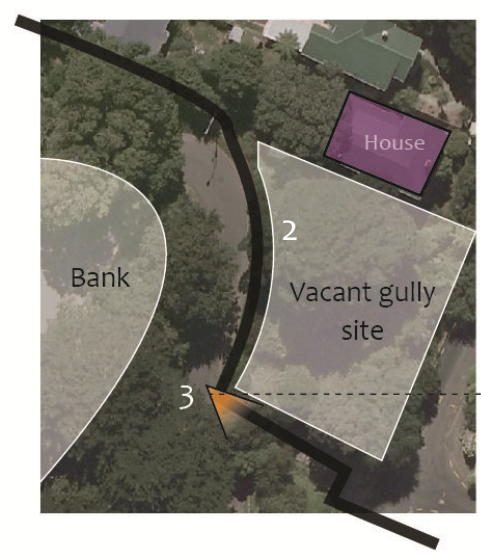

path with no surveillance
3

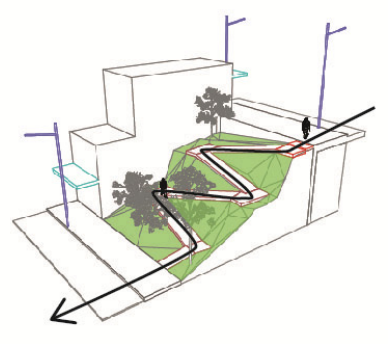

CPTED Lighting and Spatial

- In sufficient lighting

- Enclosed space

- Hidden pockets off footpath

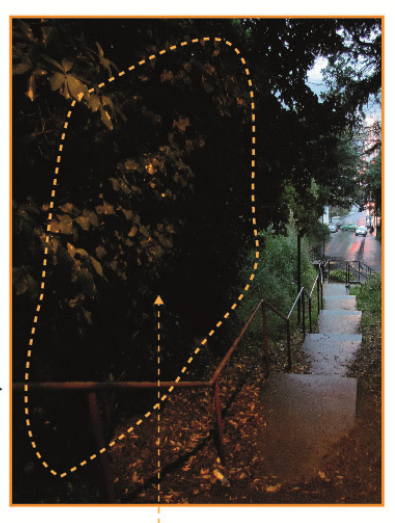

Hidden pockets 
Next the typological study was used to identify the important spatial conditions, which were associated with each barrier typology on site, that need to be considered in the design response. This was to prevent the design response from accidentally creating new barriers. The additional spatial conditions were:

- $\quad$ 1. Passageway Residential typology (Figure 14 pg56)

- Bends along the path and impressions in the fence walls can create secluded alcoves.

- Narrow and single entry exit points offer few escape routes.

- High hedges and fences can prevent 'eyes on the street'

- $\quad$ 2. Vegetation Gully typology (Appendix pg212)

- Dense foliage creates hidden areas off the path, and can also be a visual barrier preventing surveillance from the surrounding buildings.

- $\quad$ Large changes in typography can also interrupt surveillance from surrounding buildings.

- $\quad$ 3. Terrace path typology (Appendix pg196)

- Users can become hidden from the view of the main street, particularly if dense vegetation is prominent. Then when on the path, bends and vegetation can create hidden blind corners further ahead.
In addition to these barriers, one area of place-making potential, 'Placemaking area 3', was identified within 'Barrier Zone A' (see Figure 65). The place-making qualities of the area were:

- Receives long sunlight hours,

- Is located off a well used pedestrian thoroughfare, for students walking between Kelburn University campus and Architecture campus.

- Is close to the university sports centre and Te Puni hostel.

- The site required significant changes as there were a number of barriers present within it. Thus there is an opportunity to easily integrate a public space design into the new changes.

In response to these points the concept shown in Figures 66-67 was designed: 

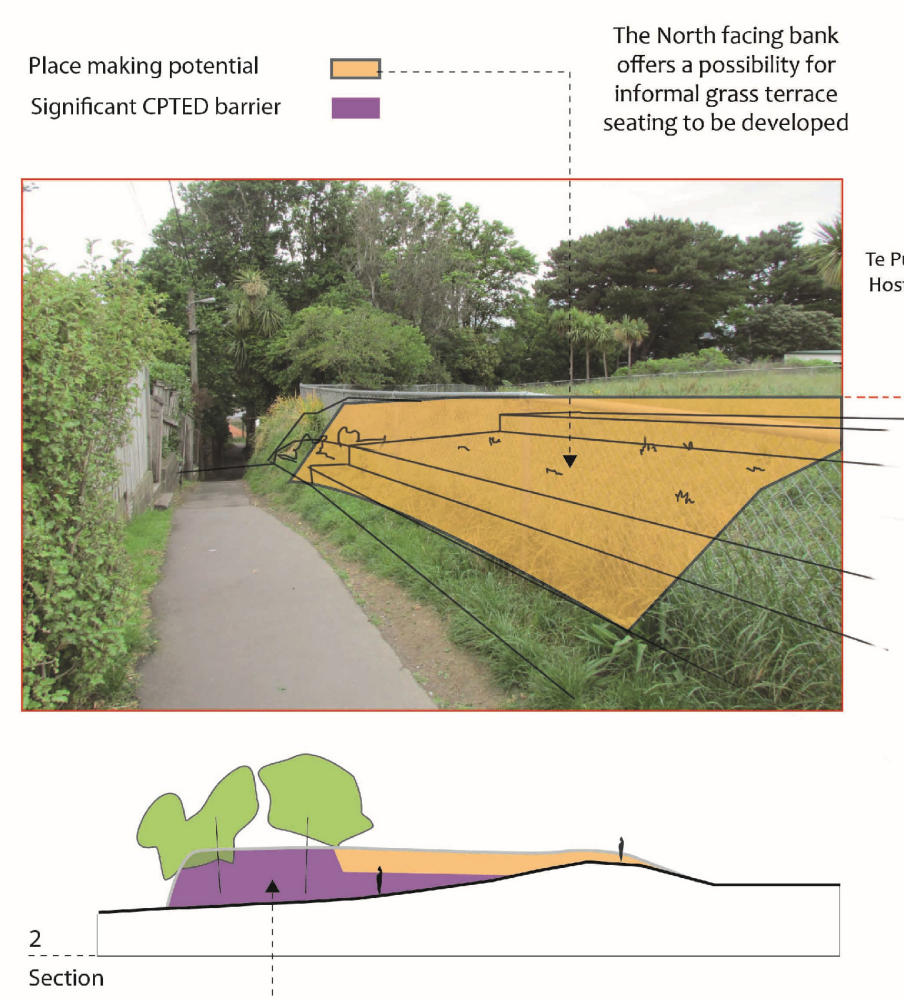

High' bank CPTED barrie
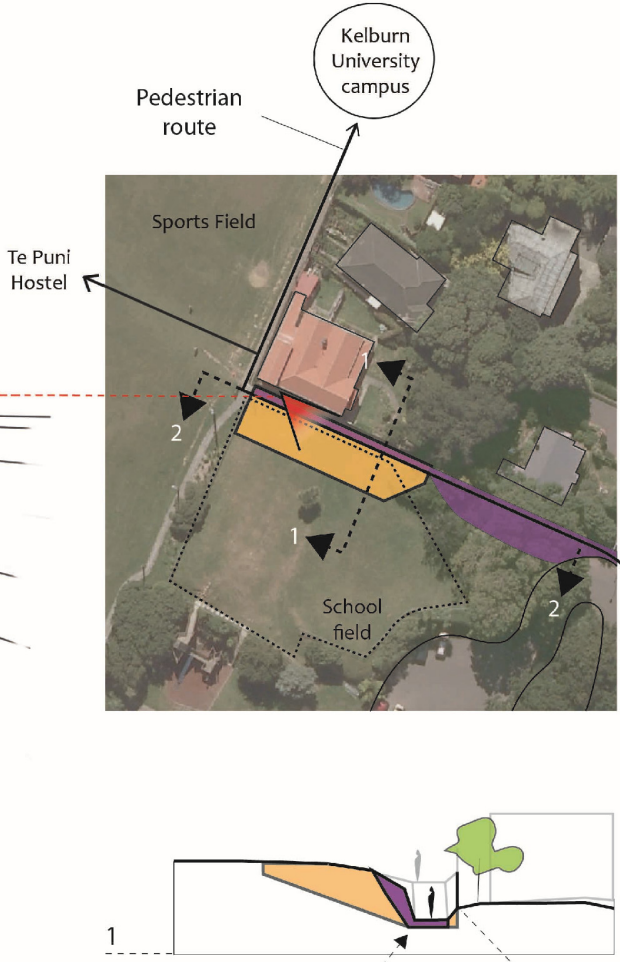

Narrow path CPTED barrier

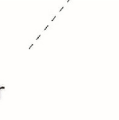

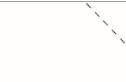

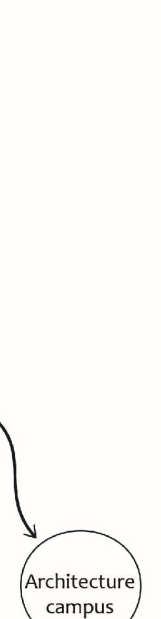

An opportunity for placemaking is created due to the significant changes that are

required to excavate the

order to resolve the CPTED barriers

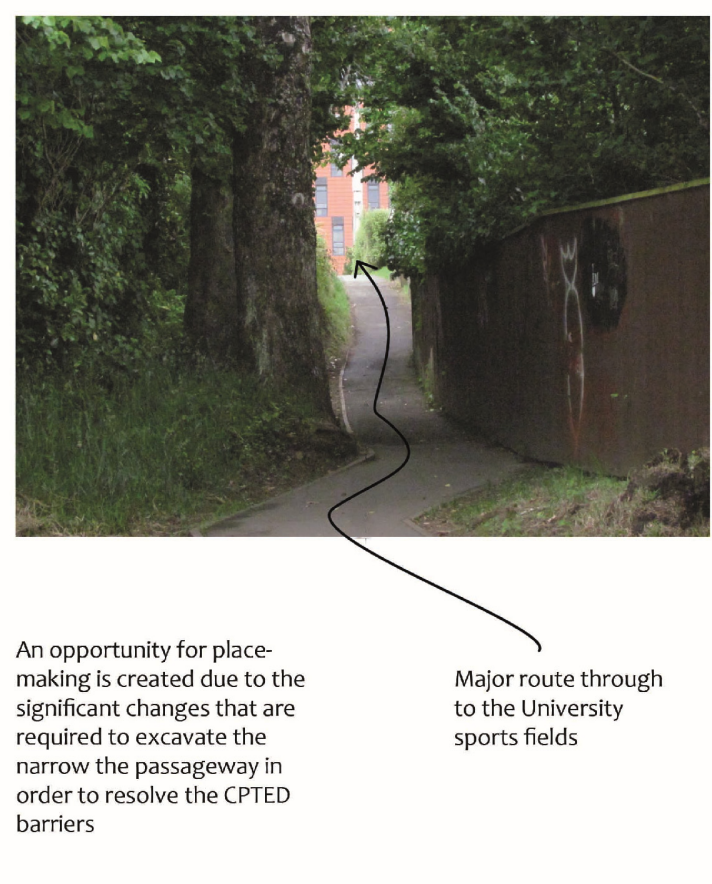




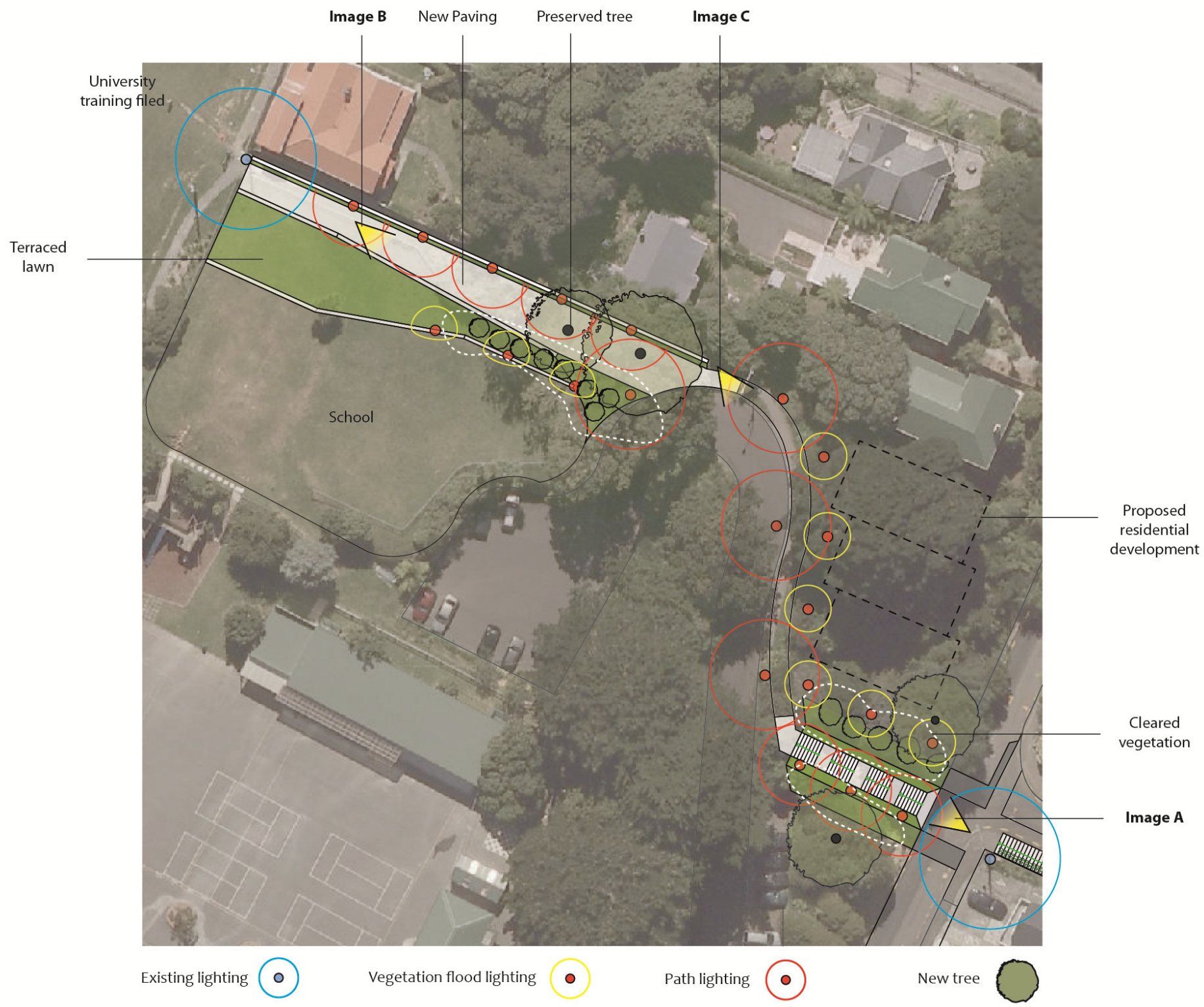

Figure 66: Barrier Zone (A) - Western end - Proposed solution 


\section{Design Response}

The design successfully resolves the major CPTED barriers by:

- Addressing the 'hidden areas off the footpath', and 'lack of lighting', through a new lighting strategy that illuminates areas both off and on the footpath (Image A).

- Providing clear safety sightlines between major entry and exit points (Image B).

- Identifying a key vacant site to be developed into residential land use, this as a long-term strategy will generate more surveillance through improved 'eyes on the street'. Note such developments could be fast tracked through incentives funded by the Local or Central government i.e. reduced property rates (Image $\mathrm{C}$ ).
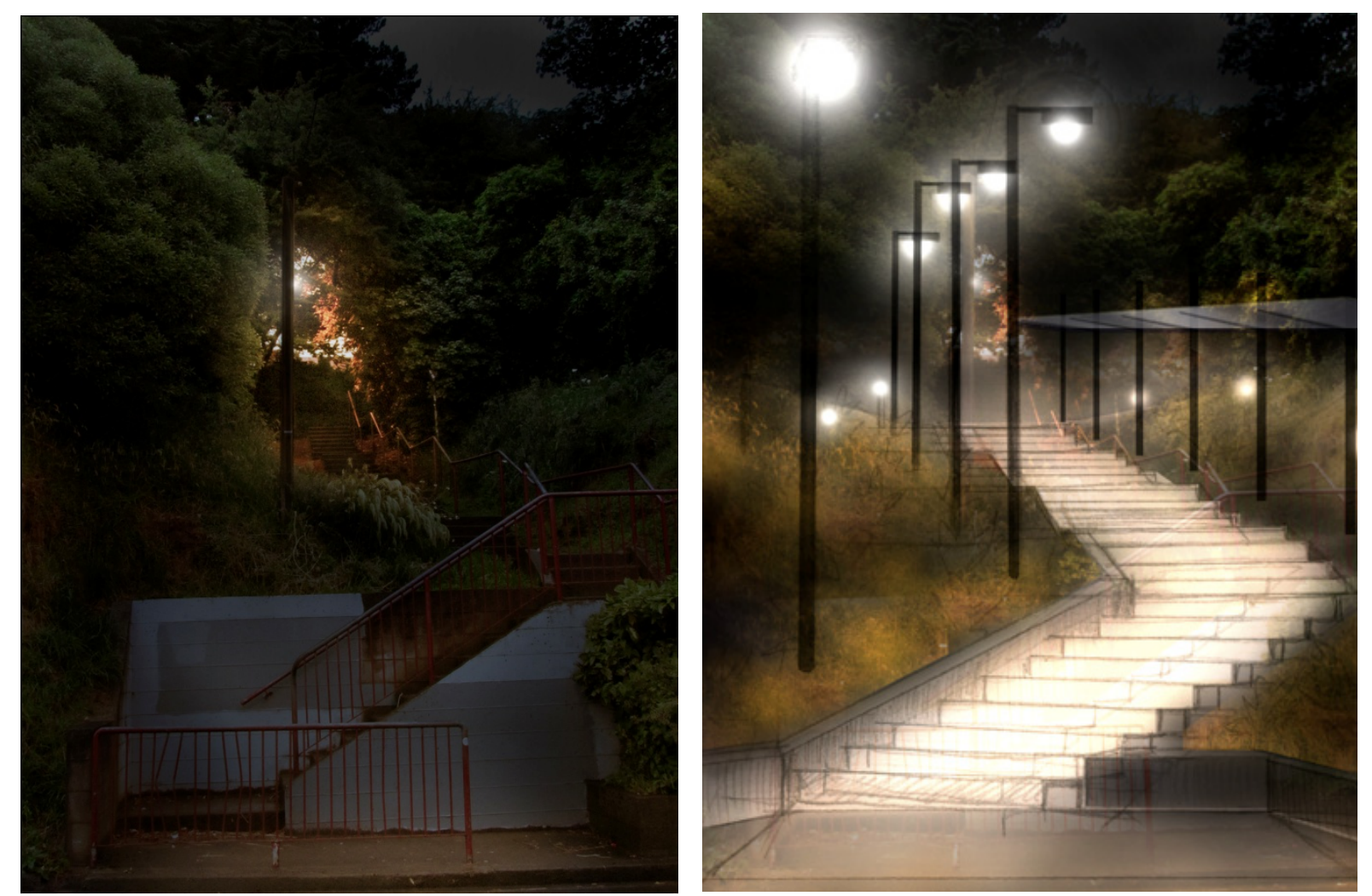

Image A

Figure 67: Artist impressions before -> afters 

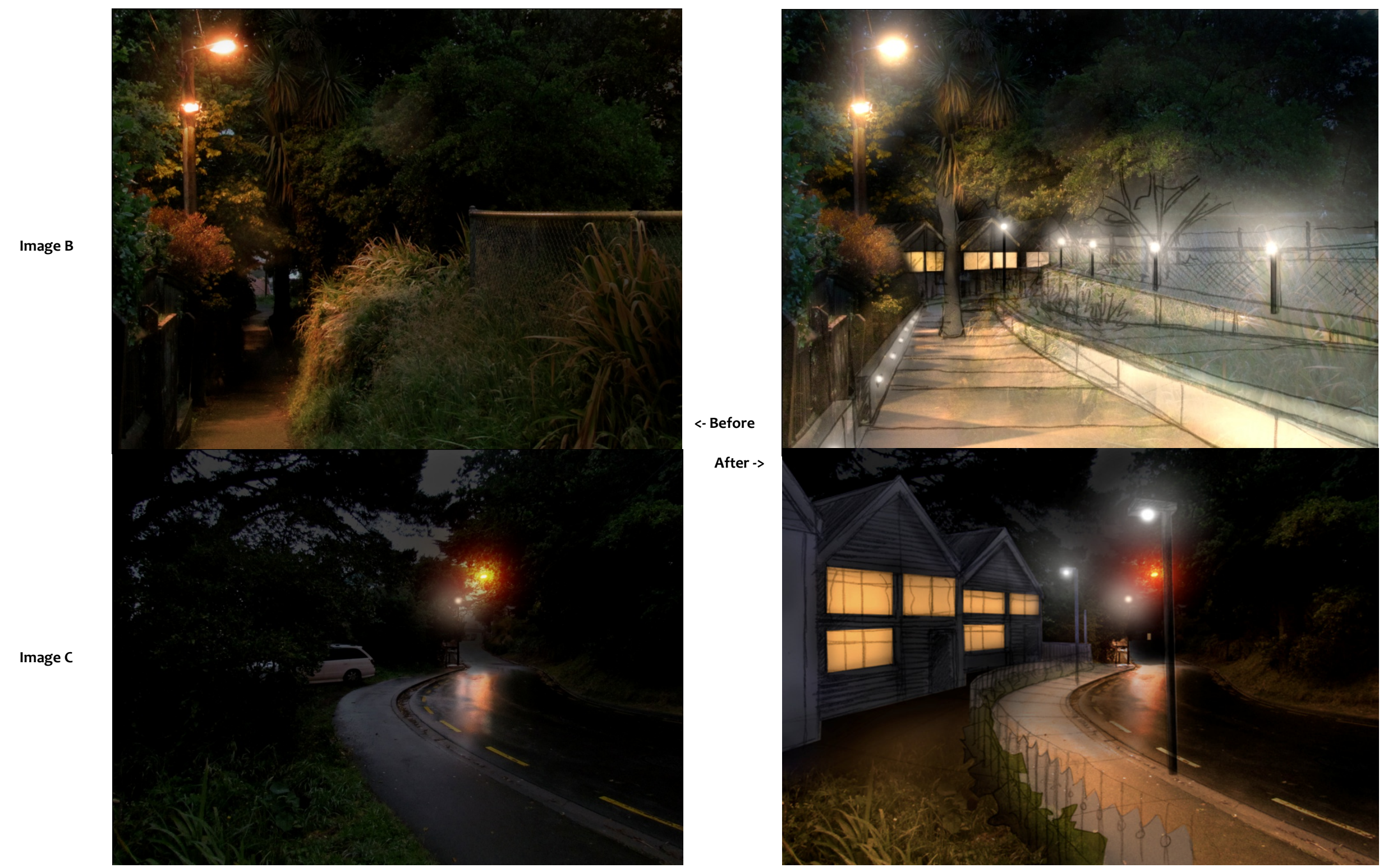
In terms of the new public space design's contribution to place-making in the pedestrian network, the design offers a North facing terraced lawn that could allow for informal grass seating. This is an activity that the other adjacent fields cannot provide because the primary school field is occupied by the school most days of the week, and the University playing field is less appropriate to sit on because the artificial rubber surface becomes heated during the direct sunlight, and the field is often fully occupied for sports training.

Regarding the minor barriers of 'no shelter from weather' and 'slippery path surface', these barriers are of 'stage 3 urgency' in the overall hierarchical framework (Figure 60 p137), thus do not need to be addressed immediately. Therefore if not enough resources were available to implement the permanent solution proposed in Image A, a lower cost temporary solution could be to:

- Address the minor barrier of 'slippery path surface' simply by attaching a metal grate/grip to the stair treads.

- Add more lighting and cut back or remove the dense overgrown vegetation, as a way of resolving the major CPTED barriers of 'lack of lighting on the footpath' and 'lack of visibility off the path.

- Delay implementing even a temporary solution for the minor 'lack of shelter' barrier until there is enough funding to build a permanent solution. This is considering the cost of providing evening a temporary shelter could be expensive.

\section{Barrier 'Hot Spot' Zone B}

Site Analysis

Figure 68 shows that the cognitive surveys highlighted two accessibility barriers in 'Barrier Zone B'. The major barrier was:

- $\quad$ Path Quality and Ergonomics = Puddling, and Slippery surface.

And the minor barriers were:

- $\quad C P T E D=$ Lack of lighting causing dark areas off the footpath

- $\quad$ Comfort $=$ No shelter from weather

In terms of the typological study, the relevant spatial conditions of each barrier typology that needed to be considered in the design response were:

- $1 \& 2$. Bush thoroughfare

- The design intervention should be respectful towards the strong presence of natural vegetation.

- 2\&3. Thoroughfare Field

- Built landscaping features, and dense vegetation can create hidden or secluded areas.

Figures 69 and 70 show the proposed design response. 


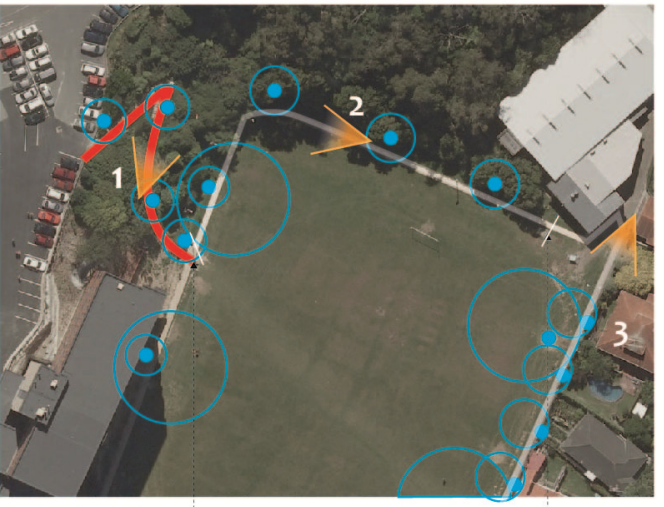

Lighting

- Existing lighting

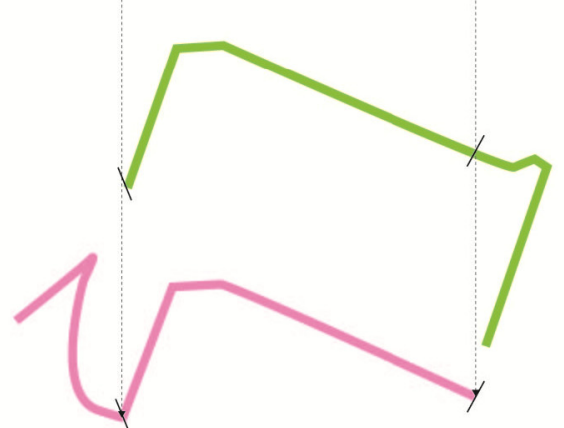

Major

Path quality Ergonomics - Puddling and slippery surface

Minor

Comfort

- No shealter from weathe

CPTED Lighting \& Spatia

- Dark areas off the path
$1 \& 2$

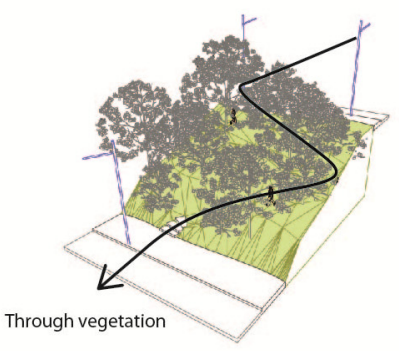

CPTED Lighting \& Spatia

- Dark areas off the path

\section{Path quality / Ergonomics}

- Puddling and Slippery surface
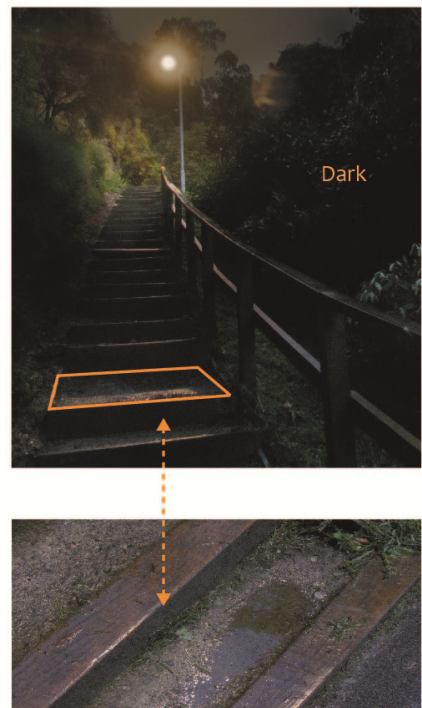

$2 \& 3$

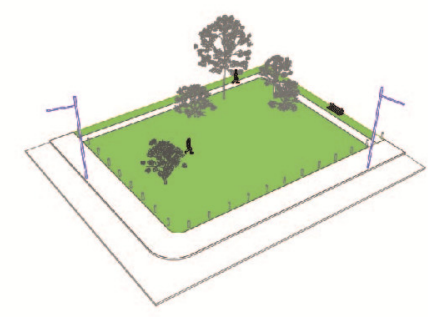

Comfort

No shelter from weather

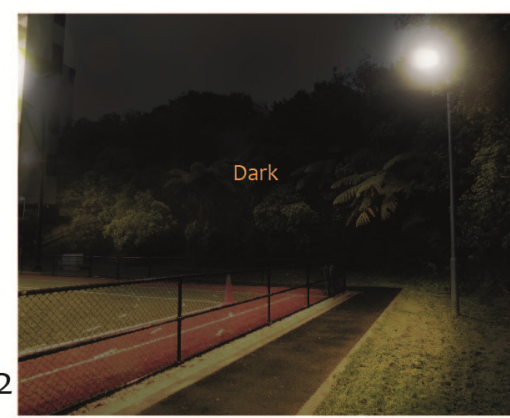

exposed to weather

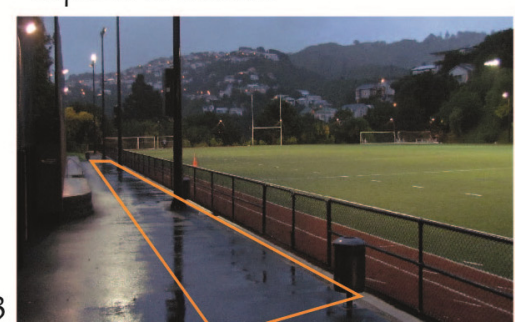

Figure 68: Barrier Zone

(B) - Minor \& Major

accessibility barriers and considerations 


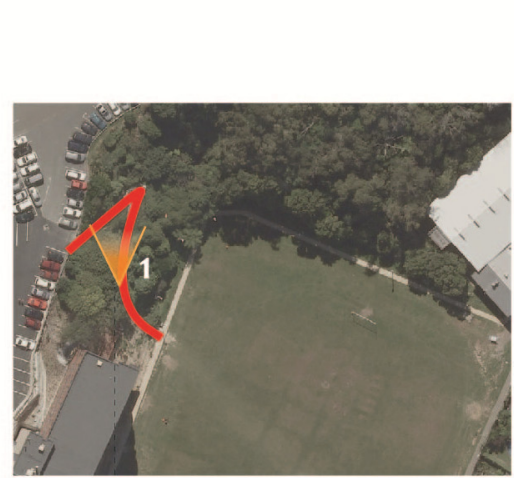

Problem area

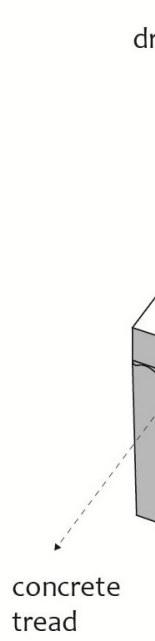

$$
\text { tread }
$$

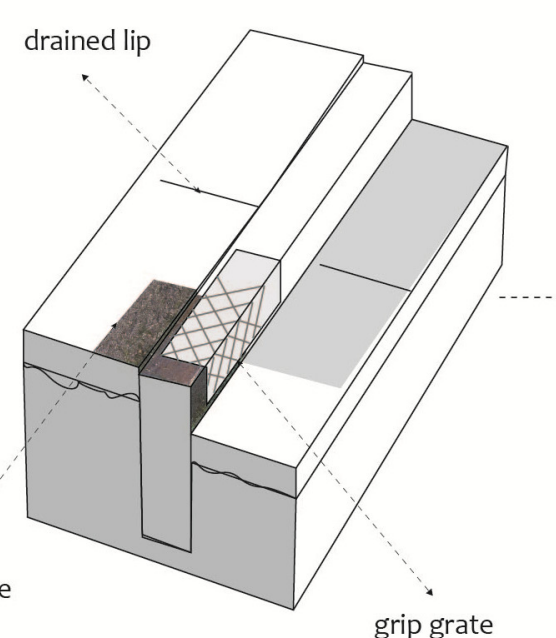

grip grate

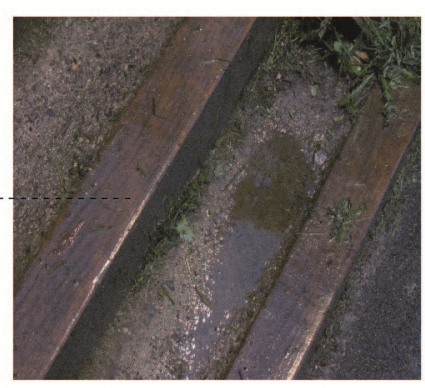

Figure 69: Barrier zone (B) Retrofit to existing stair treds

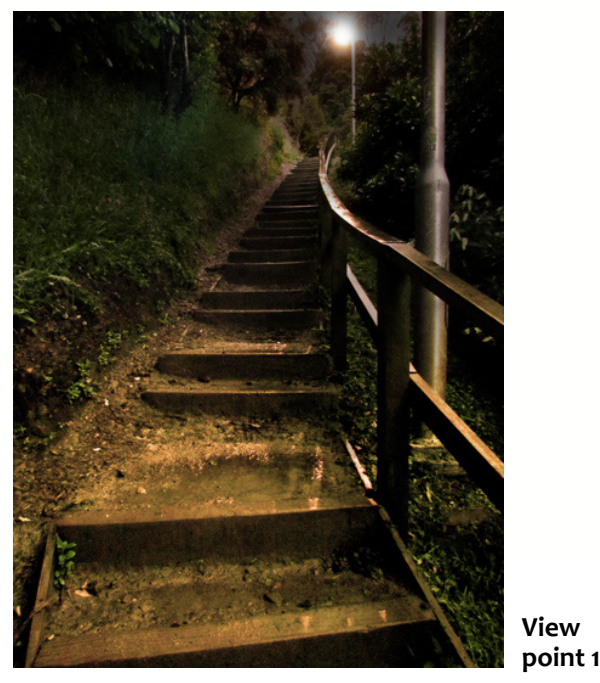

<- Before After ->

View

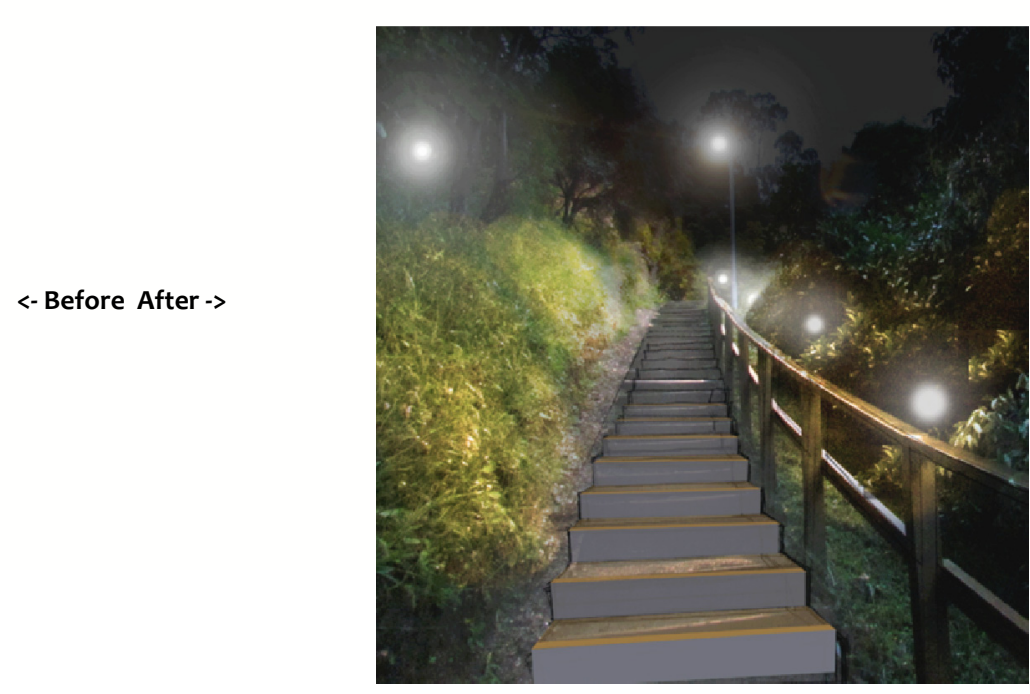

\section{Design Response}

In the design intervention the major barriers of 'puddling' and 'slippery footpath surface' were resolved by retrofitting the existing aggregate stairs treads with concrete (Figure 69). This is a response that could easily become a standardised typological solution to this typology's problem.

The minor barriers of 'insufficient lighting off the footpath' and 'no shelter from weather' were resolved through the standardised typological solutions for a canopy shelter, and vegetation lighting (Figure 70). Note the decision to use a typological solution for the canopy was because there were no significant place-making characteristics in the surrounding context that needed to be considered; apart from the frequent activity on the sports field that raised the possibility of integrating a small seating area in to the design (View point 2). 


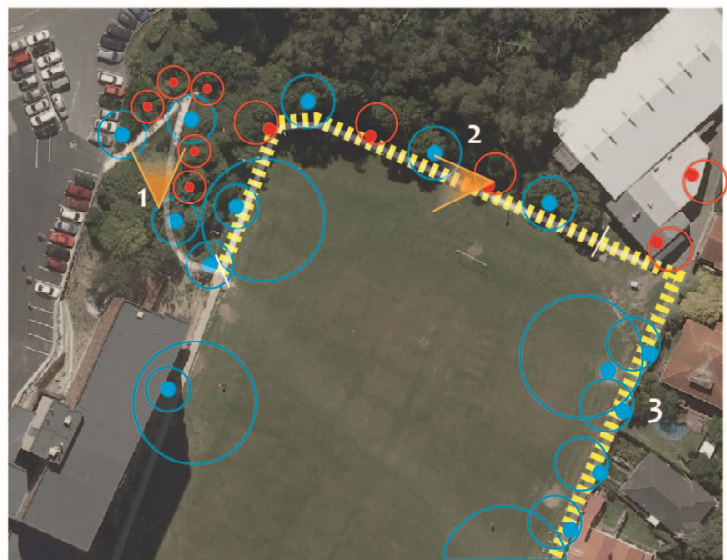

\section{Lighting}

Existing

New shelter IIII!

Proposed additional

$\odot$

$1 \& 2$

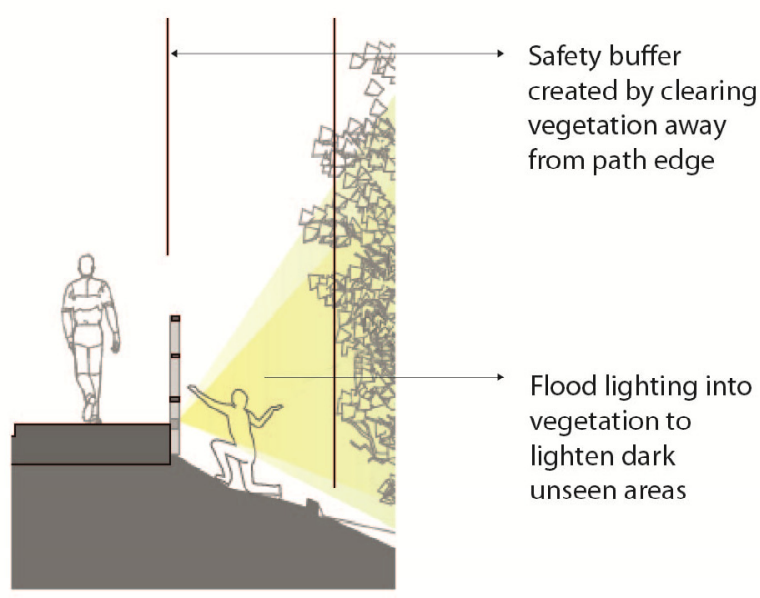

$2 \& 3$

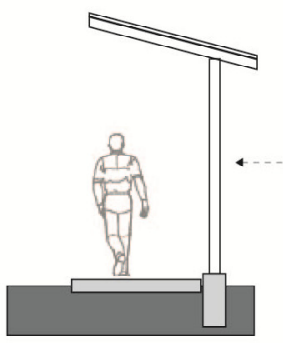

Steel materiality from existing site lighting and fences is applied

to the shelter intervention.

Figure 70: Barrier zone (B) - Standardised typological lighting and shelter improvements

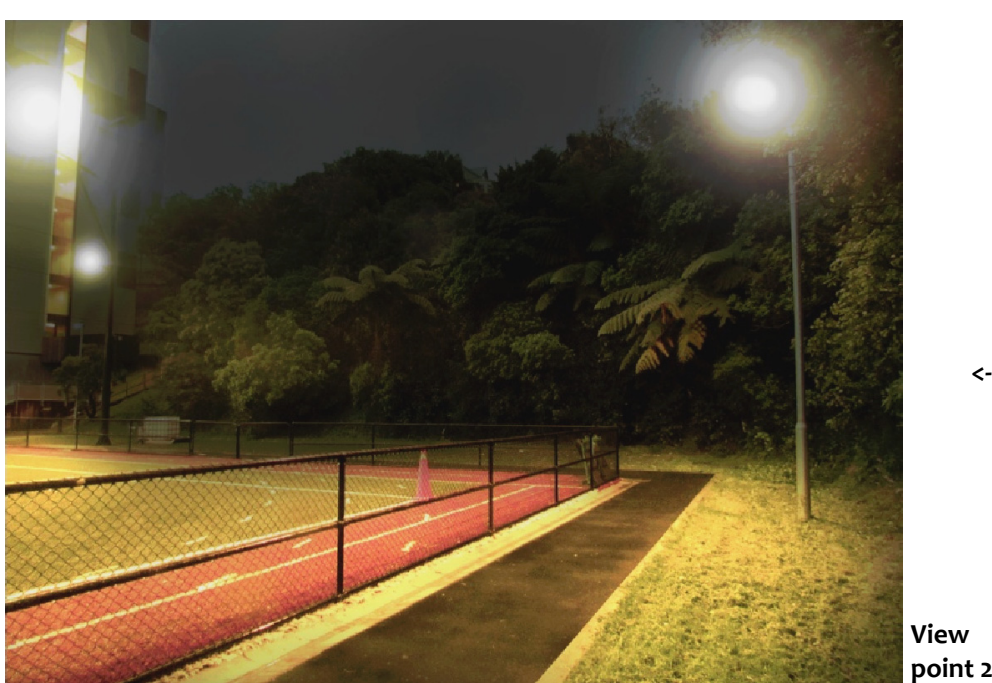

$<$ - Before After ->

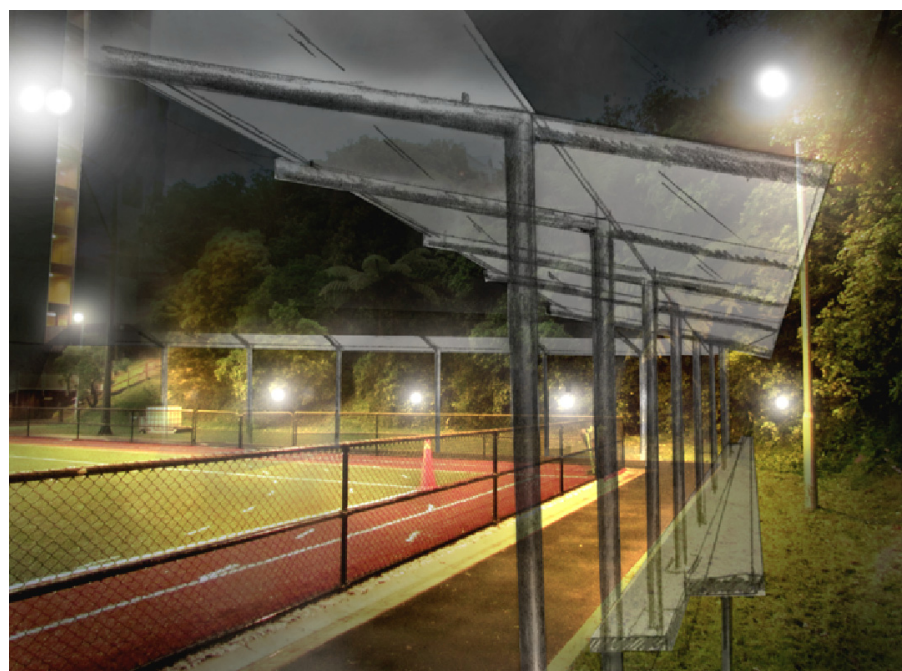




\section{Barrier 'Hot Spot' Zone C: Waiteata Rd}

Figure 71 shows the cognitive surveys highlighted one minor accessibility barrier in 'Barrier Zone C', this was:

- $\quad C P T E D=$ Lack of lighting causing dark areas off the footpath (see for further detail including the design response).

Despite only having a minor barrier, the site was identified in additional 'top down expert' site analysis as being significant for three reasons.

- Firstly, the only area of place-making potential that was identified in the cognitive surveys, 'Place-making Area 4', is along this route (see foldout leaflet). Therefore if the place-making site were to be developed this would increase the reason to improve accessibility along this route in the long term.

- Secondly, the route is one of only two North South connections running across the university; however this route is important because it provides access between the Salamanca Rd Connection and the University sports fields (see Figure 71).

- The connection links Salamanca Rd (North end) to the university sport fields (Southern end) which are used regularly after hours.

- Thirdly, the Northern end of the site provides an 'alternative less steep' route up to the university from Salamanca Rd (see Figure 71), the relevance of this point will be explained next when 'Barrier 'Hot Spot' Zone D' is examined.

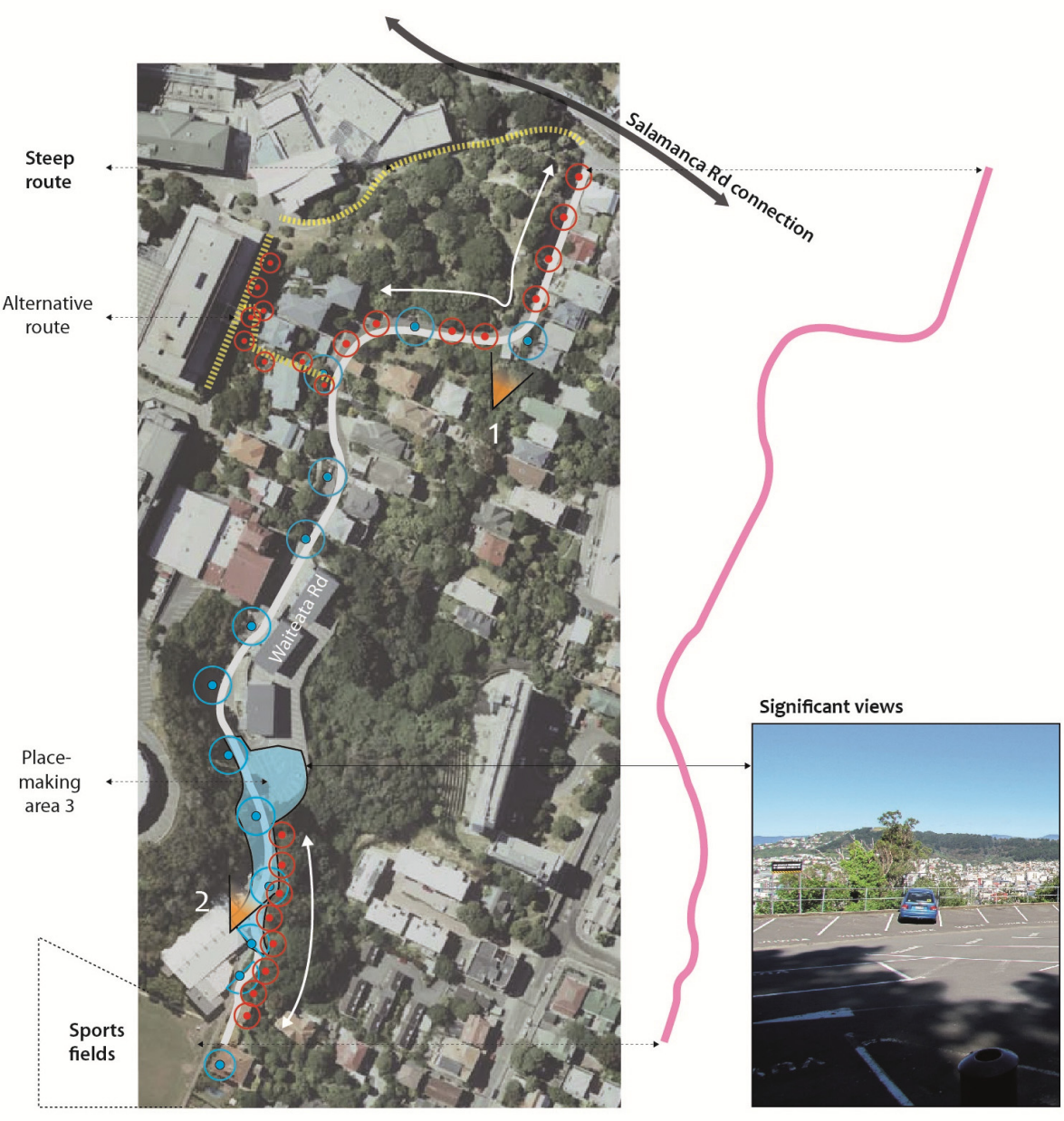

Lighting improvements

$$
\begin{aligned}
& \text { - Existing } \\
& \text { - Proposed additional }
\end{aligned}
$$

Minor barriers

$$
\begin{aligned}
& \text { CPTED Lighting \& Spatial } \\
& \text { - Dark areas off the path }
\end{aligned}
$$

Figure 71: Barrier Zone (C) - minor accessibility barriers and considerations 


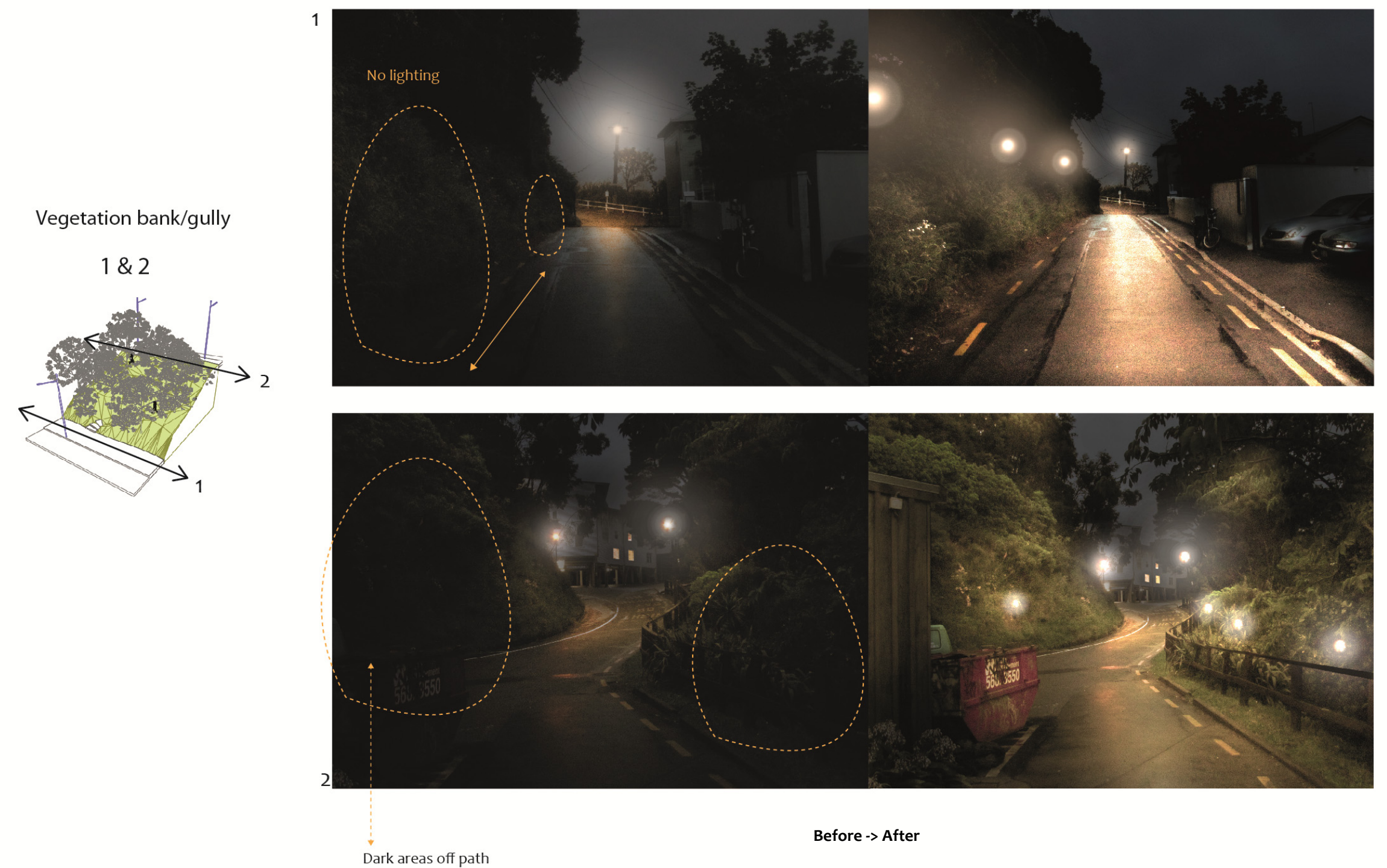




\section{Barrier Zone D and $E$}

Figure 73 shows the cognitive surveys identified two major accessibility barriers in 'Barrier Zone D', these were:

- $\quad$ Path Quality and Ergonomics = Slippery surface and Steep gradient.

- $\quad C P T E D=$ Lack of lighting, and dark hidden areas off the footpath.

As well as two minor accessibility barriers, these were:

- $\quad$ Comfort = uneasiness/creepy presence walking past a graveyard

- $\quad$ Path Quality and Ergonomics = Steep gradient, however this barrier was in another area of site to the other barriers,

Design Response (D)

The CPTED barrier of 'insufficient lighting' could be resolved simply through a lighting strategy using a standardised typological solution which illuminated the vegetation off the path (see Figure 72). However, the solution for the 'steep path gradient' barrier and the 'comfort' barrier, which was caused by views into the nearby graveyard, was more complicated due to the following reasons.

- The duration of the 'steep path gradient' barrier was for 100$150 \mathrm{~m}$, thus excavating a flatter path requires a very high costs.

- $\quad$ The path had no additional opportunities for place-making that could be integrated into any of the solutions to the barriers along this route.
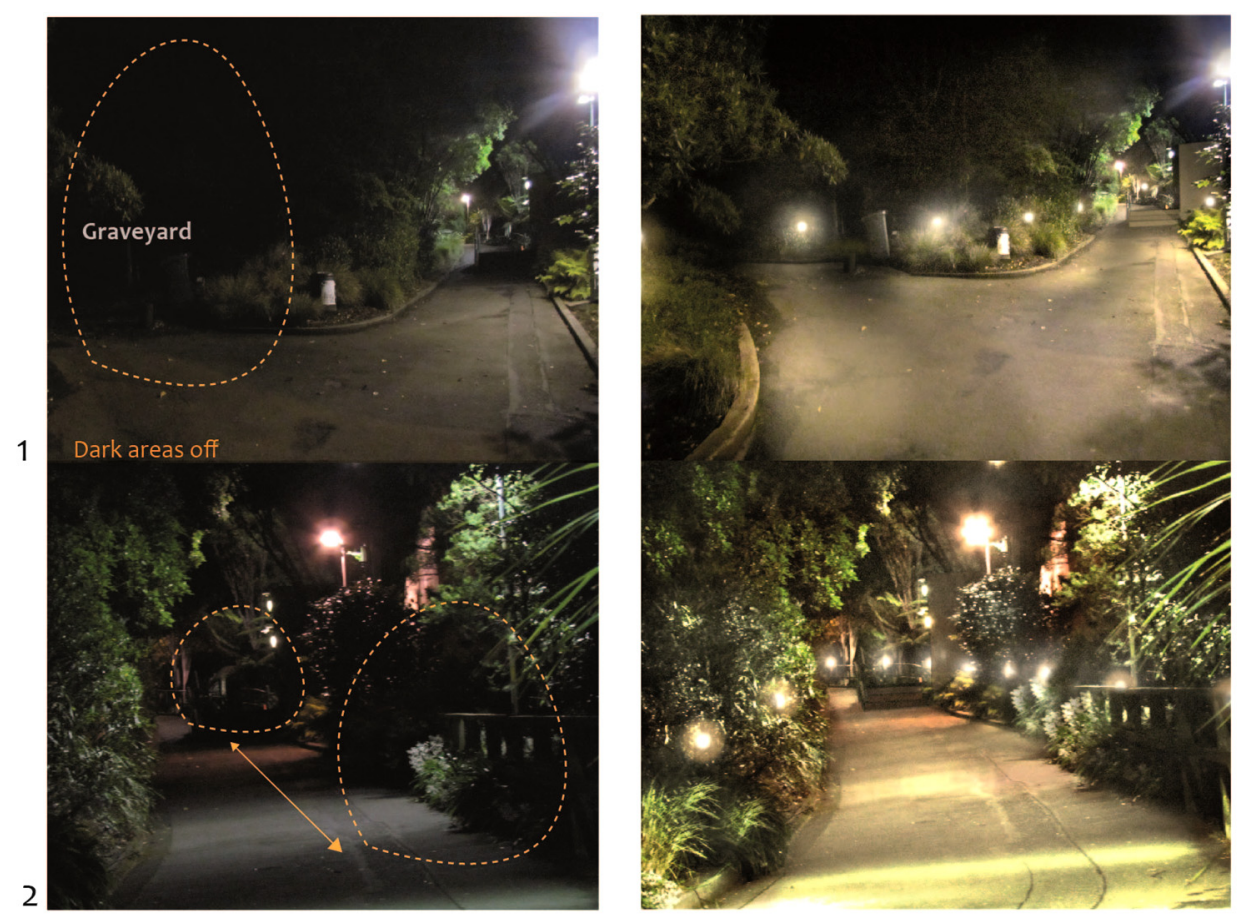

Figure 72: Barrier zone (D) - Lighting strategy Before -> After

Because of these reasons the most effective option to resolving the barriers was to upgrade an alternative route through site. One route in particular through 'place-making area 5', which was inside 'Barrier Zone E' (see figure 73), seemed most appropriate. This was because the route: 


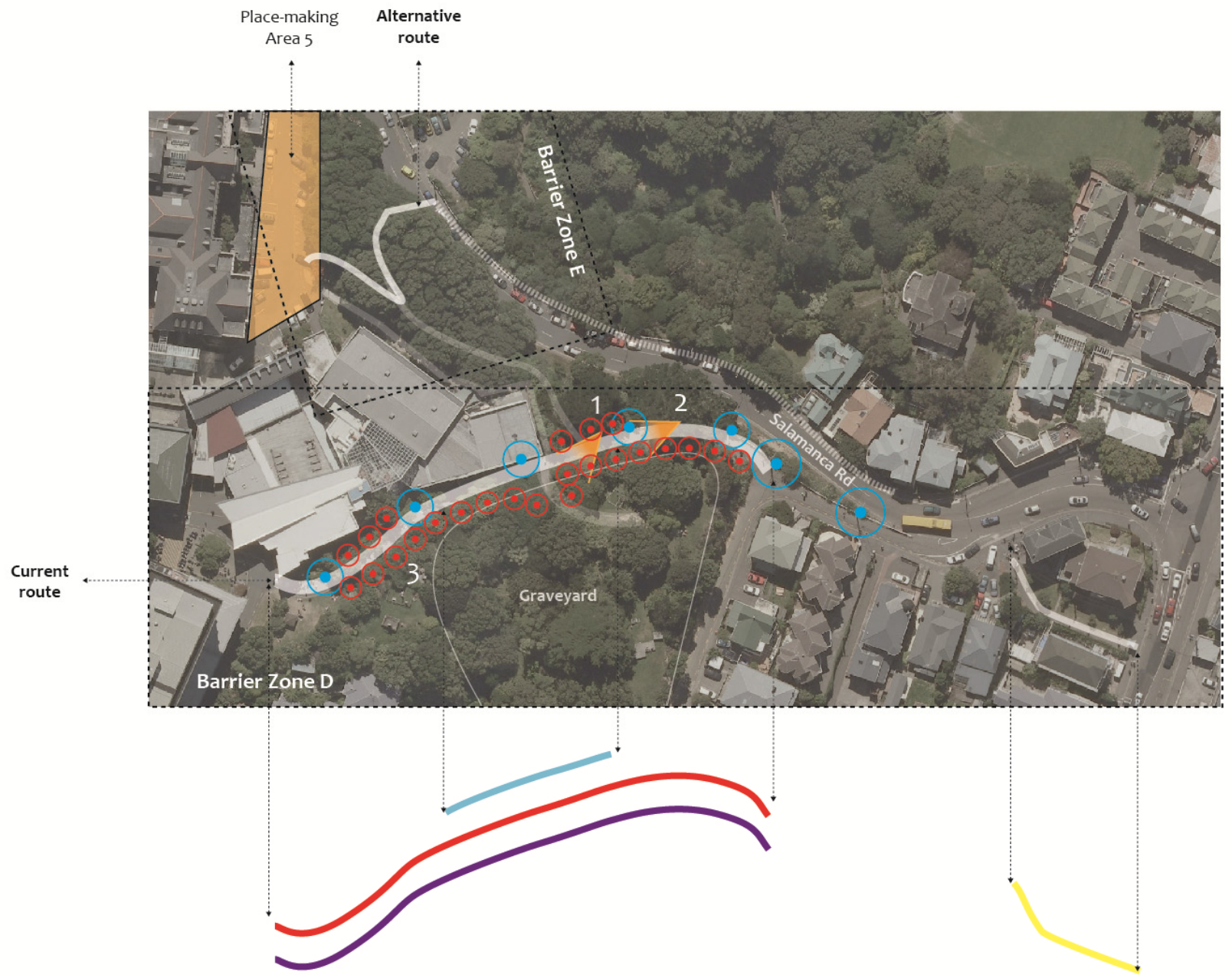

$1,2, \& 3$

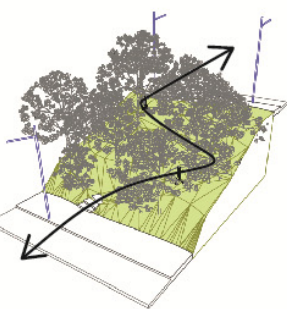

Major Barriers

CPTED Lighting \& Spatial - Absence of lighting

Path quality ergonomics

- Slippery surface

- Steep path gradient

Minor Barriers

Path quality ergonomics

- Steep path gradient

Comfort

- Graveyard

\section{Lighting strategy}

- Existing

- Proposed

Figure 73: Barrier Zone (D) \& (E) accessibility barriers and proposed solution 
Figure 74:

'Thoroughfare bush

typology' CPTED lighting

design guidelines

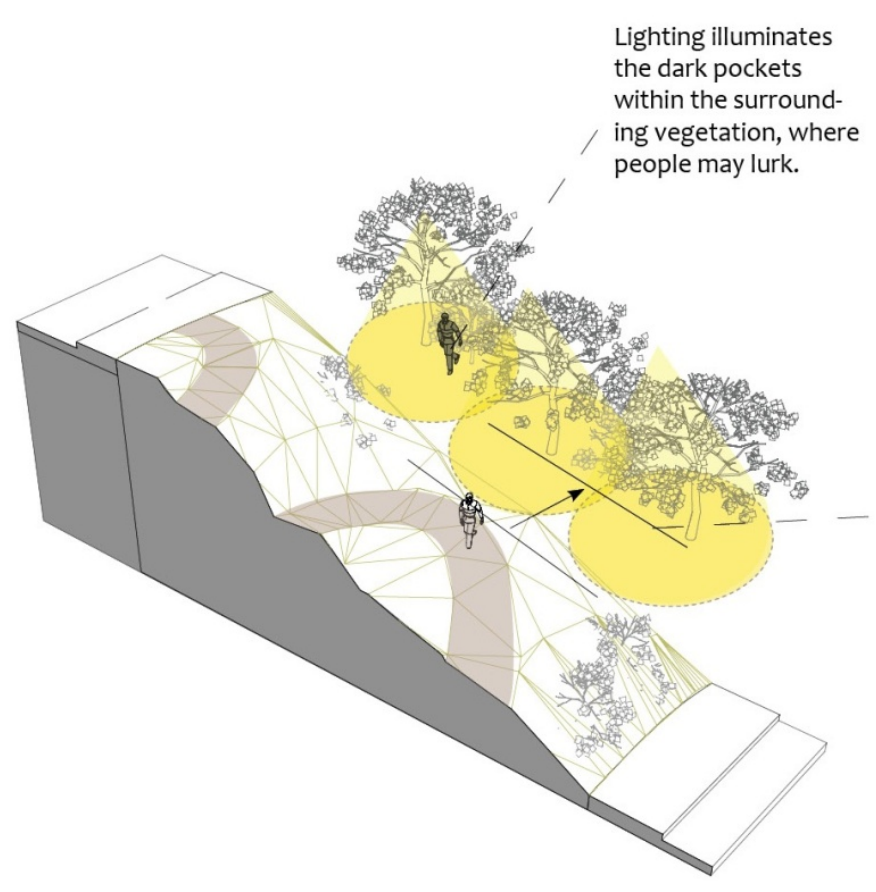

Vegetation cleared back

from path's edge
creating a buffer

zone between

the path user

and the dense

vegetation.
Poor evening path lighting creates strong contrasts, which reduces visibility in shadow. Thus good illumination is achieved through multiple source lighting.

1
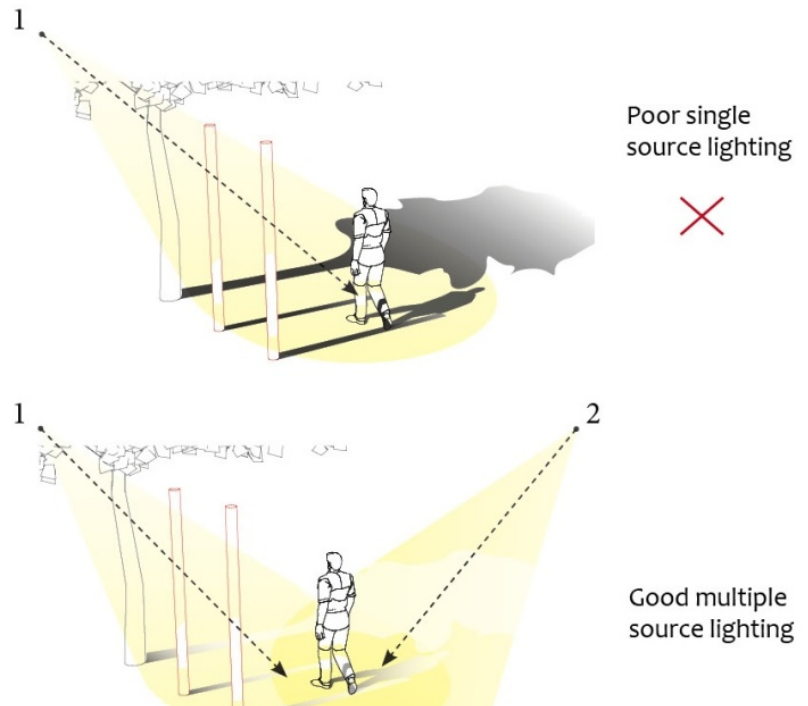

- $\quad$ Bypassed the graveyard; and was over a flatter gradient than the

current route;

- The route passed through place-making 'Area 5', which was identified as having significant place-making potential (Figure $36 \mathrm{p103).}$

- Was on a key edge mediating access between to an iconic University

Hunter building, and a major commuting corridor (Salamanca Rd)

between 'Residential Catchment A' and the CBD.
Note the solution for this alternative route of proving safe access between Salamanca and University was designed with guidance from the 'thoroughfare bush' typological study (Figure $74 \& 75$ ). 
Figure 75: 'Thoroughfare bush typology' CPTED

spatial design guidelines

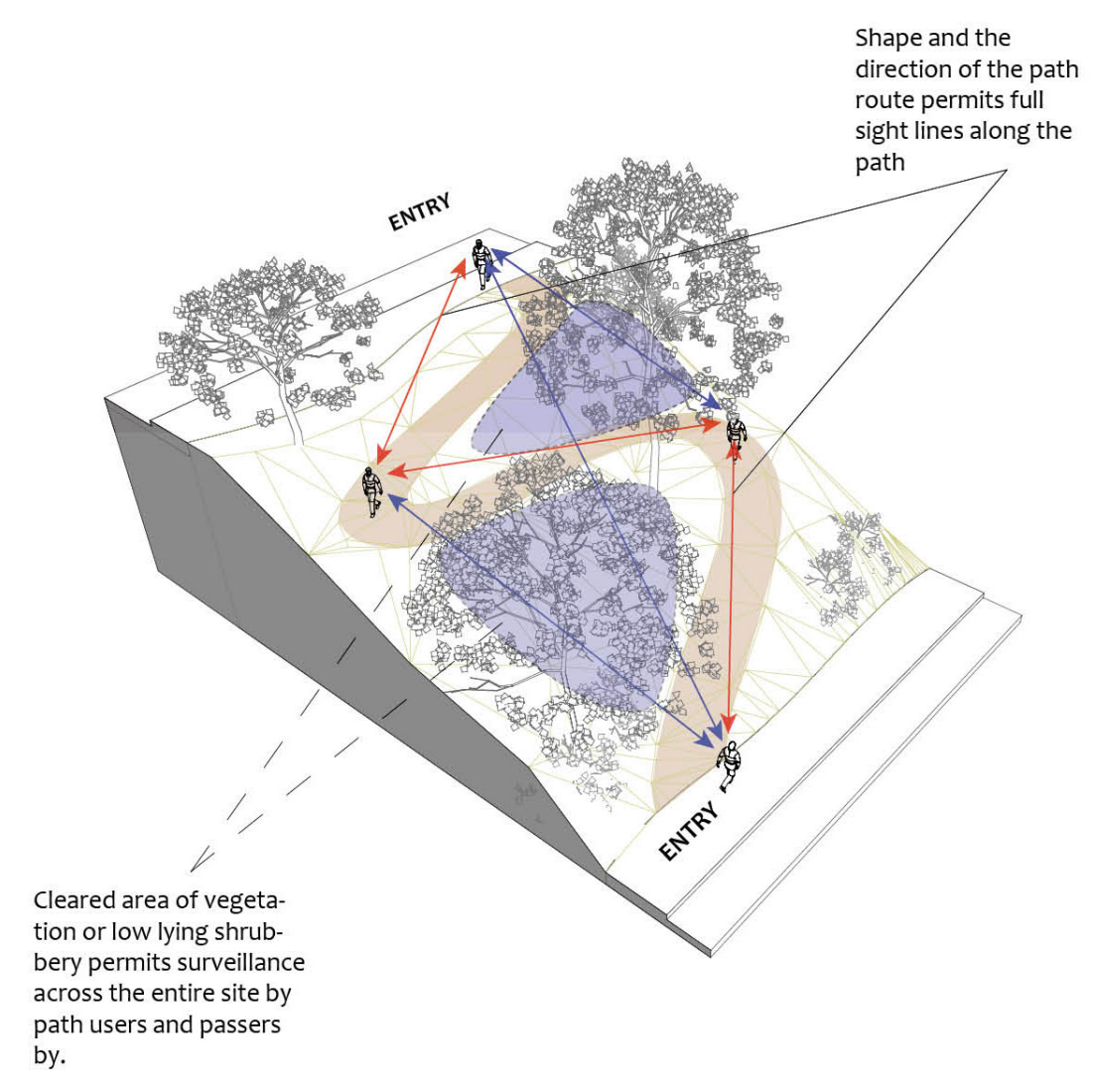

Trees pruned

to above head

height to permit

visibility.

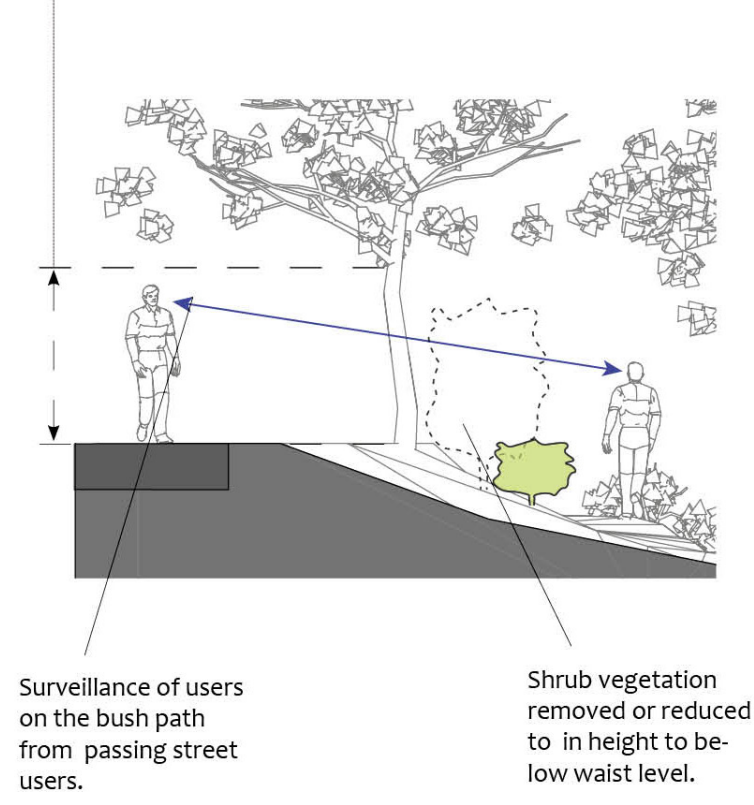




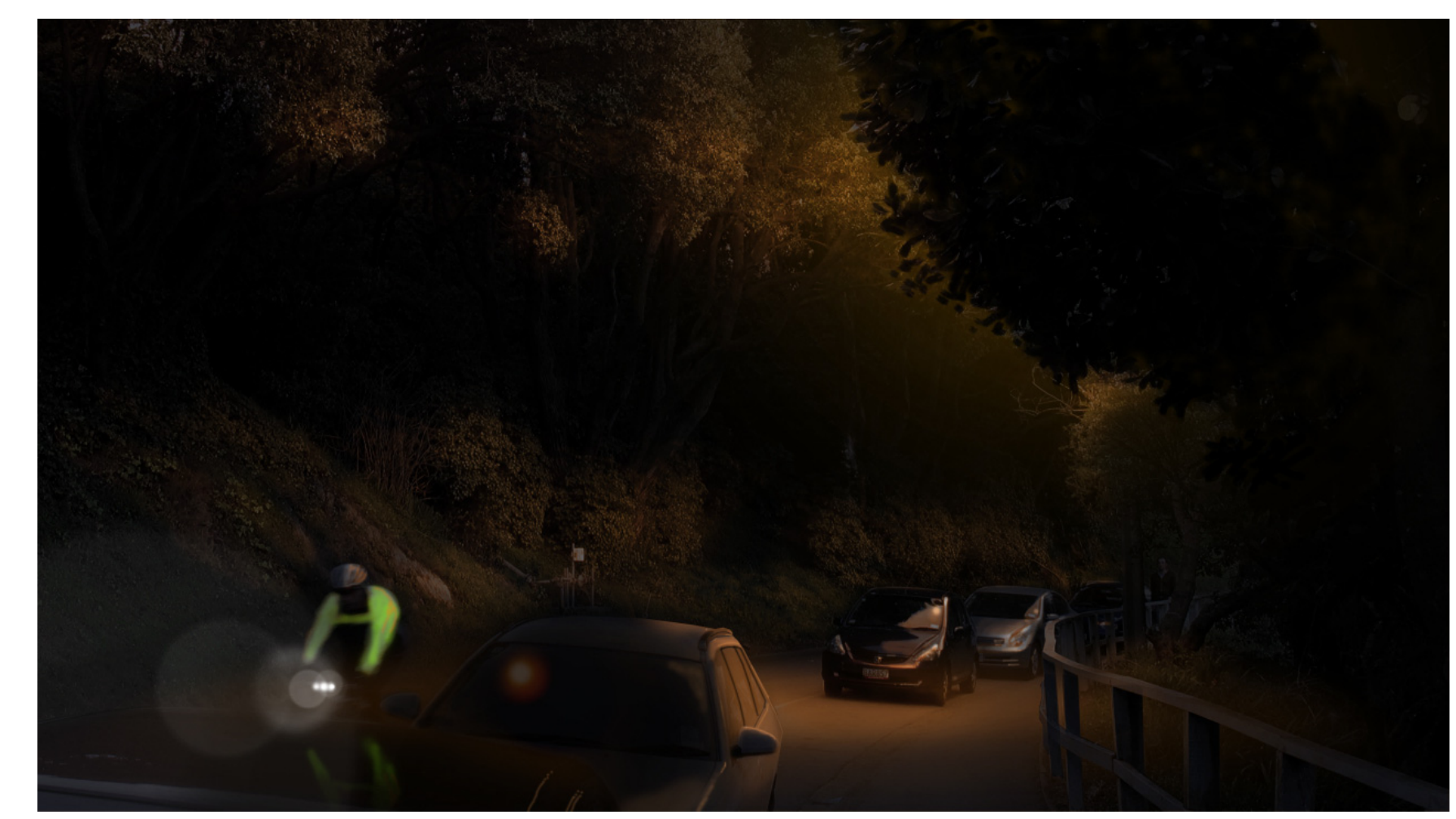

Figure 76: Barrier Zone (E) - Existing hidden path up to the University - Before -> After 


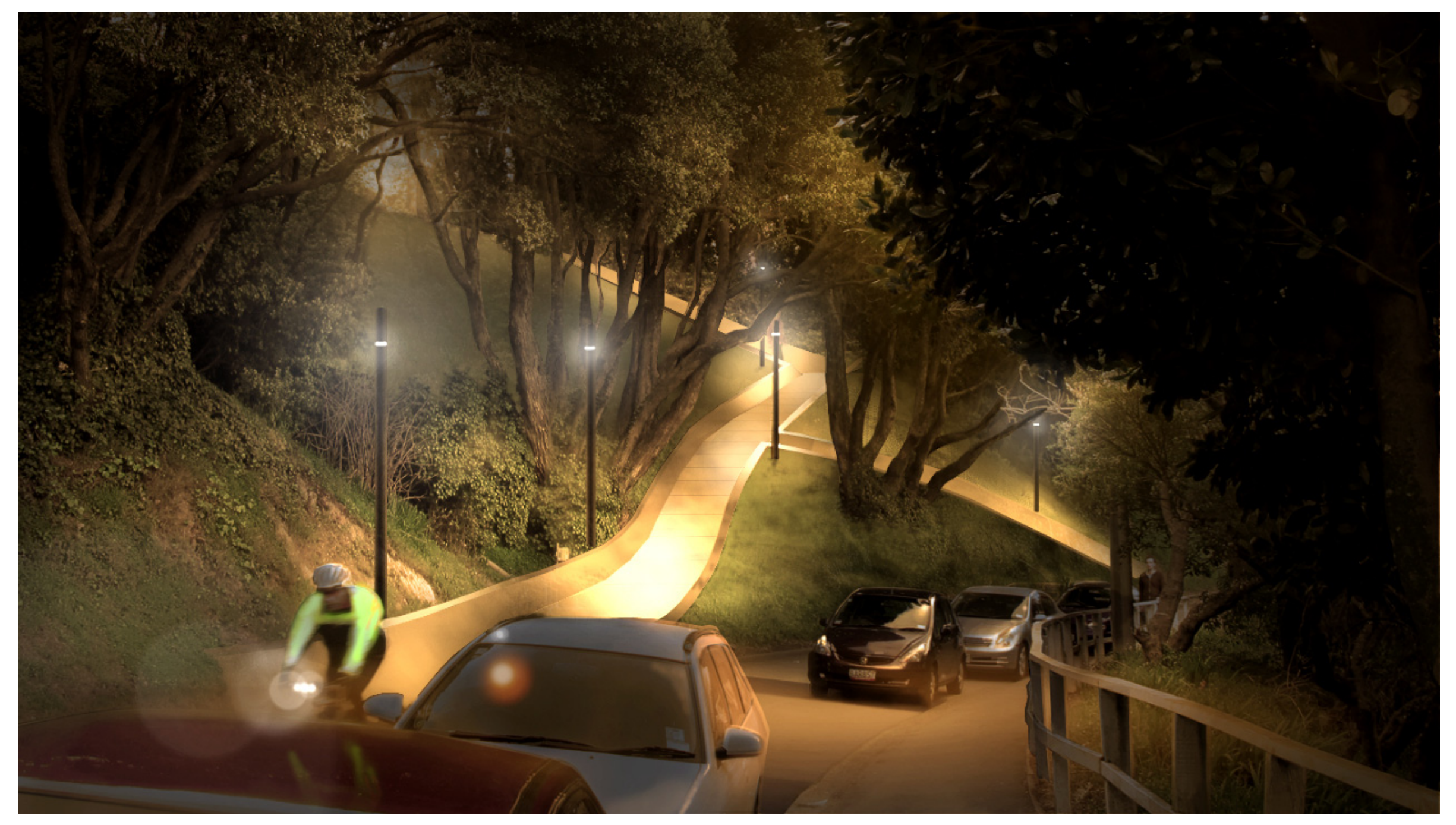




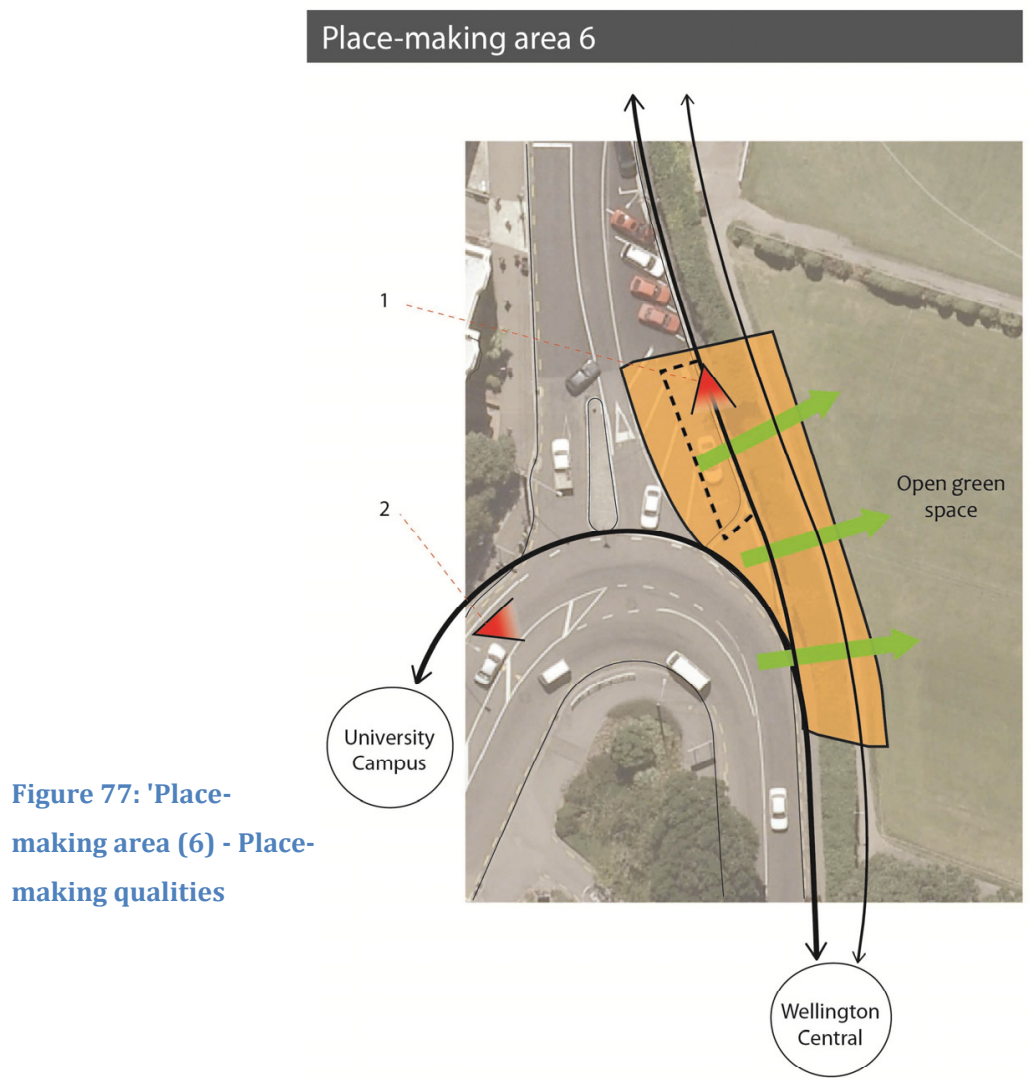

\section{Design Response (E)}

The one minor barrier identified in 'Barrier Zone E' was the 'lack of shelter' present along Salamanca Rd (Figure 48 p126). The area was also identified as having place-making potential due to the potential for views

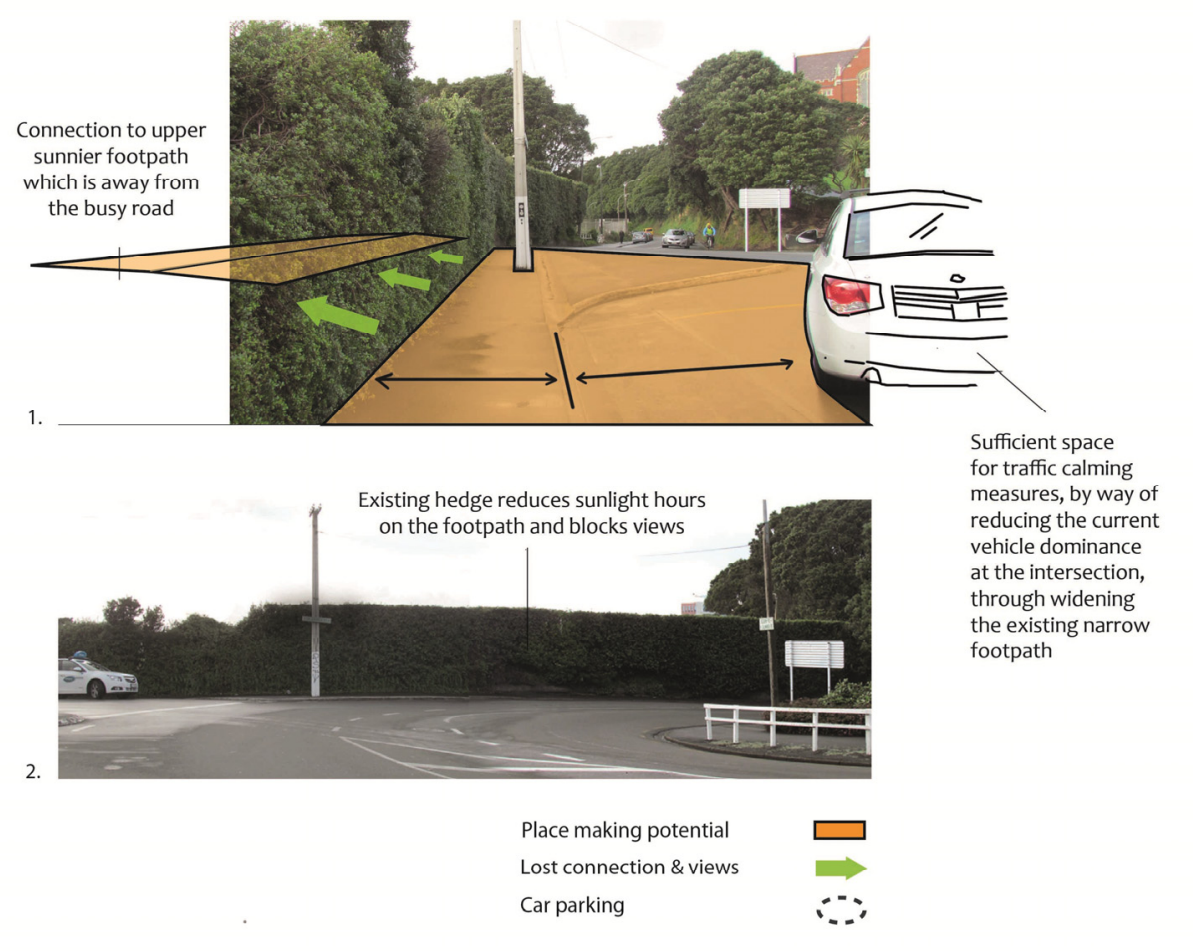

across a large green space towards the city beyond, whilst also reconnecting the main footpath with an upper adjacent footpath Figure 77. The design response was produced previously in Chapter 6.3 'Design Experiment 3' (refer to Figure 39 p111). 


\section{Barrier Zone F}

Site Analysis

In the last barrier zone, 'Zone ' $F$ ' (see back fold out leaflet), one minor accessibility barrier was identified, this was:

- $\quad$ Comfort $=$ No shelter from weather (Figure 79).

The relevant spatial conditions of each barrier typology that need to be considered in the design were:

- Urban residential

- The design intervention should be considerate to the visual character of the houses. For 'Barrier Zone F' this was a row of Victorian buildings on the Western footpath (Figure 79).

\section{- Vegetation}

- The built landscaping features, and dense vegetation can create hidden or secluded areas.

Design Response

In response to these points the concept (Figure 80) was designed. The design resolves the barrier of 'no shelter from weather' by providing a mixture of vegetation canopies and built canopies which create one main covered route along Kelburn rise. The design is successful in a two ways:
1. By having the canopy running along the Eastern footpath of Kelburn rise Rd, the aesthetic presence of the character buildings along the Western footpath is retained.

2. The design is cost effective because the proposed canopy requires little intervention:

- $\quad$ The existing trees along the Eastern footpath already provide the majority of cover, thus only some additional canopy tree planting is needed.

- Where it is not feasible to plant a vegetation canopy, i.e. because of limited footpath space, the proposed design resolves the lack of shelter efficiently by using a standardised typological canopy solution (Figure 81), this solution was developed previously in 'Design Experiment 2' (Figure 27 p89).

As one final comment, although the vegetation canopy along the Eastern footpath would take a long time to mature, and even then would not grantee complete shelter from weather; this is justified considering:

- The barrier on site was only a minor barrier with no placemaking potential;

- $\quad$ The barrier was considered as 'Stage 3' in the hierarchical framework (Figure $60 \mathrm{p137}$ ) and therefore it is only of medium urgency to implement. 


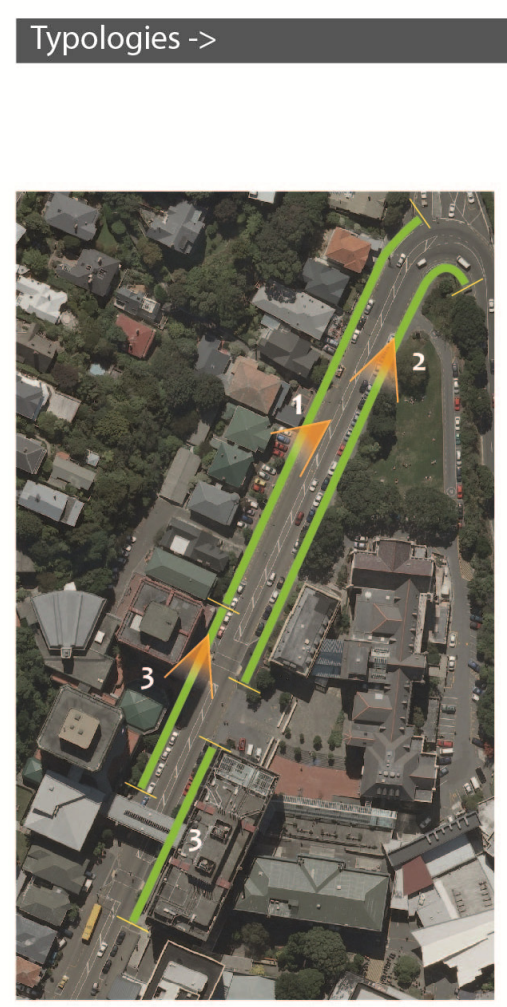

Comfort

No shelter from weather

Urban residential

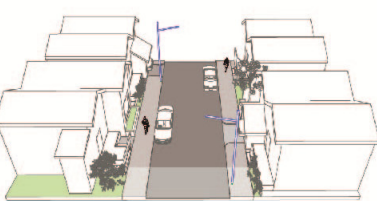

Comfort

- No shelter from weather

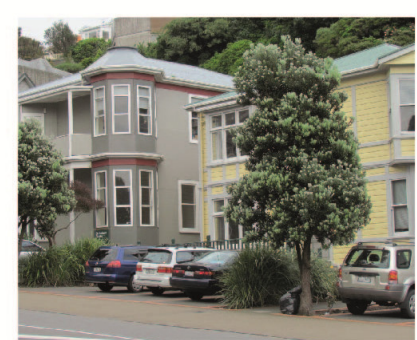

Cars tuning into off st car parking logistically make it difficult to

build canopies along the

footpath.
Vegetation

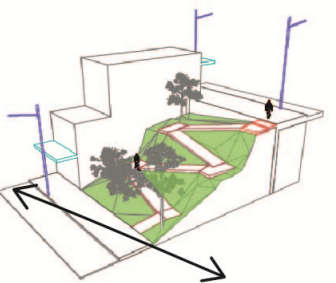

Comfort

- No shelter from weather

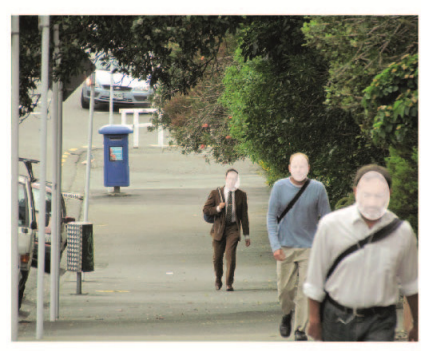

Partially developed tree canopy provides some shelter.
Commercial back st

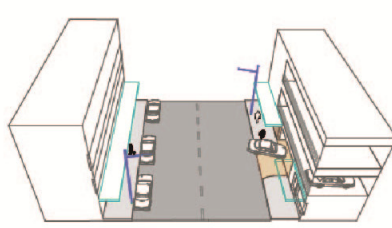

Comfort

No shelter from weather

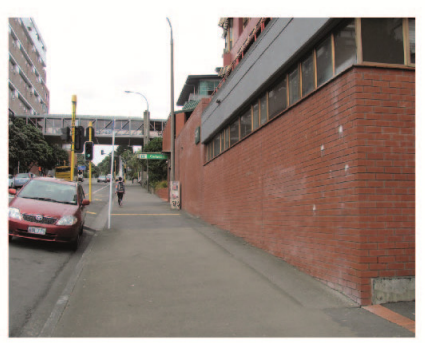

Canopies can easily be built in front of blank walls or retrofitted to them.

Figure 79: Barrier Zone (F) - accessibility barriers and additional considerations 


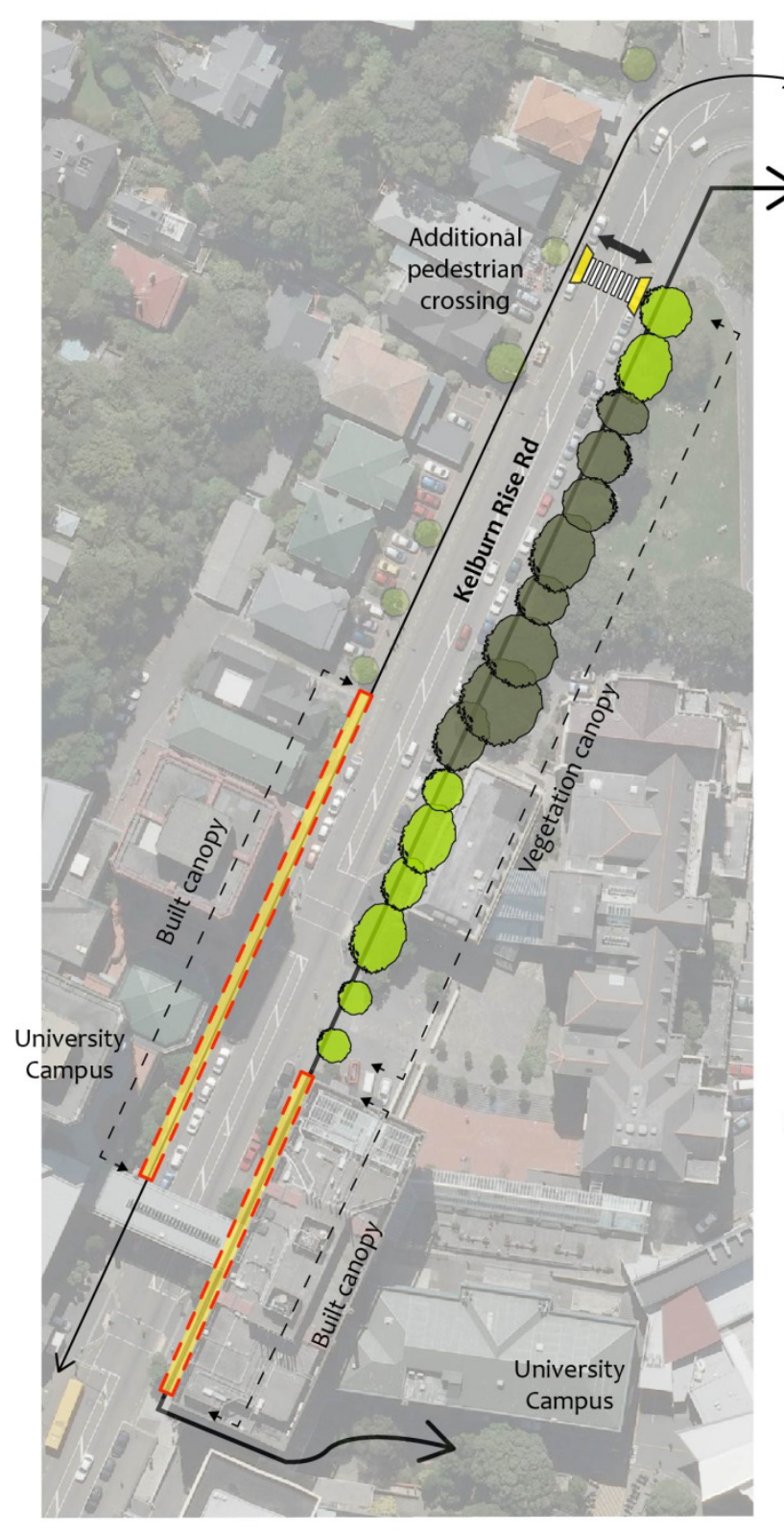

Primary route

during calm

weather

Primary route

during extreme

weather

Key

Proposed built canopy Proposed new trees

Existing trees

Figure 80: Barrier zone (F) - Proposed shelter intervention

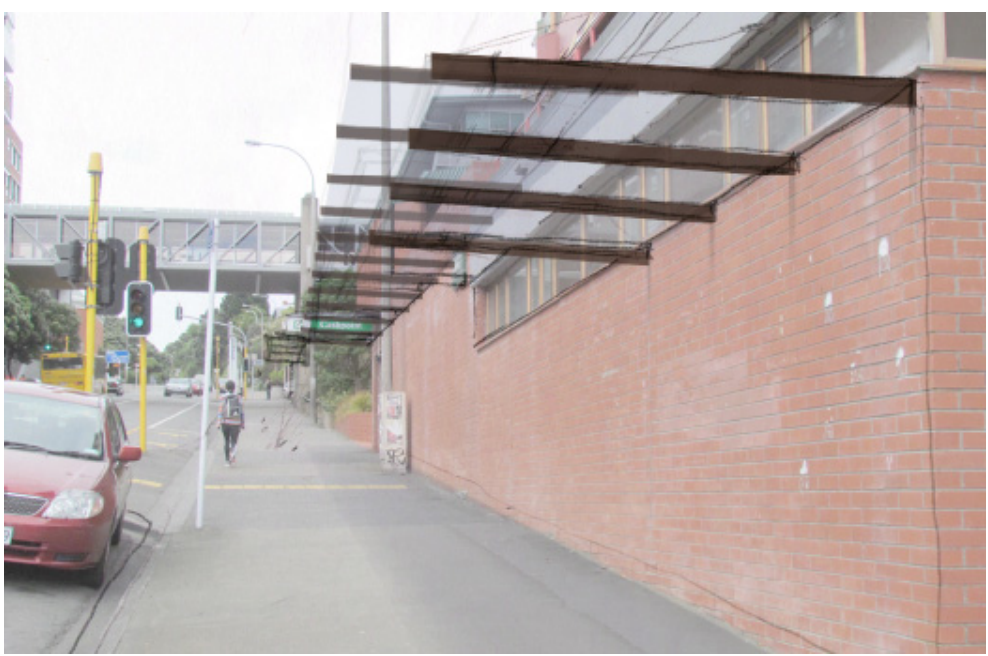

Figure 81: View point ' 3 ' artist impression of standardised typological canopy

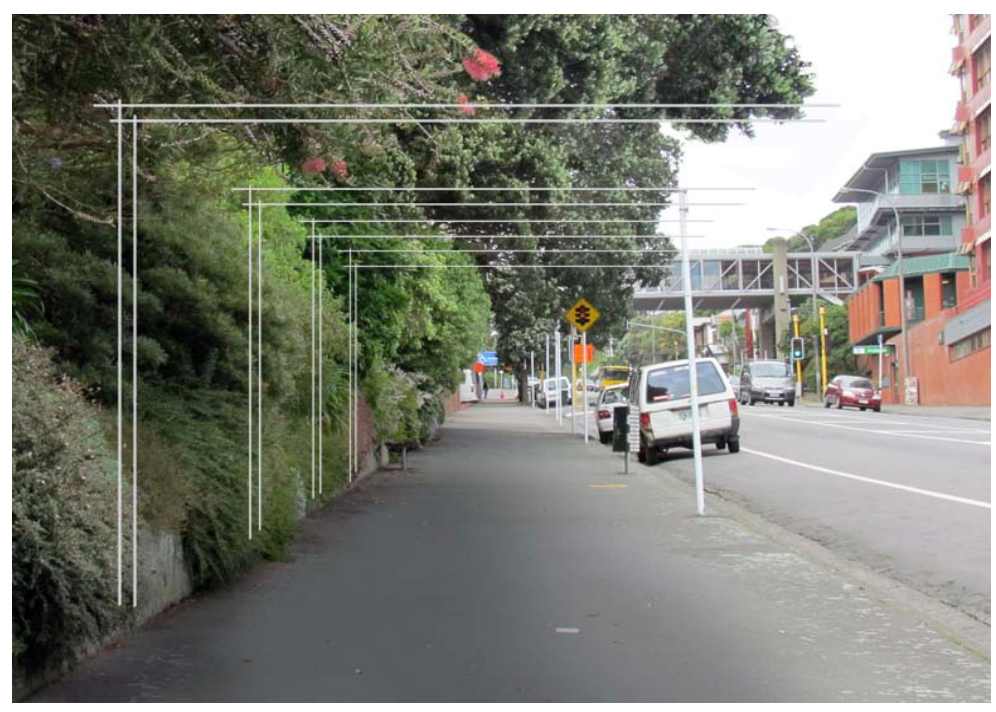

Figure 82: View point '2' artist impression of vegetation canopy 


\subsubsection{Results}

A number of observations can be made about the 'combined methodology' used in Design Experiment 4, in particular how successful it was as a method for researching, designing, and implementing pedestrian infrastructure. These observations will be discussed in the following sections:

1. The Cognitive Survey Process

- Developments made in the cognitive survey method of analysis.

- The limitations of using the cognitive survey as a standalone method of site analysis.

2. The Emerging Design Themes in the 'New Pedestrian Design Strategy'

- The tension between design standardisation and design customisation.

- The role of the 'expert' in the pedestrian design process.

\section{The Cognitive Survey Process}

Developments Made in the Cognitive Survey Method of Analysis

Having completed two separate cognitive surveys in this thesis, the first in 'Design Experiment 2, and the second in this experiment, three observations can be made about how well they performed as a method of identifying accessibility barriers on site.

1

Firstly, the survey in 'Design Experiment 4' was more efficient than the survey used in 'Design Experiment 2' despite interviewing a larger sample size. This was because the experiment found that multiple surveys could in fact be conducted, in groups of up to four people at a time, whilst still maintaining quality in the respondents' answers. The advantage of this was more surveys could be carried out, which subsequently led to a more cost efficient survey process.

Secondly, compared to the cognitive survey in 'Design Experiment 2', in Design Experiment 4's survey there was an increase in the level of detail respondents were recalling about the site. This resulted in the respondents identifying more precisely the location of and areas of placemaking potential on the survey map. I argue that this improvement in detail was because 'Design Experiment 4's survey focused on a much smaller area than the survey in 'Design Experiment 2', which survey boundary covered the whole Central Wellington area. The advantage of 'Design Experiment 4's' survey was that it focused on a smaller area of the city but in greater detail; and also that a larger survey map was used for the respondents draw on. This meant more of the respondent's interview 
time was spent examining the site. And as a result the detail of the respondents' answers improved

This notable improvement in detail was significant for two reasons regarding researching accessibility barriers on site:

- Firstly, when the surveys were collated it led to a more accurate map of where and what the accessibility barriers were on site.

- Secondly, it confirmed after that more information could be learnt about the accessibility barriers in a specific area of site when a second cognitive survey is carried out for each 'barrier hot spot' that was identified in the first survey.

3

Thirdly, the larger survey sample size in 'Design Experiment 4' caused new accessibility barriers to emerge that had not been raised in the accessibility literature. An example of this was the unsettling presence that the respondents raised numerous times from walking past the graveyard in 'Barrier Zone D'. As a result a new kind of accessibility sub category was identified, I have called this the 'psychological atmosphere' of a place. This finding is significant as it demonstrates that the accessibility barriers people react to in the built environment are unique to every site, and therefore why the public needs to be consulted with in order to understand what pedestrian accessibility barriers affect their travel behaviour.

\section{Limitations of The Cognitive Survey (deciphering the data)}

In 'Design Experiment 4' the cognitive survey data was to identify the areas of moderate and major accessibility barriers, as well as areas of place-making potential in a site. In this process the level each area was graded depended on the number of comments it received by the respondents in the surveys. This, however, caused a problem as it limited the extent accessibility was understood on site.

For example, when zero respondents walk through an area of site, thus causing no accessibility barriers to be raised, the cognitive survey analysis presents an illusion that there is no accessibility barriers present in the area. This caused the problem of how to identify pedestrian barriers in areas of site where few respondents have been; and also, how to predict what barriers would be present when new pedestrian routes were developed through unused sites.

Note these same problems also apply when researching the place-making potential in a site. For example in 'Design Experiment 4' the cognitive survey results suggested that there were very few areas of site that were considered to have place-making potential. However, arguably this could have been because the survey respondents were yet to experience all areas of site.

These finding are significant because they highlight that it is important to accompany a 'bottom up' cognitive survey with a 'top down expert' 
method of site analysis i.e. the typological study of pedestrian accessibility, when researching accessibility barriers and place-making within a site. This is because an additional method of site analysis will give design professionals an insight into what kinds of barriers pedestrians are likely to encounter in unfamiliar sites, and therefore what barriers need to be addressed in their design intervention.

\section{Two Emerging Design Themes in the 'New}

\section{Pedestrian Design Strategy'}

(Theme 1): Design standardisation versus Design customisation

The first emerging theme in this research is around the question,

\section{$?$}

What is the role of the design 'expert' in a pedestrian design process which aims to achieve two contrasting ideologies - cost efficiency through design standardisation; and responsiveness to context through design customisation?
One of the successes of the 'New Pedestrian Design Strategy is that it combined two design approaches, from the previous design experiments 2 and 3 , into a new approach for designing pedestrian infrastructure. These approaches were:

- Standardised typological approach

- Customised place-making approach

In reflection of 'Design Experiment 4', this thesis argues role of the 'expert' is to ensure there is a balance between design standardisation, and customisation when implementing pedestrian infrastructure. However, this balance creates a tension between a standardised design, which is economically efficient, versus a customised design that improves the quality of the surrounding environment by being responsive to context.

This thesis acknowledges that design customisation has obvious benefits over standardisation in terms of how it responds contextually to the surrounding environment; for example, there are areas of place-making potential hidden within site that can only be realised through a customised design process which allows them to be identified. However on the other hand, little acknowledgement has been made towards the benefits of design standardisation. These benefits are:

- Commonality and therefore improved legibility in terms of pedestrian way finding, 
- Cost efficiency through mass production, modularity of the design, and one off design authorship, thus reducing the amount of resources required for the design. This can be seen in the Wellington example of the Adshel bus shelter (Figure 23 pg 83).

Furthermore, because of these low cost benefits, one could even argue the standardisation process is good for improving overall pedestrian accessibility in a site as it enables a wider scope of projects to be completed.

However, despite the cost benefits, the consequence of standardisation is a reduction in the quality of the urban environment, as standardised typological solutions lack response to context. This is significant as it has implications on the role of how pedestrian access and land use typologies are used in the pedestrian design process. For example, until now the purpose of the typological study was to be a library of information explaining the spatial principles of how the design of the built environment affects pedestrian accessibility. In this sense the typologies have provided a useful way of simplifying the complex real world environment through a theoretical lens, which was pedestrian accessibility. 'Design Experiment 4' exposed that standardised typological solutions as a design approach can still be used effectively if done in the right manner. However, they require an additional stage of development. Each typology in the study needs to have a diagrammatic explanation of the spatial principles that have to be considered in order to maintain a high level of accessibility. Figure 75 p157 is an example of how the 'Bush thoroughfare typology' could be developed further and become more useful. This improvement to the typology library is significant because it can help the design customisation process become more efficient in two ways:

- Firstly, it would make it easier for design professionals to include designing for pedestrian accessibility in their interventions;

- Secondly, it would give pedestrian design practice in Central Wellington a theoretical spatial guideline of how pedestrian accessibility is affected by each typology in the built environment.

\section{(Theme 2): The Role of the Expert Designer}

In addition to this, 'Design Experiment 4' has shown that within a longterm strategy, the role of the design 'expert' also varies between micro and macro scale levels of intervention. At a micro scale the 'experts' role is to ensure a design is responsive to its immediate context. This means the designer must be:

- Aware of areas within a site that have place-making potential;

- Conscious of contextual qualities like materiality and form;

- Responsive to the activities that occur in the surrounding environment outside of the foot path. 
In contrast to this, at a macro scale the expert has the ability to influence pedestrian accessibility within the wider pedestrian network. Thus at the macro scale the designer's role is instead to understand:

- How the greater accessibility and place-making systems function within the wider urban context;

- What are the major and minor pedestrian barriers are affecting accessibility in the wider urban context.

- $\quad$ And therefore, what barriers of the site are of greater urgency to resolve than others.

As a final point this thesis argues that the role of the 'expert' designer is also to be conscious of the design standardisation versus design customisation playoff, in that both solutions should be used together under one strategy for designing pedestrian infrastructure at a micro and macro scale. This is because if the designer is familiar with the minor and major accessibility barriers within site, as well as the areas of placemaking potential, the designer can identify within site where it is most appropriate to use standardised typological solutions, or customised sitespecific solutions. Therefore by understanding all these attributes of site, the designer can plan how to address pedestrian accessibility and place making through one effective strategy at both the micro and macro scales.

\subsubsection{Further Direction}

The previous findings required the 'New Pedestrian Design Strategy' to be altered again: 
4.

Conduct analysis of the greater urban

context in order to see which accessibility

barriers and place-making opportunities,

which were identified through the cognitive

surveys, are of most importance to improving

the larger pedestrian and public space net-

work.

What are long-term changes that need to be made within the larger urban context in order to improve: connectivity of the pedestrian network, as well as the quality of public space network, at a macro scale? survey specific to the wider 'hot spot' area in order of highlight the barriers and

perceived place-making qualities of all the pedestrian routes throughout the site. This is important because other routes may present a more affordable alternative to resolving the 'barrier hot spot', or may present better opportunities for

place-making.

2. Conduct a citywide survey using the cognitive interview method, to highlight the 'hot spots' where accessibility barriers are present in the city's pedestrian network. In particular between the residential catchments and the CBD.

‘Bottom up’ approach

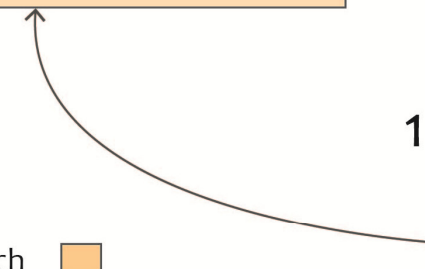

'Top down' approach

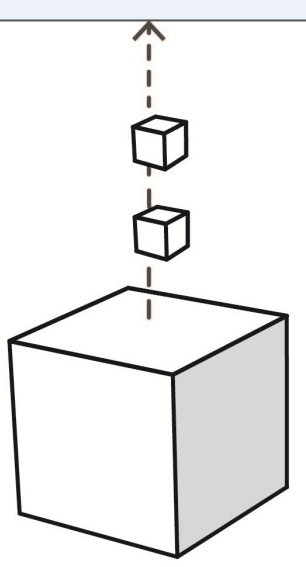

- Access typologies

- Land use typologies

1. Carry out a Typological study of pedestrian accessibility for Central Wellington as a baseline for understanding in theory how accessibility is affected by the design of the built environment.
Library of pedestrian:
5.

Within the key areas identified in the previous macro urban analysis, particularly where the cognitive survey data was

insufficient, conduct a further 'top down' expert analysis at the micro scale. In order to uncover any additional accessibility barriers and areas of place-making potential.

6.

Locate the access typologies on site in order to understand the spatial principles that are causing each significant barrier. Therefore becoming familiar with the level of resources required to resolve the barrier.

7. Use a cost versus barrier urgency framework to design a hierarchica strategy that organises into various stages how the place-making and accessibility barrier solutions will be implemented within the wider hotspot area 
The nature of this thesis is multidisciplinary, drawing theory from areas of behavioural psychology, geography, policy, urban design and architecture. The contribution of this thesis, therefore, is relevant across a range of disciplines. In particular this thesis has demonstrated how these disciplines, together, can improve the way in which pedestrian infrastructure is researched, understood, and designed by authorities and professionals. This chapter will discuss the contributions of this thesis to contemporary practice. The chapter will be discussed in two parts:

1. Why using a combined 'Top down' and 'Bottom up' approach to researching, designing, and implementing pedestrian infrastructure can improve pedestrian design practice in Central Wellington.

The 'New Pedestrian Design Strategy' and Contributions to Contemporary Theory and Practice

2. How typologies can be applied to the professions of architecture and urban design.

\section{(Part 1) 6.1 Improving Pedestrian Design Practice in}

\section{Central Wellington:}

The purpose of the initial 'Hypothesis Pedestrian Design Strategy' was to develop an understanding of how people perceive pedestrian accessibility in the built environment. In particular, how people's walking behaviour could be influenced intentionally through the design of the built 
environment. This thesis adopted a four-paradigm framework, which was developed by Taylor, Zube and Sell in order to remove unwanted variability in perception research. This framework was applied to the initial 'Hypothesis Pedestrian Design Strategy' as a way of ensuring a thorough investigation of Wellington's travel behavioural walking problem. Over four design experiments, the initial 'Hypothesis Pedestrian Design Strategy' was developed further into a 'New Pedestrian Design Strategy'. In the final design experiment, 'Experiment 4', the 'New Pedestrian Design Strategy' demonstrated that using a combined 'top down expert' and 'bottom up qualitative' approach, at both a micro and macro scale, could improve the cost efficiency and quality of the design outcome, when researching, designing, and implementing pedestrian infrastructure.

Using the findings from the design experiments, the following argument will explain how the current processes Wellington local authorities use to implement pedestrian infrastructure could be improved if a multi paradigm approach was adopted. The argument will start by re-capping the three main issues with Wellington's current process, and then close by explaining how this process could be improved by adopting the new strategy developed in this thesis.
The Problems with Wellington's Current Pedestrian

\section{Design Process:}

Problem 1: The Techniques Used to Identify Pedestrian Accessibility Barriers

The first problem is regarding the techniques used to research pedestrian accessibility barriers in Central Wellington. Currently there are two kinds of processes used by Wellington authorities for this, and both are subject to criticism. These processes are:

- $\quad$ The 'top down' expert master-plan - The City to Sea Waterfront study by Jan Gehl,

- The 'bottom up' NZTA Community Street Audit,

\section{'Top down' Expert Master-Plan}

Jan Gehl's 'top down' approach is problematic because it fails to identify three key issues:

- Firstly, because Gehl's approach is not qualitative, and instead uses expert analysis to make educated judgments of where pedestrian barriers are in the city, his approach fails to identify:

- What pedestrians' perceive to be the most significant barriers to walking in the built environment,

- Where these barriers are located in the built environment 
- The specific spatial conditions that have led the barriers to occur in the first place.

Gehl's designs lack focus in addressing the pedestrian accessibility barriers that are of greatest public concern. This causes Gehl's design interventions to be less cost efficient and effective.

- Secondly, Gehl's approach is beneficial in providing an overall framework of direction, by offering general principles towards improving the quality of the pedestrian environment. These guidelines and principles, however, lack detail and specificity to Central Wellington, and thus do not provide a complete solution to Wellington's accessibility problems.

- Thirdly, Gehl's approach is limited in its scope as it only addresses the CBD area. As a result it leaves the highly important areas between the residential catchments surrounding the CBD, neglected. Note that these areas fall outside the intentions of Gehl's study and this exposes large areas within Wellington's planning initiatives that need to be attended to, in order to improve accessibility across the wider pedestrian network.

\section{The 'bottom up' NZTA Community Street Audit}

The second approach is the 'bottom up' and qualitative 'Community Street Audit', which is used by the NZTA to identify barriers to walking in the built environment. This approach is problematic for two reasons:

- Firstly, the 'on site walkthrough' method requires significant organisation of the survey participants, is time consuming, resource heavy, and limited to small-scale analysis. This is a significant problem as the method is therefore inappropriate for identifying barriers on a large city-wide area as the survey group and site area would be too large.

- Secondly, the survey technique used to question the respondents is flawed in the following ways:

1. The survey questions are prescriptive in that they force participants to answer a range of prescribed questions. As a result, participants are not given the opportunity to highlight the barriers they consider most significant, therefore causing the technique to be less accurate.

2. The 'on site walk-through' technique is also problematic as it puts emphasis on respondents' using observation to identify the barriers they perceived in the surrounding environment. This makes it difficult to prevent respondents from over searching the surroundings, and identifying carelessly any objects/spaces as accessibility barriers, just because they were present at the time of the interviews. This also reduces the level respondents will refer to their mental representation of the environment to recall barriers, which is significant 
considering behavioural theory states that people's

travel decisions are reactions to their mental

representation of the environment.

- Thirdly, because the walk through technique operates within the context of the footpath, the technique is limited to only improving accessibility at the micro scale. For example improving the quality of the footpath surface, and road crossings etc. As a result it

cannot be used to improve accessibility at the larger macro scale.

Problem 2: The Limited Recognition of Accessibility in the Documents Used to Design Pedestrian Infrastructure in Central Wellington.

The second issue is with the main documents used to design pedestrian infrastructure in Central Wellington. These are:

- Wellington Walking Policy

(WCC Wellington Walking Policy)

\section{- The Pedestrian Planning and Design Guide}

\section{- $\quad$ Wellington District Plan Guidelines}

(WCC Wellington City Council District Plan)

The documents demonstrate a poor understanding of how pedestrian accessibility is affected by the built environment. This is evident in:

- $\quad$ The limited variety of pedestrian access typologies recognised in the documents;

- Secondly, when assessing how each pedestrian typology affects accessibility, the documents fail to recognise all of the criteria that make up accessibility from the travel behavioural theory.

I argue that this is significant because it reduces how effective current improvements to the pedestrian network are towards increasing pedestrian accessibility.

Problem 3: Pedestrian Infrastructural Design and Public Space Design as two Separate Agendas.

The third problem with the current pedestrian process is that pedestrian infrastructural improvements and public space improvements are addressed as two separate agendas, rather than together under one strategy. The benefits of addressing the two areas together are highlighted in 'Design Experiments 3 and 4'. These are:

- $\quad$ New place-making opportunities arise to implement public spaces in the pedestrian network;

- Cost efficiency and the effectiveness of the design response increases. This is because resources are streamlined when pedestrian infrastructural improvements are researched, planned, and implemented, together with public space improvements. 
The 'New Pedestrian Design Strategy': How it can Improve the Problems With Pedestrian Design Practice in Central

Wellington.

This section is broken into two parts:

1. Firstly, a recap on how the New Pedestrian Design Strategy' was developed in this thesis.

2. Secondly, how this new strategy could improve current pedestrian design practice in Central Wellington, if it was adopted by authorities.

1. Developing the 'New Pedestrian Design Strategy'

The initial 'Hypothesis Pedestrian Design Strategy' was designed to address the three problems, outlined in the previous section, regarding Central Wellington's pedestrian design process. These problems were:

1. The techniques used to identify pedestrian accessibility barriers.

2. Limited recognition of accessibility in the documents used to design pedestrian infrastructure in Central Wellington.

3. That currently both pedestrian infrastructure and public space improvements of the built environment are addressed under two separate agendas.

The 'New Pedestrian Design Strategy' is the subsequent outcome of pursuing these problems over the course of the four design experiments carried out in this thesis. Below is a brief outline of how the 'New Pedestrian Design Strategy' evolved through the design experiments.

\section{Typological study and cognitive mapping technique}

- To begin with a 'top down' typological analysis of pedestrian accessibility in Wellington Central was carried out. Also at the same time, a new 'bottom up' cognitive survey method was developed for identifying accessibility barriers in the pedestrian network.

- Both techniques were combined into one 'top down' expert and 'bottom up' qualitative approach for researching accessibility barriers on site.

\section{Design Experiment 1}

- Experiment 1 tested how the typological study could be used as a method of understanding why accessibility barriers occur within the built environment.

- The experiment highlighted that pedestrian accessibility barriers cannot be totally understood through a typological analysis, as the typologies are limited to only understanding accessibility at the micro scale.

- As a result the need for further accompanying 'top down expert' macro scale site analysis was highlighted, in order to understand accessibility in the greater urban environment. 


\section{Design experiment 2}

- 'Experiment 2' tested to see if a standardised typological approach could be used to efficiently (at a low cost) design and resolve accessibility barriers on site. The experiment found two limitations when using a typological approach to designing:

Firstly, the designer needs to be familiar with more than just each accessibility barrier and its accompanying typology within a 'barrier hot spot'. Instead the designer must be familiar with the wider surrounding area, in particular the accessibility barriers along the other possible routes through the site. This is because sometimes it was more effective to spend resources on an alternative route through the site, rather than the original barrier hot spot, as it had, fewer, and more easily resolved accessibility barriers.

Secondly, the standardised typological method of designing failed to recognise the potential pedestrian design interventions have for place-making in the urban environment. This is because typologies fail to respond to the surrounding context, and are isolated simplifications of complex real world problems. As a result opportunities were lost to improve the quality of public space in the built environment, thus highlighting the consequence of using typological solutions.

\section{Design Experiment 3}

- In response to 'Design Experiment 2', 'Design Experiment 3' introduced that designing for place-making was important as it improved the 'sense of place' qualities of the urban environment. The experiment found that new areas of place-making potential could be discovered and developed during the process of resolving accessibility barriers in the pedestrian network.

- And in addition to this uncovered the problem that pedestrian accessibility across the wider pedestrian network cannot be resolved entirely through intervening at a micro scale in the 'barrier hot spot' alone. Instead accessibility and place-making needs to be considered in the macro scale within the wider context.

\section{Design experiment 4 (The final design process)}

- 'Design Experiment 4' combined what was learnt in the previous three design experiments into one complete strategy for researching, designing, and implementing pedestrian infrastructure. Importantly, it also used an additional analysis of the wider urban context to ensure pedestrian accessibility and place-making improvements being made at the micro scale were going in the same direction as of the larger macro scale agenda. 
The experiments found that 'New Pedestrian Design Strategy' used in 'Design Experiment 4' was the most effective and efficient approach for implementing pedestrian infrastructure. This is because the strategy successfully applies a range of 'top down' and 'bottom up' approaches, therefore creating a stronger more efficient method for understanding and resolving behavioural problems, particular in a limited funding economic environment.

\section{Improving the Current Pedestrian Design Process in Central}

Wellington.

The following points will briefly outline how the 'New Pedestrian Design Strategy' could improve current pedestrian design practice in Central Wellington, if it was adopted by authorities.

Success 1: The Combination of Standardisation and Customisation in the Design Process.

The 'New Pedestrian Design Strategy' achieves the right balance between design standardisation and design customisation. In turn this allows pedestrian accessibility solutions to be implemented in the built environment in a cost efficient manner, whilst also exercising the principles of place-making, by being responsive to the surrounding context. For example, this proved most successful in 'Design Experiment 4 '. By being aware of the place-making areas throughout site, and whether there was a requirement for an intervention in these areas to be responsive to their surroundings, the design process could employ a customised design in response. On the other hand, this allowed for standardised typological solutions to be applied to the remaining areas of site cost efficiently, as it had been established there was no consequence in inserting a standardised design that was not responsive to its context.

Success 2: Designing for Accessibility and Place-making Under one Framework

In 'Design Experiment 4' the 'New Pedestrian Design Strategy'

demonstrated that researching, designing, and planning for both:

- Pedestrian accessibility in the built environment,

- $\quad$ And the place-making qualities of public space in the built environment,

could be made more cost efficient if they were combined together under one strategy; thus allowing both agendas to be addressed at the same time through one solution. This approach therefore is successful in two ways:

- $\quad$ Firstly a combined design response is more effective in improving place-making in the pedestrian network. This is because new opportunities arise for developing areas of place-making into the built environment when both issues are addressed together. For example the canopy shelter intervention in 'Design Experiment 3' pg109. 
- Secondly, there is an economic benefit because time and resources are streamlined from two separate areas into one. This was evident in 'Design Experiment 4' when planning the overall framework for addressing both, the accessibility barriers, and place-making areas on site. When these agendas were addressed together the framework ensured the designs did not conflict, but instead benefited from one another. The strongest example of this was when a proposal for a new public space area was strategically planned to be developed along a well used but amenity neglected pedestrian route (Vivian St connection Figure 53 pg130). The planning of this intervention was successful as the new development was also able to fill a large public space void in that area of the public space network (Figure 50 p127).

- Thirdly, addressing both agendas simultaneously makes it easy to develop a long-term framework for implementing both cost efficiently. This was demonstrated in 'Design Experiment 4' which used the hierarchical framework (Figure 60 p137) as a guide specifying the level of urgency for each design to be implemented. This kind of framework was successful because it was responsive to whether the overarching urgency was on:

- $\quad$ Resolving the accessibility barriers in order of barrier significance or,

- $\quad$ Cost efficiency, in terms of having the most affect by using the least amount of resources.
Success 3: The Combination of 'Top Down' Expert and 'Bottom up' Qualitative Approaches to Site Analysis.

The new strategy uses a combination of 'top down' expert and 'bottom up' qualitative approaches to carry out site analysis, because of this; it has significant advantages over using either approach in isolation.

On one hand the 'bottom up' approach to site analysis has two benefits:

- Firstly, it allows authorities and design professionals to learn how members of the public perceive walking in the built environment. This improves both cost efficiency and effectiveness of the design response, as resources can be used more accurately when designers know where the most significant accessibility barriers are in the pedestrian network, and what needs to be addressed.

- Secondly, the same benefit as above applies in terms of identifying areas of place-making potential in the pedestrian network.

However, despite these benefits, the 'bottom up' process is problematic when used as a stand-alone approach. This is because the results from the cognitive survey are not entirely representative of the problems within site. There are number of reasons for this:

- Firstly, the survey is refined to a limited site and thus loses sight of other larger urban factors. This means some areas identified by the cognitive surveys, that seem in urgent need of attention, may 
turn out to be less significant when placed in context among larger site systems, or alternatively a city's long-term strategic plan.

- Secondly, the findings are subject to the limitations of the survey method. For the cognitive survey method this limitation is that the surveys can only be used to identify accessibility barriers in areas of site where the respondents have walked through previously. There is a consequence for this when the data is processed in that some areas of site look as though they have no barriers. However this is incorrect, as it only appears this way because fewer respondents had in fact experienced walking through that area.

However, the above problems are then resolved in the 'New Pedestrian Design Strategy' because three additional 'top down' expert techniques to site analysis are included alongside the 'bottom up' cognitive survey method. These are:

- The typological study of pedestrian accessibility.

- An analysis of place-making at the micro and macro scale.

- An analysis of the overarching macro scale accessibility conditions in the greater pedestrian network.

The 'New Pedestrian Design Strategy' uses these 'top down' expert techniques in combination with the 'bottom up' cognitive surveys, and thus is successful for the following two reasons:
- Firstly, the typological study of pedestrian accessibility can be applied to areas of site where the cognitive surveys could not be used. These are the new or underused areas of site which fewer people have experienced walking through. The typological study is useful because it can be used to highlight the accessibility barriers that are likely to present in these areas, and as a result which barriers a designer needs to consider in their design response.

- Secondly, when researching site, the combination of micro scale and macro scale analysis allows the 'expert designer' to understand how barrier hotspots at a micro scale, such as the areas of place-making potential within the 'hot spot', sit within the larger macro scale environment. This is important when trying to improve the overall pedestrian network for two reasons:

- It ensures all the micro scale interventions are integrated into the broader macro scale framework.

- It allows the designer to be aware of the other accessibility barriers present through site. Therefore when designing a response, this allows the designer to select the route which is most effective.

As a final point this section has highlighted that using a multiple combined approach of a:

- 'Top down' expert and 'bottom up' qualitative approaches to site 
analysis at both a micro and macro scale.

- Standardised typological design approach with a customised design approach.

- Designing for pedestrian accessibility at the same time as developing opportunities for place-making;

makes for a successful strategy for designing researching and implementing pedestrian infrastructure.

\section{(Part 2) 6.2 Typologies and the Architecture and Urban}

\section{Design Professions; and Other Contributions to}

\section{Contemporary Practice}

The second contribution of this thesis to the architecture and urban design professions is in highlighting the benefits and limitations of using typological models to understand and resolve complex environmental problems.

\section{Limitations}

This thesis found that when researching and designing complex environmental problems, a typological approach could not be used as a stand-alone method. This is because typologies are an abstract simplification of a spatial problem, which makes them disconnected from the complex matrix of interrelated spatial problems that exist in the real world environment. The process of simplification behind a typological approach is problematic in two ways:

- On one hand simplification makes a typological approach to site analysis a useful theoretical tool for understanding, in isolation, the spatial principles behind a real world problem. However, the consequence of simplification is that it dissolves the complexity of a spatial problem. Simplification through a typology is a process of finding commonality by stripping away the unique contextual qualities of a problem or site. As a result simplification makes the 
typological method problematic as a method of site analysis, because it is unable to explain complex real world environments without an overarching traditional form of site analysis.

- Secondly, regarding standardised typological solutions as a method of designing, the initial simplification of a typology causes the subsequent typological solution to have little response formally, or aesthetically, to the surrounding environment. For example in 'Design Experiment 2', this is clearly demonstrated by the difference between the standardised typological interventions produced on pg89; in comparison to the place-making designs in 'Design Experiment 3' pg113, that were responsive aesthetically and formally to their surroundings. In a real world example, this is also evident with Wellington's Adshell bus shelters (Figure 23 pg83), whereby the same modular steel and glass design is transplanted in the context of the residential suburbs, as well as the CBD.

As a consequence this thesis argues 'standardised typological solutions' should not be used for resolving accessibility problems in places where the following spatial characteristics can under no circumstances be compromised:

- $\quad$ Character areas, where formal and aesthetic response to site is important.

- Complex areas, where many additional spatial problems are present in the immediate surroundings.

- Place-making areas, where a customised design that responds to the needs of the unique place-making conditions, is required.

These characteristics should not be compromised as they are the most valuable/valued qualities of the site.

\section{Benefits}

Despite the previous criticisms this thesis demonstrates that there are benefits in using a typological approach as a method of researching and designing pedestrian infrastructure.

- Firstly, typologies are a spatial medium that bridges between theory, in a transcript medium, and real world practice in a spatial medium.

- Secondly as a method of analysis they offer a useful technique for interpreting complex spatial environments through a specific theoretical lens. This was evident in this thesis through the typological study of pedestrian accessibility (Appendix A pg194), which played an important part in understanding the spatial conditions that were causing the accessibility barriers to occur in areas of site where the cognitive survey could not. Therefore, typologies in the form of a typological study are significant to pedestrian design practice, because authorities can use them to learn how the public understand pedestrian accessibility in the 
built environment. This must be achieved by developing a

typological study based on the publics' perceptions of accessibility barriers in the built environment.

- Thirdly, standardised typological solutions are an efficient and low cost method of designing. This is because a standardised solution results in a design that has multiple applications yet requires fewer design resources. 'Design Experiment 4' demonstrated this by resolving a range of pedestrian accessibility problems on site, simply by inserting a standardised typological design without requiring much further customising of the design.

- Fourthly, typologies are a useful way of understanding the abstract principles governing a complex environment, however they are not a blueprint solution to a problem. The criticisms and benefits of using typologies as a method of designing highlight a tension between design standardisation and customisation. On one hand typologies are a method of standardisation that offer efficiency benefits through the possibility of re-applying one design solution multiple times. But as a result, typologies are limited in their application as they are inappropriate for resolving sites that have highly valued qualities, and complex spatial problems, which are of vital significance to the accessibility and place-making in the area, and thus cannot be compromised.
In terms of the contemporary theory this thesis has:

- Affirmed Taylor, Zube, and Sell's argument that a multi paradigm 'top down' and 'bottom up' approach for researching and resolving behavioural problems is most effective.

- Provided more insight into the use of typological approaches, as a way of researching through using a 'typological study'; and designing, through using standardised typological solutions.

- Made insightful developments into the use of cognitive survey mapping as a technique for carrying out qualitative perception research of a select survey group, 18-30 year olds. Thus further proving that the cognitive survey method is more cost efficient and effective than the 'on-site' walkthrough Community Street Audit, which is currently used by the New Zealand Transport Agency.

As a final point, this thesis has suggested a whole new way of looking at a precinct. Through analysing something as simple as how pedestrians move through the environment, a designer can achieve two things. Firstly not only can they influence pedestrians' perceptions towards walking, by relieving the most intense moments of accessibility barriers in the city. But secondly, through small interventions such as re-developing a run-down pathway, have the ability to be a catalyst for larger change within the urban environment by influencing the way the pedestrian network functions. 
The aim of this thesis was to improve the current process that Wellington City Council and the Central Government use to design pedestrian infrastructure in Central Wellington. This goal was to be achieved in two parts:

1. By improving authorities' understanding of how pedestrian accessibility is affected by the design of built environment.

2. By demonstrating that new interventions to the pedestrian network, which respond to their surrounding context, create new opportunities for place-making in the built environment. This in turn, can improve the quality of public spaces in the urban environment.

In addition to these goals one further concern of the research was to remain conscious of the limited economic environment that is the reality of pedestrian infrastructural spending in New Zealand.

\section{Conclusion}

Recommendations for Authorities

Having completed four design experiments, each inquiring into an aspect of the thesis aim, multiple conclusions can be made as to how current pedestrian infrastructural design practice in Central Wellington can be 
improved. The new 'Pedestrian Design Strategy' outlined on page 169 reflects these improvements but are listed here as well in two parts:

In order to improve authorities' understanding of how pedestrian accessibility is affected by the design of the built environment, authorities need to:

- Develop from the public's perceptions of pedestrian accessibility, an extensive typological library of pedestrian accessibility in the built environment which consists of both, pedestrian access, and land use typologies.

- Adopt a combined approach of using 'qualitative' cognitive surveys in parallel with an 'expert' site analysis of the wider urban context. Firstly, to uncover how pedestrian accessibility barrier 'hotspots', which are identified through the cognitive surveys, have an effect on the larger pedestrian network. Secondly, to educate designers on what parts of the built environment are causing accessibility barriers in new or unused areas of site, where the cognitive surveys cannot be used.

2

In order to improve the way authorities design and implement pedestrian infrastructure in Central Wellington, authorities need to:

- Bring the processes of researching, planning, and designing, pedestrian infrastructure and public space, together as one agenda. There are considerable advantages of this in terms of improving:

- The quality of public space within the pedestrian network, as public space improvements are able to be integrated into pedestrian infrastructural improvements;

- Cost efficiency through streamlined design and build implementation.

- Use a combination of 'qualitative' cognitive surveys and 'TopDown expert' site analysis, at both a micro and macro scale, to locate places within the pedestrian network that require placemaking and accessibility improvements on the macro scale; in order to ensure micro scale improvements contribute to the overall macro scale direction.

- Design where appropriate using both standardised typological solutions, which are derived from the typological study of pedestrian accessibility, and also unique customised solutions that are responsive to the surrounding context.

- Use a cost versus barrier urgency framework, such as the one developed in 'Design Experiment 4' (Figure 60 p137), to organise into a hierarchical strategy the various stages that the placemaking and accessibility barrier solutions should be implemented within the pedestrian network. 


\section{Last Words}

In conclusion, this thesis has demonstrated how the current methodology for designing pedestrian infrastructure in Central Wellington could be made more effective and cost efficient. In doing so, this thesis has developed a 'New Pedestrian Design Strategy' for researching, designing and implementing, pedestrian infrastructure in the built environment. This strategy is successful because it:

- Overtime will improve Central Government and Wellington local authority's understanding of how pedestrian accessibility it affected by the design of the built environment,

- Provides authorities a cost efficient process for resolving pedestrian accessibility barriers within the pedestrian network, whilst also improving the quality of public space in the built environment.

There are however, a number of limitations to this proposed new strategy:

- Firstly, the typological analysis of pedestrian accessibility was not derived through consultation with members of the public. Therefore this thesis cannot inform authorities of how this process should be carried out in practice.

- Secondly, in order to get maximum cost benefits from the cognitive mapping survey technique, further work is required to eliminate the task of having to manually input the paper surveys into the computer. Developing a computer program is important because it would allow the respondents to draw their cognitive maps directly into the computer and the maps would be automatically organised.

As a final point the title of this thesis asks,

\section{“What does a footpath do?"}

Fundamentally walking is a key part of our daily lives as it is the primary way we as people move through the city. Bearing in mind that the budget for pedestrian upgrades in Central Wellington is extremely low, this thesis suggests a more cost efficient and effective process that not only resolves accessibility barriers to walking, but also acts as a catalyst for the planning and creation of new public spaces within the network. This process will significantly improve the quality of public space within the built environment and make walking a more desirable way of commuting in the city. 


\section{Bibliography}

- $\quad$ Alfonzo, Mariela A. "To Walk or Not to Walk? The Hierarchy of Walking Needs." Environment and Behavior 37.6 (2005): 808-836.

- $\quad$ Appleyard, Donald. "Notes on Urban Perception and Knowledge." Downs, M Roger and David Stea. Image and the Environment Cognitive mapping and Spatial Behavior. Ed. M Roger Downs and David Stea. Chicago: Aldine Publishing Company, 1973. 109-114.

- $\quad$ ARRB Group. Pedestrian cyclist conflict minimisation on shared paths and footpaths. Research rep for Australian Biycle Council. Queensland: Austroads Inc, 2006.

- $\quad$ Auckland City of Cars. Dir. Michael Tritt. Perf. Jan Gehl and Newman. n.d.

- Austroads. Guide to traffic engineering practice: Part 10: Local area traffic management. Austroads Inc, n.d

- $\quad$ Bean, Catherine, Robin Kearns and Damian Collins. "Exploring Social Mobilities: Narratives of walking and driving in New Zealand." Urban studies Journal Limited 45.13 (2008): 2829-2848.

- $\quad$ Beattie, Nick. "Urban Placemaking." Place and Placemaking. Melbourne: People and Physical Environment Reserach, 1985. 13-26.

- $\quad$ Bechtel, Robert B. "The Ubiquitous World of Paper and Pencil Tests." Bechtel, Robert B, Robert W Marans and William Michelson. Methods in Environmnetal and Behavioral Research. New York: Van Nostrand Reinhold Company, 1987. 82-119.

- Cao, Xinyu and Patricia L, Handy Susan L Mokhtarian. "Do Changes in Neighbourhood Characteristics Lead to Changes in Travel Behavior? A Structural Equations Modeling Approach." \ournal of Transportation 34.5 (2007): 535-556.

- Carter, Harold. The Study of Urban Geography. 3rd Edition. London: Edward Arnold Publishers Ltd, 1981.

- Connolly, Peter. Technique: Landscape Architecture Graduate Design Research. Ed. Rene Vander Velde and Peter Connolly. Melbourne: RMIT University press, 2002. 20-32.

- Crane, Randall. "The Influence of Urban Form on Travel: An Interpretive REview." \ournal of Planning and Literature 3.25 (2000).

- Downs, Rogern M and David Stea. Image and the Environment Cognitive mapping and Spatial Behavior. Chicago: Aldine Publishing Company, 1973.

- Downton, Peter. "Knowledge production and transmission." Dowton, Peter. Design Research. Melbourne: RMIT publishing, 2004. 71-90.

- $\quad$ Feimer, Nickolaus R. "Environmnetal Perception: The effect of media, evaluative context, and observer sample." Џournal of Environmental Psychology 4.1 (1984): 61-80. 
- Gehl Associates. City to Waterfront Study. Wellington City Council. Wellington: Wellington City Council, 2004.

- Gehl, Jan. "Three Types of Outdoor Activities and Life Between Buildings." The Urban Design Reader. Ed. Michael Larice and Elizabeth Macdonald. New York: Routledge, 2007. 364-370.

- Gold, John R. An introduction to Behavioral Geogrpahy. New York: Oxford University Press, 1980.

- Golledge, Reginald G. Spatial Behavior A Geographic Perspective. New York: The Gilford Press, 1997.

- $\quad$ GWRC Greater Wellington Regional Council;. "Walking and Cycling Regional Walking Plan." Regional Plan. Wellington: Greater Wellington Regional Council, November 2008.

- $\quad$ Hannes, Els, Davy Janssens and Geert Wets. "Does Space Matter?: Travel Mode Scripts in Daily Activity Travel." Environment and Behavior 41.1 (2008): 75-100.

- Hans L, Westerman. Cities for tomorrow. Sydney: Austroads Inc, 1999.

- $\quad$ Hart, Roger A and Gary T Moore. "The development of Spatial Cognition." Image and Environment. Ed. Roger M Downs and David Stea. Chicago: Aldine Publishing Company, 1973. 246-288.
- Hillman, Mayer. "In favor of the compact city.” Jenks, Mike. The Compact City: A sustainable urban form? London: Routledge, 1998. 36-45.

- Huitt, W. Maslow's hierarchy of needs. 2007. Valdosta State University. 7 Feburary 2012

<http://www.edpsycinteractive.org/topics/conation/maslow.html>.

- Joint Technical Committe LG/2 Road Lighting. "AS/NZS 1158.3.1: 1999,." Road lighting - pedestrian area (Category P) lighting Performance and installation design requirements. Australian/New Zealand Standards., 1999.

- Kaplan, Stephen. "Cognitive Maps in Perception and Thought." Downs, Roger M and David Stea. Image and the Environment. Chicago: Aldine publishing company, 1973. 63-79.

- Kearns, Robin A. "Knowing Seeing? Undertaking Observational Research." Hay, Iain. Qualitative Research Methods In Human Geography. Melbourne: Oxford UP, 2005. 192-206.

- Limanond, Thirayoot and Debbie A Niemeier. "Accessibility and Mode-destination Choice Decisions: Exploring Travel in Three Neighbourhoods in Puget Sound. WA." Jounral of Environment and Planning B: Planning and Design 30 (2003): 219-238.

- $\quad$ Litman, Alexander Todd. "Economic Value of Walkability." Research paper for Victoria Transport Policy Institute. 2011. 
- $\quad$ Lynch, Kevin. Image of the city. Cambridge: Massachusetts Institute of Technology, 1960.

- $\quad$ Marans, Robert W. "Survey Research." Bechtel, Robert B, Robert W Marans and William Michelson. Methods in Environmnetal and Behavioral Research. New York: Van Nostrand Reinhold Company, 1987. 41-81.

- $\quad$ Mees, Paul and Jago Dodson. "Backtracking Auckland: Bureaucratic rationality and public preferences in transport planning." 2006. Griffith University. 2 August 2010 <<http://www.griffith.edu.au/dspace/handle/10072/12666>>.

- $\quad$ Mees, Paul and Jago Dodson. "The American Heresy: Half a Century of Transport Planning in Auckland." NZ Geographical Society/ Aust Inst of Geographers Conference. Dunedin: University of Otago, 2001. 170.

- Mehta, Vikas. "Walkable streets: pedestrian behavior, perceptions, and attitudes." Journal of Urbanism 1.3 (2008): 217-245.

- $\quad$ Miller, L Keith. Principles of Everyday Behavior Analysis. 4th. Victoria: The Thompson Corporation, 2006.

- $\quad$ Ministry of Transport, New Zealand. "How New Zealanders Travel: Trends in how New Zealanders travel." Jun 2009. 6 August 2011 <http://www.transport.govt.nz/research/Documents/How\%20New $\% 20$ Zealanders\%20travel\%20web.pdf>.
- $\quad$ Ministy of Justice. "National Guidlines for Crime Prevetion Through Envirnomental Design." National guidlines. Wellington: Ministry of Justice, November 2005.

- Mondschein, Andrew, Evelyn Blumenberg and Brian Taylor. "Accessibility and Cognition: The Effect of Transport Mode on Spatial Knoweldge." Urban Studies 47.4 (2009): 845-866.

- $\quad$ Naess, Peter. "Accessibilty, Activity participation and location of activities: Explorin the links between residential location and travel behavior." Journal of Urban Studies 43.3 (2006): 627-652.

- Nevid, Jeffry S. Essentials of Psychology: Concepts an Applications. 3d Edition. California: Wadsworth, 2009.

- $\quad$ NZTA New Zealand Transport Agency. "The Pedestrian Planning and Design Guide." Wellington: New Zealand Transport Agency, October 2009.

- $\quad$ Pucher, John. "Rural mobility and mode choice: Evidence from the 2001 National Household Travel Survey." Journal of Transportation 32.2 (2005): 165-186.

- $\quad$ Ramsay, Anthony. "Planning urban networks for walking." Tolley, Rodney. The Greening of Urban Transport. Ed. Rodney Tolley. London: Belhaven Press, 1990. 159-171. 
- Schlossberg, Marc, et al. "School Trips: Effects of Urban Form and Distance on Travel Mode." American Planning Association: Journal of the American Planning Association 72.3 (2006): 337.

- Snellen, Danielle, Borgers Aloys and Harry Timmermans. "Urban form, road network type, and mode choice for frequently conducted activities: a Multilevel Analysis using Quasi-experimental design data." Journal of Environment and Planning A 34.7 (2002): 12071220.

- Soltani, Ali, et al. "Design For Movement: Linking Non-work Travel and Activity Level to Local Urban Design Dimensions." Urban Design International 11.3-4 (2006): 173-186.

- $\quad$ Southworth, Michael. "Designing the Walkable city." \ournal of transportation engineering 131.4 (2005): 246-257.

- $\quad$ Statistics New Zealand. "Commuting Patterns in New Zealand: 19962006. Main means of travel to work." October 1998. Statistics New Zealand. 11 August 2010

$<$ http://www.stats.govt.nz/browse_for_stats/people_and_communiti es/geographic-areas/commuting-patterns-in-nz-1996-2006/carbus-bike-or-train.aspx>.

- $\quad$ Taylor, Jonathan G, Ervin H Zube and James L Sell. "Landscape Assessment and Perception Research Methods." Bechtel, Robert B, Robert W Marans and William Michelson. Methods in Environmental and Behavioral Research. New York: Van Nostrand Reinhold Company, 1987. 361-393.

- $\quad$ Thill, Jean-Claude and Marim Kim. "Trp making, induced travel demand, and accessibility." \ournal of Geographical Systems 7.2 (2005): 229-248.

- VUW, Victoria University Wellington,. Student Life All Shiny and New. 2009. 28 December 2011

<http://www.tepunivillage.co.nz/news/11/student-life-all-shinyand-new.aspx>.

- $\quad$ WCC Wellington City Council. "District Plan." Te Ara Haukawakawaka Design Guide. Wellington.

- $\quad$ WCC Wellington City Council. "Walking Times from CBD using Footpath Network." 2008.

- $\quad$ WCC Wellington City Council. "Wellington Walking Policy." Local Government Policy. Wellington: Wellington City Council, November 2008.

- $\quad$ WCC. "Wellington Walking Policy." Local Government Policy. Wellington: Wellington City Council, November 2008.

- Weiten, Wayne. Psychology: Themes and variations. 7th Edition. California: Thomson Wadsworth, 2007. 
Zacharias, John. "Pedestrian Behavior and Perception in Urban

Walking Enviornments." Lournal of Planning Literature 16.1 (2001):

3-18. 


\section{List of Figures}

Figure 1: NLTF spending on walking and cycling since 2002 (NZTA 49). ... 8

Figure 2: (GWRC Short trip active mode research 12)

Figure 3: Pedestrian catchments (WCC Walking Times Using Footpath Network) 10

Figure 4: Example of a Reinforcer (Weiten 226).. ....16

Figure 5: Map summarising how the design of the built environment affects travel

behaviour...

Figure 6: Hierarchy of Walking Needs (Alfonzo 825).

Figure 7: New Framework for Pedestrian Accessibility. .. .34

Figure 8: How Southworth, Alfonzo, and Metah's, walking models are distributed

in the new framework for pedestrian accessibility in Figure 7. .35

Figure 9: Summarised Table of Southworth, Alfonzo, and Metah's, walking Models.

Figure 10: Representational diagram showing the scope of various pedestrian

design theory and guidelines in Central Wellington.

.......36

Figure 11: Benefits and Weaknesses of Taylor, Zube, and Sell's, four paradigms for

perception research. .. .45

Figure 12: The four Research Paradigms ordered into 'bottom up' and 'top down' approaches 46

Figure 13: Initial 'Hypothesis Pedestrian Design Strategy' ............................................50

Figure 14: (Access typology) example ..........................................................................56

Figure 15: (Land use typology) example .........................................................................5

Figure 16: Two scenarios of a 'residential passageway' typology ................................58

Figure 17: Run down or Character? Some accessibility spatial qualities are

conflicting...

Figure 18: Map showing the outer residential catchments surrounding the CBD (not to scale).
Figure 19: Map showing the main 'accessibility barriers hot spot' after overlapping

all the respondents survey data.

Figure 20: Sample of mapping analysis searching for patterns between

respondents' perceptions of accessibility barriers, and the typologies present within site

Figure 21: Some different ways in which 'eyes on the street' can be obstructed for

a 'urban residential street' typology... 74

Figure 22: Diagram showing a person engaging with typologies on the adjacent footpath .. .77

Figure 23: Adshel's standardised bus shelter design ..................................................83

Figure 24: Typologies within barrier 'hot spot' site.......................................................84

Figure 25: Mapping the pedestrian accessibility barriers vs. typologies - for the

Salamanca Rd barrier 'hot spot'.

Figure 26: Land use typology and associated accessibility barriers identified on site -

Figure 27: Standardised typological canopy shelter solution for the 'urban

residential St' typology.

Figure 28: Standardised typological canopy solution for 'bank wall' typology.......90

Figure 29: Before and after - canopy typological solution for 'Urban residential st' typology...

Figure 30: land use typology and associated accessibility barriers identified on site .93

Figure 31: Standardised typological shelter solution...................................................94

Figure 32: Standardised (CPTED) typological solution for the junction where a side path meets the footpath

Figure 33: Before and after - canopy typological solution for 'vegetation' typology . .96

Figure 34: Diagram showing two different options for introducing shelter along a street. . .99 
Figure 35: Diagram showing the site qualities of a node area that was observed to

have strong public space potential

Figure 36: Qualities of the car park site that mediates between the University and

Salamanca Rd. 103

Figure 37: Areas of place-making potential within the Salamanca Rd site .............109

Figure 38: Material palette within the Salamanca Rd site ............................................110

Figure 39: Design response - 'Lack of shelter from weather' .........................................111

Figure 40: Before -> After ..............................................................................................112

Figure 41: Before -> After ...............................................................................................114

Figure 42: Before -> After ............................................................................................116

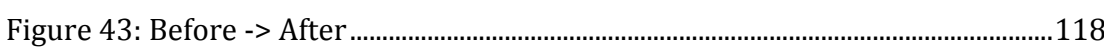

Figure 44: Site boundaries for 'Design Experiments' 2, 3, \& 4...................................123

Figure 45: Design experiment site boundaries..................................................................123

Figure 46: Overlay map of place making potential as perceived by the respondents

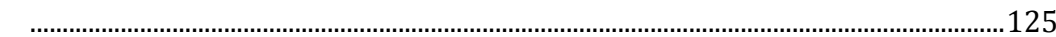

Figure 47: Overlay map of all accessibility barriers as perceived by the

respondents

Figure 48: Map of accessibility barrier hot spots ......................................................126

Figure 49: Place-making qualities on Waiteata rd .......................................................127

Figure 50: Map of areas of place making potential ......................................................127

Figure 51: (Macro scale analysis) - Major pedestrian routes through site and

beyond..

129

Figure 53: Neglected connection to CBD - Vivian St..................................................130

Figure 52: (Macro scale analysis) - Hierarchy of pedestrian volumes.......................130

Figure 54: (Macro scale analysis) - Public space and green space ..............................131

Figure 55: (Macro scale analysis) - Two key nodes in the pedestrian network ....132

Figure 56: NODE 1 - Place-making qualities .................................................................133

Figure 57: NODE 2 Place-making qualities.......................................................................134

Figure 58: Sample artist impression of new public space.............................................134
Figure 59: The barrier 'hot spots' and areas of place-making potential, as identified from the macro and micro scale analysis

Figure 60: Hierarchical framework for implementing pedestrian infrastructure.

Cross referencing - Site conditions / Cost / and Urgency.

Figure 61: Barrier Zone 'A' accessibility barriers and consideration - Eastern end

Figure 62: Barrier Zone (A) - Eastern end New - design proposal.

Figure 63: Barrier Zone (A) Western end - Minor accessibility barriers and considerations

Figure 64: Barrier Zone (A) Western end - Major barriers and considerations...142

Figure 65: Place-making area (3) accessibility barrier considerations ....................144

Figure 66: Barrier Zone (A) - Western end - Proposed solution ................................145

Figure 67: Artist impressions before -> afters

Figure 68: Barrier Zone (B) - Minor \& Major accessibility barriers and

considerations

Figure 69: Barrier zone (B) Retrofit to existing stair treds....... .150

Figure 70: Barrier zone (B) - Standardised typological lighting and shelter

improvements.

Figure 71: Barrier Zone (C) - minor accessibility barriers and considerations......152

Figure 72: Barrier zone (D) - Lighting strategy Before -> After .. .154

Figure 73: Barrier Zone (D) \& (E) accessibility barriers and proposed solution...155 Figure 74: 'Thoroughfare bush typology' CPTED lighting design guidelines .........156 Figure 75: 'Thoroughfare bush typology' CPTED spatial design guidelines ...........157 Figure 76: Barrier Zone (E) - Existing hidden path up to the University - Before ->

After

Figure 77: 'Place-making area (6) - Place-making qualities..........................................160

Figure 78: Place-making qualities of site .......................................................................160

Figure 79: Barrier Zone (F) - accessibility barriers and additional considerations

.. .162

Figure 80: View point ' 3 ' artist impression of standardised typological canopy..163

Figure 82: Barrier zone (F) - Proposed shelter intervention..

....163

Figure 81: View point '2' artist impression of vegetation canopy 
$\mathbf{A}=$ Typological study

Access types

- Hybrid (Access and Land use) types

- $\quad$ Land use types

210

$\mathbf{B}=$ Cognitive survey pilot test

$\mathbf{C}=$ Cognitive survey 1 questionnaire

D = Wellington road hierarchy (obtained from Wellington City Council 2012)

224

$\mathbf{E}=$ Table review of pedestrian design practise in Central Wellington

$\mathbf{F}=$ Cognitive survey 2 questioner

$\mathbf{G}=$ Pedestrian count data (obtained from Wellington City Council 2008) 
Appendix (A): Typological study

(Access typologies) 

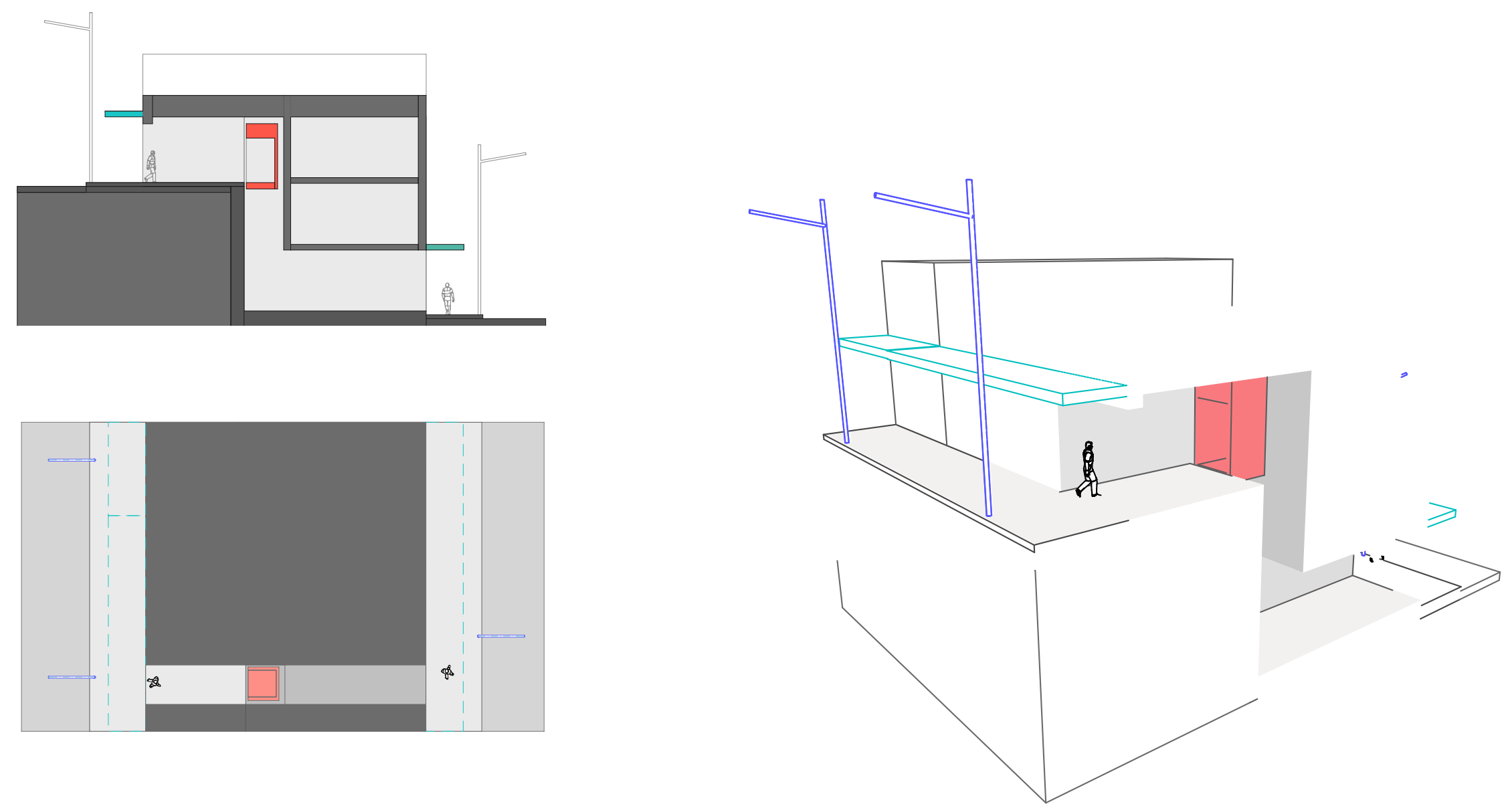


\section{Path - Terrace}

Description

A pedestrian only pathway that is achieved by transitioning between two different street levels. Generally the route calves through uneven terrain, and is a minor route between the

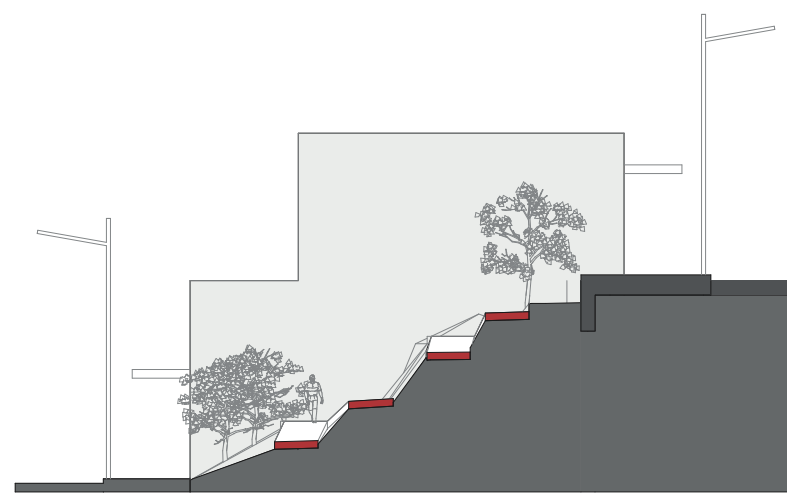

Section AA

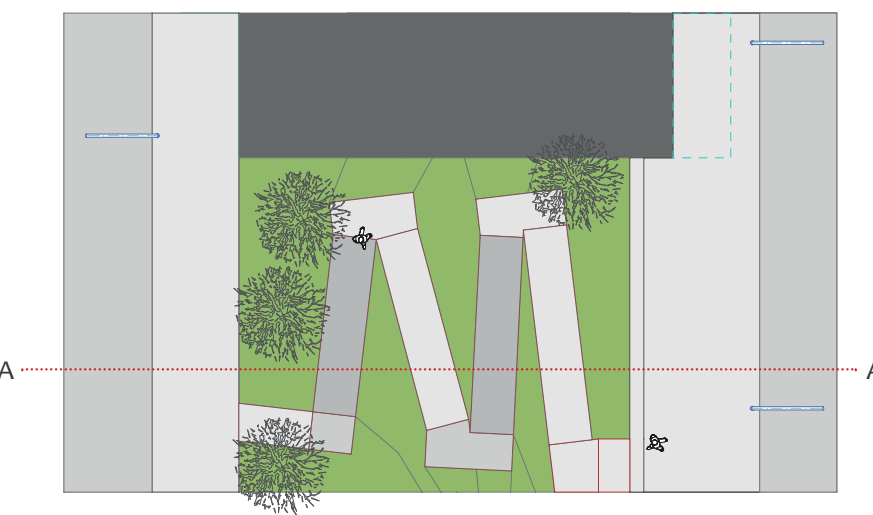

Plan

\section{Safety (CPTED)}

Social safety

Relies on the path provid-

ing visibility or access to

residential houses. Pas-

sive surveillance is much

more likely in this case

than if the path acts as a

thoroughfare only.

Evening Lighting There is often insufficient lighting that creates safety barriers to users in the evening. Additiona footpath lighting other than from the main street is essential.

Spatial design t is important to ensure there are clear sight lines between exit and entry points, and also that users are not hidden from view from the main street. However, this can become an issue if large vegetation is prominent, or bends along the path create poor sight lines.
Proximity \& legibility

- Route continuity

Path quality and steep

rier to less mobile people.

Also safety factors in the

riers to public users.

\section{Route Proximit}

A range of CPTED issues

(see safety category) can

make these paths un-usa-

ble during the evening.

- Legibility

Routes must be sign

posted at street level an

be featured on maps in

order to inform users of

where the path leads to.

This is important when

exit/entry points along

the path are not visible.
Connectivity (Macro)

Characteristics of the

land use pattern

N/A

Treatment of significant

pedestrian barriers

t allows pedestrians ac-

cess through steep sights

that have large height dif-

ferences between streets

at either end.

Connectedness be-

tween paths within the

network

The paths increase

connectivity by adding

fineness to the street

network. Particularly

when multiple paths link

together between build-

ings and houses to creat

fine network of paths.

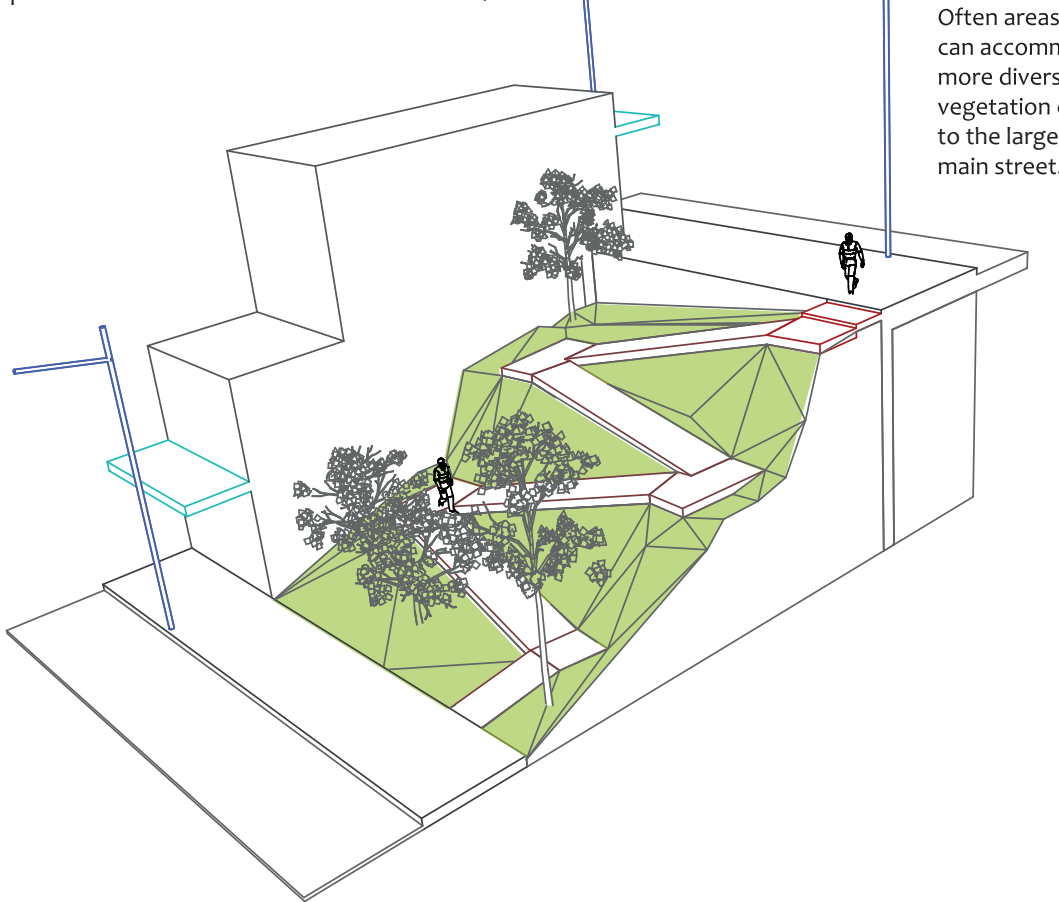

Path quality \& comfort Interest

- Footpath quality

The steepness of the

path and stairs creates a The path provides a much

- Engagement with sur

rounding context

users.

Comfort

Routes are removed from - Social interest

Pedestrian volumes

are dependant on the

Shelter from weather pedestrian network at a

Dependant on tall veg- larger scale

tation and close

ness of buildings.

- Built interest

The path offers an inter-

esting shift in space scale

to that of the main street

network. Also, some

paths may have a signifi-

cant character presence.

Natural scenery

Often areas off the path

a

verse range of

etation compared

trees on the 
Thoroughfare-Bush

Description

A pedestrian only typically dirt/earth track that pass through areas of dense vegetation and trees.

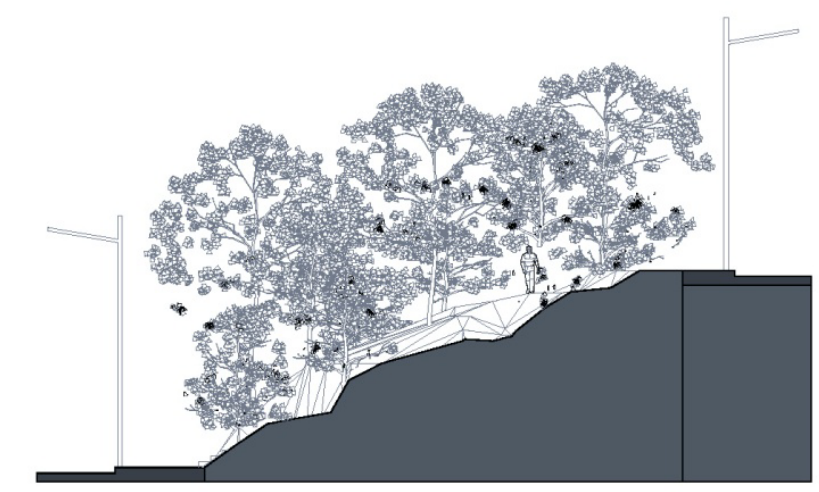

Section AA

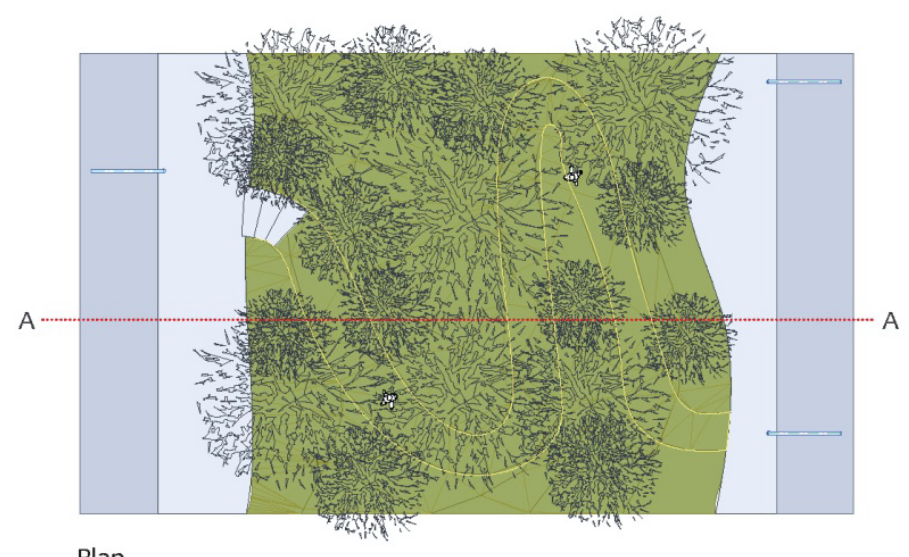

- Social safety less the path is part of a major pedestrian thor oughfare route, users become socially isolated. mobile users due to:

The uneven tracks

- Evening Lighting

Rarely lit making navigation of the path and visual awareness of others dim. weather particul pery.

- Route Proximity The path provides direct pedestrian assess through dense vegetation areas where the main street cannot.

\section{- Legibility}

Relies heavily on being $10-$

catable on a public map,

as well as being signpos

ed to inform the public

where the path leads.

- Spatial design

Dense foliage creates

many hidden areas, and

causes users to become

hidden from view from

the main street. Narrow

paths widths and the

number of path intersec-

options

\section{政

(

Generally after hours un-

Path quality \& comfort

- Footpath quality

Dirt tracks have uneve

surfaces, are often steep,

when wet.

- Comfort

Can be pleasant due to

the full presence of natu-

ral surroundings. How-

ever users can feel isolate

from the surveillance of

the main street.

- Shelter from weathe

Natural canopies offer

shelter from the rain in

places, and dense vegeta

tion shields the wind.
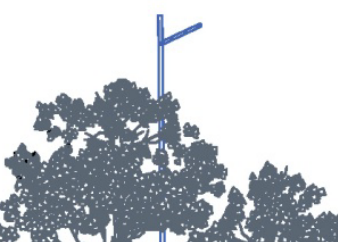

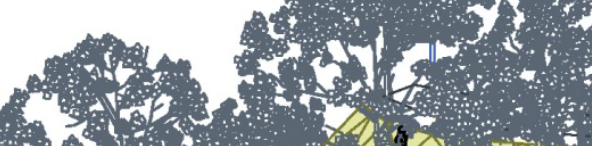

* as

$\sqrt{4}$

$+\div$

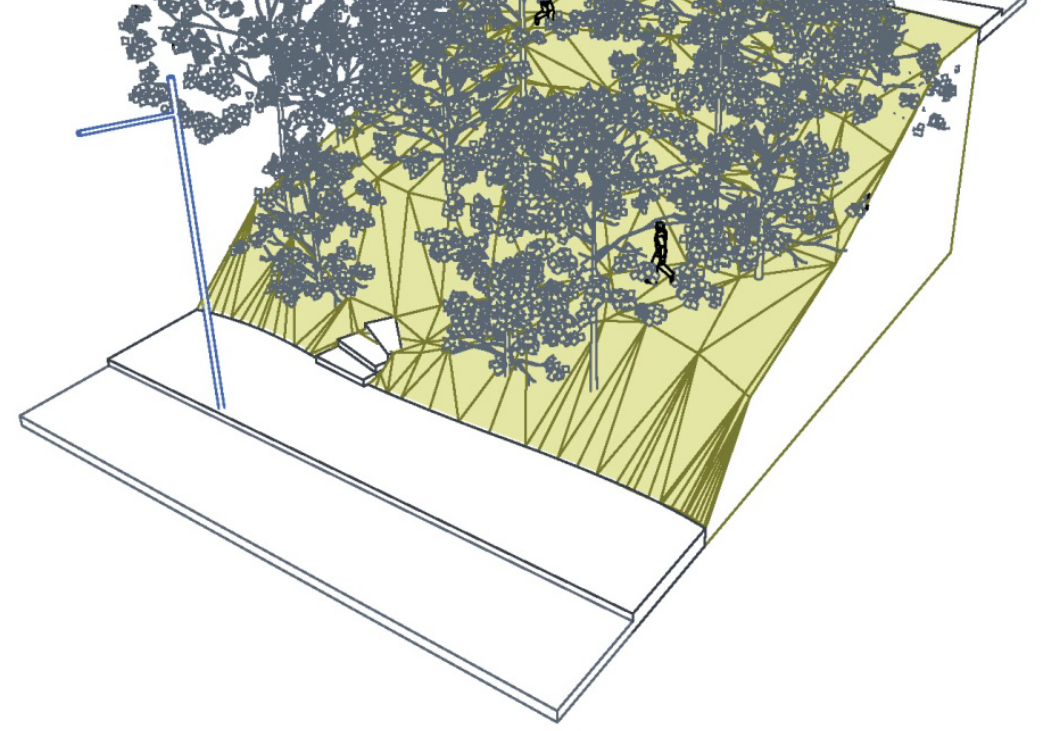

- Characteristics of the

land use pattern:

NA

NA

Building land use

- Treatment of significant

barriers

Allows access through large vegetation areas such as the town belt, banks, gullies etc.

- Linkages

The thoroughfare can

connect the surrounding

St network by providing

access through the physi-

cal barrier of the dens

vegetation.

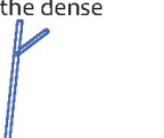

Connectivity (Macro)

Engagement with sur-

rounding context

Very high physical en-

gagement with the

natural surroundings.

vegetation can reduce

vith the greater sur-

roundings.

Social interest

Low, as interaction

only occurs with other

thoroughfare uses. Also

frequency of use will

depend on whether the

path forms part of a key

- Built interest

Only in the case of views

through the vegetation.

No buildings/structures

are present.

Natural scenery

High as users are com-

pletely immersed in the

natural vegetation sur-

roundings. 


\section{Promenade}

Description

A public pedestrian walkway typically running along a waterfront harbour, costal, lake, or river's edge. The path offers

high engagement with the natural surroundings.

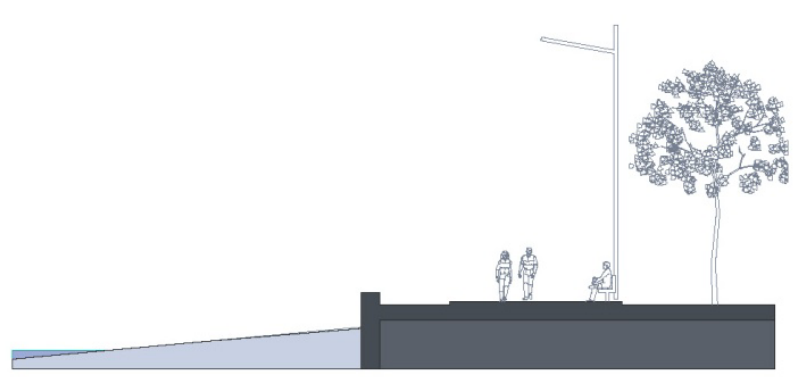

Section AA

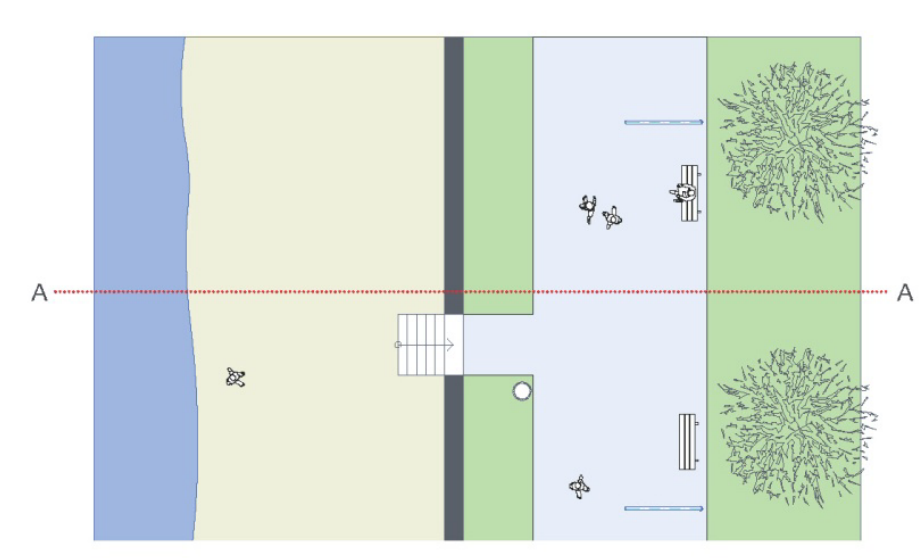

Plan

\section{- Social safety}

The presence of people

can vary dramatically un-

der poor weather condi-

tions if the promenade is

a destination for leisure

activity. On the other

hand when the path

becomes disconnected

from the main street

or a major pedestrian

become isolated after

hours.

- Evening Lighting

Can become a barrier

on less used parts of

the promenade or parts where the path is sepa-

rated from the main pe-

destrian thoroughfare; as

lighting is less prominent.

Spatial design

Landscaping features and

changes in typography

can cause users to be-

from the main st
Proximity \& legibility

\section{- Path Continuity}

Its use is subject to

weather extremes as th

path is completely ex-

posed. But also whether

it is a key commuting

corridor or leisure spot

within the greater pede

trian network; as after

hours safety relies on the

presence of other prom-

enade users.

\section{- Route Proximity}

Promenades may provid

an indirect longer rout

for the user. Thus the

scenic interest qualities

of a promenade present

a trade off to the user

against a more direct

route.

\section{- Legibility}

Dependant on the vis ibility of the promenade

from nearby areas of

the street network. And

also good street signag

including materiality

definition to indicate to

promenade.
Path quality \& comfort

- Footpath quality

Narrow path widths

can become hazardous

during high use when cy-

ers all shear the footpath.

- Comfort

Can be unpleasant under overcrowding when the

path width is narrow an

the space is shared with

cyclists, skate boarders,

and runners.

- Shelter from weather

None due to the absence

of building canopies.

- Noise and air qulaity

The promenade is most

succesful when the foot-

path well separated fro

fast moving and heavy vehicular traffic

acteristics of th

land use pattern

Density

( NA

- Linkages

If a promenades has

potential to become a

commuting corridor that

draws from, carries, and

feeds pedestrians into

other areas of the city.

It is important that the

promenade establishes

good linkages by provid-

ing multiple access point

to key destinations, tow

- n

trian transport corridors.

-Engagement with sur-

rounding context

Very high as users have

free reign, especially

where path access allows

freedom to roam and

physically interact with

the waters edge.

\section{Social interest}

High, under the presence

of leisure activities, par-

ticularly if the pathway

width promotes a variety

sitting to take place.

- Built interest

Subject to the design of

the promenade, presence

of sculpture, landscap-

ing, and surrounding

buildings.

Natural scenery

Dependant on views and ting.

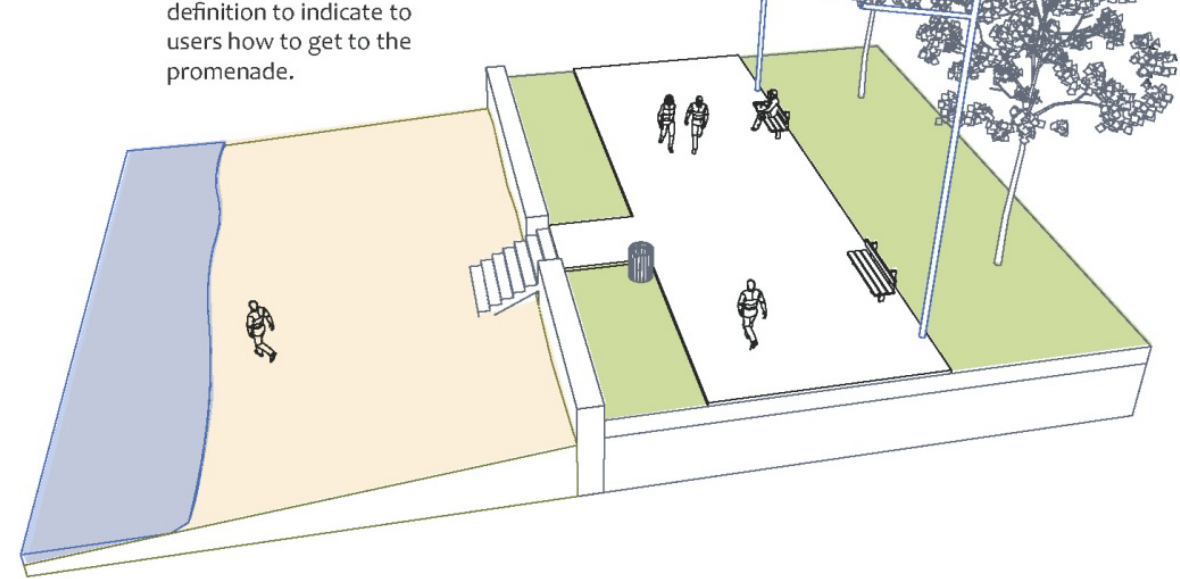


Laneway - Urban

Description

- A secondary pedestrian pathway that passes narrowly between two building structures. Characterised by having

building/store entrances that open out onto the lane way.

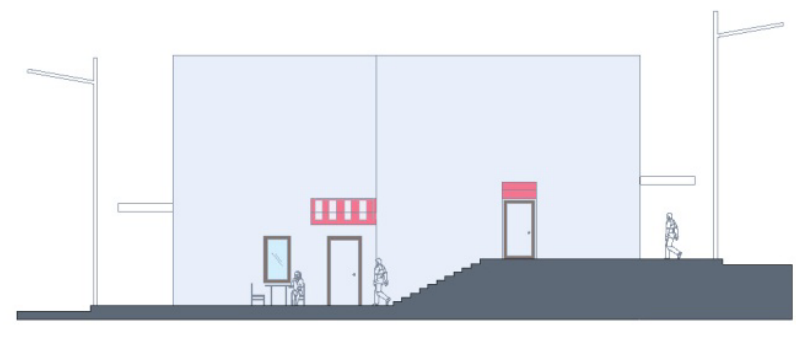

Section AA

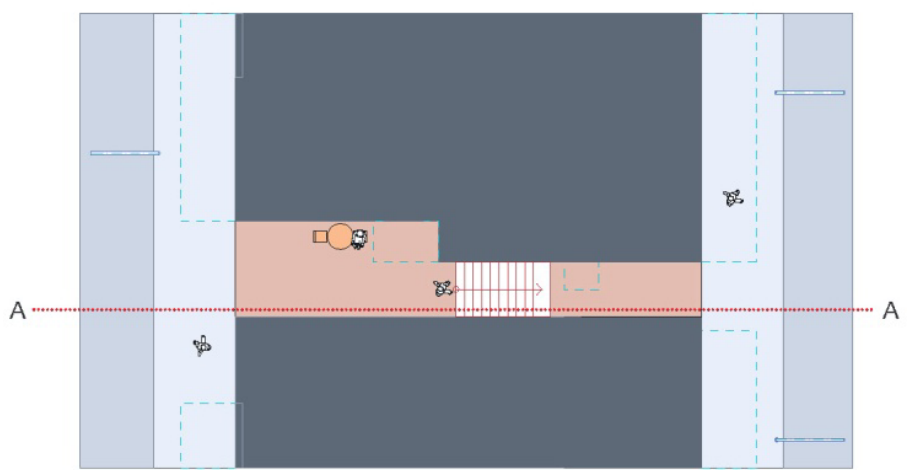

Plan
Footpath quality

Comfort

Pleasant walking enviro ment due to the space

being more personal tha

the main street particu-

Shelter from weathe

No canopies provide she ter from the rain, however some shelter is offered from the buildings either side of the lane which are loser together.
- Social safety

ings are not mixed use,

as in the case with retail

only tenants, laneways

will loose the presence of

- Evening Lighting

Lighting is presen

however the extent can

depend secondary ligh

ceived from stores.

Spatial design

After hours, undulation

in storefront walls and

diversions along the

can create hidden areas.
If the surrounding build-
Characteristics of the

and use pattern

NA

- Density

NA

Treatment of significant

barrie

duces block sizes.

Connectedness be

ween paths within the

network

Networks of laneways im-

prove connectivity by of-

er permeability throug

the building fabric.
In the absence of mixed

use, laneways can be-

come safety barriers to

the public when ground

roor shops close. As no

will be present.

- Route Proximity

Offers a finer route to

that of the larger street

shorter routes.

- Legibility

When visibility between

each end is not possi-

ble. Laneways must be

sign posted to tell users
- Route continuity

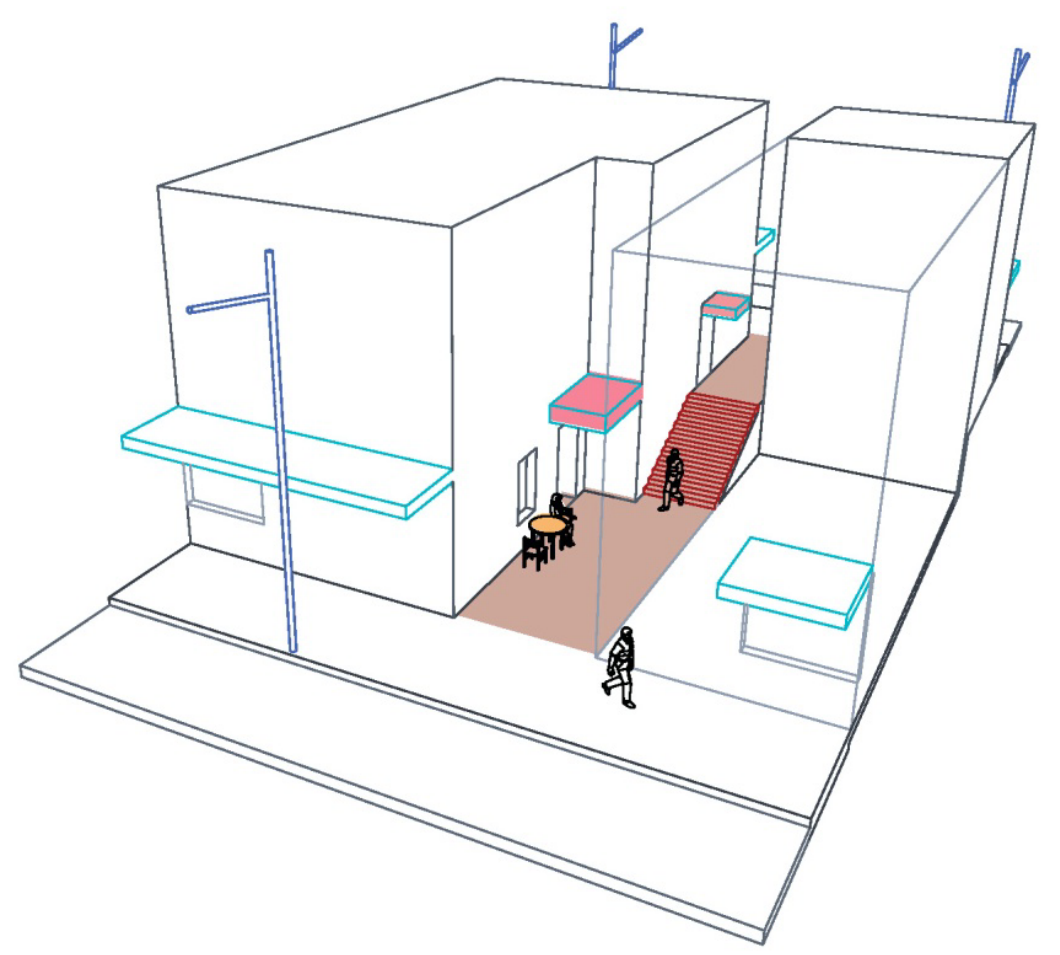

- Engagement with sur-

rounding context

The absence of vehicles

areas physically allows

the users freedom to

Dependant on: the

frequency of shop en-

trances, the transparency

of shop walls promoting

interaction with building

tenants. Whether shop

activity spills out into the

laneway.

\section{- Built interest}

Interest is offered du

to laneways differing

spatially in intimacy, and

often in the variety of

building tenants, com-

pared to the main street

- Natural scenery

In the most part is sub-

ject to landscaping an

planted vegetation. 


\section{Alleyway - Urban}

Description

A narrow path passing between or around the back or sides of buildings. There are no building entrances fronting the alley-

way, however the occasional back entrance might be evident.

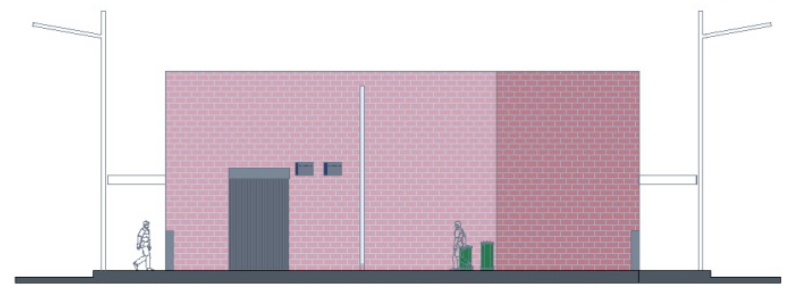

Section AA

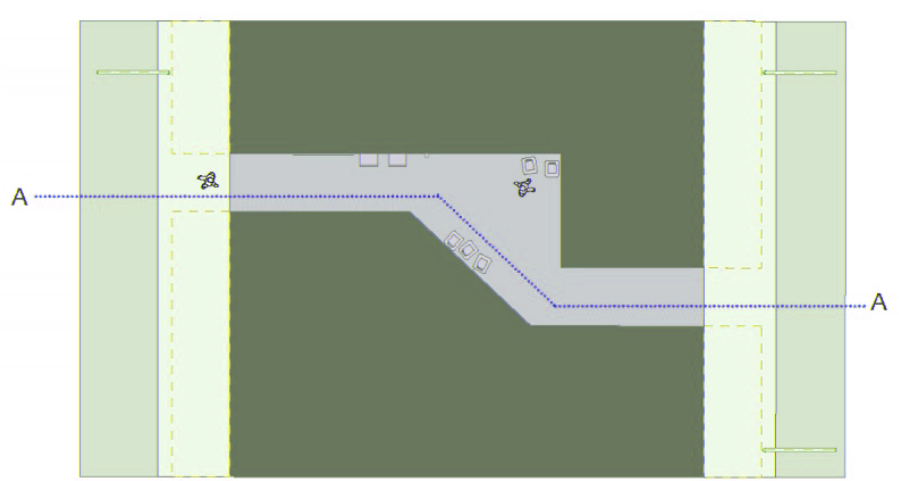

Plan
- Footpath quality

Less maintained than th come hazardos if waste for example is poorly maintained.

\section{- Comfort}

Spacesfeel dirtier due to the presence of waste, mechanical services, and also poorly maintained building walls. due to the narrow space edges of the surroundin buildings, with limited views offered into buildings.

- Shelter from weather No canopies present, however some shelter is ing walls either ing walls either side. way width and building height.

\section{- Social safet}

likely to be a barrier ue to absence of activ ever any social presence depends on the location of the alleyway as a wellof the alleyway as a wellnetwork.

\section{- Evening Lighting} Low, generally insufficiently lit if responsibility resides with the building Frequency

More opportunities in buin whole depth of the section, and the block figur

\section{- Linkages}

Increases the permeability of the building fabric thus improving the connection and route variety between streets.

- Spatial design

Bends along the path and impressions in the builng wals can create Also single entry and exit points offer limited escape routes.

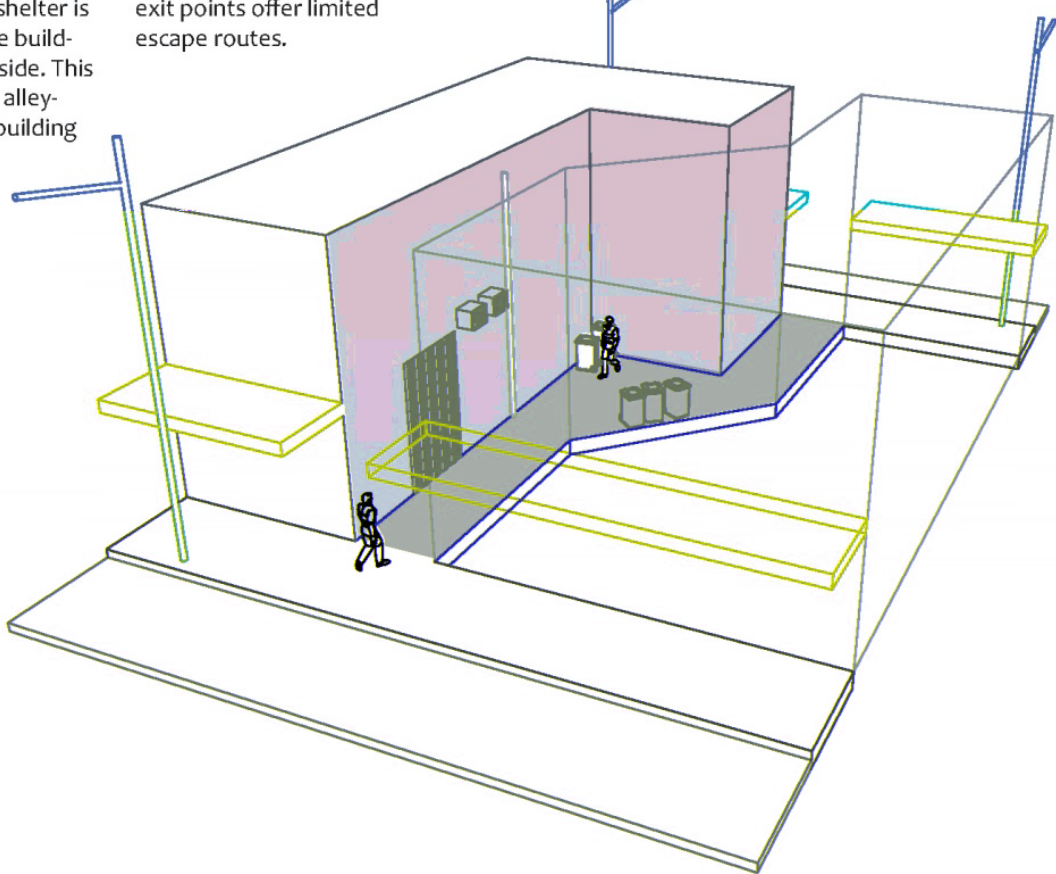

- Route continuity

Safety conditions mak urban alleyways less esrable to use, and subduring the evening.

- Route Proximity

Allows direct movement between the buildings at a finer scale than the larger street network. hours as alls is lost after hours as alleyways are oftenclosed

\section{- Legibility}

Routes are locally known, not always located on a map, and infrequently sign posted.
- Engagement with surrounding context

cilled socially and physically due to the presence no interaction with the building occupants, and building occupants, and

\section{Social interest}

None due to absence of buildings, and therefore social activity, opening

\section{- Visual interest}

Appeals to some user groups as is subject to f the bural expression and character. Street and character. Street art through graffiti and mabricky ar some user groups.

- Natural scenery Only views above to the sky. 


\section{Mall-Internall}

Description

An internal pedestrian street that permits thoroughfare and is typically lined with retail and hospitality tenants. Subject to strict opening hours.

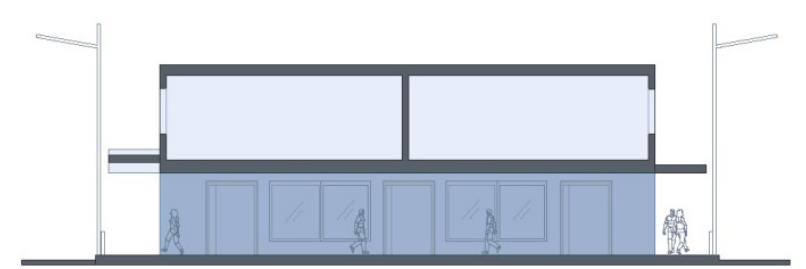

Section AA

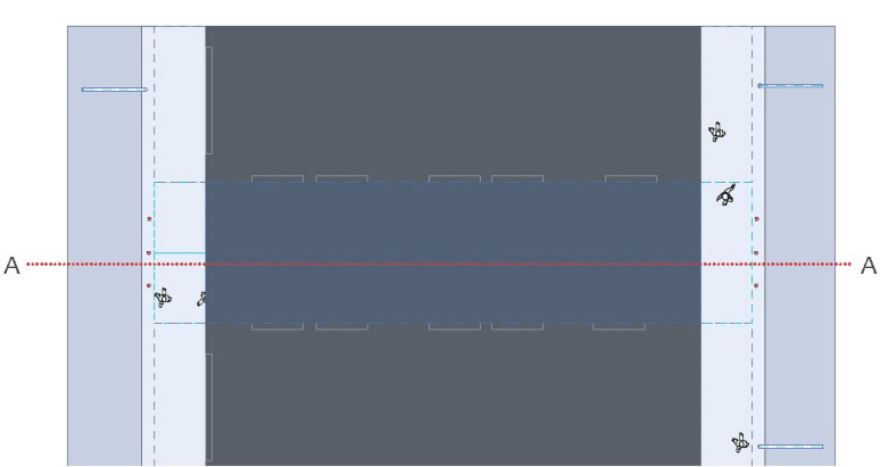

Plan
Footpath quality

Very high as the thor-

oughfare is internal. Also

ramps and stairs etc, are

more functional due to

being subject to interior

building standards.

- Shelter from weather omplete shelter due to the mall being internal.

Buffering from vehicles User comfort is low whe the parking lot is busy due to the lack of pedes. trian right of way, and the danger/ unpredictability of manoeuvring vehicles.

\section{Safety (CPTED)}

\section{- Social safety}

High during mall opening

hours due to the pres-

ance of store work

- Evening Lighting

Highly lit during opening

\section{- Spatial design}

After hours, the buildin

and cannot be used. Thu

safety depends on the

street alternatively use

by the pedestrian. is completely closed off
Connectivity (Macro)

- Characteristics of the

land use pattern:

Density

$\mathrm{NA}$

Building land use

$\mathrm{NA}$

- Treatment of significan

barriers

Reduces block sizes, and

offers a highly safe and

sheltered route.

- Linkages

\section{- Route continuity}

Access is subject to open-

ing hours due to internal

malls being governed by

one larger tenancy.

Route Proximity

Offers a finer route to

that of the larger street

network. Thus creates

shorter routes.

\section{- Legibility}

If the path through the mall is complex or less

obvious, it can be difficul

to identifying that there

through the building.

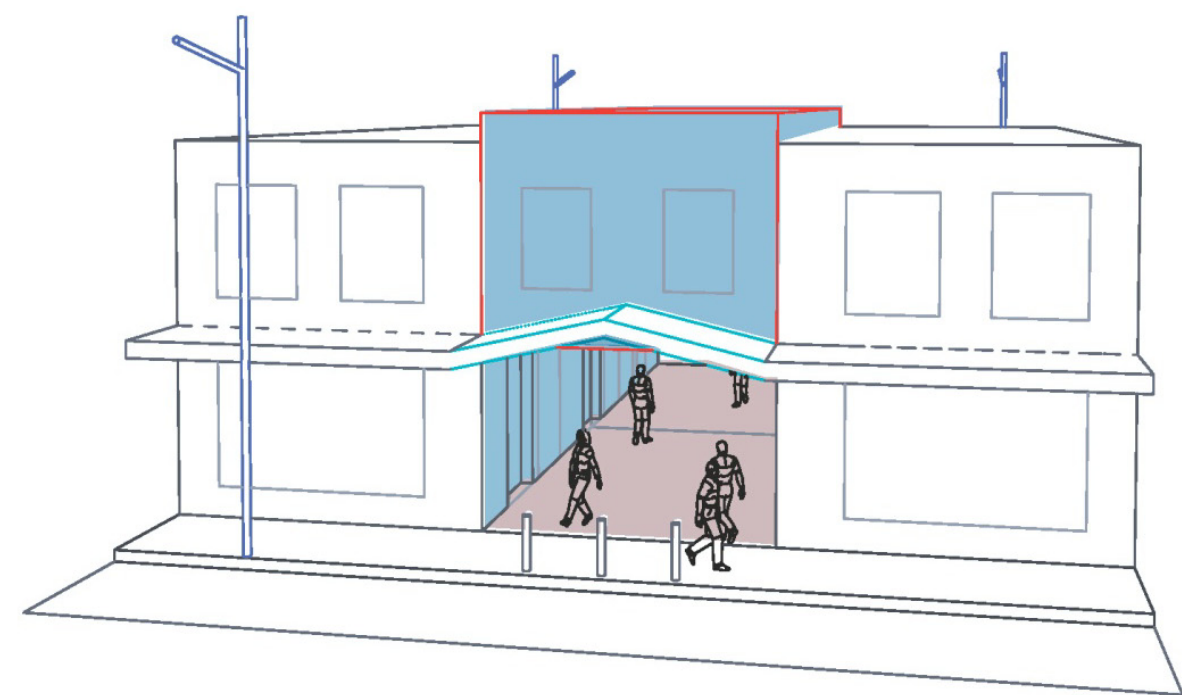

- Engagement with surrounding context

The absence of external views and architectura sensitivity to the surrounding built form, len internal malls to form their own context-less environment, with no engagement with the outside.

\section{-Social interest}

Varies with popularity of the retail/hospitality stores, and also with the ease of 'store user' to

'passer buy' interaction (which depends on tran parency of store walls, and number of sto entrances etc).

\section{Built interest}

Depends on the architec tural expression of the interior. Visual interest is high when store windows are largely glazed and displays are present.

Natural scenery Only in the form planter boxes. 


\section{Service Lane}

Description

- A lane, passing between buildings/sections within a street block, which provides vehicle access to the sides/backs of buildings. Typically the space is used for unloading and loading goods onto vehicles.

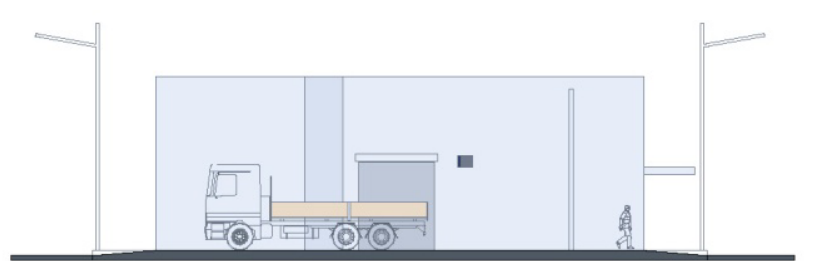

Section AA

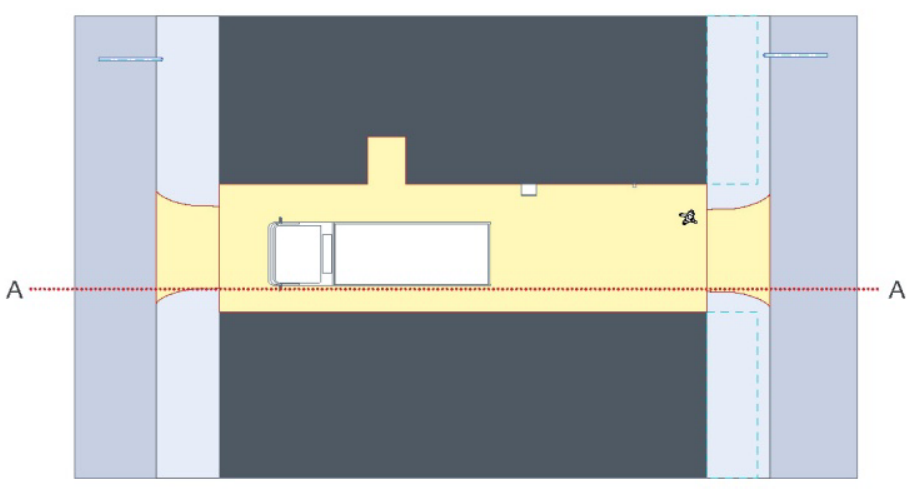

Plan
- Route continuity

Pedestrian barriers can

be created through lack

terest due to poor main-

tenance and the pres-

ence of obstacles su

unloading vehicles and

waste. And also due to

multiple safety factors

especially after hours.

Perception of Proximity

- Legibility

Relies heavily on being locatable on a public map

as well as being signpost

where the path leads.

\section{Social safety}

Low due to absence of

people particularly after

hours as little natural

surveillance as offered

due to buildings having

openings facing the

Evening Lighting

ciently lit if responsibility

Spatial design

Obstacles along the

path and impressions in

ate secluded alcove

Also single entry and

exit points offer limited

escape routes.
Characteristics of the

- Footpath quality

Generally low due to

poor maintenance and

cleanliness as servicing

residing with the building

owner.

\section{Comfort}

Low due to presence

of waste, and mechani-

cal services such as air

there is a high vehicle

priority due to absence of

footpath.

Shelter from weathe

No shelter given from

verandas/canopies.

However some shelter is

possible depending on

the surrounding building

height and closeness to

the pathway.

- Building land use barriers

Reduces bloc sizes

\section{Linkages}

Increases the permeabithus improving the conallowing a shorter route to be achieved. ow, generally insuffi-

building walls can $\mathrm{cr}$

land use pattern:

Treatment of significant ity of the building fabric nections between streets

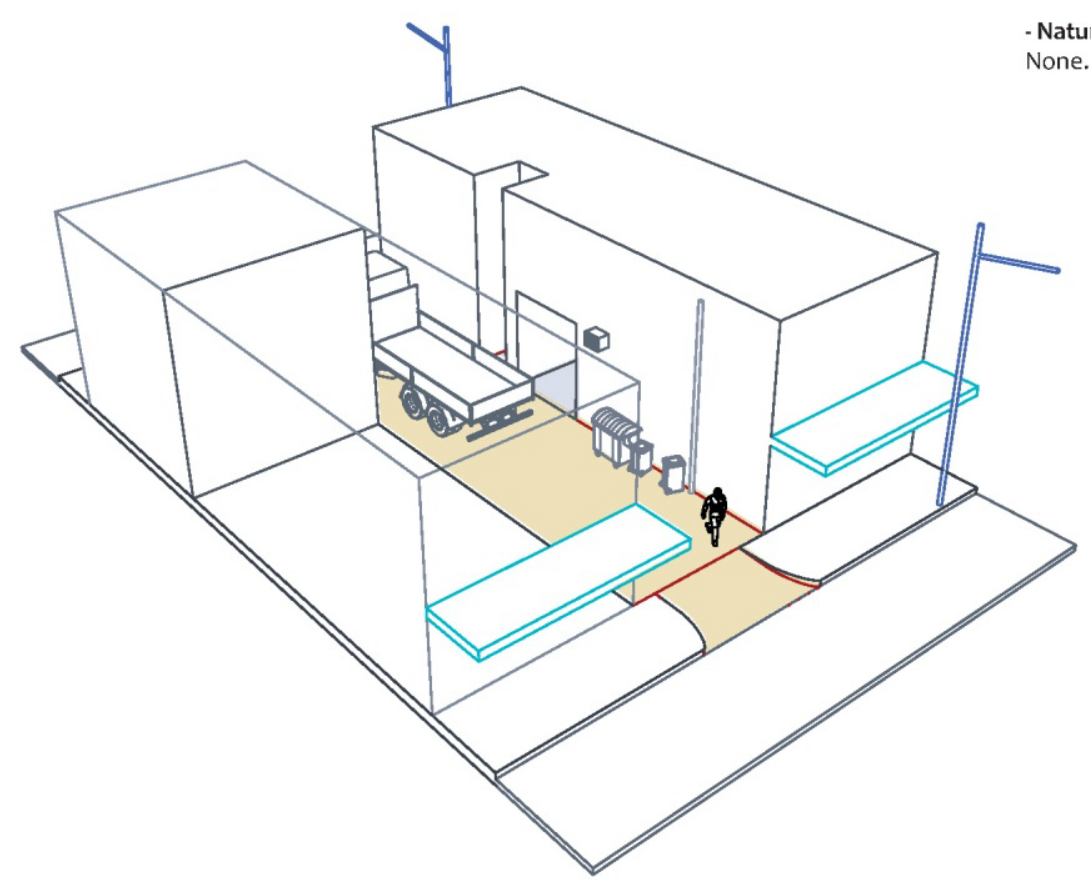

- Engagement with surrounding context

of defined pedestrian

and vehicle areas allows

freedom to navigate the

space. How

engagement is limited

wall openings giving no

wall openings giving no

connection to the inter-

\section{-Social interest}

Only in the case of people unloading vehicles.

- Built interes

Little, however some

building facades can be

appealing such as signifi-

cantly old and deteriorat-

ing brick work.

Natural scenery
Physically, the absence 


\section{Tunne I}

Description

-A completely enclosed pedestrian pathway that passes

through or under a physical barrier such as a road or building.

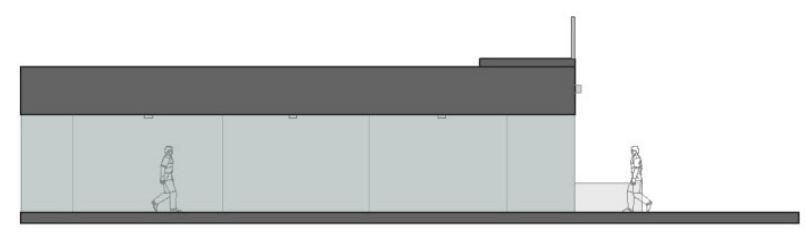

Section AA

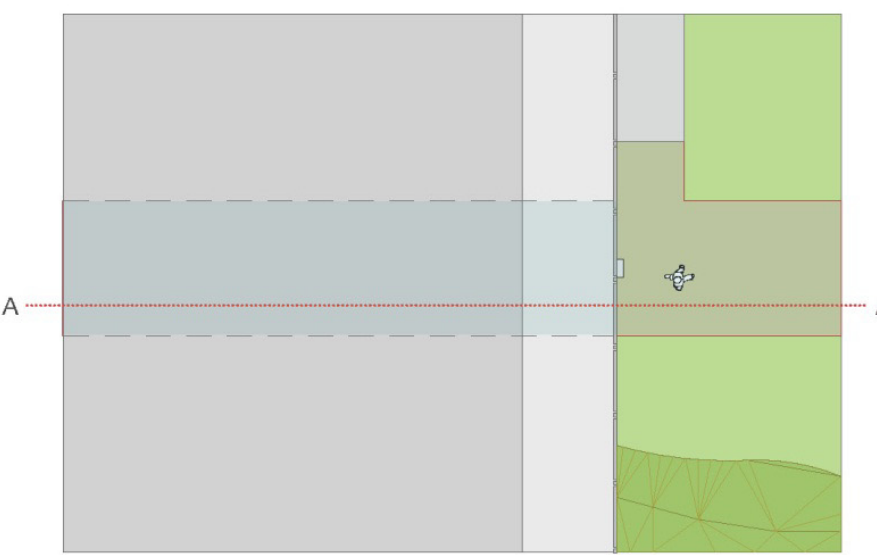

- Route continuity

Tunnels are claustro-

phobic spaces, and can

become psychological

barriers to users. This is

particularly evident after

hours, or where the path

becomes isolated and has

diverged from the main

pedestrian route.

\section{- Proximity}

Travel times can be

reduced where tunnels

pass under and avoid

major roadways, or build-

ing. However conversely

poorly located tunnels

cause pedestrians to

walk inconvenient longer

routes

\section{Legibility}

Located on a map,

however at street level

pecially where the tunn

pecially where the tunn

pedestrian route.
- Social safety

Unless the tunnel forms

art of a significant pe-

pan only

tunnel users.

- Evening Lighting

Cenerous lighting in the

tunnel is essential for

both walking safety and

reducing psychological

barriers.

- Spatial design

The tunnel can be com-

pletely isolated if the

main street. I.e. if the

tunnel level changes rela

Also single entry and

exit points offer limited

escape routes. path is hidden from the

tive to the main street.
Path quality \& comfort

Footpath quality

- Comfort

Quality and cleanliness,

as well as lighting and

'tunnel width to length

ratio', are important in

improving the typically

low comfort.

- Shelter from weather

Completely sheltered.
Characteristics of the and use pattern:

Density

Building land use

-Treatment of significant barriers

or roads, highways, hills,

which in some cases form

the connection between

townships separated by

these significant barriers.

\section{Linkages}

Continue path continu-

ity by allowing physical

access through major

obstructions.
Allows access under ma

Interest

Engagement with sur-

rounding context

None as the tunnel

avoids interaction with

the surroundings.

Social interest

- Built interest

Subject to the architec-

ture and aesthetic of the

tunnel walls.

- Natural scenery

None.

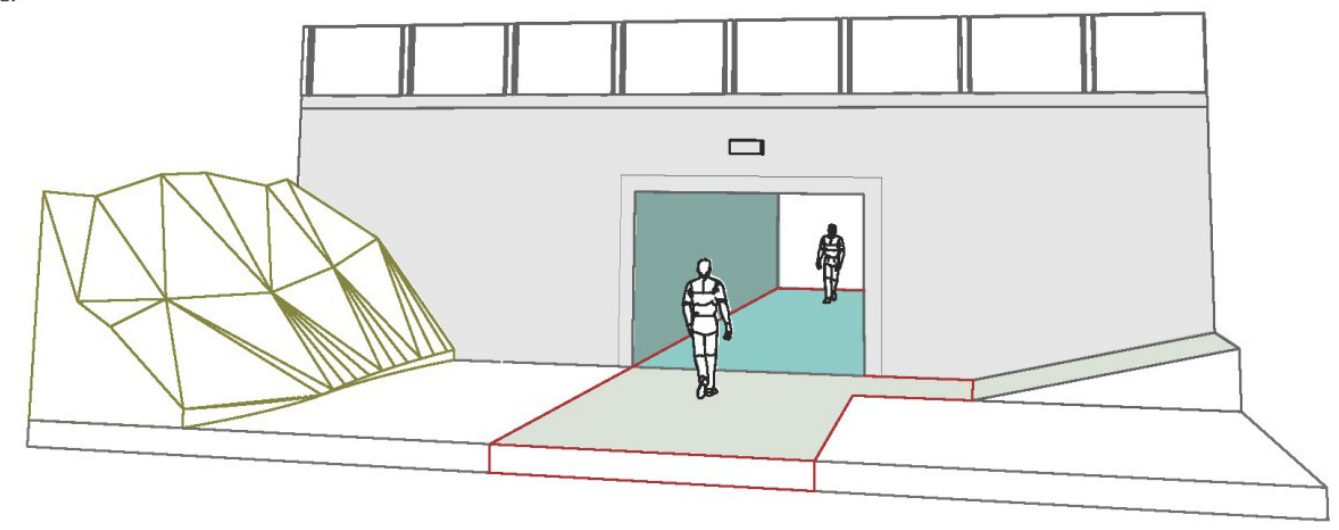

Plan 


\section{Overbridge}

Description

A footpath that passes over a significant pedestrian barrier, i.e. motorway or deep valley, which is exposed due to the absence

of buildings being located between the footpath and bridge

edge.
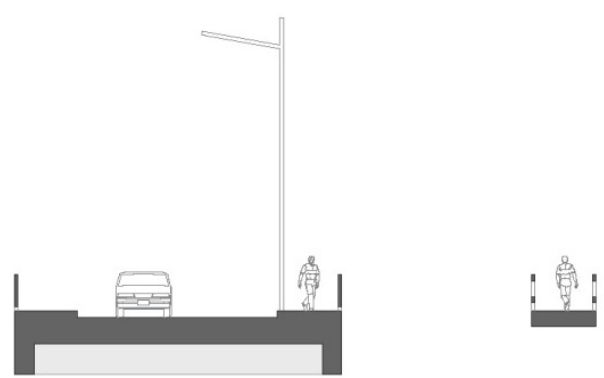

Section AA

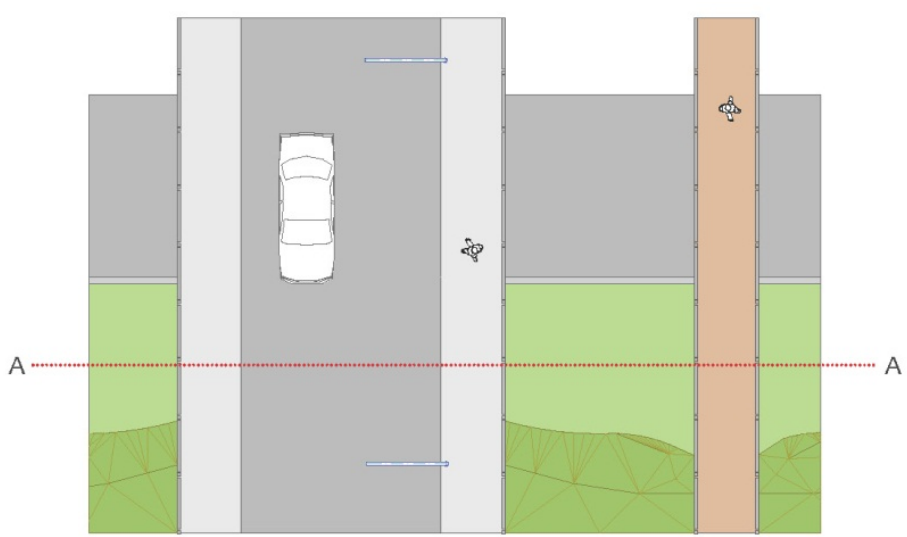

Plan
Safety (CPTED)

Proximity \& legibility

Path quality \& comfort

Connectivity (Macro)

Interest

\section{- Social safety}

Unless the over bridge

forms part of a significant

pedestrian thorough-

Route continuity

Over bridges can become

a barrier under extrem

- Footpath quality

they are totally exposed.

\section{- Comfort}

activity are around due to

the absence of buildings

off the footpath. This is

worse on pedestrian only

Perception of Proximity

re complete-

y exposed. However

Pedestrian bridges over

noise is reduced along

roads have to be raised

no passing vehicles.

causes around level. This

providing wheel chair

access as consueqently

the elevated path.

- Shelter from weather

None as the footpath has

no covering or buildings

Evening Lighting

when: the bridge is pe-

ments

either side.

destrian only, if the path

isolated from the main

pedestrian thoroughfare.

\section{Spatial design}

Narrow path widths

Tall balustrades/wails

can cause users on

elevated bridges to be

hidden from view. Also

undersides of bridges are

places where people can

linger.

igzaging route.

\section{- Legibility}

Overbridges are noticea-

ble from various places at

height.

- Characteristics of the

land use pattern:

- Density

- Building land use

Treatment of significan

barriers

Allows access over major

roads, highways, rivers,

valley, which in some

cases form the connec-

tion between townships

separated by these sig.

nificant barriers.

Linkages

Continue path continu-

access through major

obstructions. ity by allowing physica

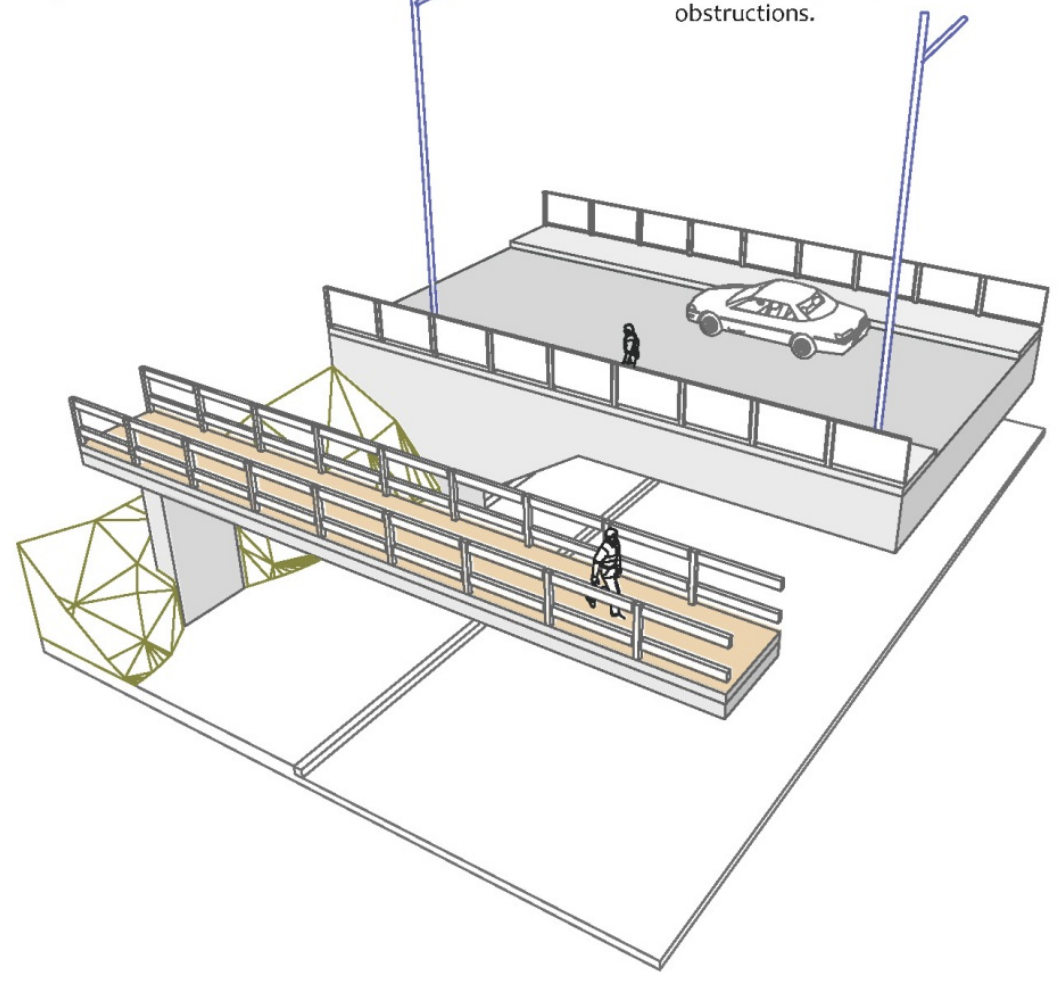

- Engagement with sur

rounding context

Offers an elevated view-

point of the surroundings

environment.

Social interest

Little unless the path is

frequently used, or offers

unique views into/over

Built interest

the over bridge and sur-

rounding buildings.

\section{Natural scenery}

Is dependent on whethe views are offered to the

natural surroundings. 


\section{(Hybrid typologies)}

These fit into the description of both Access and Land use typologies 
Thoroughfare-Parkinglot

Description

An outdoor thoroughfare that passes through an exposed

parking lot, functioning specifically for vehicle use.

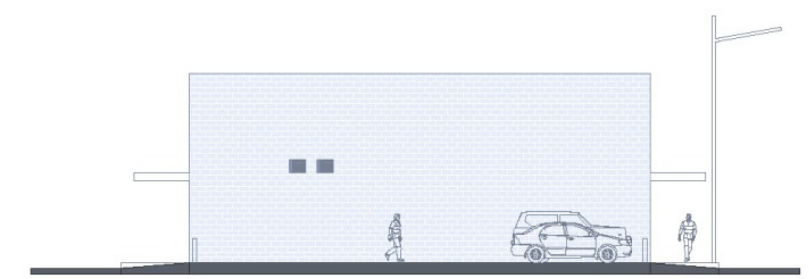

Section AA

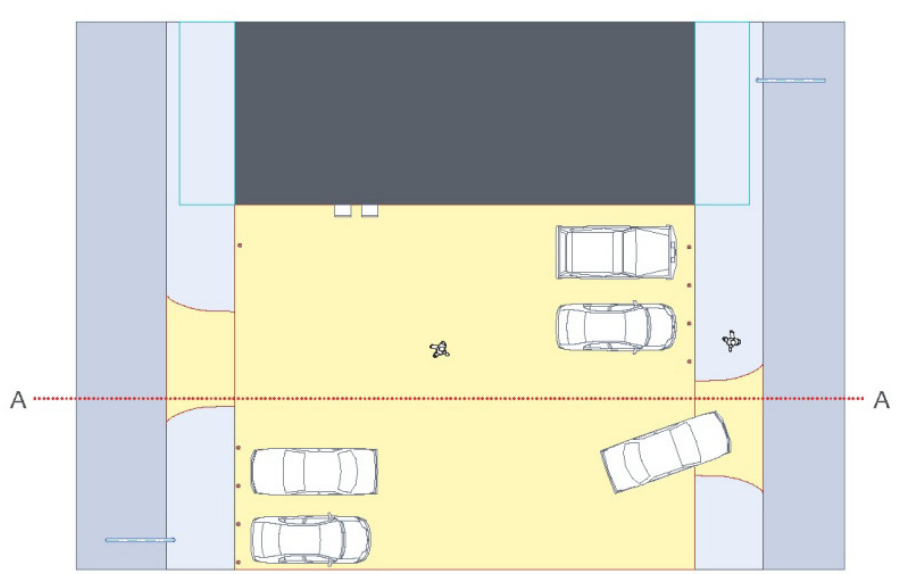

Plan
Path quality \& comfort

- Footpath quality

Varies as maintenance

is subject to the park-

ing lot owner. However

curbs and objects used to

divide up parking slot can

become obstacles that

disrupt pedestrians.

- Shelter from weather

There is no shelter for

users along the footpath

street edge where no

verandas/canopies are

present, as well as when

passing directly across

the exposed car parkin

lot.

Bufferning from vehi-

User comfort is low when

the parking lot is busy

due to the lack of pedes

trian right of way, and

the danger / unpredict-

ability of manoeuvring

vehicles.

A

\section{Safety (CPTED)}

\section{Social safety}

After hours the social

presence may only be

interaction with other car

park users. Depending on

the size of the car park,

users can become isolat

ed as they diverge away

from the street.

Evening Lighting

Lighting varies greatly is

subject to the parking lot

owner.

- Spatial design

During busy hours a haz-

ardous environment for

pedestrian users exists

due to the absence of

clear pedestrian routes.

This forces users to navi-

gate among manoeuvring

vehicles.
Connectivity (Macro)

- Characteristics of the

land use pattern:

- Density

NA

Building land use

NA

- Treatment of significant

barriers

Reduces block sizes by

allowing access between

streets.

- Linkages

NA

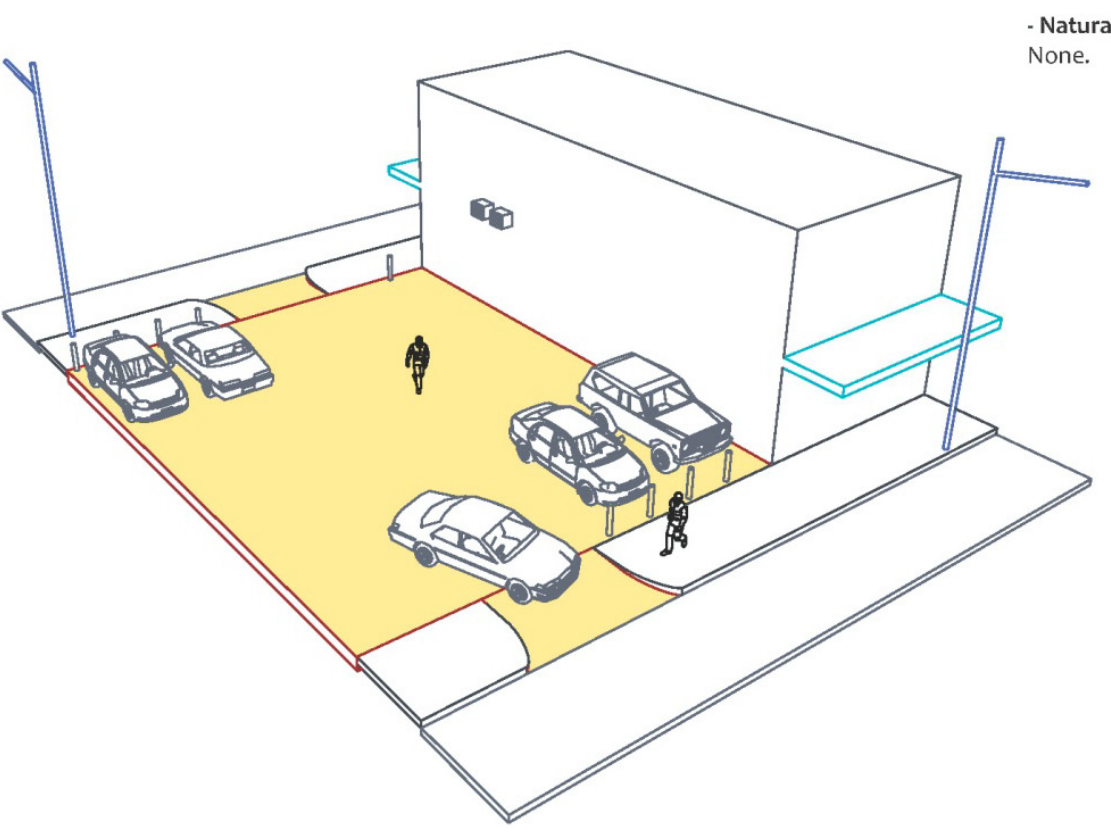

Proximity \& legibility

Route continuity

Lack of shelter can dis-

rupt thoroughfare use

under extreme weathe

conditions.

- Route Proximity

A direct route can be

achieved by navigating

freely across the open

lot. However this de-

pending on the number

cles.

- Legibility

Access through the site

not always identifiable on

a map, however at street

level a short cut may be

much more obvious. car park.

- Engagement with surrounding context

Varies with types of us

ers and business of the

parking lot. For example

engagement is higher

at a supermarket com-

pared to an empty lot, as

when busy there is more

requent interaction with

manoeuvring vehicles

unloading.

Social interest

Interest in the form of

other car park users,

however the level de-

pends on busyness of the

Built interest

nterest through the variety of car types when th parking lot is occupied.

atural scenery 


\section{Path-Field}

Description

- An outdoor space on publicly accessible land ranging from being an exposed field, to having sufficient landscaping, planting and trees. The route can vary from having no pathway, in which navigation is at the discretion of the user, to being completely controlled through landscaping.

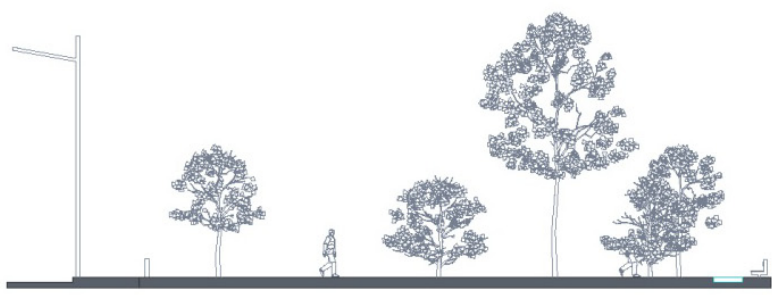

Section AA

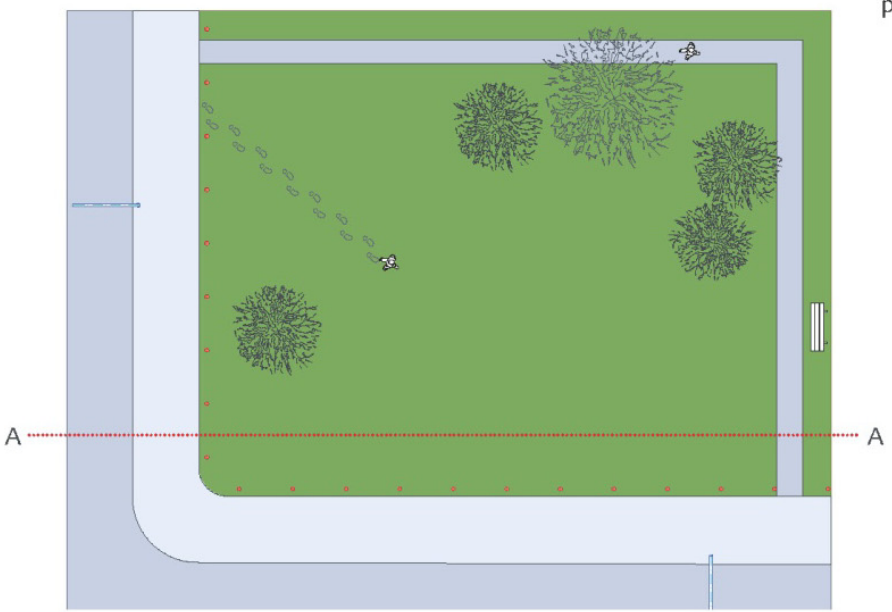

\section{Route continuity}

Lack of shelter can limit

sability under:

xtreme weather condi-

tions; especially where no

path is present and field

become saturated for a

period of time.

And also because of

safety concerns due to

limit use after hours.

Route proximity

Depending of the land-

scape and placement

of footpaths, users can

short cut through the sit

therefore instead of be-

ing refined to the stree

network.

Legibility

Routes are located on a map. However the park/ field can be heavily planted, and have a complex network of paths makin it difficult to judge rout istances, and where paths start and finish.
Path quality \& comfort

\section{- Footpath quality}

Is reduced significantly

the case of an open fiel

where no path exists.

Shelter from weather Is dependant on whether the tenants have covered walkways throughout the site. This is only useful if the tenants are comfortable with members of the public using their facili-

ties.

\section{Comfort}

sers may feel out of

there is a degree of

interaction with the land tenants.

\section{Noise/air quality}

High due to separation

rom the road and users

are emersed in the nat

ral surroundings. place and uncomfortab

\section{- Social safety}

Routes are located on a

map. However the park/

field can be heavily plan

ed, and have a complex

network of paths making

it difficult to judge route

dstances, and where

paths start and finish.

Evening Lighting

Lighting is only on select-

ed paths, and generally

paths are insufficiently lit.

Spatial design

Built landscaping fea-

tures, and dense veg

etation, and typography

an create hidden an

secluded areas.

land use pattern:

Density

- Building land use

Treatment of significant barriers

Alows movement be-

tween the street net-

work.

Connectedness between paths within the

A significant feature to the pedestrian netwo can be created if connects between a series of park/field, within close proximity to each other, link together to form network of parks.

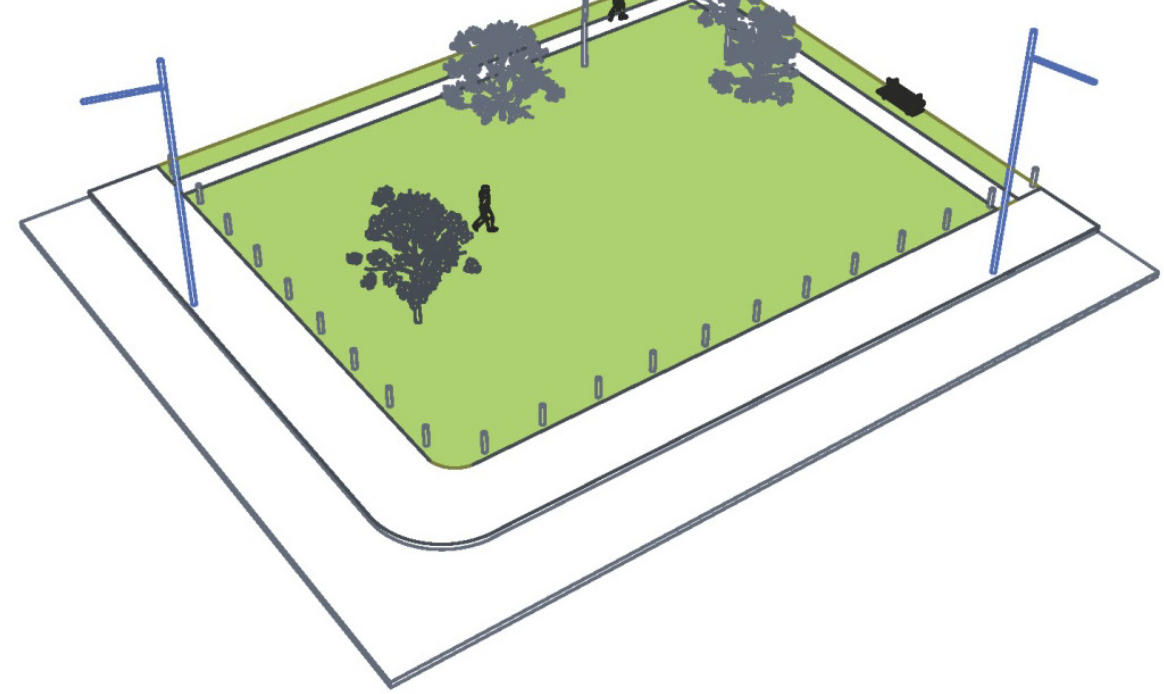

- Engagement with surrounding context In large parks/fields users are given free reign to explore and interact within the setting.

- Social interest

Is weather permitting, but can be very high if the setting is a popular leisure setting/sports fields, or a well used tho oughfare.

- Visual interest

Varies with the variety and amount of vegetation/landscaping as large fields can be mundane for some.

- Natural scenery

Increases when the space develops further than an open field. 


\section{Courtyard/Square}

Description

A large outdoor and informal pedestrian space that permits pedestrian thoroughfare. Typically it is of a paved surface and

surrounded by perimeter buildings on most sides.

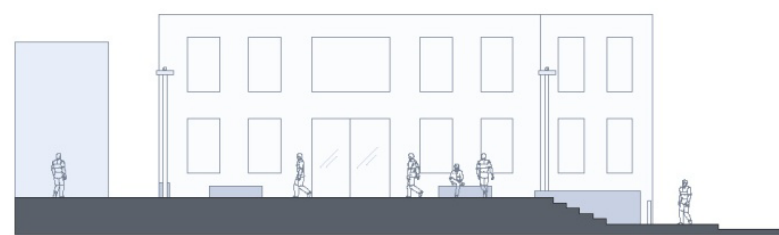

Section AA
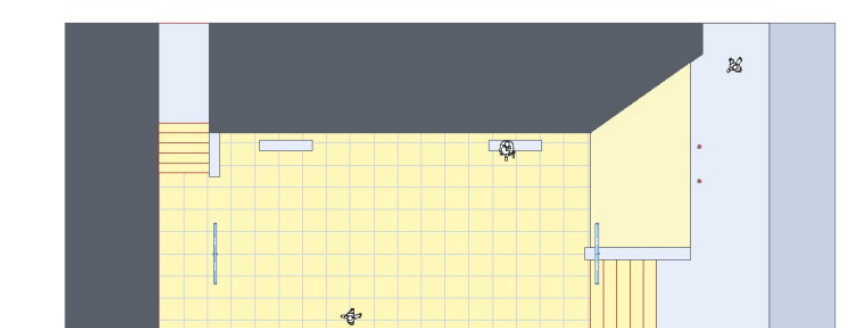

Safety (CPTED)

- Social safety

Varies between daytime and nighttime, and with the level of attractive

ness the square/court-

yard has towards people

as a destination. And also

with the type of building

uses of the surrounding

buildings overlooking the

site.

- Evening Lighting

A barrier is created if

a clear route is not lit

through the square.

- Spatial design

Design features and

obstacles can create hid-

den areas, as well as few

entry and exit points of-

iso, if to

loc, courtham

main stret, and sur-

rounding buildings have

no people present aft

can become completely

isolated.

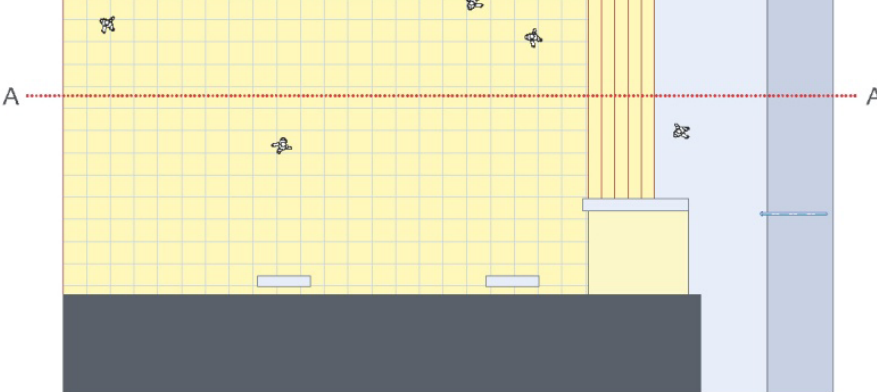

Plan
Proximity \& legibility

Route continuity

Can become a barrier

conditions, as the

conditions, as the ped

exposed.

- Perception of Proximity

More direct routes can

be achieved as space

allow access through the

building fabric.

\section{Legibility}

re

map, and often serve as

map, and often serve as

significance to the com

munity.

Path quality \& comfort

Connectivity (Macro)

Footpath quality

Characteristics of

paved. - Density

NA

Comfort

weather conditions, par-

weather condions, par-

trian only space with the

tran only space with

nance. Also less noise

is present due to the

location of the square/

courtyard often being set

back from the road.

Shelter from weather

No shelter is provided

square/courtyard.

Building land use NA barriers

Reduces block sizes

\section{Linkages}

Being large paved urban

informal spaces, square

courtyard can squar

and increase the diver-

sity of spaces within th

pedestrian network.
High, under desirable

when passing across the

Treatment of significant

$$
\text { pletely }
$$

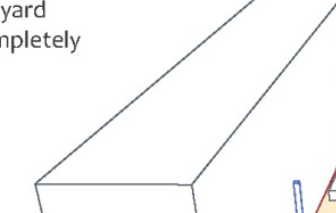

Interest

- Engagement with surrounding context

Physically high, as users are given free regn to teract within the setting.

\section{- Social interest}

Is weather permitting but can be very high if the setting is a popular leisure place, well used thoroughare, or if buildings interact with the square/courtyard. Also the informality of the space often permits the space often permits culture and community activity.

- Built interest

Varies with the variety of landscaping, art, archisurrounding perimeter buildings.

\section{- Natural scenery}

Dependant on the degree of planting/landscaping. 
Thoroughfare- Surrogate

Description

- A thoroughfare that is created by passing through privately

owned land such as a school or housing complex.

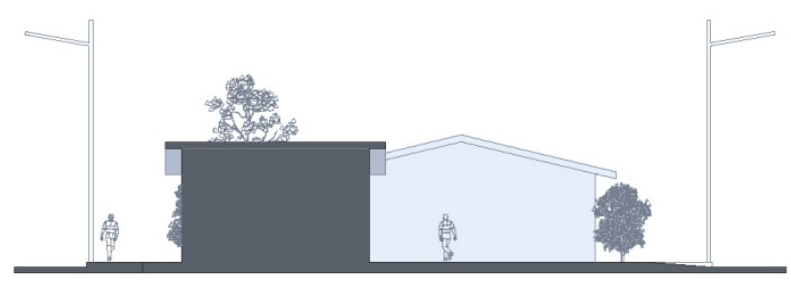

Section AA

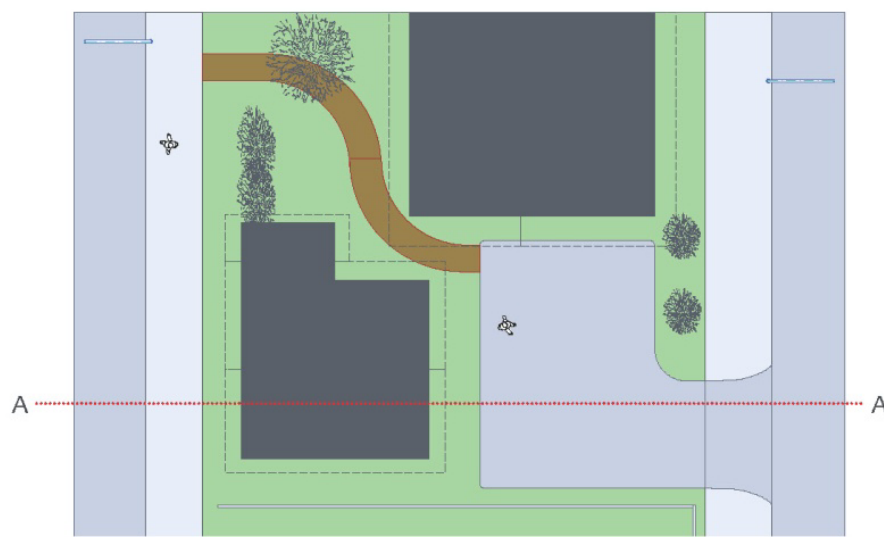

Plan
Proximity \& legibility

- Route continuity

Is reduced greatly in the

evening due to the $a b$ -

sence of people around

when the bulde cases

land is occupied by the

tenants, such as a schoo

where it is inappropriate

to trespass during schoo

- Route proximity

Improves journey prox-

imity by allowing direct

thoroughare between

ing fineness to the street

network thus reducing

block sizes.

- Legibility

Routes are local and not

located on a map or are

signposted.
Safety (CPTED)

- Social safety

Is a barrier when the

ver for the day,

as no people are around.

- Evening Lighting

the private tenant.

- Spatial design

is required to navigate

between and around

buildings/obstacles, the

spatial layout creates

opportunities for alcoves.

Thus the user can be-

come hidden from the

main street and thus dur-

inin street and thus dur-

becomes dangerous.

Characteristics of the

- Footpath quality

Quality relies on the pri-

vate tenant.

- Building land use

Shelter from weather

s dependant on whether

ave covered

walkways throughout the

site. This is only useful if

the tenants are comfort-

-Treatment of significan

Reduces block sizes.

Connectedness be

mprove the linkages

between streets in the

ble with members of the

public using their facili-

\section{Comfort}

Users may feel out of

place and uncomfortable

interaction with the land

tenants.

Noise/air quality

Improves as the user

moves away from the road.

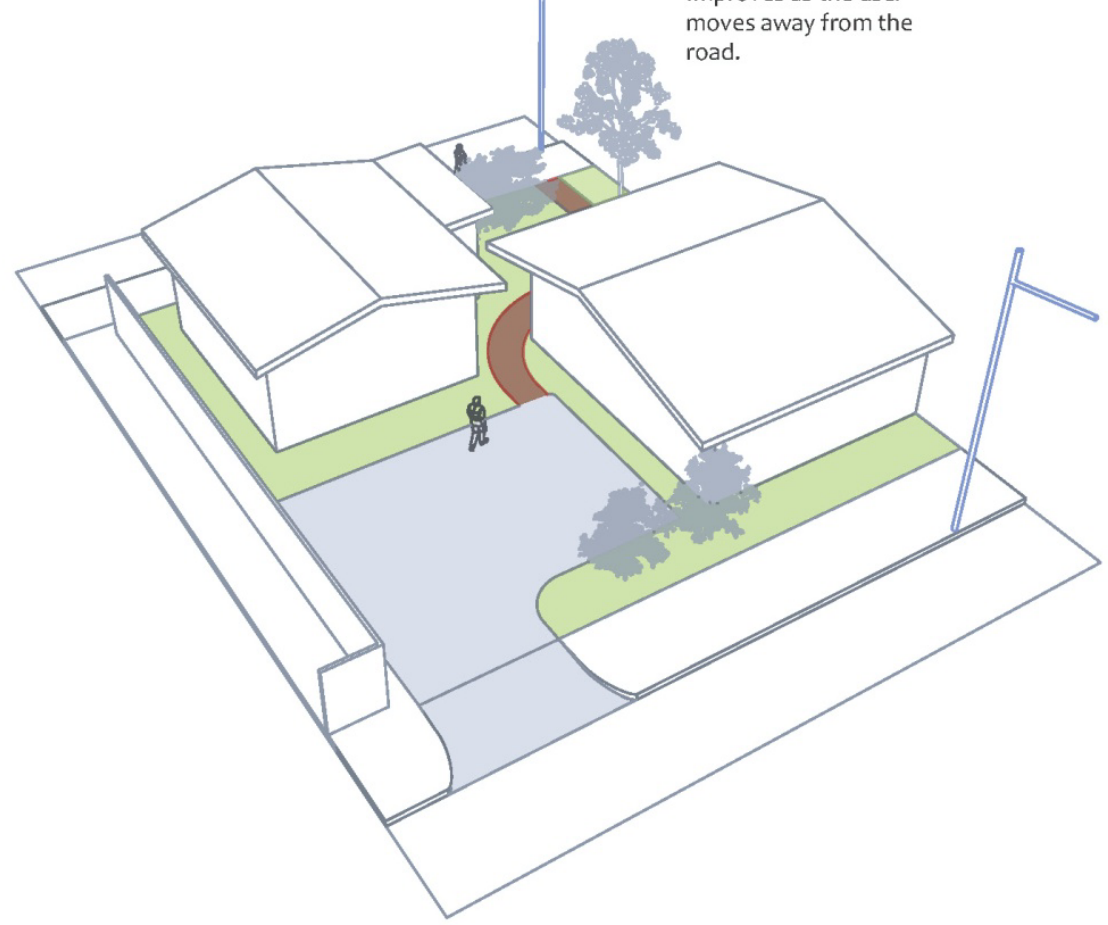

- Engagement with surrounding context

Engagement is limited if

users is restricted to the

edge of property to avoid

interaction with building

tenants. However after

hours users can navigate

freely through the site.

- Social interest

None due to absence of

social activity.

- Visual interest

route through site allows

interaction with the ten-

Natural scenery

Varies between sites. 
(Land use typologies) 


\section{Street - Heavy Industrial}

\section{Description}

A street permitting pedestrian and vehicle use, but is overtly vehicular orientated due to the high volume of industrial related activity such as couriering / storage of goods. Characterised by large sections and warehouse type buildings that hold mass storage of goods.
- Route continuity After hours the street can

become unused when

the buildings are absent

of evening activity, and

road vehicles use is low.

Also disruption in poor

weather as there is no

shelter.

- Route proximity

The big scale of the large

sections, roads, and typi-

cally warehouse build-

ings, are less appropriate

for the pedestrian scale

users. Psychologically this

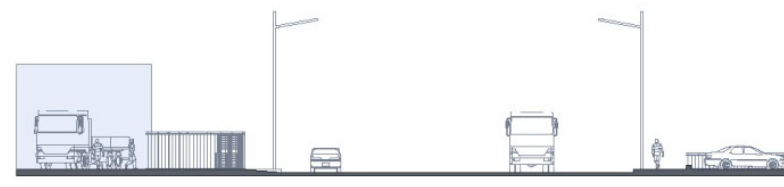

slower.

- Legibility

Section AA
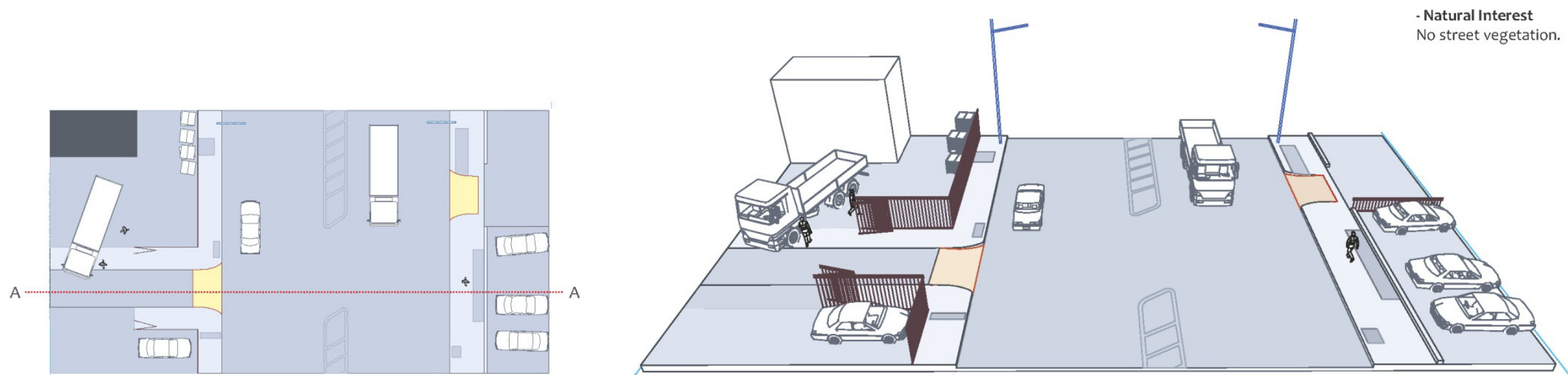

Path quality \& comfort

- Social safety

All activity is absent after

hours in the surround-

ing buildings, thus socia

.

- Evening Lighting

Only street lighting afte

hours.

- Spatial design

The large lots, which are

broken up by fences and

building structures, cre-

many secluded areas

off the footpath edge at

night. Also, the footpath

by vehicle access ramps

that are dangerous to $p$

destrians when vehicles

exit and enter properties.

- Path quality

maintained causing the

surface to be patchy and

uneven. Also users are

terrupted frequently by

vehicle access ways.

- Comfort

If the street is part of a

significant arterial road,

noise from trucks and

heavy road use, as well

comfort is also reduced

due to the lack of ameni-

ties such as vegetation,

paving

Shelter from weather

None as no canopies ar

present, and buildings

are setback far from the footpath.
Connectivity (Macro)

- Characteristics of the

land use pattern:

The land sections

required for the in-

is problemattic as it

creates less walkably

enviornments. Large

single use building and

sections means there

is fewer variety of land

use taking place within

a walk able area. This

results in increased

travel distances to

alternative land uses.

Building land use

-Treatment of significant barriers

- Linkages

NA
Interest

Engagement with the surrounding context

Deep building set backs,

parking lots separating

building, and typically

ew building openings to

the street, limit physical

and social interaction

buildings.

\section{Social interest}

Relies on buildings having

openings that are visible

ple a workshop.

Built interest

Buildings often have

large blank facades,

which can be perceive

as being dull or boring.

However some interes

could be offered if mer-

chandise is stored at the

front of the section.

Natural Interest 


\section{Vegetation-Bank/Gully}

\section{Description}

- Dense vegetation that runs adjacent to the footpath. It can

be further distinguished by being on either a bank or gully.
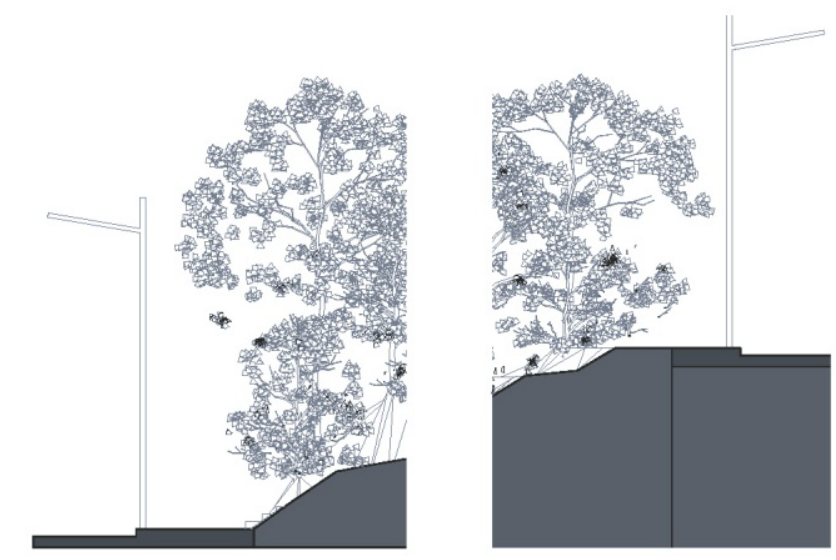

Section Bank

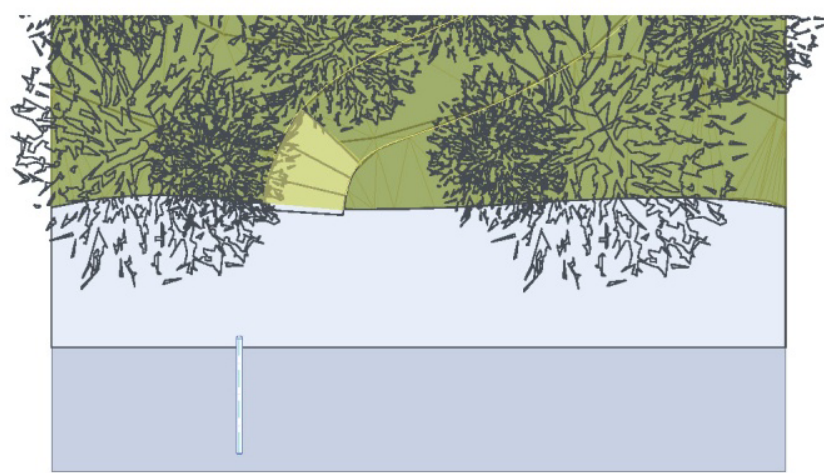

Plan

\section{Section Gully}

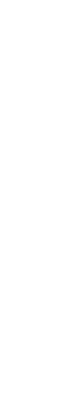

(1)
Proximity \& legibility

- Route continuity

A range of CPTED prob-

lems can cause walking

past vegetation in the

nighttime to become a

barrier.

- Route Proximity

- Perception of Proximity Some types of vegetation can cause a high level of interest and therefore can reduce the perception of distances.

\section{- Legibility}

\section{NA}

Social safety

Evening Lighting

Large vegetation dra

matically darkens the

footpath which is danger-

ous for two reasons: The

lack of visibility of the

footpath surface in terms

of pedestrians potentially

visibility into ane

tion where people could

linger.

- Spatial design

Dense foliage creates

hidden areas off the foot-

path where people could

linger.

Footpath quality

Poorly maintained veg.

etation can encroach

onto the footpath which

is problematic because

it can: Reduce the width

of the footpath; cause

potential trip hazardous;

fllen leaves can become

slippery when wet.

\section{Comfort}

The presence of the

vegetation contributes to

a sensuously pleasing set

ting, and also improves

air quality.

-Shelter from weather

Large vegetation in the

form of natural canopies

can offer shelter from the

rain in places, and dense

vegetation shields the

wind.

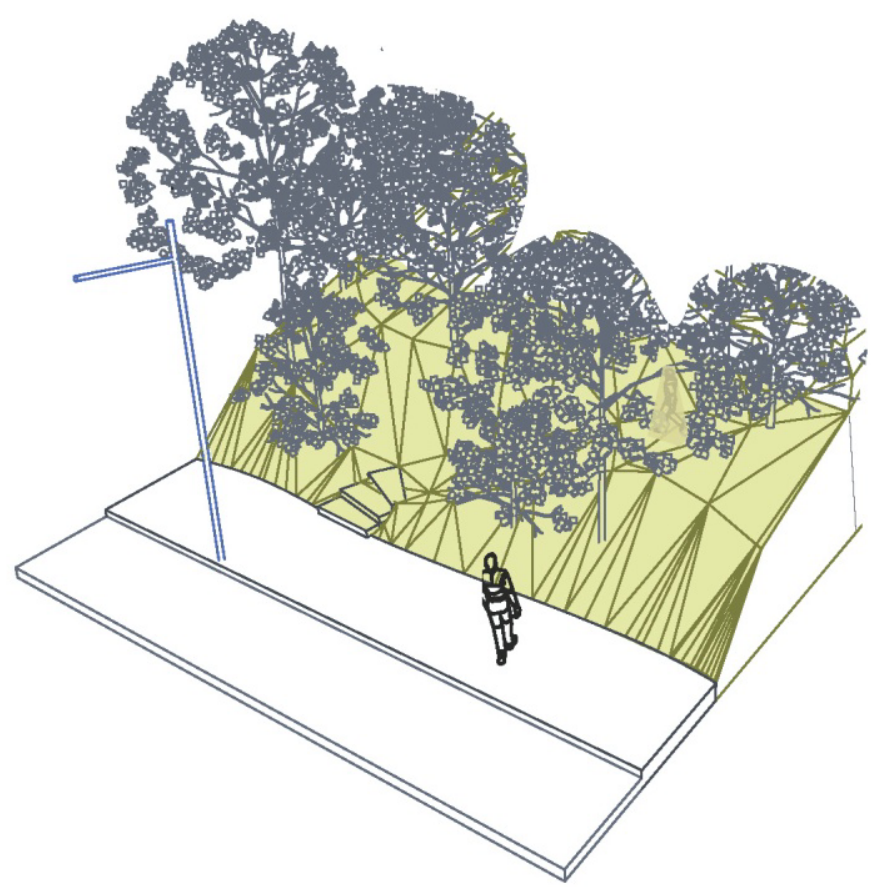

Interest

Enectivity

Characteristics of the

- Treatment of significan

pedestrian barriers

Significant vegetation

fect of extreme weathe

Engagement with surrounding context

Engagement with the

natural surroundings de-

pends on how close the

ation encroaches to

ever dense vegetation

Connectedness be-

tween paths within the

network

- Built interest

NA

Natural scenery

High as users are im-

mersed in the natural

vegetation surroundings. 
Street - Retail/Hospitality

Description

A pedestrian and vehicle street characterised by having retail/ hospitality stores at ground level. Typically there is a high presence of pedestrian amenities and roadside parking to access the stores.

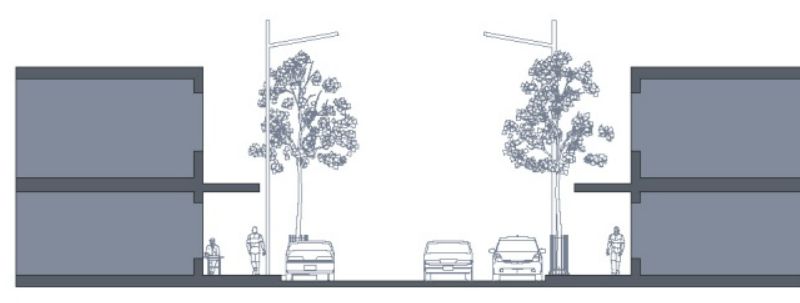

Section AA

Proximity \& legibility

Path quality \& comfort

Connectivity (Macro)

Interest

- Social safety - Route continuity

In the case of retail stores

Afterhours the street can

become unused if there

social activities at ground

floor dissipate after

is an absence of building

uses that proved evening

of passing vehicles, thus activity.

'eyes on the street' is

crucial for maintaining

sense of safety. This is re-

liant on either, Mixed Use

\section{- Proximity}

More direct routes can

More direct routes can

the most direct route

dis, ENTE activiss being users may trave further

present at ground floor

to

present

presence of social activity

ping street offers This

ping street offers. This

particularly after hours if

the street offers evening

activity and a sense of so-

cial safety. Also this high

level of interest reduce

journey perceptions of

walking lengths.

Footpath quality

- Characteristics of the

land use pattern:

Density

Comfort

There is a greater presence of amenities such

as rubbish bins, seating,

vegetation etc.

- Shelter from weather

High due to the presence of canopies and veran-

- Safety from vehicles

Relies on buffering from

on street parking, plant

ing, and traffic calming

measures to reduce the

speed/noise from passing

ground floor window

displays, and underside

randa.

- Legibility

NA
NA

Building land use

Walkability and social

safety improves if build

ings are mixed use. This

is because people can

live closer to needed

shops, and also provide eye's on the street due

to the residential on

the floors above.

Treatment of significant barriers

Linkages

NA
- Engagement with surrounding context Physically, the width of the path limits its informath talking etc, particularly talking etc, particularly under high use. Socially ghen stores spill out when stores spillout and

\section{- Social interest}

Social activity is high during opening hours due to the localization of consumer activities attracting people to the stree whilst transparency of ground floor windows permitting views into stores users.

\section{- Built interest}

Window displays, store interior fit outs, and architectural appeal of the surroundings buildings offer visual interest.

- Natural scenery Dependant on the extent of street planting.
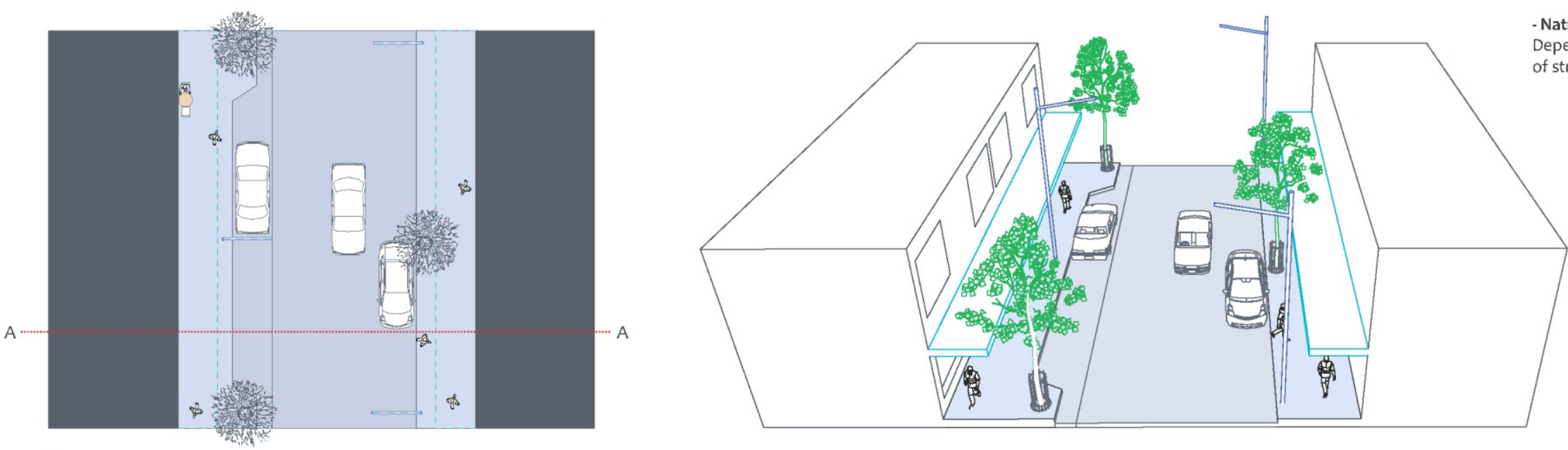

Plan 


\section{Back Street - Commercial}

\section{Description}

A pedestrian and vehicle street characterised by having sec-

ondary frontages of commercial/office type land use. Because

of their location away from, or at the rear of, the main hospital-

ity and retail frontages, Commercial Back Streets have fewer

pedestrian amenities and activity open to the ground floor

than Shopping Streets.

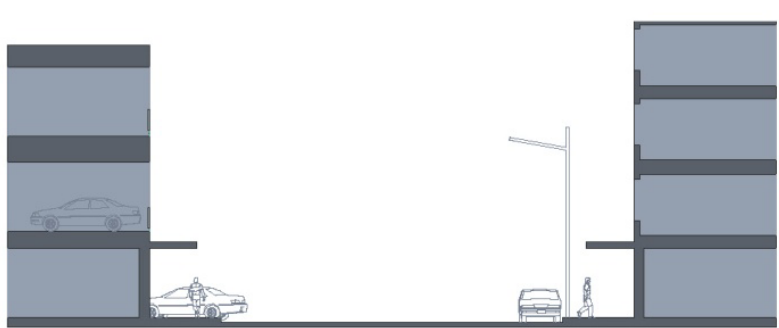

Section AA
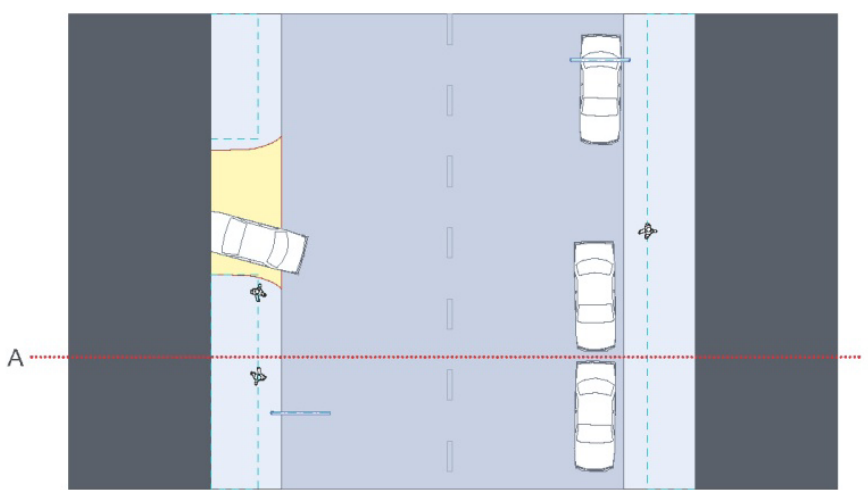

- Social safety

In the case of commer-

cial/office building use,

unless there are hospi-

floor, social safety is only

in the form of passing

vehicles.

\section{- Evening Lighting}

A combination of street

lighting, and where

ground floor retail is

present, the secondary

lighting from window

\section{displays.}

- Spatial design

Vehicles using back en-

trance ramps to building

and car parks are haz-

ardous for pedestrians.

Also blank ground floor
facades, and absence of

residential apartments,

remove possibilities of

ENTE (evening night tim economy) activities and thus eyes on the street.

Route continuity

After hours the absence

of building activity at

ground floor can become

a safety barrier to pedes

Perception of proximity When blank ground floor footpath edge, i.e. at the rear of the site or in with interest can psychologcally lengthen walking

distances for pedestrian

- Legibility

\section{Buffering from vehicles}

requent car parking

entrances are dangerous

- Shetrefrom weather

Shelter from weather

Relies on canopies

present on the sides and

aniding facades.

Noise and Air quality

Significant vegetation,

amenities, and interest,

is needed to counter the

poorer quality pedestrian

environment due to hig

presence of vehicles. walls are present at the building uses such as car parking buildings. Lack of

and disruptive to pedes

- Characteristics of the

and use pattern:

NA

- Building land use

NA

- Treatment of significant

barriers

- Linkages

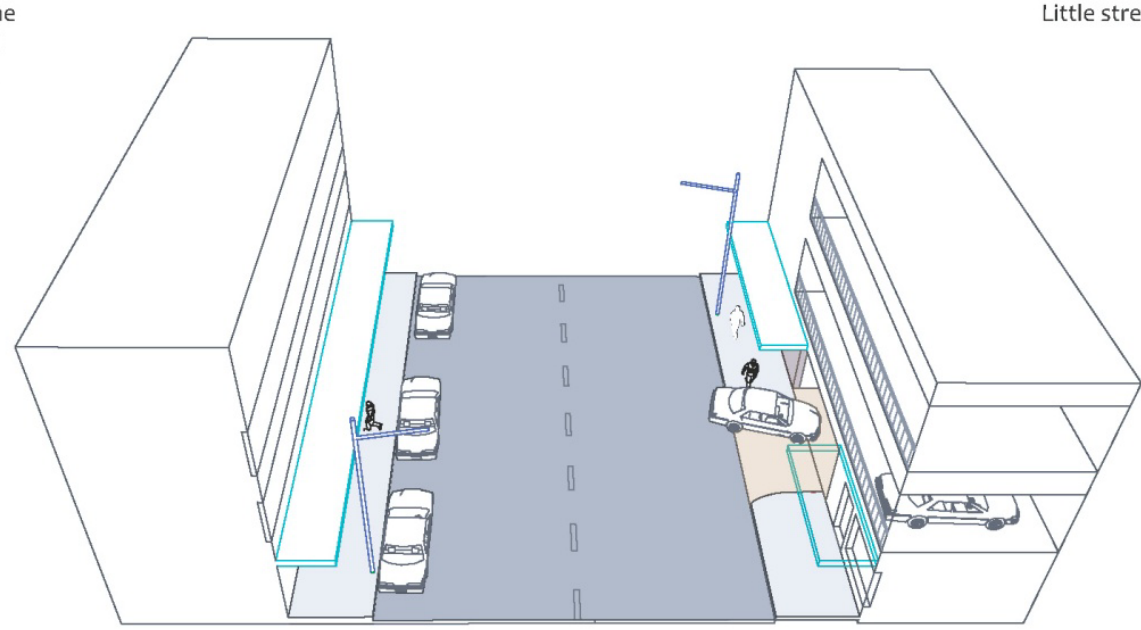

Plan 
Street-Residential Suburban

Description

A street permitting pedestrian and vehicle use. Land use characterized by having low density residential housing properties set back on generous open sections. Pedestrians are separated from vehicles due to wide footpaths and street planting/grass verges.
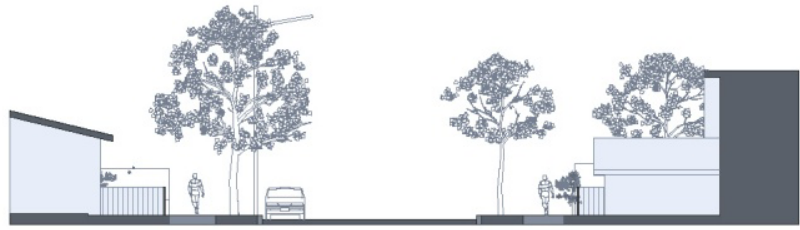

- Route continuity

Atthough people are pre sent in the houses after ie. users feeling i.e. users feeling they ar not visible whilst on the footpath can become walking barriers to pedestrians. Another barrier also being the absence shelter from weather.

- Perception of proximity Tall and blank boundan fences that block views into properties, as well as wide sections, can psychologically lengthe walking distances for pedestrians.

\section{- Legibility} N/A

Section AA

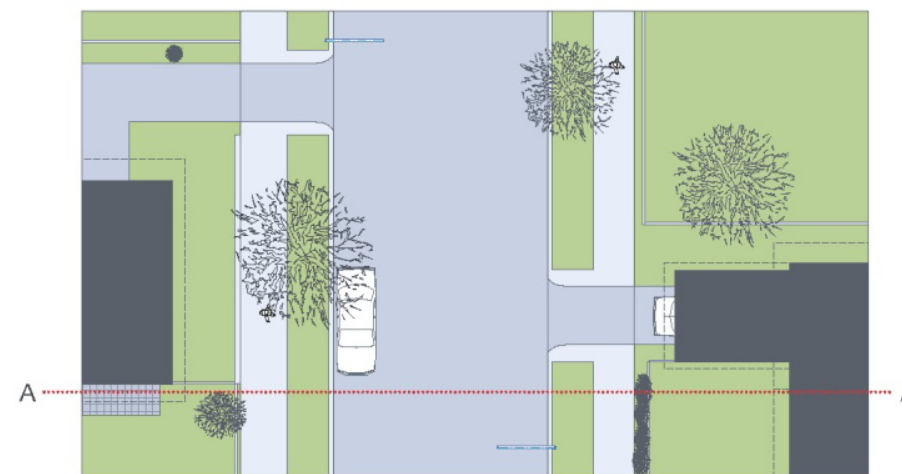

A

Plan
- Social safety

Residential social activity ispresentafter hoursin

\section{- Evening Lighting}

Street lighting only after

hours, however large

trees can block light from

the street lamps thus creating dark and concealed areas.

\section{- Spatial design}

'Eyes on the street' can

be reduced significantly if

houses are setback deep

in to sections, tall fences/

hedges and dense veg-

etation block views into

properties, and footpath

lighting is inadequate.

Also regarding safety

from passing vehicles,

vehicle buffer to the road users.

quality

Buffering from vehicles Street trees and grass give a sense of safety an eparation away from passing cars.

\section{Shelter from weathe} No shelter from the weather, unless street trees are dense and frequently planted.

\section{- Noise and Air quality} Unless on along side a well used vehicle thoroughfare, the greater presence of vegetation and wider footpaths buffering from the road, offers pleasant noise and air quality.

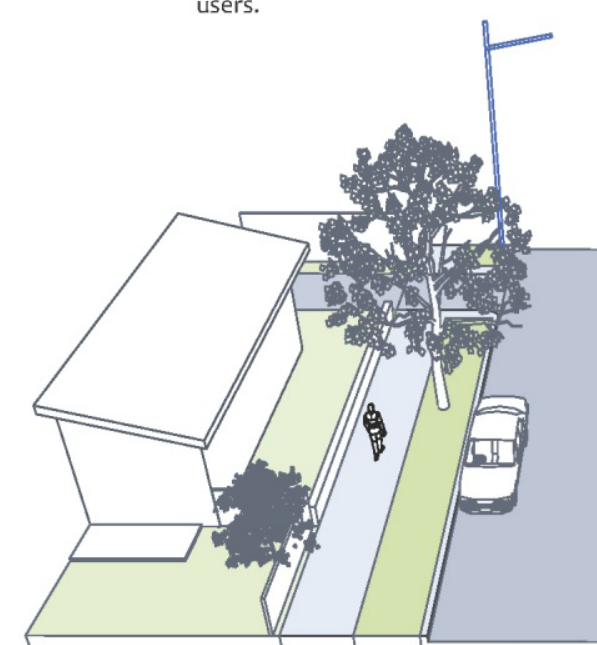

- Characteristics of the and use pattern

- Density and building

Walkable connections between a variety of and uses, relies on the disbursement of town centers within residential suburbs existing more frequently. Thus inlow density subur ban developments, town centers require larger catchment areas in order to meet their demands. Which intern increases walking trip distances.

-Treatment of significan barriers

NA

- Linkages

NA
Engagement with the surrounding context obstacles such as high property fences and deep bulding set backs, prevent interaction between pedestrians and neighboring properties. - Social interest Relies on buildings having openings that are visible from the street exposing internal activity for exan ple a workshop.

Social interest

Relies on buildings and properties having openings that are visible from the street thus exposing residential activity.

- Built interest Interest offered through the variety of housing and garden types.

Natural Interest verges, vegetation from properties, and where permitted views into property gardens. 


\section{Street - Light Industrial}

Description

A street permitting pedestrian and vehicle use, but is vehicle orientated due to the high volume of couriering and vehicle related activity that accompanies light industrial activity i.e. auto repair stores. Sites are charcterised by having much smaller sections than heavy industrial sites such as garage and single unit/store workshops at ground level.

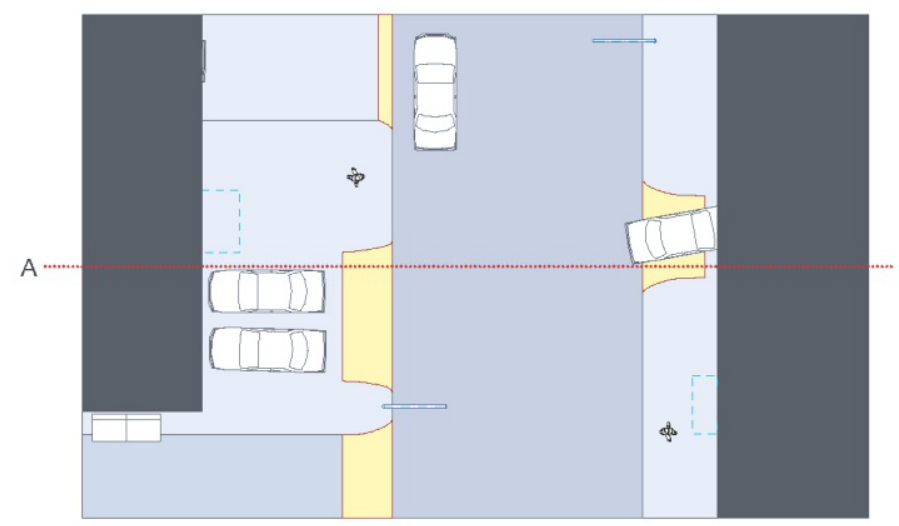

A

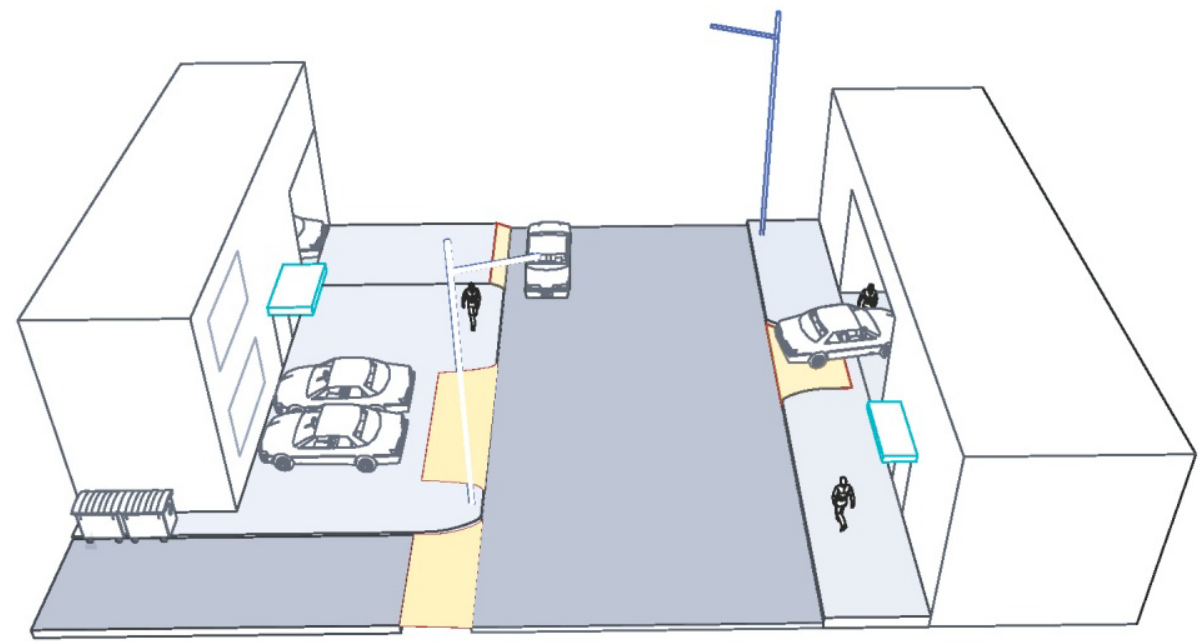

Proximity \& legibility

Path quality \& comfort

Connectivity (Macro)

Interest

- Social safety

- Route continuity

become unused when

of evening activity, and

road vehicles use is low.

Also users receive

users.

Evening Lighting

eet lighting only after hours.

\section{Spatial design}

as front parking lots, can

create places for people

to linger after hours. Also

the footpath is frequen

interrupted by vehicle

access that is danger-

ous to pedestrians when

properties.

\section{- Proximity}

If buildings are significantly set back from the footpath edge, psychologically this causes walking to feel slower.

\section{- Legibility}

Footpath quality

Users are interrupted fre

quently by vehicle access

Comfort

Pedestrian comfort is re

duced due to the lack of

amenities such as vegeta

tion, rubbish bins, quality

paving.

-Shelter from weather

No canopies present.

- Safety from vehicles

The high presence of

vehicles on or around the

ant.

- Characteristics of the

land use pattern:

Density

Building land use

Because industrial is

considered an undesir-

able land use to be near

residential develop-

ments, when in large

clusters they can create

voids of residential land

use. This can contribute

ble environment.

-Treatment of significant barriers

NA

- Linkages

NA
Afterhours the street can

Bullding setbacks such

vehicles exit and enter

- Engagement with surrounding context

Physically, is limited by

building setbacks and

parking lots separating

the footpath users from

the buildins. Whist so-

cial engagement limited

by whe location

of building openings, an

the building setbacks,

tween the footpath use

and building user.

- Social interest

Relies on buildings expos-

ing internal activity, for

example workshop acti

ity, which can be seen

from the street.

- Built interest

Window displays, store

interior fit outs, and ar-

chitectural appeal of the

surroundings buildings

offer visual interest.

- Built interest

Interest is offered

through views into the

building exposing the as-

sociated tools/merchan-

dise/storage etc, of the

space requirements of

the building tenant.

No street vegetation 


\section{Mall - External}

Description

A pedestrian only street with consumer related tenancy at

ground floor level.

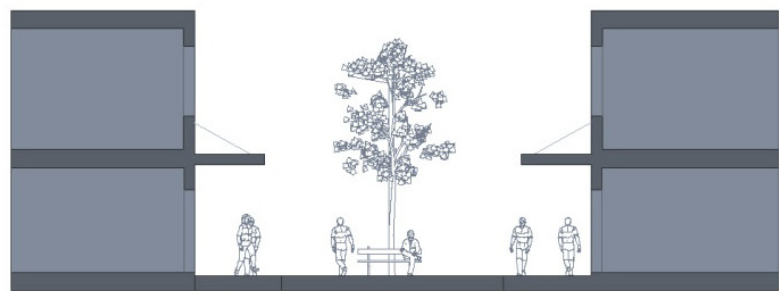

Section AA
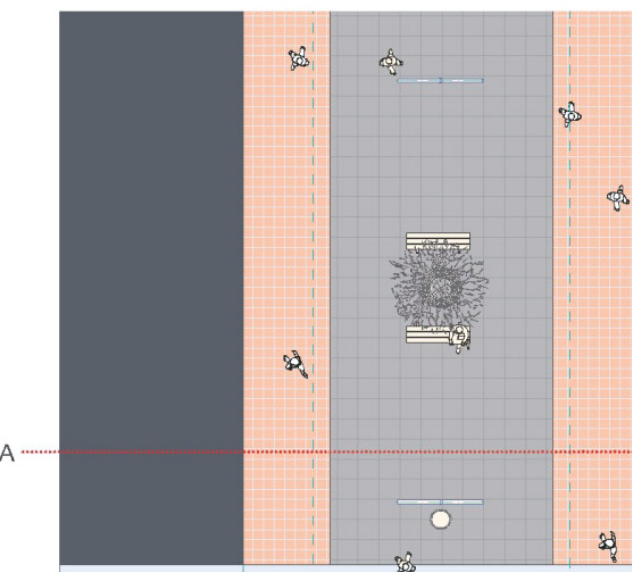

Plan

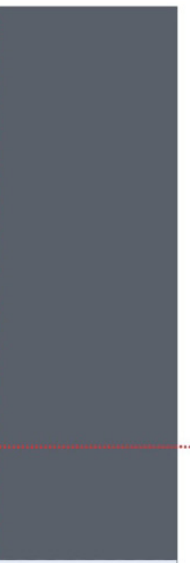

- Social safety

In the case of retail

stores, social activities

at ground floor dissipat

after hours thus 'eyes on

the street' is crucial for

maintaining a sense of

safety. This is reliant on

either, Mixed Use de-

velopments, residential,
or late night hospitality

activities being present at

ground floor and also th

above floor levels.

- Evening Lighting

bination of pole street

lighting, secondary light-

ing from ground floo

underside lighting and

the veranda.

-Spatial design

NA

cur after hours when the

street becomes unused.

This is due to the com-

bination of an absence

the reduction of ground

floor activity when the

stores close. This is less

of a problem if the build

ings are mixed use with

the above stories being

residential.

- Perception of Proximity

Groundfloor spaces off

a high level of interest

which can redces the

users perception of the

length of their journey.

\section{- Legibility}

NA

Footpath quality

Comfort

quality pedestrian en-

vironment. Users are

there is a high presence

of interest from store

during opening hours;

the street is quieter be-

ing away from vehicular

traffic.

- Shelter from weather

Relies on building cano-
Interest

Characteristics of the land use pattern:

Mall complexes/

clusters of shops can

ecome problematic.

As they draw shop ten-

ants from other town

centres throughout

ing to some shops not

Building land use

Social safety, and

walkabiliy improves if

shops are mixed use

with residential above.

- Treatment of significant

barriers

NA

Linkages

Engagement with sur-

rounding context

Physically, because the

space is pedestrian only,

this allows users the

freedom to navigate and

occupy the space as they

please. Socially a high

level of interaction can

exist when stores spill

out and occupy the street

space.

- Social interest

ittle unless the path is frequently used, or offers

que views into/over

- Built interest

Subject to the design of the over bridge and sur-

Natural scenery

Is dependent on whether views are offered to the

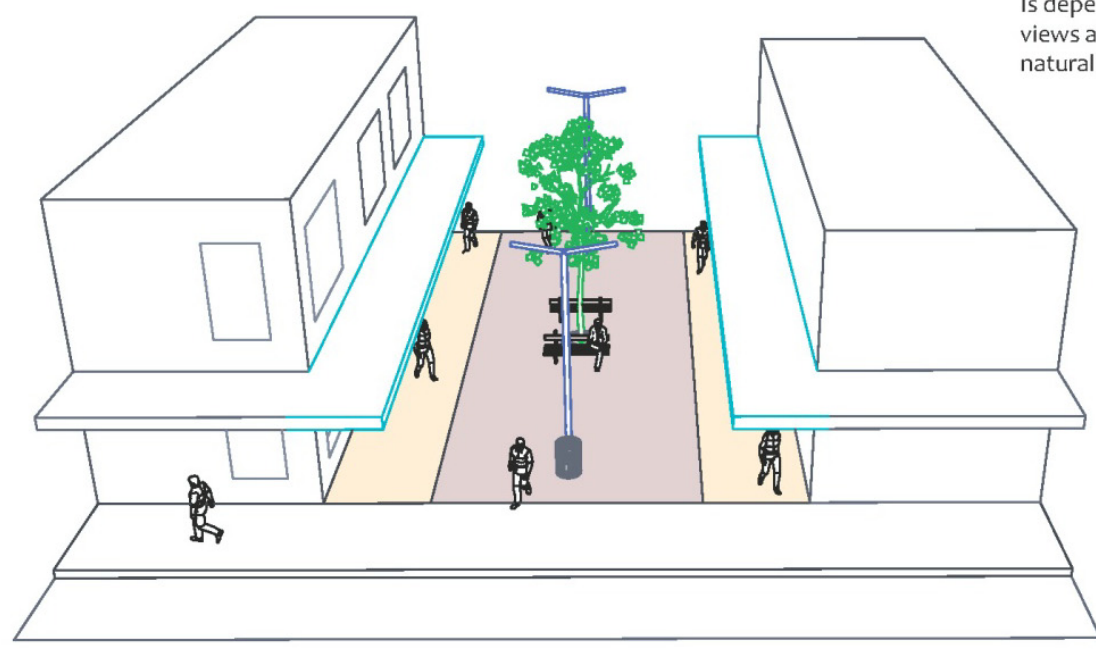




\section{Appendix (B): Cognitive survey pilot test}

(Pilot test) Walking in Central Wellington

Interviewers Question Schedule

1)

Locate on the map of Central Wellington:

- The location of residency within $30 \mathrm{~m}$ approximately.

- The most distant location you would feel comfortable walking to on a daily basis.

- Maximum comfortable duration you would walk?

- Two necessary activity destinations you would normally drive to, but you think are of a 'walkable' distance?

- Why do you drive to these destinations as appose to walk?

2)

During any weather condition, time of day, or under any circumstance, would you not walk to any of the destinations above? (i.e. rain, in the dark, heavy vehicle traffic, crowding, after hours, Saturday nights due to presence of drunk people etc)

What routes would you take during the daytime under calm weather if you had to walk rather than drive to the destinations you stated in question 2 ?

- Think about the route you chose, as best you can describe:

- Why you choose to walk down certain streets over others?

- Where you crossed the roads you encountered:

How do you cross each road? i.e. J walk, or use the crossing

- Which places to cross are most desirable and why?

- Which places to cross are least desirable and why?

If you had to walk rather than drive to the destinations in question 2 , draw on the map the route or routes you would take during nighttime under calm weather

- Think about the route you chose, as best you can describe:

- Why you choose to walk down certain streets over others?

Where you crossed the roads you encountered?

- How do you cross each road? i.e. J walk, or use the crossing

- Which places to cross are most desirable and why?

- Which places to cross are least desirable and why?
If you had to walk rather than drive to the destinations in question 2, draw on the map draw the route you would take during the daytime under extreme weather conditions.

- Think about the route you chose, as best you can describe:

- Why you choose to walk down certain streets over others?

Where you crossed the roads you encountered:

- How do you cross each road? i.e. J walk, or use the crossing

- Which places to cross are most desirable and why?

- Which places to cross are least desirable and why?

If you had to walk rather than drive to the destinations in question 2, draw on the map draw the route you would take during the nighttime under extreme weather conditions.

- Think about the route you chose, as best you can describe:

Why you choose to walk down certain streets over others?

- Which streets are most desirable and why?

Which streets are least desirable and why?

Where you crossed the roads you encountered:

How do you cross each road? i.e. J walk, or use the crossing

- Which places to cross are most desirable and why?

Which places to cross are least desirable and why? 
Appendix (C): Cognitive survey 1 questionnaire 


\section{THO VICTORIA

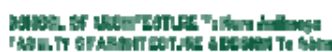 \\ Treas war J

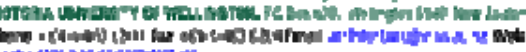

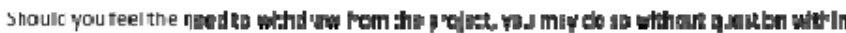

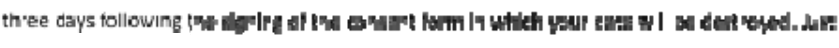
suniast me Michatlel town

\section{Use and storage of thiformotion}

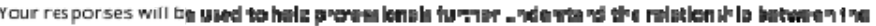

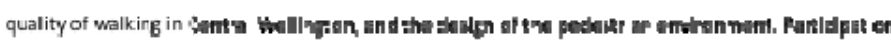

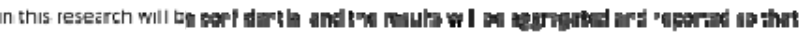
participates are nen-tie retladip.

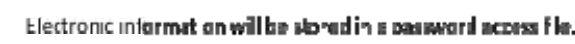

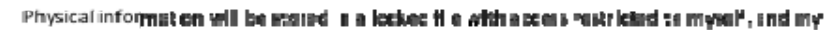

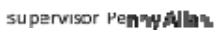

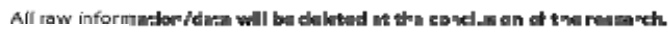

If you have any questlons abors. the prdect please cottac: ne.

\section{Michael Lowe}

ph: 0273587985

enail: cnanneI__@

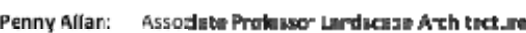

ph: 044639460

email: penty.allan@unvas $\mathrm{m}$

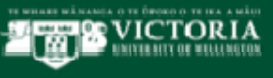

Ris

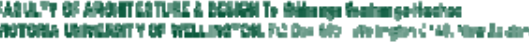

\section{Informatlen shees}

Research Project:

The 'Walking for Drtving' project

Investigator: Misha al Lowe

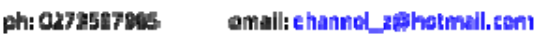

Supervisor: Penny Allan

ph: OA 403 me0

anall: panny. alan

Participant infermnt|sn shees for a ssudy of Walking Perceptlons

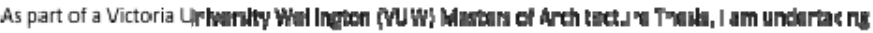

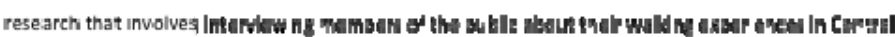

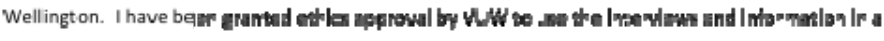

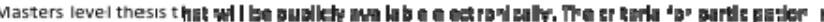

- Live in central Wa lir

- Drive in Central We Ir

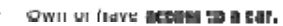

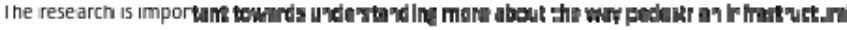

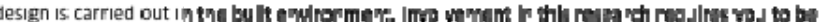

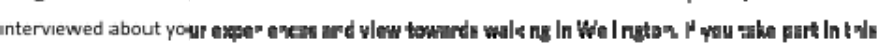

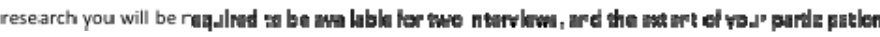
would be:

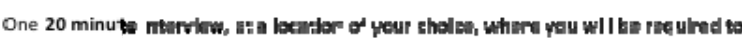

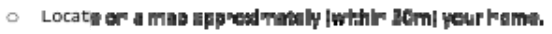

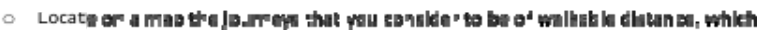

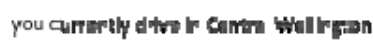

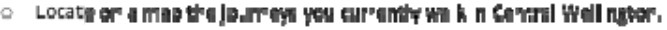

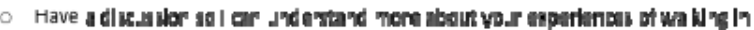
wellingran.

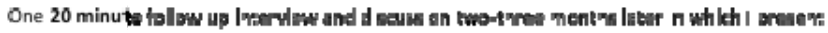

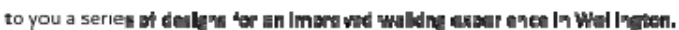




\section{Iiv VICTORIA}

Whot

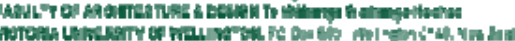

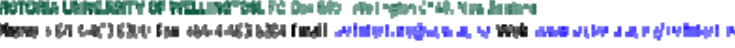

\section{Consent Form}

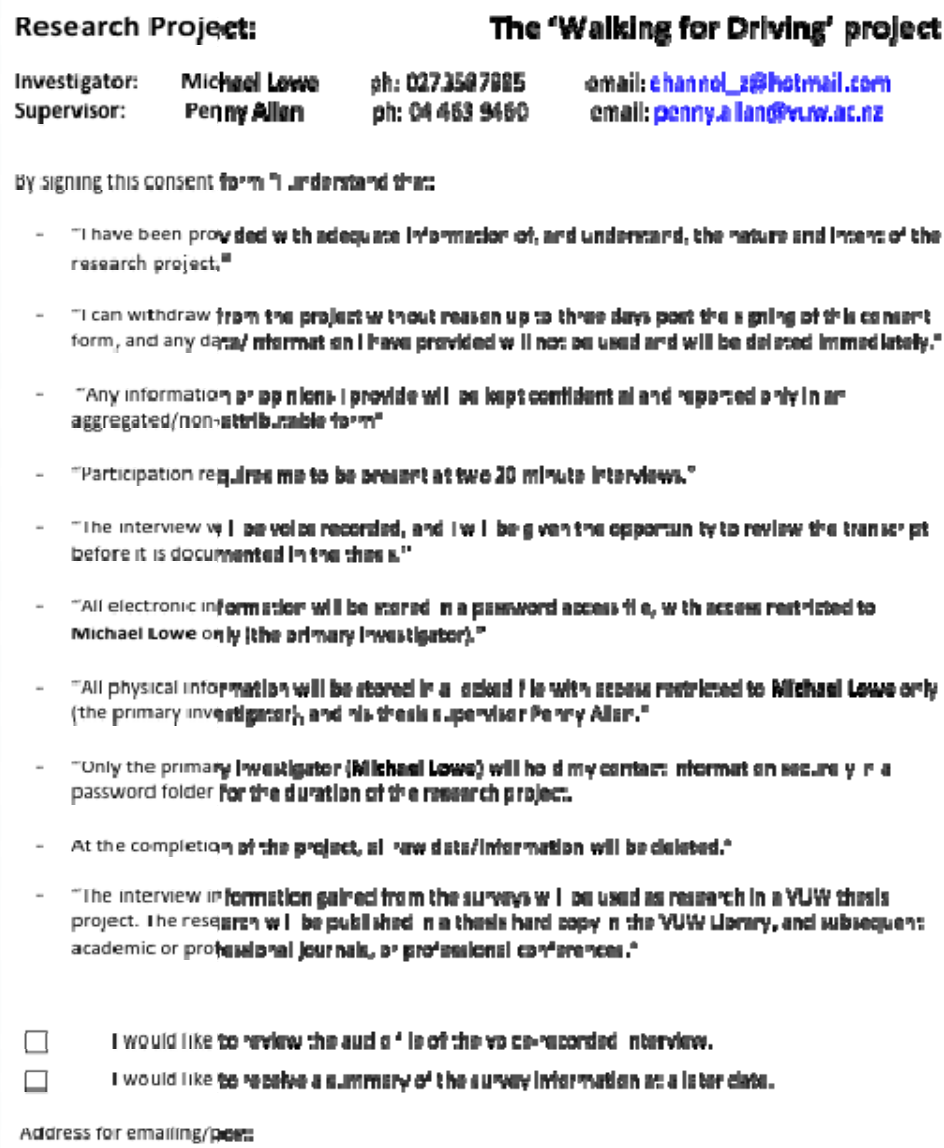

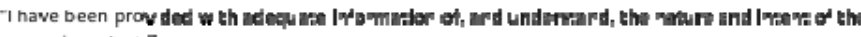
rasearch project."

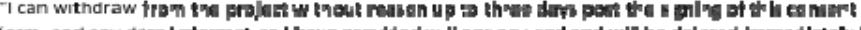

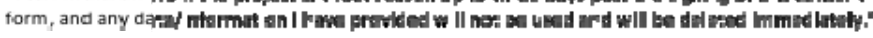

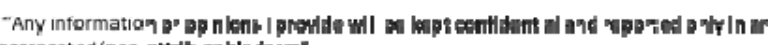
ageregated/nonn

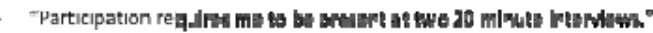

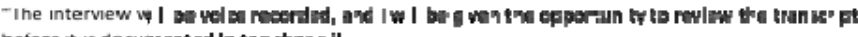

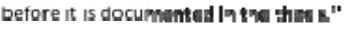

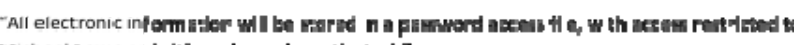

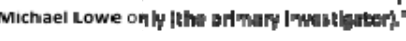

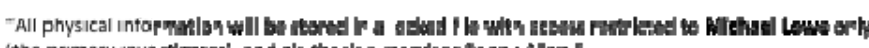

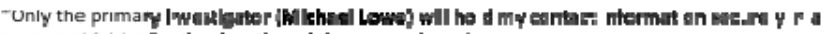

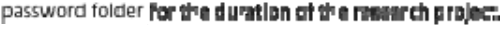

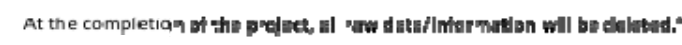

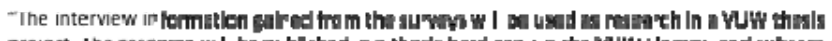

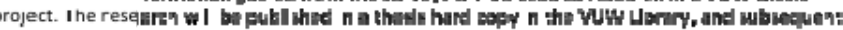

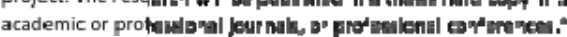

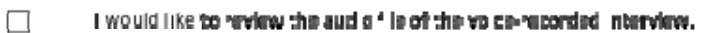

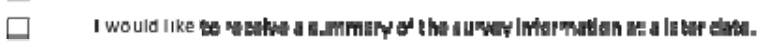

Address for emalling/pera

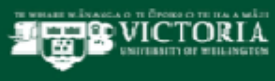

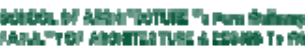

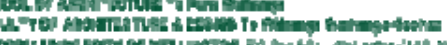

w

\section{Interylewers Question Schedule}

Research Project

The 'Walking for Driving' project

\section{onail! ehanthe aghotnaileg}

Penmôlin phe

1) Generally, when werforming a neersary adwity on a fite day:

a. What $k$ tha manmum combrortabla Altitanco pou would walk for kafore pou dacidad you would retrer throt?

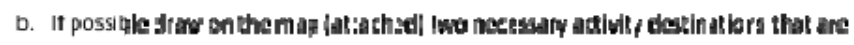

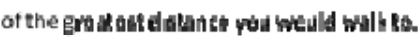

2) For a necessary adivity, locate in tie area allocites on the nap (atteched) the dest tnations

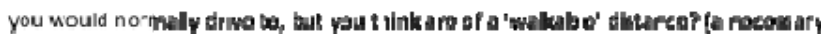

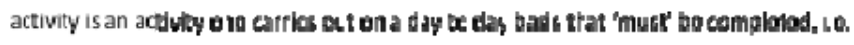

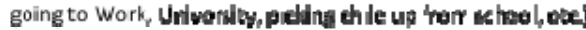

3) Are there reasqns why pot drive to thoce diatiationa as oppose bo walt

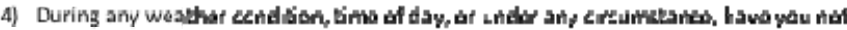
walked to any of tie destiations sbove? fic. nan, in the derk, heroy wehicke trafic. crowding, after heurs, Saturtay nights due to trunt worth etej

5) Hypothetically / pau had to walk pather than dino to tro dast nedions you babed in question 2, draw en tha map the routger nutos you would tak an a unny dey a. Think a hat the route pou chose.

i. As toat you can ouplain why pou choose bo walk down ectlan stroobs over others? 


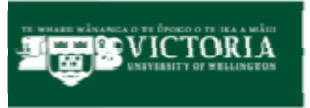

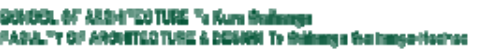

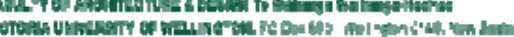

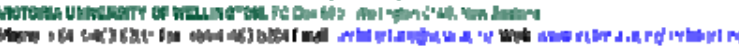

II. At bask vou can, anplan where you creak tha read and how l.a.d walk before the liahte?

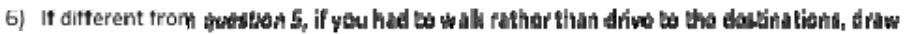

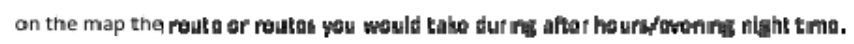

a. Think ateut the reube pou tinera,

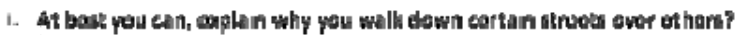

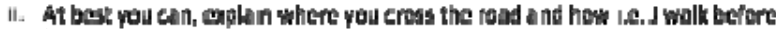
tha listiti?

7) If difterent trom puestion 4 and 5, if yeu hat to wall rathar than drive to the deatinatiens, draw on the map draw the reute you would than during mitrame want har condhent.

a. Think atyout the rouls pou chese,

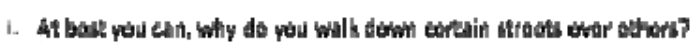

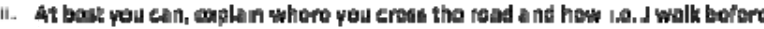
the lightt?

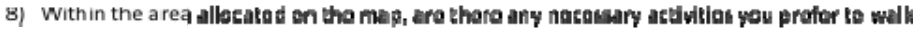
to instead or driva?

a. Locate the reutex on the map lattached|

b. Why don't yeu dr rwe to thave leertions?

c. What enticos you to wall to tha co dostination

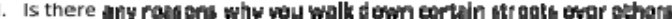

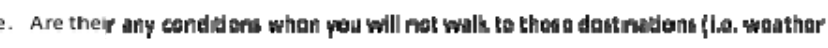
condition, time of diay, $\mathrm{Qtex}$ 
Appendix (D): Wellington road hierarchy (obtained from Wellington City Council 2012) 


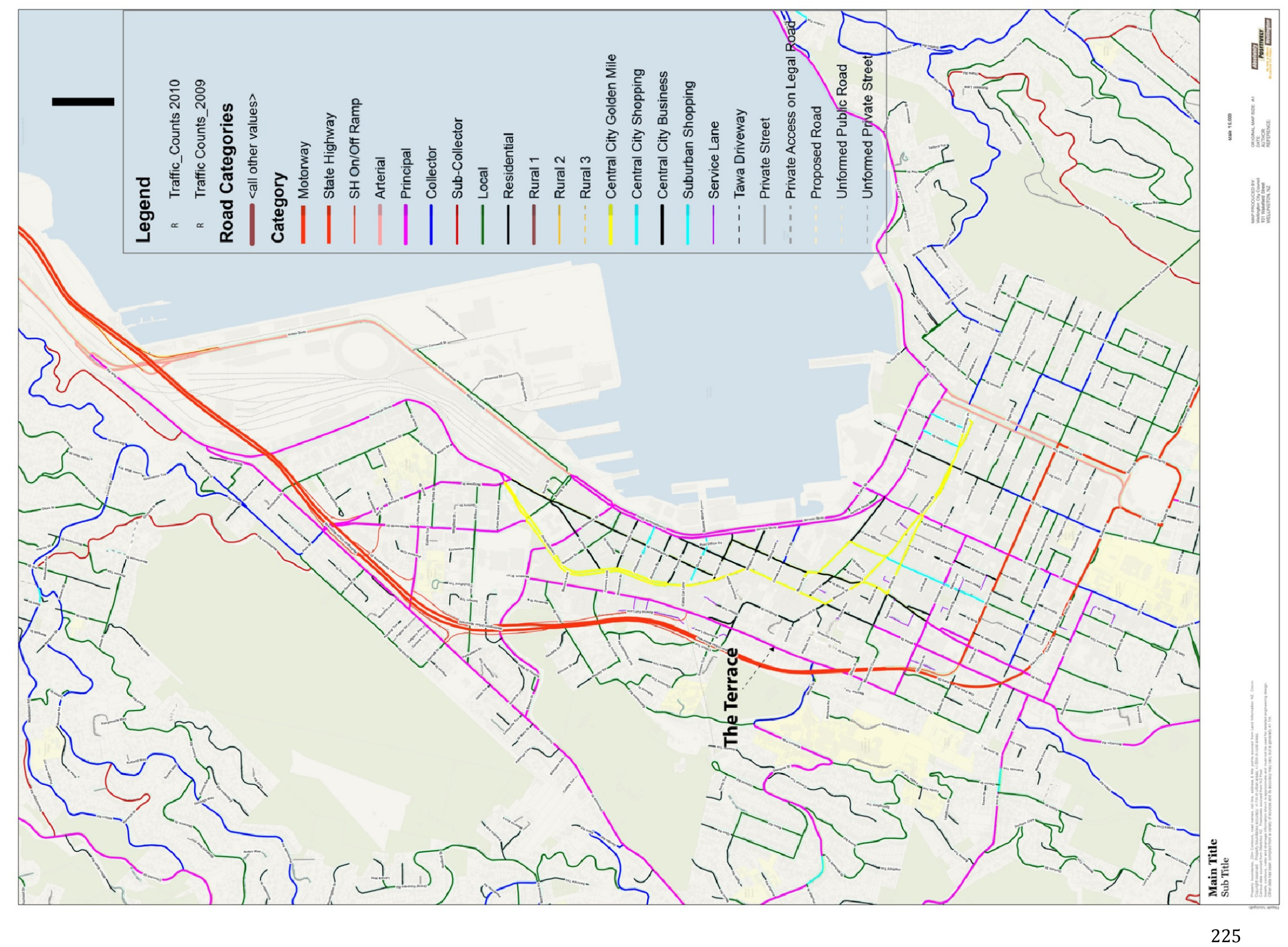


Appendix (E): Table review of pedestrian design practise in Central Wellington 


\begin{tabular}{|c|c|c|c|c|c|c|c|c|c|c|c|c|c|c|c|c|c|c|c|c|c|c|c|c|c|}
\hline \multirow[b]{3}{*}{$\begin{array}{c}\text { Policy } \\
\text { Document }\end{array}$} & \multirow[b]{3}{*}{$\begin{array}{l}\text { Element } \\
\text { category }\end{array}$} & \multirow[b]{3}{*}{ Built element } & \multirow{3}{*}{ 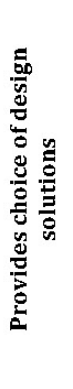 } & \multirow{3}{*}{ 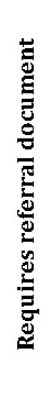 } & \multicolumn{21}{|c|}{ Pedestrian Accessibility Criteria } \\
\hline & & & & & \multicolumn{5}{|c|}{ Safety } & \multicolumn{3}{|c|}{$\begin{array}{c}\text { (Macro scale) } \\
\text { Proximity and } \\
\text { Legibility }\end{array}$} & \multicolumn{7}{|c|}{ Path Quality and comfort } & \multicolumn{3}{|c|}{$\begin{array}{l}\text { (Macro scale) } \\
\text { Connectivity }\end{array}$} & \multicolumn{3}{|c|}{ Interest } \\
\hline & & & & & 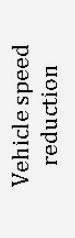 & 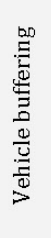 & 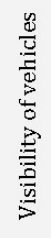 & 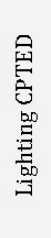 & 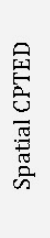 & 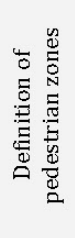 & 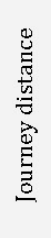 & 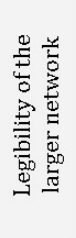 & 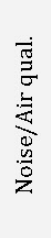 & 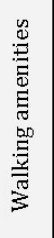 & 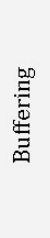 & 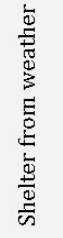 & $\begin{array}{l}\stackrel{3}{=} \\
\frac{\pi}{4} \\
\frac{\pi}{2} \\
\sum\end{array}$ & 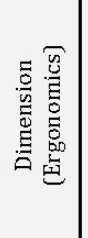 & 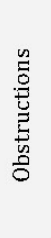 & 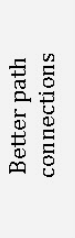 & 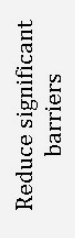 & 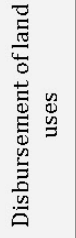 & 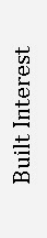 & 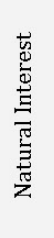 & 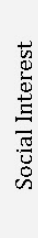 \\
\hline \multirow{13}{*}{$\begin{array}{c}\text { Pedestrian } \\
\text { Planning } \\
\text { and Design } \\
\text { Guide }\end{array}$} & \multirow{5}{*}{ 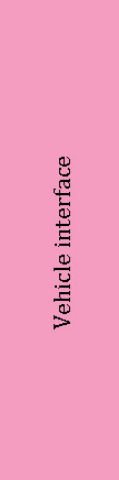 } & $\begin{array}{l}\text { Road (traffic } \\
\text { calming) }\end{array}$ & & 1. & $\sqrt{ }$ & & & & \multirow{13}{*}{ 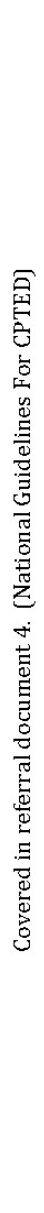 } & & & & $\sqrt{ }$ & & & & & & & & & & & & \\
\hline & & Intersections & Yes & & $\mathbf{R}$ & & $\mathbf{R}$ & & & $\sqrt{ }$ & & & & & & & & $\sqrt{ }$ & & & & & & & \\
\hline & & $\begin{array}{c}\text { Crossings \& } \\
\text { platforms }\end{array}$ & Yes & & $\sqrt{ }$ & $\sqrt{ }$ & $\sqrt{ }$ & & & $\sqrt{ }$ & & & & & $\mathbf{R}$ & & & $\sqrt{ }$ & & & & & $\mathbf{R}$ & & \\
\hline & & $\begin{array}{l}\text { Zebra \& } \\
\text { pushbutton } \\
\text { crossings }\end{array}$ & & & $\sqrt{ }$ & & $\sqrt{ }$ & & & & & & & & & & & $\sqrt{ }$ & & & & & $\sqrt{ }$ & & \\
\hline & & Driveways & & & $\sqrt{ }$ & & $\sqrt{ }$ & & & $\sqrt{ }$ & & & & & & & & $\sqrt{ }$ & & & & & & & \\
\hline & \multirow{8}{*}{$\begin{array}{l}\text { Pedestr } \\
\text { ian } \\
\text { access }\end{array}$} & Pedestrian precinct & & & & $\mathbf{R}$ & & & & & & & $\mathbf{R}$ & & & & & & & & & & $\mathbf{R}$ & & $\mathbf{R}$ \\
\hline & & Shared zone & & & $\mathbf{R}$ & $\mathbf{R}$ & & & & & & & & $\mathbf{R}$ & & & & & & & & & $\mathbf{R}$ & & $\mathbf{R}$ \\
\hline & & Shared main street & & 4 & & $\mathbf{R}$ & $\mathbf{R}$ & & & & & & $\mathbf{R}$ & & & & & & & & & & & & \\
\hline & & Footpath & Yes & & $\mathbf{R}$ & & & & & $\mathbf{R}$ & & & & & $\sqrt{ }$ & $\mathbf{R}$ & $\sqrt{ }$ & $\sqrt{ }$ & & & & & $\mathbf{R}$ & & \\
\hline & & $\begin{array}{l}\text { Off street } \\
\text { pedestrian } \\
\text { network }\end{array}$ & & & & & & & & & $\mathbf{R}$ & & & & & & & & & & & & & & \\
\hline & & Shared path & & 2. & & & & & & $\sqrt{ }$ & & & & & $\mathbf{R}$ & & & $\sqrt{ }$ & $\mathbf{R}$ & & & & & & \\
\hline & & Grade separation & & & NA & $\sqrt{ }$ & NA & $\mathbf{R}$ & & & $\sqrt{ }$ & & & & & & & $\sqrt{ }$ & & & $\mathbf{R}$ & & & & \\
\hline & & Ramps and steps & Yes & & & & & & & & & & & $\sqrt{ }$ & & & $\sqrt{ }$ & $\sqrt{ }$ & & & & & & & \\
\hline
\end{tabular}




\begin{tabular}{|c|c|c|c|c|c|c|c|c|c|c|c|c|c|c|c|c|c|c|c|c|c|c|c|}
\hline & & $\begin{array}{l}\text { Public transport } \\
\text { shelter }\end{array}$ & & & & & & & $\mathbf{R}$ & & & & & & $\mathbf{R}$ & $\sqrt{ }$ & $\sqrt{ }$ & $\mathbf{R}$ & & & & & \\
\hline & 窇 & Street furniture & Yes & & & $\mathbf{R}$ & & & & & & & & $\sqrt{ }$ & & $\sqrt{ }$ & $\sqrt{ }$ & $\sqrt{ }$ & & & $\mathbf{R}$ & & \\
\hline & & Path Landscaping & & & $\mathbf{R}$ & & & & & & & & $\mathbf{R}$ & $\sqrt{ }$ & & $\sqrt{ }$ & & & & & $\mathbf{R}$ & & $\mathbf{R}$ \\
\hline & & Lighting & & 3. & & & $\mathbf{R}$ & $\mathbf{R}$ & & & & & & & & & $\mathbf{R}$ & $\mathbf{R}$ & & & & & \\
\hline \multirow{8}{*}{$\begin{array}{c}\text { District } \\
\text { plan }\end{array}$} & \multirow{8}{*}{ 岕 } & $\begin{array}{l}\text { Connections to the } \\
\text { city }\end{array}$ & & & & & & & & & & & & & & & $\mathbf{R}$ & & $\mathbf{R}$ & & & & \\
\hline & & $\begin{array}{l}\text { Moving through } \\
\text { the city }\end{array}$ & & & & & & & & $\mathbf{R}$ & & & & & & & $\mathbf{R}$ & & $\mathbf{R}$ & & & & \\
\hline & & $\begin{array}{l}\text { Routes through } \\
\text { buildings }\end{array}$ & & & & & & & & & & & & & & & & & & & $\mathbf{R}$ & & $\mathbf{R}$ \\
\hline & & Way finding & & & & & & & $\mathbf{R}$ & $\mathbf{R}$ & & & & & & & & & & & & & \\
\hline & & Public space & & & & & & & & & & & & & $\mathbf{R}$ & & & & $\sqrt{ }$ & & $\mathbf{R}$ & & $\mathbf{R}$ \\
\hline & & Streets & & & & & & & & & & & & & & & & & $\mathbf{R}$ & & $\mathbf{R}$ & & \\
\hline & & Squares & & & & & & & $\sqrt{ }$ & & & & $\mathbf{R}$ & & & & $\sqrt{ }$ & & & & $\mathbf{R}$ & & $\mathbf{R}$ \\
\hline & & Parks & & & & & & & & & & & & & & & & & & & $\mathbf{R}$ & & $\mathbf{R}$ \\
\hline \multirow{3}{*}{$\begin{array}{c}\text { People } \\
\text { Spaces } \\
\text { Places : A } \\
\text { Design } \\
\text { guide for } \\
\text { Urban NZ }\end{array}$} & \multirow{3}{*}{$\stackrel{\Xi}{0}$} & Urban structure & & & & & & & $\mathbf{R}$ & & $\mathbf{R}$ & & & & & & & & $\mathbf{R}$ & $\mathbf{R}$ & $\mathbf{R}$ & $\mathbf{R}$ & \\
\hline & & $\begin{array}{l}\text { Development } \\
\text { zones }\end{array}$ & & & & & & & $\mathbf{R}$ & & & $\mathbf{R}$ & & & & & & & & $\mathbf{R}$ & $\mathbf{R}$ & & $\mathbf{R}$ \\
\hline & & Streets & & & & $\mathbf{R}$ & & & & & & & & & $\mathbf{R}$ & $\mathbf{R}$ & & & & & $\mathbf{R}$ & & \\
\hline
\end{tabular}

$\sqrt{ }=$ The document comprehensively demonstrates an awareness of the accessibility criteria

$\mathrm{R}=$ The document only states an awareness of the accessibility criteria. 


\section{List of referral documents}

1

- Guide to traffic engineering practice, part 10: Local area traffic management (Austroads)

- $\quad$ Sharing the main street and Cities for tomorrow: better practice guide, (Hans L part C-5)

2.

- $\quad$ Australian Bicycle Council: Pedestrian- cyclist conflict minimisation on shared paths and footpaths (ARRB Group)

3.

- $\quad$ AS/NZS 1158.3.1: 1999, Road Lighting - Pedestrian area (Category P) lighting - Performance and installation design requirements (Joint Technical Committe LG/2 Road Lighting)

4.

- National Guidelines for Crime Prevention Through Environmental Design. (Ministy of Justice) 
Appendix (F): Cognitive survey 2 questioner (front and back) 


\section{Information Sheet}

59 EVICTORIA Research Project:

Investigator: Michael Lowe

Supervisor:

The 'Walking for Driving' project

ph: 0273587985

ph: 044639460

email: channel_z@hotmail.com

email: penny.allan@vuw.ac.nz

\section{Participant information sheet for a study of Walking Perceptions}

As part of a Victoria University Wellington (VUW) Masters of Architecture Thesis, I am undertaking research that involves interviewing members of the public about their walking experiences in Central Wellington. I have been granted ethics approval by VUW to use the interviews and information in a Masters level thesis that will be publicly available electronically. The criteria for participation are:

$$
\text { - Live in Central Wellington and are of age between 18-25 years. }
$$

The research is important towards understanding more about the way pedestrian infrastructural design is carried out in the built environment. Involvement in this research requires you to be interviewed about your experiences and view towards walking in Wellington.

Use and storage of information Central Wellington, and the design of the pedestrian environment. Participation in this research will be confidential and the results will be aggregated and reported so that participates are non-identifiable:

Electronic information will be stored in a password access file.

All rawa If you have any questions about the project please contact me.

$\begin{array}{ll}\text { Michael Lowe } & \text { Penny Allan: Associate.Professor Landscape Architecture } \\ \text { ph: } 0273587985 & \text { ph: } 044639460 \\ \text { email: channel_z@hotmail.com } & \text { email: penny.allan@vuw.ac.nz }\end{array}$

email: channel_z@hotmail.com

\section{Consent Form}

By signing this consent form "I understand that:

"I have been provided with adequate information of, and understand, the nature and intent of the research project."

"Any information or opinions I provide will be kept confidential and reported only in an aggregated/non-attributable form"

"All electronic information will be stored in a password access file, with access restricted to Michael Lowe only (the primary investigator)."

"All physical information will be stored in a locked file with access restricted to Michael Lowe only (the primary investigator), and his thesis supervisor Penny Allan."

At the completion of the project, all raw data/information will be deleted."

"The interview information gained from the surveys will be used as research in a VUW thesis project. The research will be published in a thesis hard copy in the VUW Library, and subsequent academic or professional journals, or professional conferences."

\begin{tabular}{|l|l|c|}
\hline Date & Name & Signature \\
\hline & \\
\hline
\end{tabular}

\section{$\underline{\text { Question Schedule }}$}

When you walk between Kelburn and Te Aro campus during the following time and weather circumstances below. Drawn on the attached map of VUW Kelburn campus, the route you would take through the university.

(1) Using the line style below draw your route during the daytime under calm weather conditions. . Locate on the map the places along the route you dislike the most, and explain why?

style (1) aslike - The hill going up to uns is too steep

ii. Locate on the map the places along the route you enjoy the most, and explain why? (1) like - That's the spot where I can slee the oily.

(2) Using the line style below draw your route during the daytime under extreme weather. i. Locate on the map the places along the route you dislike the most, and explain why? I (2)dislue - when I'm waiting for the bus, the wind can be pretty strong style $\&$ cold

ii. Locate on the map the places along the route you enjoy the most, and explain why?

(2) like - That's the point where I almost reach the destination 1 guess I really lice it because that the end of that extreme weather.

(3) Using the line style below draw your route during the night time under calm weather.

i. Locate on the map the places along the route you dislike the most, and explain why?

:(3) dislike - It's creepy there

style

ii. Locate on the map the places along the route you enjoy the most, and explain why? (3) like - Night usew of the city. Fantashic!

(4) Using the line style below draw your route during the night time under extreme weather. i. Locate on the map the places along the route you dislike the most, and explain why?

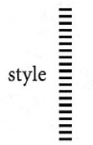

ii. Locate on the map the places along the route you enjoy the most, and explain why? 
Wähanga Waihanga-Hoaho

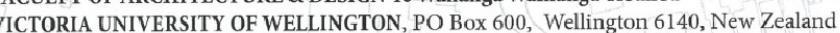

+64-4-4636200 Fax +64-4-4636204 Email architecture@vuw.acnz Web www.victoria.ac.nz/architecture

(4) 3 (2)

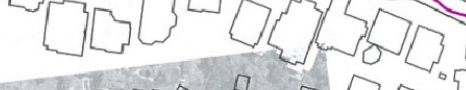

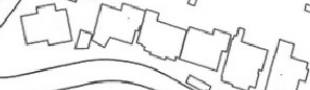
L व 5ज्ञात 5000

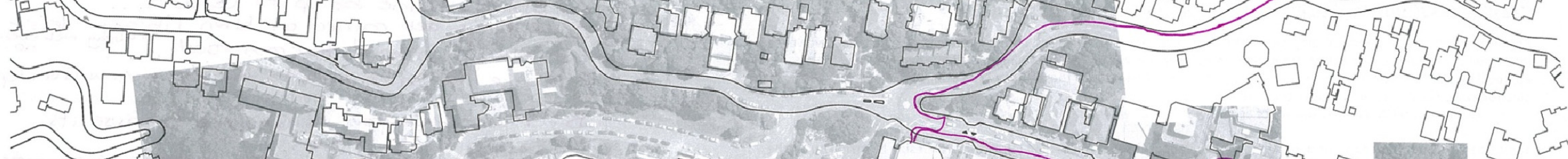

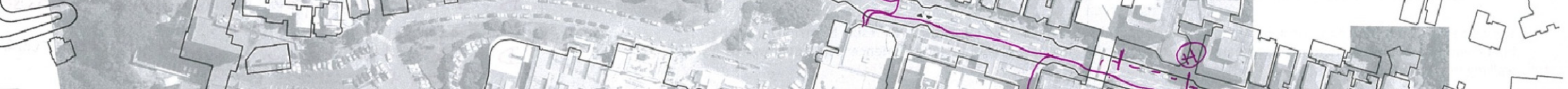
II]

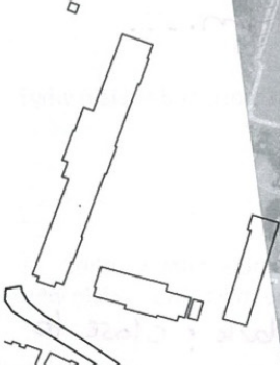

$+3-20$
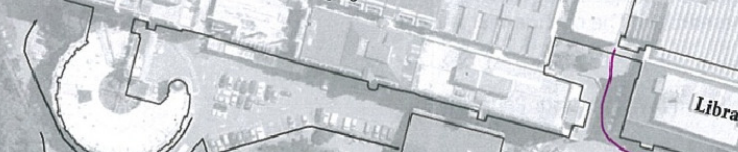

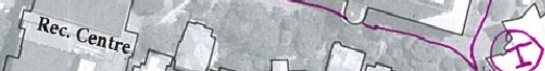

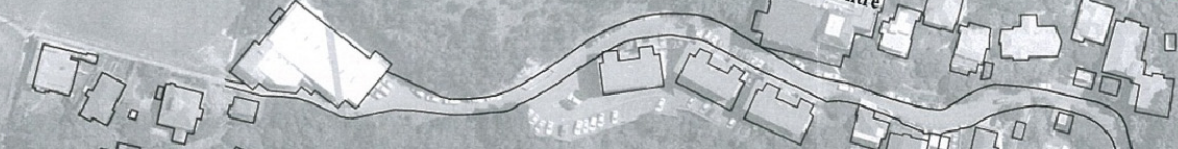

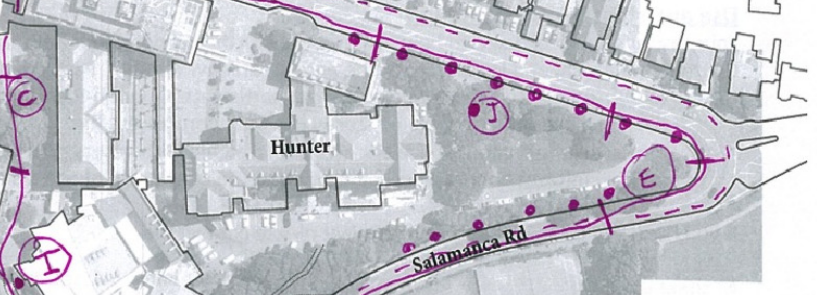

$3 x^{3}+2=27$

$\{$ और

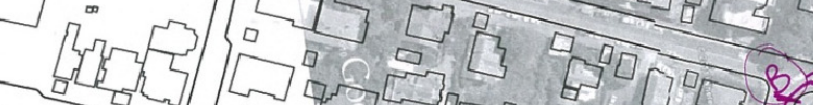

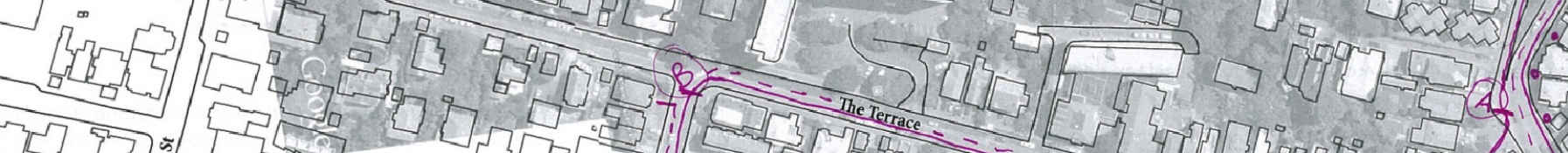

年
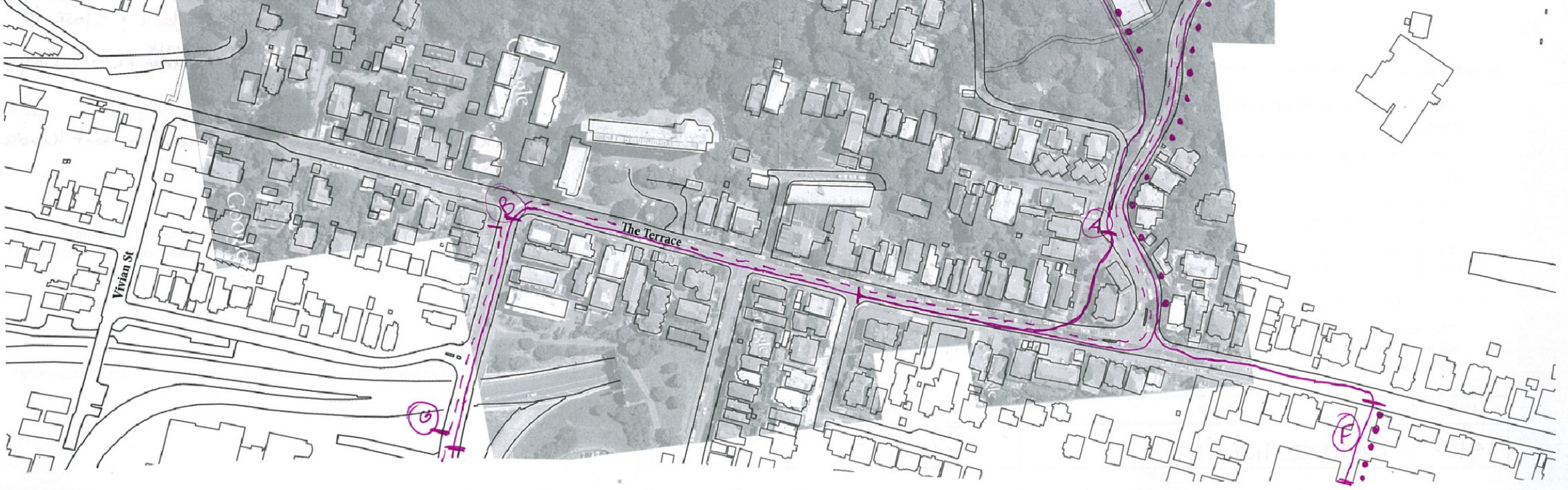
Appendix (G): Pedestrian count data (obtained from Wellington City Council 2008) 


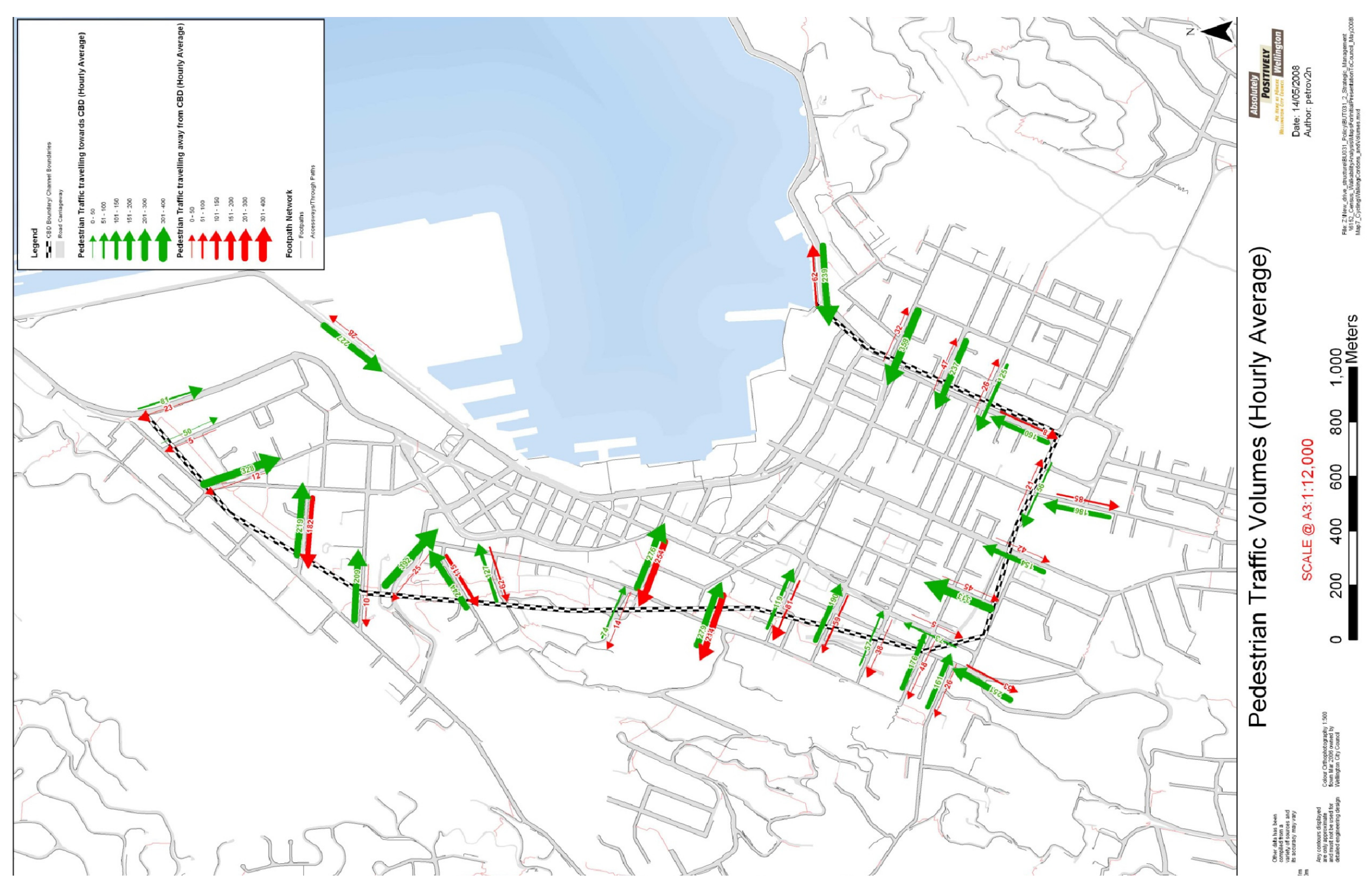

